Prepared for the U.S. Department of Energy under Contract DE-AC05-76RL01830

\title{
Techno-economic Analysis for the Conversion of Lignocellulosic Biomass to Gasoline via the Methanol-to- Gasoline (MTG) Process
}

SB Jones

Y Zhu

April 2009

Pacific Northwest

NATIONAL LABORATORY 


\title{
DISCLAIMER
}

United States Government. Neither the United States Government nor any agency thereof, nor Battelle Memorial Institute, nor any of their employees, makes any warranty, express or implied, or assumes any legal liability or responsibility for the accuracy, completeness, or usefulness of any information, apparatus, product, or process disclosed, or represents that its use would not infringe privately owned rights. Reference herein to any specific commercial product, process, or service by trade name, trademark, manufacturer, or otherwise does not necessarily constitute or imply its endorsement, recommendation, or favoring by the United States Government or any agency thereof, or Battelle Memorial Institute. The views and opinions of authors expressed herein do not necessarily state or reflect those of the United States Government or any agency thereof.

\author{
PACIFIC NORTHWEST NATIONAL LABORATORY \\ operated by \\ BATTELLE \\ for the \\ UNITED STATES DEPARTMENT OF ENERGY \\ under Contract DE-AC05-76RL01830 \\ Printed in the United States of America \\ Available to DOE and DOE contractors from the \\ Office of Scientific and Technical Information, \\ P.O. Box 62, Oak Ridge, TN 37831-0062; \\ ph: (865) 576-8401, fax: (865) 576-5728 \\ email: reports@adonis.osti.gov \\ Available to the public from the National Technical Information Service, \\ U.S. Department of Commerce, 5285 Port Royal Rd., Springfield, VA 22161 \\ ph: (800) 553-6847, fax: (703) 605-6900 \\ email: orders@ntis.fedworld.gov \\ online ordering: http://www.ntis.gov/ordering.htm
}

This document was printed on recycled paper.

$(8 / 00)$ 


\section{Techno-economic Analysis for the Conversion of Lignocellulosic Biomass to Gasoline via Methanol-to- Gasoline (MTG) Process}

SB Jones

Y Zhu

April 2009

Prepared for U.S. Department of Energy under Contract DE-AC05-76RL01830

Pacific Northwest National Laboratory

Richland, Washington 99352 


\section{Summary}

Biomass is a renewable energy resource that can be converted into liquid fuel suitable for transportation applications. As a widely available biomass form, lignocellulosic biomass can have a major impact on domestic transportation fuel supplies and thus help meet the Energy Independence and Security Act renewable energy goals (EISA 2007).

With gasification technology, biomass can be converted to gasoline via methanol synthesis and methanolto-gasoline (MTG) technologies. Producing a gasoline product that is infrastructure ready has much potential. Although the MTG technology has been commercially demonstrated with natural gas conversion, combining MTG with biomass gasification has not been shown. Therefore, a technoeconomic evaluation for a biomass MTG process based on currently available technology was developed to provide information about benefits and risks of this technology. The economic assumptions used in this report are consistent with previous U.S. Department of Energy Office of Biomass Programs technoeconomic assessments.

The feedstock is assumed to be wood chips at 2000 metric ton/day (dry basis). Two kinds of gasification technologies were evaluated: an indirectly-heated gasifier and a directly-heated oxygen-blown gasifier. The gasoline selling prices (2008 USD) excluding taxes were estimated to be $\$ 3.20 /$ gallon and $\$ 3.68 /$ gallon for indirectly-heated gasified and directly-heated. This suggests that a process based on existing technology is economic only when crude prices are above $\$ 100 / \mathrm{bbl}$. However, improvements in syngas cleanup combined with consolidated gasoline synthesis can potentially reduce the capital cost. In addition, improved synthesis catalysts and reactor design may allow increased yield. This is shown in the figure below (the ranges for the base case and the improved case reflect differences in gasifier types).

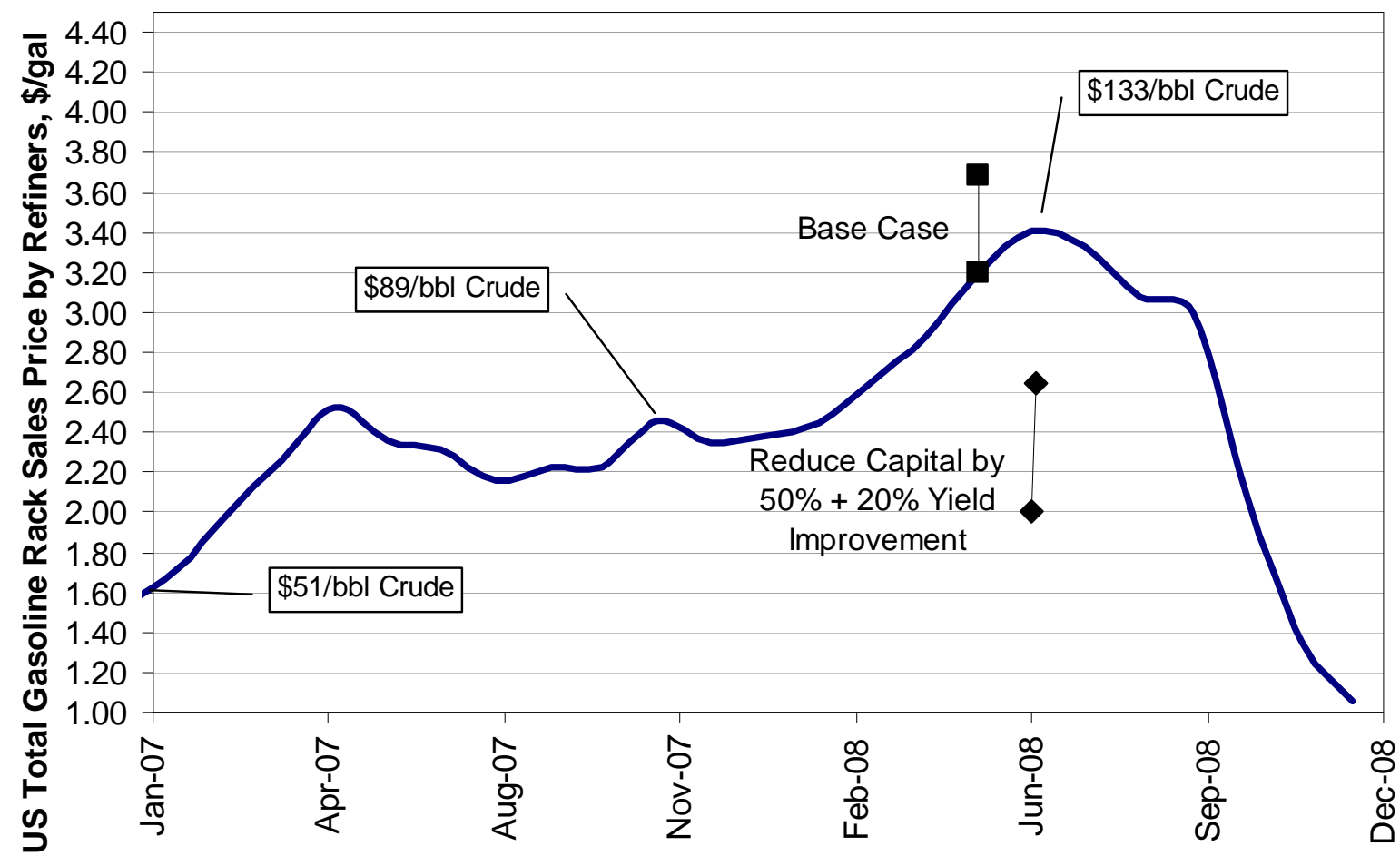




\section{Acknowledgment}

The authors thank DOE's biomass program for funding this work and acknowledge the modeling work performed by the National Renewable Energy Laboratory (NREL), which is publicly available and serves as the basis for gasification and syngas conditioning portion of the models. 


\section{Contents}

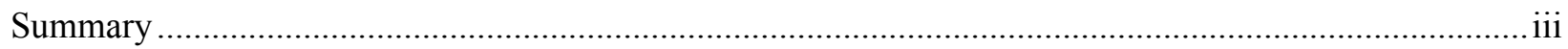

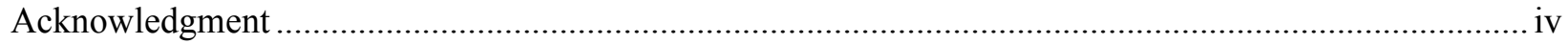

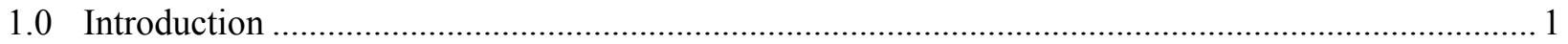

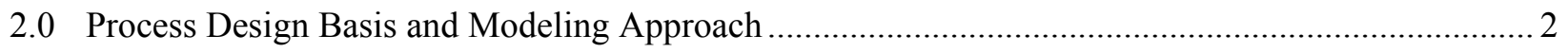

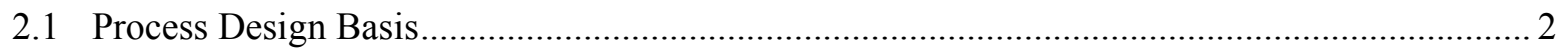

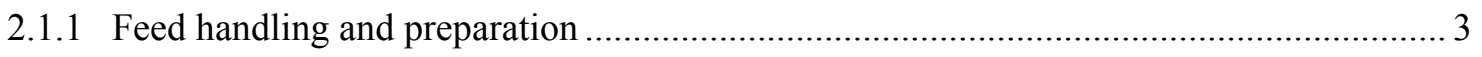

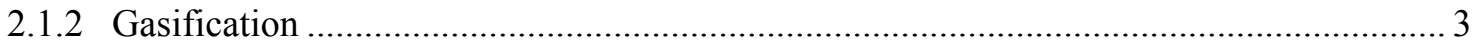

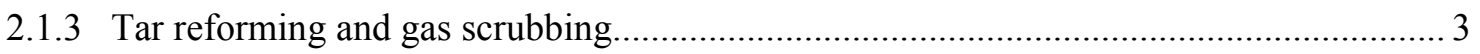

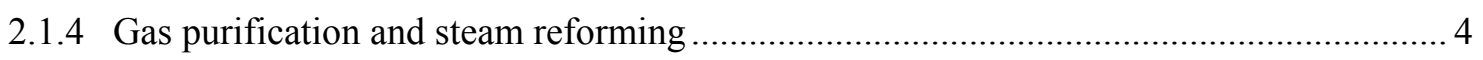

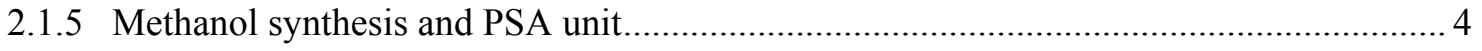

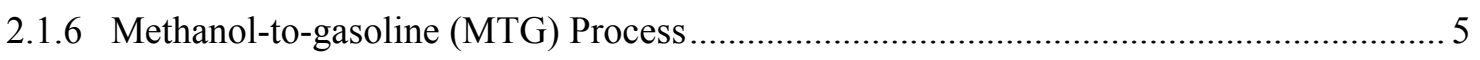

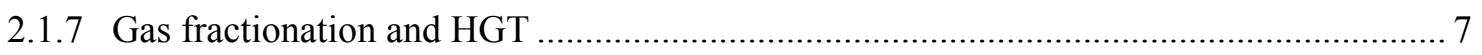

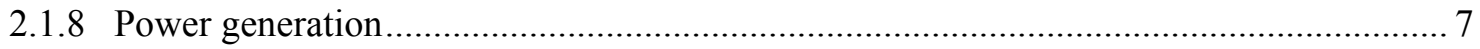

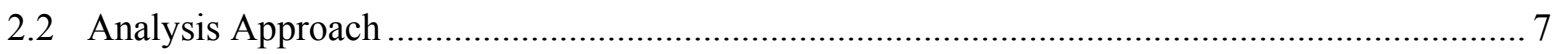

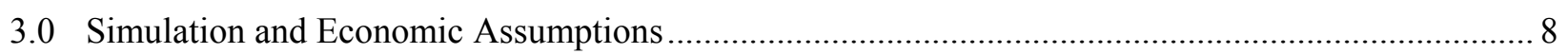

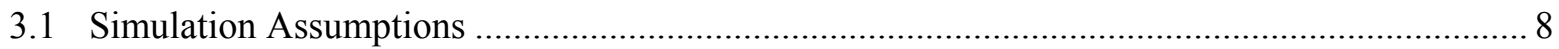

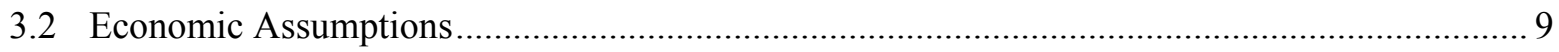

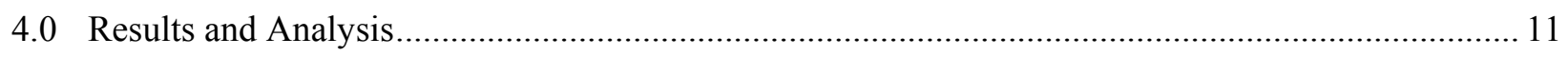

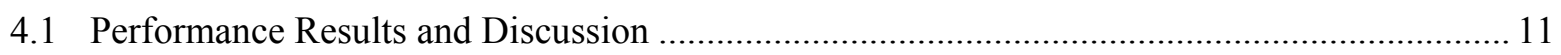

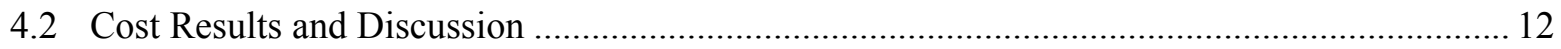

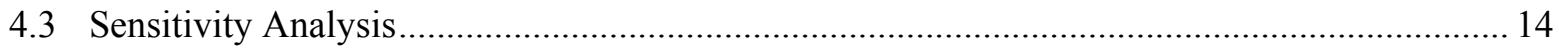

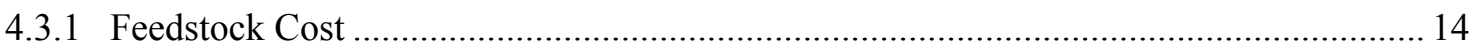

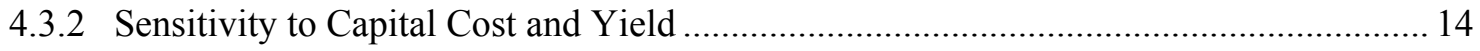

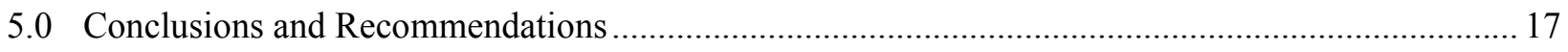

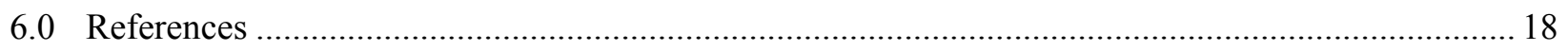

Appendix A. Heat and Material Balances for the Indirectly-heated Gasifier Case .................................20

Appendix B. Heat and Material Balances for the Directly-heated Gasifier Case ...................................... 34 


\section{Figures}

Figure 2-1 Block Diagram of Biomass-to-Gasoline via MTG System .................................................. 2

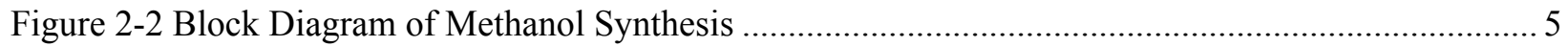

Figure 2-3 Block Diagram of Methanol-to-Gasoline Synthesis .......................................................... 6

Figure 4-1 US Refiners Gasoline Rack Sales Price and World Average Crude Prices (EIA, 2009) .......... 13

Figure 4-2 Effect of Feedstock Cost on Gasoline Price........................................................................... 14

Figure 4-3 Effect of Capital Cost on Gasoline Price.......................................................................... 15

Figure 4-4 Effect of Capital Cost Reduction and Yield Improvement .................................................... 16

Figure A-1 Process Flow Diagram for the Indirectly-heated Gasifier Case - Feed Prep through

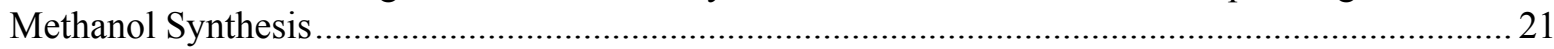

Figure A-2 Process Flow Diagram for the Indirectly-heated Gasifier Case - Gasoline Production........... 29

Figure B-1 Process Flow Diagram for the Directly-heated Gasifier Case - Feed Prep through

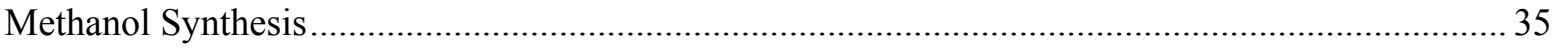

Figure B-2 Process Flows Diagram for the Directly-heated Gasifier Case - Gasoline Production............ 45

\section{Tables}

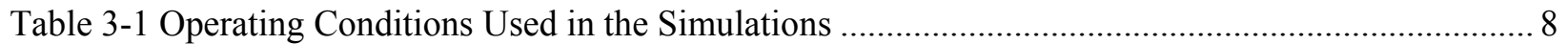

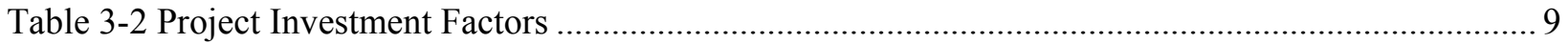

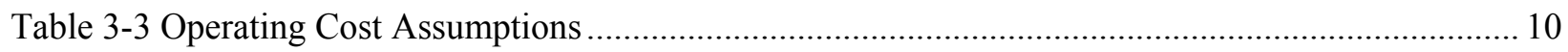

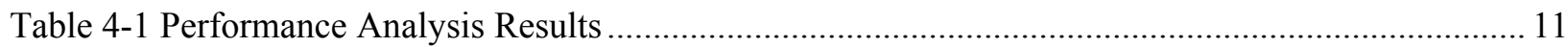

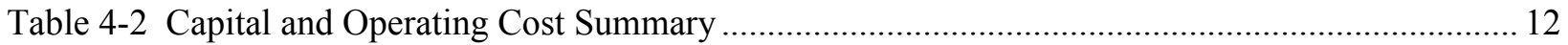

Table A-1 Steam Results for the Indirectly-heated Gasifier Case - Feed Prep through Methanol

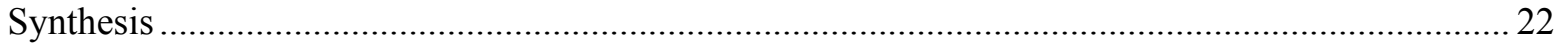

Table A-2 Steam Results for the Indirectly-heated Gasifier Case - Gasoline Production.......................... 30

Table B-1 Steam Results for the Directly-heated Gasifier Case - Feed Prep through Methanol

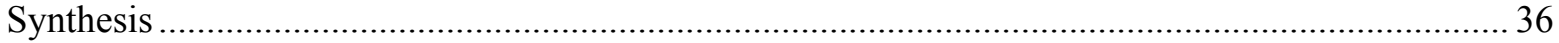

Table B-2 Steam Results for the Directly-heated Gasifier Case - Gasoline Production .......................... 46 


\subsection{Introduction}

Biomass is an important domestic resource that has the potential to make a significant impact on domestic fuel supplies and thus help meet the Energy Independence and Security Act renewable energy goals (EISA 2007). This study is part of an ongoing effort within the Department of Energy to meet the renewable energy goals for liquid transportation fuels. In particular, this report assesses the potential for gasoline production from biomass via the methanol-to-gasoline process.

Biomass conversion to synthesis gas by gasification has been demonstrated. The syngas, rich in $\mathrm{CO}$ and $\mathrm{H}_{2}$, can be further used to produce methanol, ethanol or other chemicals and liquid fuels. Among these products, methanol has been widely used as a solvent and also as the raw material for many chemicals and liquid fuels. In the 1970s, a methanol-to-gasoline (MTG) process was developed by Mobil in response to the Arab oil embargo (Chang, 1992). This technology was based on the direct conversion of methanol to hydrocarbons catalyzed by zeolite ZSM-5 (Olson, et al. 1981). A commercially operated gasto-gasoline plant using Mobil's MTG technology was built in New Zealand in the 1980s. In this plant, methane was converted into syngas by steam reforming, and then syngas was used to make methanol. The methanol was then converted to gasoline by a fixed-bed MTG process. Another type of MTG process is the fluid-bed type, which has not yet been commercially demonstrated (Chang, 1992). Currently, a coalbased MTG demonstration plant is under construction in China for start-up in late 2008/mid-2009 using MTG technology from ExxonMobil (Tabak, et al., 2008) and a commercial scale plant is being planned. ExxonMobil has also announced plans for two coal-to-gasoline plants in the United Stated using MTG technology (Ondry 2008, DKRW 2008). Although natural gas has been used and coal will be used for gasoline production, biomass feedstock has not yet been commercially used for MTG production.

A number of techno-economic assessments, using process design and simulation models, have been conducted for biomass gasification to fuels and chemicals such as methanol (Hamelinck and Faaij, 2001), Fischer-Tropsch liquid transportation fuels (Hamelinck, et al. 2003), hydrogen (Hamelinck and Faaij, 2001; Spath, et al. 2005), and ethanol (Aden, et al. 2005; Phillips, et al. 2007). Limited research and analysis work has been conducted for a biomass conversion to gasoline via MTG. In this study, process and economic models were developed for a biomass gasification based MTG system using existing technology. In the following sections, the design basis, simulation methods, and results are described. The sensitivity analysis results will also be discussed. 


\subsection{Process Design Basis and Modeling Approach}

A simplified block diagram for the Biomass MTG system is shown in Figure 2-1. In this system, wood chips are converted to synthesis gas in a gasifier. Raw syngas is sent to a tar reformer, a particulate scrubber, and finally a sulfur removal unit. A steam reformer is used to convert $\mathrm{CH}_{4}$ to $\mathrm{H}_{2}$ and $\mathrm{CO}$ and to adjust the $\mathrm{H}_{2} / \mathrm{CO}$ ratio to that required by methanol synthesis. Excess $\mathrm{CO}_{2}$ is removed in an amine unit. The syngas is then compressed and sent to the methanol synthesis section to produce feed for the MTG process. Part of the purge gas from methanol synthesis is used to produce hydrogen by a pressure swing adsorption (PSA) unit; the remainder purge gas is used as fuel. Raw methanol is converted to hydrocarbons and water in the MTG reactors. The raw gasoline product stream is separated to produce fuel gas, liquefied petroleum gas (LPG), light gasoline, and heavy gasoline. The heavy gasoline is further treated with hydrogen from the PSA to meet the final gasoline specifications. Steam generated in the processes is collected and sent to the steam cycle for power generation. Some steam is used in steam reforming and other processes.

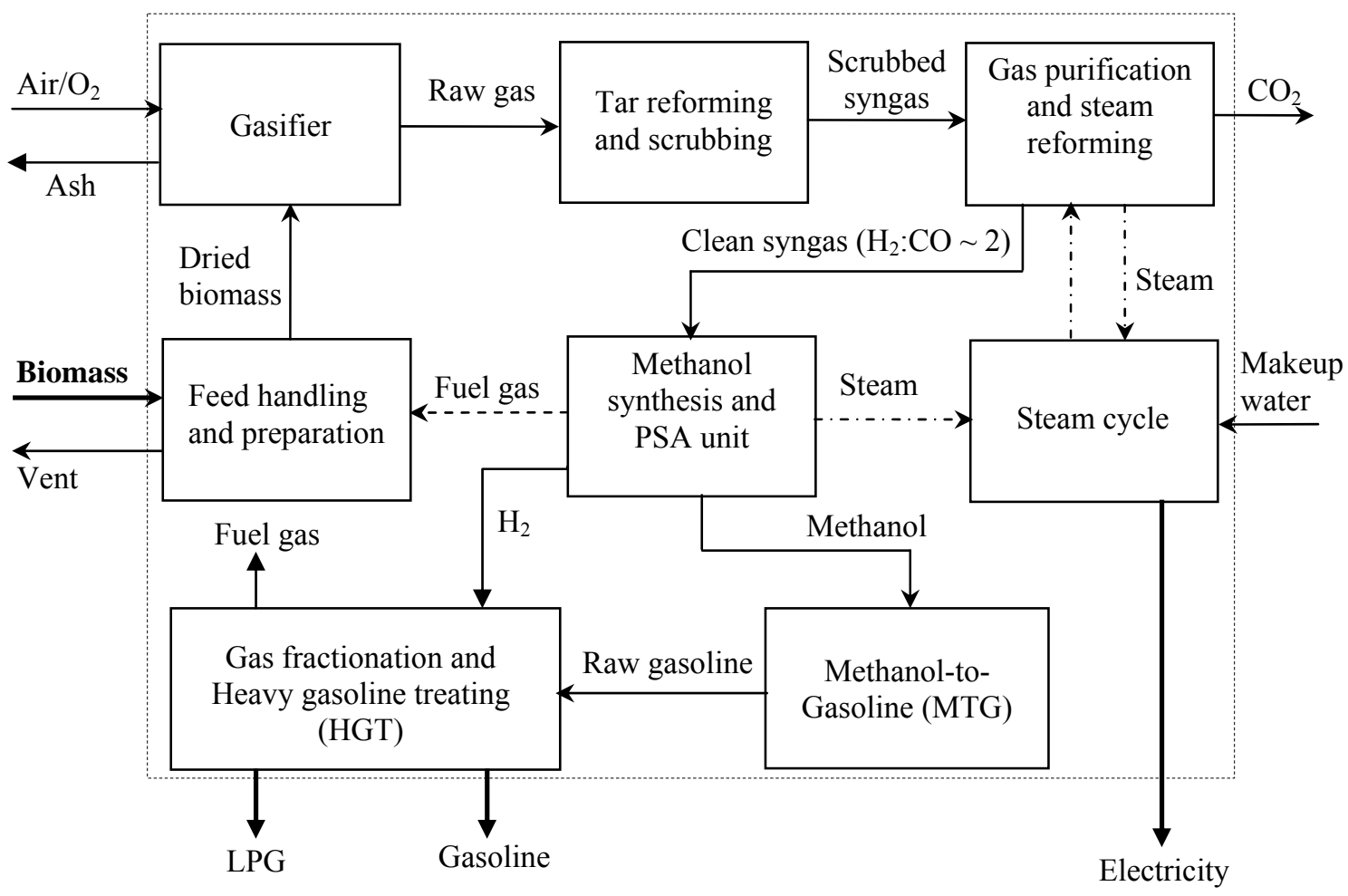

Figure 2-1 Block Diagram of Biomass-to-Gasoline via MTG System

\subsection{Process Design Basis}

The biomass-to-gasoline via MTG processing consists of eight main sections. Each section is briefly described in the follow paragraphs. Detailed process model diagrams and heat and material balances can be found in the Appendix. 


\subsubsection{Feed handling and preparation}

The feedstock is assumed to be wood chips at a moisture content of $50 \mathrm{wt} \%$. The wood feed rate is 2000 dry metric ton per day. The wet wood chips enter rotary dryers and are dried to a moisture content of 12 $\mathrm{wt} \%$ (Spath, et al. 2005). For the indirectly-heated gasifier based system, the hot flue gas from the char combustor is used for biomass drying. For the directly-heated gasifier, the char combustor flue gas is insufficient for feed drying. The dryer used for this gasifier is assumed to have an auxiliary burner to provide additional heat. Fuel for the burner comes from the methanol and MTG syntheses purge gas. The dried biomass is then conveyed to the gasifier.

\subsubsection{Gasification}

Two types of gasifiers are considered in this study: a low pressure indirectly-heated gasifier and a pressurized oxygen-blown, directly-heated gasifier. The indirectly-heated gasifier consists of two vessels: a gasifier and a combustor. Dried wood is fed into a low-pressure indirectly heated entrained flow gasifier. Steam extracted from the steam cycle is sent to the gasifier at a flow rate of $0.4 \mathrm{lb}$ of steam/lb of bone dry wood to fluidize the bed and to supply a portion of the heat required for the gasifier. The gasifier is assumed to be operated at $870^{\circ} \mathrm{C}\left(1598^{\circ} \mathrm{F}\right)$ and 23 psia. The gasifier is mainly heated by circulating olivine particles between the gasifier and the separate combustor. Olivine is synthetic sand that serves as the heat carrier in the gasifier. Char formed in the gasifier is carried out of the gasifier along with the olivine, separated in a series of cyclones and sent to the fluidized bed combustor, where air is used to burn the char, thereby reheating the olivine.

The oxygen-blown, directly-heated gasifier is a pressurized fluidized bed gasifier that also uses steam to fluidize the gasifer bed and to provide a portion of the heat. A pressurized cryogenic air separation unit provides purified oxygen at $99.5 \%$ for the gasifier at $350 \mathrm{psia}$ and $15.6^{\circ} \mathrm{C}\left(60^{\circ} \mathrm{F}\right)$. The mass flow rate of oxygen is varied to achieve an $870^{\circ} \mathrm{C}\left(1600^{\circ} \mathrm{F}\right)$ gasifier outlet stream temperature. The dried wood is fed using a lock hopper feeder system and pressurized by compressed $\mathrm{CO}_{2}$ recovered from the syngas purification process. The $\mathrm{CO}_{2}$ gas used in the lock hopper is fed at a flow rate of $0.03 \mathrm{lb}$ of $\mathrm{CO}_{2} / \mathrm{lb}$ dried wood and compressed to $330 \mathrm{psi}$. A small amount of $\mathrm{MgO}$ is also added to the gasifier to react with potassium in the ash to prevent agglomeration in the gasifier bed.

The indirectly-heated gasifier is modeled using the correlations reported in Spath, et al. (2005) which in turn is based on data from a Battelle-Columbus Laboratory (BCL) process development unit gasifier. The performance of the oxygen-blown, directly-heated pressurized gasifier was predicted using correlations derived from an experimental Institute of Gas Technology (IGT) gasifier (Evans, 1988).

\subsubsection{Tar reforming and gas scrubbing}

During gasification, a relatively small fraction of biomass is converted into tars consisting mostly of aromatic and poly-aromatic hydrocarbons. The nitrogen in the biomass is converted to ammonia. The raw syngas from the cyclone in the gasifier section is sent to a catalytic tar cracker, which is assumed to be a bubbling fluidized bed reactor. A portion of the syngas tar, methane, and other light hydrocarbons in the raw gas are converted to $\mathrm{CO}$ and $\mathrm{H}_{2}$, and some of the ammonia is converted to $\mathrm{N}_{2}$ and $\mathrm{H}_{2}$. The conversion percentage for each compound is reported in Spath, et al., (2005). The gas enters the tar reformer at the gasifier outlet temperature and exits the reformer at $750^{\circ} \mathrm{C}\left(1,383^{\circ} \mathrm{F}\right)$. The syngas is further 
cooled to $149^{\circ} \mathrm{C}\left(300^{\circ} \mathrm{F}\right)$ and sent to a wet scrubber to remove other impurities, such as particulates, residual ammonia and residual tars.

\subsubsection{Gas purification and steam reforming}

The scrubbed syngas is compressed to $450 \mathrm{psia}$. A liquid phase oxidation (LO-CAT) process followed by a $\mathrm{ZnO}$ bed is used to remove sulfur that would otherwise poison downstream catalysts. The LO-CAT process is assumed to remove the sulfur to a concentration of $10 \mathrm{ppm}_{2} \mathrm{~S}$, and then the $\mathrm{ZnO}$ bed polishes the syngas to less than 1 ppmv (Spath, et al. 2005).

Syngas leaving the $\mathrm{ZnO}$ bed is sent to a steam reformer to convert the remaining methane and light hydrocarbons to additional syngas and to adjust the $\mathrm{H}_{2}: \mathrm{CO}$ ratio via the water-gas shift reaction. The main steam reformer reactions are:

$$
\begin{gathered}
\mathrm{C}_{\mathrm{n}} \mathrm{H}_{\mathrm{m}}+\mathrm{nH}_{2} \mathrm{O} \leftrightarrow(\mathrm{n}+\mathrm{m} / 2) \mathrm{H}_{2}+\mathrm{nCO} \\
\mathrm{CO}+\mathrm{H}_{2} \mathrm{O} \leftrightarrow \mathrm{CO}_{2}+\mathrm{H}_{2}
\end{gathered}
$$

Before the syngas is sent to the steam reformer, it is mixed with high temperature steam from the steam cycle and the steam generated by the methanol synthesis reactor. Reactions take place between 800 and $900^{\circ} \mathrm{C}\left(1472\right.$ and $\left.1652^{\circ} \mathrm{F}\right)$. The steam reformer is fired with off-gas from the methanol synthesis section purge and the MTG syntheses section. The $\mathrm{H}_{2}$ : CO ratio is adjusted to $\sim 2$, as required by methanol synthesis reaction. The converted syngas passes through several heat exchangers to recover heat by generating saturate high pressure steam and superheated high pressure steam. The cooled syngas from the reforming process is further cooled by air cooling and cooling water. Excess $\mathrm{CO}_{2}$ is removed in an amine unit. The clean syngas is then compressed to 1450 psia.

\subsubsection{Methanol synthesis and PSA unit}

The compressed clean syngas is sent to a low-pressure methanol synthesis process. The principle reactions are:

$$
\begin{gathered}
\mathrm{CO}+2 \mathrm{H}_{2} \leftrightarrow \mathrm{CH}_{3} \mathrm{OH} \\
\mathrm{CO}_{2}+3 \mathrm{H}_{2} \leftrightarrow \mathrm{CH}_{3} \mathrm{OH}+\mathrm{H}_{2} \mathrm{O}
\end{gathered}
$$

The first reaction is the primary methanol synthesis reaction, and the second one represents a small fraction of carbon dioxide in the feed that acts as a promoter for the primary reaction.

A simplified process flow diagram of methanol synthesis is shown in Figure 2-2. 


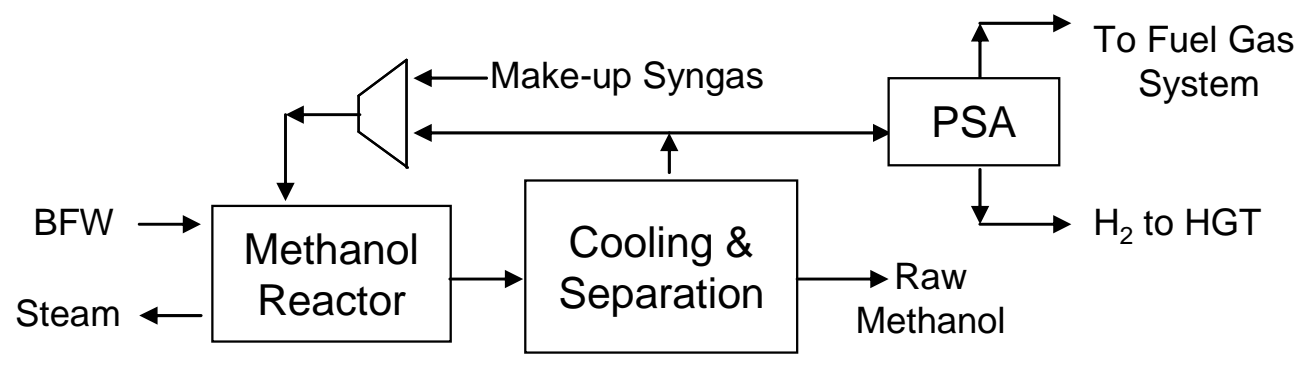

Figure 2-2 Block Diagram of Methanol Synthesis

The syngas from the steam reformer is compressed and preheated before entering the methanol reactor. Methanol synthesis temperatures and pressures typically range between 230 to $270^{\circ} \mathrm{C}\left(446\right.$ to $\left.578^{\circ} \mathrm{F}\right)$ and 735 to 1470 psia, respectively (Cheng, 1994, Fiedler, et al., 2000). The methanol reactor is assumed to be isothermal, low-pressure ( $850 \mathrm{psia}$ ), gas-phase shell and tube type with $\mathrm{ZnO} / \mathrm{CuO}$ catalyst in the tubes. The methanol synthesis reaction is highly exothermic and reaction heat is removed by generating medium pressure steam on the shell side of the reactor. The hot reactor product vapor is cooled by recycled compressed syngas and further cooled by air and then cooling water. The product stream is sent to a flash tank where liquid raw methanol is separated from the non-condensable gases. Approximately ninety-five percent of the vapor phase is compressed and recycled to the methanol reactor. The liquid product is further reduced in pressure to produce raw methanol at about $95 \mathrm{wt} \%$ purity. The raw methanol is sent to the MTG process. No distillation equipment is provided to produce high purity methanol.

Part of the purged gas from the methanol process is fed to a PSA unit where high purity $(99.9 \%)$ hydrogen is produced for use in the heavy gasoline treating (HGT) unit. This unit hydrotreats the heavy gasoline fraction from the MTG process and is described more fully in the next sections.

\subsubsection{Methanol-to-gasoline (MTG) Process}

Methanol is converted to gasoline by the Mobil MTG process. The principle reactions are (Chang, 1992, Cheng, 1994):

$$
\begin{aligned}
& \frac{\mathrm{n}}{2}\left[2 \mathrm{CH}_{3} \mathrm{OH} \Leftrightarrow \mathrm{CH}_{3} \mathrm{OCH}_{3}+\mathrm{H}_{2} \mathrm{O}\right] \stackrel{-\mathrm{nH}_{2} \mathrm{O}}{\longrightarrow} \mathrm{C}_{\mathrm{n}} \mathrm{H}_{2 \mathrm{n}} \rightarrow \mathrm{n}\left[\mathrm{CH}_{2}\right] \\
& {\left[\mathrm{CH}_{2}\right]=\text { average formula for the paraffin-aromatic mixture }}
\end{aligned}
$$

First methanol is partially dehydrated to dimethyl ether (DME) using a methanol dehydration catalyst. This reaction is followed by olefin formation and finally aromatic/paraffin formation over a zeolite catalyst (ZSM-5). The final hydrocarbon product is predominantly in the gasoline boiling range, with some LPG and fuel gas generated as well.

Mobil has developed two reactor configurations: an adiabatic fixed-bed and a circulating fluidized-bed process (Kaneko, et al. 2002). ZSM-5 undergoes deactivation with time. In fixed bed reactors, the catalyst ages in a "band-aging" mechanism that travels down the length of the reactor. Methanol breakthrough occurs when the age-band reaches the bottom of the reactor and indicates the need for catalyst regeneration. Regeneration is accomplished by burning off organic deposits with air (Cheng, 1994). 
Circulating fluidized beds have the potential to simplify heat and catalyst activity management by providing continuous catalyst regeneration.

The fixed bed concept was demonstrated on a commercial scale in New Zealand using natural gas as the feed stock. This plant produced $14,500 \mathrm{bpd}$ of gasoline and used one DME reactor and four MTG reactors operating in parallel, with a fifth MTG reactor off-line for regeneration. Multiple reactors minimize bed pressure drop and allow a staggered operating sequence to prevent significant methanol breaking through to the product (Cheng, 1994). The fluidized bed configuration has been demonstrated on a pilot scale only.

The simulation of the MTG process for this work is based on a study by the W.R. Grace Co. (1982 a,b,c) using the fixed bed process. A simplified flow diagram is shown in Figure 2-3.

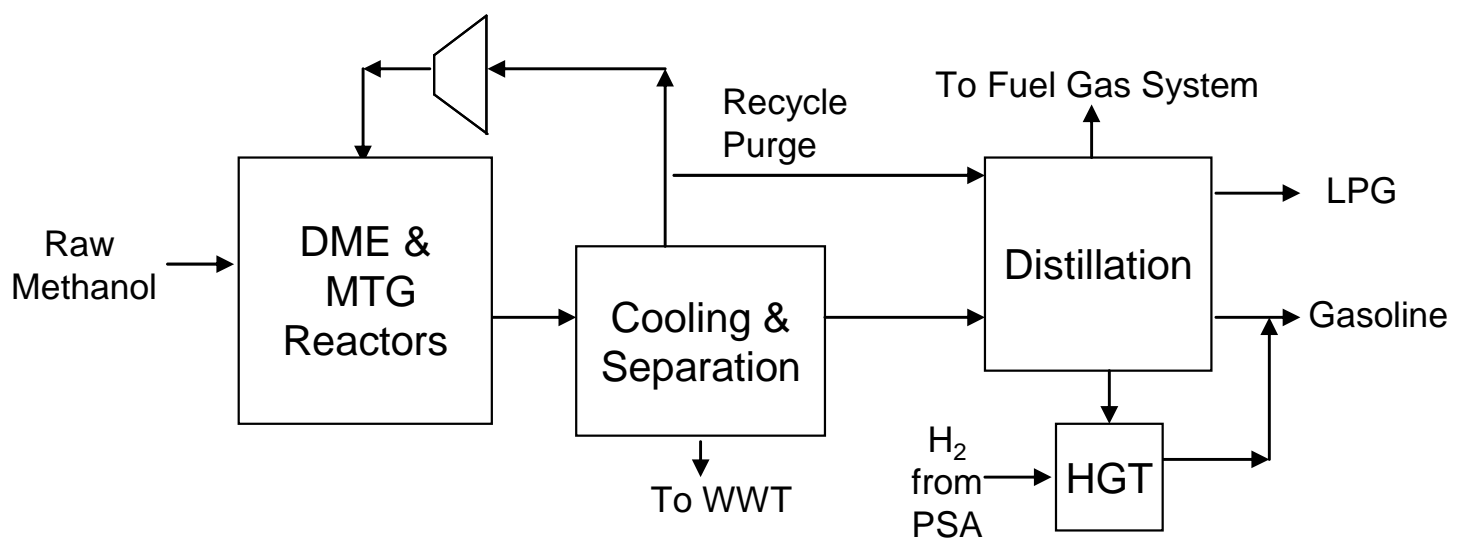

Figure 2-3 Block Diagram of Methanol-to-Gasoline Synthesis

The MTG process is highly exothermic and heat management is performed in two steps. First, raw methanol is sent to a single fixed bed dehydration reactor to convert some of the methanol to dimethyl ether (DME) and water. Pre-conversion to DME reduces the exotherm in the next set of reactors. The DME reactor effluent is combined with recycle gas and sent to two conversion reactors operating in parallel. A third reactor is offline for catalyst regeneration. The mixture is converted to hydrocarbons and water over a zeolite catalyst in the conversion reactors. The reaction is highly exothermic and is controlled by using a large recycle gas-to-DME reactor effluent ratio. Approximately ninety-nine percent of the non-condensable gas is combined with the effluent from the dehydration unit and sent to the conversion unit to achieve a recycle ratio of 9 moles of recycle gas per mole of methanol feed (Cheng, 1994). The remaining gas is combined with the hydrocarbon liquid and sent to the gas fractionation unit.

The hydrocarbon product typically yields about $85 \mathrm{wt} \%$ high-quality gasoline. Almost no hydrocarbons with carbon numbers greater than ten are found in the product because of the shape selective nature of the conversion catalyst (Chang, 1992). The process operates at essentially 100\% conversion of methanol until the catalyst is deactivated by carbon formation. The deactivated catalyst is regenerated by burning off the coke from the catalyst using air combustion. 


\subsubsection{Gas fractionation and HGT}

The hydrocarbons from the MTG process are separated in a gas fractionation unit to produce fuel gas, liquefied petroleum gas (LPG), light gasoline and a heavy gasoline feed stream. The gas fractionation unit consists of three fractionation towers, the de-ethanizer, the de-butanizer, and the gasoline splitter.

The product stream from the MTG process gas-liquid separator is first sent to a de-ethanizer tower to remove ethane and lighter hydrocarbons for fuel gas from the product. The de-ethanizer bottoms are then fractionated in the debutanizer to produce the feed to the gasoline splitter. The de-butanizer produces a light over head consisting of $\mathrm{C} 4$ and lower hydrocarbons, which is suitable for sale as liquefied petroleum gas (LPG). Most of the de-butanizer bottoms (a stream containing C5+ hydrocarbon) are sent to the gasoline splitter. A small portion of the de-butanizer column bottoms is recycled to the de-ethanizer column to act as a lean oil solvent. This assists in the separation of the light gases from the liquids in the de-ethanizer. In the gasoline splitter, light gasoline containing hydrocarbons in the C5 to C9 range are separated in the overhead flow. The light gasoline is cooled and then sent to gasoline storage as gasoline product. The bottom flow from the gasoline splitter is heavy gasoline with hydrocarbons numbering C9 and above. This heavy fraction contains durene, a low melting point component, which if not removed, can cause freeze point problems in the gasoline. The heavy gasoline is treated in the Heavy Gasoline Treater (HGT).

The HGT unit removes durene via hydrogenation. The hydrogen produced from the PSA unit is combined with the heavy gasoline feed from the gasoline splitter. The mixture is sent to the catalytic hydrotreating reactor. The effluent is cooled by process streams and air before it is sent to the gas-liquid separator. Most of the vapor is compressed and recycled to the hydrotreater, with a small purge going to fuel gas system. The liquid flow from the separator is stabilized in the product stripper. The stablilized heavy gasoline is combined with the lighter gasoline cut from the de-butanizer, cooled and sent to product storage.

In the indirectly-heated gasifier case, some of the LPG is used as fuel for the steam reformer. The remaining LPG is available for sale as a by-product. In the directly-heated gasifier case, all of the LPG is used as fuel for the steam reformer and the wood dryer.

\subsubsection{Power generation}

Saturated steam is generated by cooling process streams in the gasifier, steam reformer and MTG synthesis areas. It is then superheated and sent to a steam turbine to generate power for the entire plant. Excess power is sold. The steam cycle also provides medium and low pressure process steam for use in the system.

\subsection{Analysis Approach}

The process simulation for the gasifier based biomass-to-gasoline via MTG system was developed in CHEMCAD 6.1. The capital and operating costs were assembled in an EXCEL spreadsheet using information from the CHEMDAD simulation. A discounted cash flow method was used to estimate the product selling price. 


\subsection{Simulation and Economic Assumptions}

This section describes the performance and cost simulation assumptions.

\subsection{Simulation Assumptions}

Table 3-1 shows the main assumptions for the indirectly-heated and directly-heated gasifier based cases.

Table 3-1 Operating Conditions Used in the Simulations

\begin{tabular}{|c|c|c|}
\hline \multicolumn{3}{|c|}{ Gasification } \\
\hline Gasifier Type & Indirectly-Heated Gasifier & Directly-Heated Gasifier \\
\hline Gasifier Pressure, psia & 23 & 330 \\
\hline Gasifier Temperature, ${ }^{\circ} \mathrm{C}\left({ }^{\circ} \mathrm{F}\right)$ & $870(1598)$ & $871(1600)$ \\
\hline Biomass Feed, metric ton/d, dry basis & 2000 & 2000 \\
\hline Oxidant & Air & Oxygen \\
\hline Dried Biomass, moisture fraction $\%$ & 12 & 12 \\
\hline Tar Reformer, ${ }^{\circ} \mathrm{C}\left({ }^{\circ} \mathrm{F}\right) / \mathrm{P}$, psia & $717(1323) / 20$ & $717(1323) / 327$ \\
\hline \multicolumn{3}{|c|}{ Steam Reforming } \\
\hline Reformer outlet temperature, ${ }^{\circ} \mathrm{C}\left({ }^{\circ} \mathrm{F}\right)$ & $900(1652)$ & $900(1652)$ \\
\hline Reformer outlet pressure, psia & 423 & 430 \\
\hline Reformed gas $\mathrm{H}_{2} / \mathrm{CO}$ & 2.1 & 2.1 \\
\hline \multicolumn{3}{|c|}{ Methanol Synthesis and Purification } \\
\hline Reactor outlet temperature, ${ }^{\circ} \mathrm{C}\left({ }^{\circ} \mathrm{F}\right)$ & $260(500)$ & $260(500)$ \\
\hline Reactor outlet pressure, psia & 840 & 840 \\
\hline $\mathrm{kg} / \mathrm{h}$ methanol/L catalyst & 0.9 & 0.9 \\
\hline \multicolumn{3}{|c|}{ Methanol-to-Gasoline } \\
\hline \multicolumn{3}{|l|}{ Dehydration unit (DME reactor) } \\
\hline Outlet temperature, ${ }^{\circ} \mathrm{C}\left({ }^{\circ} \mathrm{F}\right)$ & $400(752)$ & $400(752)$ \\
\hline Outlet pressure, psia & 385 & 385 \\
\hline $\mathrm{kg} / \mathrm{h}$ hydrocarbon/L catalyst & 0.96 & 0.96 \\
\hline \multicolumn{3}{|l|}{ Conversion unit (MTG reactor) } \\
\hline Outlet temperature, ${ }^{\circ} \mathrm{C}\left({ }^{\circ} \mathrm{F}\right)$ & $390(733)$ & $390(733)$ \\
\hline Outlet pressure, psia & 300 & 300 \\
\hline $\begin{array}{l}\text { Recycle ratio: moles recycle gas } \\
\text { /mole methanol }\end{array}$ & 9 & 9 \\
\hline $\mathrm{kg} / \mathrm{h}$ hydrocarbon/L catalyst & 0.25 & 0.25 \\
\hline \multicolumn{3}{|c|}{ Heavy Gasoline Treating } \\
\hline Reactor outlet temperature, ${ }^{\circ} \mathrm{C}\left({ }^{\circ} \mathrm{F}\right)$ & $282(540)$ & $282(540)$ \\
\hline Reactor outlet pressure, psia & 470 & 470 \\
\hline $\begin{array}{l}\mathrm{H}_{2} \text { partial pressure at reactor inlet, } \\
\text { psia }\end{array}$ & 423 & 423 \\
\hline
\end{tabular}




\subsection{Economic Assumptions}

Most of the base equipment costs for gas purification and conditioning and steam cycle and power generation sections of the plant were obtained from Spath, et al. (2005). The estimation of the equipment cost for the two types of gasifiers is based on Hamelinck and Faaij (2002). Capital costs for methanol synthesis and methanol-to-gasoline synthesis were estimated using ASPEN ICARUS 2006.5 and the W.R. Grace Report (Grace, 1982 a,b,c).

All capital costs are reported in 2008 dollars. Equipment cost escalation is calculated by using the Chemical Engineering Plant Cost Index (CEPCI). The total capital investment is factored from installed equipment costs as shown in Table 3-2. Table 3-3 lists the assumptions used to estimate the production costs.

Table 3-2 Project Investment Factors

\begin{tabular}{|l|c|}
\hline & \% of TPEC \\
\hline Total Purchased Equipment Cost (TPEC) & $100 \%$ \\
\hline Purchased Equipment Installation & $39 \%$ \\
\hline Instrumentation and Controls & $26 \%$ \\
\hline Piping & $31 \%$ \\
\hline Electrical Systems & $10 \%$ \\
\hline Buildings (including services) & $29 \%$ \\
\hline Yard Improvements & $12 \%$ \\
\hline Total Installed Cost (TIC) & $247 \%$ \\
\hline Indirect Costs & \\
\hline Engineering & $32 \%$ \\
\hline Construction & $34 \%$ \\
\hline Legal and Contractors Fees & $23 \%$ \\
\hline Project Contingency & $37 \%$ \\
\hline Total Indirect & $126 \%$ \\
\hline Total Project Investment & $373 \%$ \\
\hline
\end{tabular}


Table 3-3 Operating Cost Assumptions

\begin{tabular}{|c|c|c|c|}
\hline & $\begin{array}{l}\text { Value used } \\
\text { in model, } \\
2008 \text { basis }\end{array}$ & Units or Basis & Reference \\
\hline \multicolumn{4}{|l|}{ Raw Materials } \\
\hline Hybrid poplar chips & 60 & \$/dry short ton & Aden, 2008 \\
\hline Olivine makeup & 258 & $\$ /$ short ton & Phillips, et al. 2007* \\
\hline Ash disposal & 44 & $\$ /$ short ton & Phillips, et al. 2007* \\
\hline Tar cracker catalyst & 8.06 & $\$ / 1 b$ & Phillips, et al., 2007* \\
\hline Reformer catalyst & 25.4 & $\$ / 1 b$ & SRI PEP 2007* \\
\hline Methanol catalyst & 9.69 & $\$ /$ lb catalyst & SRI PEP 2007 \\
\hline MTG zeolite catalyst & 60 & $\$ /$ lb catalyst & SRI PEP 2007 \\
\hline DME catalyst & 11.6 & $\$ /$ lb catalyst & SRI PEP 2007 \\
\hline \multicolumn{4}{|l|}{ Utilities } \\
\hline Waste water treatment & 2.4 & $\$ / 100 \mathrm{ft}^{3}$ & Phillips, et al. 2007 \\
\hline Cooling tower makeup & 245 & $\notin / 1000$ gal & Phillips, et al. 2007 \\
\hline Electricity & 6.68 & $\phi / \mathrm{kWh}$ & EIA, 2008b \\
\hline \multicolumn{4}{|l|}{ Economic Assumptions } \\
\hline Stream Factor & $90 \%$ & & estimated \\
\hline MACRS Depreciation, yrs & 7 & & Phillips, et al. 2007 \\
\hline Plant life, yrs & 20 & & Phillips, et al. 2007 \\
\hline $\begin{array}{l}\text { Construction Period } \\
1^{\text {st }} 6 \text { months expenditure } \\
\text { Next } 12 \text { months expenditure } \\
\text { Last } 12 \text { months expenditure }\end{array}$ & $\begin{array}{c}2.5 \text { years } \\
8 \% \\
60 \% \\
32 \% \\
\end{array}$ & & Phillips, et al. 2007 \\
\hline $\begin{array}{l}\text { Start-up time } \\
\text { Revenues } \\
\text { Variable Costs } \\
\text { Fixed Costs }\end{array}$ & $\begin{array}{c}6 \text { months } \\
50 \% \\
75 \% \\
100 \%\end{array}$ & & Phillips, et al. 2007 \\
\hline Working Capital & \multicolumn{2}{|c|}{$5 \%$ of Total Capital Investment } & Phillips, et al. 2007 \\
\hline Land & \multicolumn{2}{|c|}{$\begin{array}{l}6 \% \text { of Total Purchased Equipment } \\
\text { Cost (taken as } 1^{\text {st }} \text { year construction } \\
\text { expense) }\end{array}$} & Phillips, et al. 2007 \\
\hline Internal Rate of Return & \multicolumn{2}{|l|}{$10 \%$} & Phillips, et al. 2007 \\
\hline
\end{tabular}




\subsection{Results and Analysis}

This section describes the main performance and cost simulation results for the biomass-to-gasoline via MTG systems. The results of the sensitivity analysis are also discussed.

\subsection{Performance Results and Discussion}

Table 4-1 shows the main performance results for the biomass-to-gasoline via MTG systems.

Table 4-1 Performance Analysis Results

\begin{tabular}{|c|c|c|}
\hline Case & Indirectly-Heated Gasifier & Directly-Heated Gasifier \\
\hline Gasifier pressure, psi & 23 & 330 \\
\hline Gasifier temperature, ${ }^{\circ} \mathrm{C}\left({ }^{\circ} \mathrm{F}\right)$ & $870(1598)$ & $871(1600)$ \\
\hline $\mathrm{H}_{2}: \mathrm{CO}$ ratio in scrubbed syngas, molar & 0.9 & 1.4 \\
\hline Char production, $\mathrm{lb} / \mathrm{hr}$ & 33970 & 7510 \\
\hline \multicolumn{3}{|l|}{ Feed } \\
\hline Wood chips, metric ton/d, dry basis & 2000 & 2000 \\
\hline Natural Gas, lb/hr & -- & -- \\
\hline \multicolumn{3}{|l|}{ Feed Preparation } \\
\hline Directly-heated dryer & $\begin{array}{c}\text { Heat from char burner } \\
\text { exhaust only }\end{array}$ & $\begin{array}{l}\text { Heat from char burner exhaust } \\
\text { plus auxiliary fuel gas burner }\end{array}$ \\
\hline \multicolumn{3}{|l|}{ Products } \\
\hline Gasoline, mmgal/y & 40 & 40 \\
\hline $\mathrm{LPG}, \mathrm{mmgal} / \mathrm{y}$ & 7.2 & -- \\
\hline \multicolumn{3}{|l|}{ Methanol Synthesis } \\
\hline $\begin{array}{l}\text { Recycle ratio: moles recycle gas } \\
\text { /moles makeup gas }\end{array}$ & 5.6 & 2.0 \\
\hline \multicolumn{3}{|l|}{ Power Consumption, MW } \\
\hline Air separation unit & -- & -8.6 \\
\hline Lock hopper gas compressor & -- & -0.2 \\
\hline Dryer air blower & -- & -0.1 \\
\hline Char burner air compressor & -4.8 & -0.5 \\
\hline Syngas compressor & -16.0 & -1.9 \\
\hline Reformer air compressor & -1.1 & -1.8 \\
\hline Reformer flue gas blower & -0.5 & -0.6 \\
\hline Clean syngas compressor & -3.8 & -4.6 \\
\hline Methanol syn. recycle compressor & -2.7 & -2.4 \\
\hline MTG recycle \& hydrogen compressor & -3.0 & -3.7 \\
\hline Steam turbines & 24.4 & 56.0 \\
\hline Net power, MW & -7.5 & 32 \\
\hline Carbon Efficiency, \% & $32 \%$ & $29 \%$ \\
\hline $\begin{array}{l}\text { Thermal Efficiency, \%, higher heating } \\
\text { value (HHV) basis }\end{array}$ & $47 \%$ & $41 \%$ \\
\hline
\end{tabular}


In general, one would expect that the directly-heated gasifier would produce more final product than an indirectly-heated gasifier due to the former's lower char production and thus higher syngas rate.

However, the biomass dryer for the directly-heated gasifier needs an external burner to supply the dryer heat load. Fuel to the auxiliary burner is supplied by using all of the LPG plus a higher purge rate in the methanol synthesis step. The higher methanol off-gas purge rate reduces the yield of methanol and hence reduces the final gasoline yield. This effect could be mitigated by 1) using natural gas in the dryer burner, or 2) by using an indirectly-heated dryer using steam as the heat source. Analysis of these options is beyond the scope of this work. Lack of LPG product in the directly-heated gasifier case reduces the carbon and the thermal efficiency as compared to the indirectly-heated gasifier case. However, the larger volumes of gas through the syngas generation and cleanup steps greatly increase the amount of steam recuperated, and thus results in a net export of power.

\subsection{Cost Results and Discussion}

Table 4-2 shows the capital and operating cost breakdown for each section of the plant.

Table 4-2 Capital and Operating Cost Summary

\begin{tabular}{|c|c|c|c|c|}
\hline Case & \multicolumn{2}{|c|}{ Indirectly-Heated Gasifier } & \multicolumn{2}{|c|}{ Directly-Heated Gasifier } \\
\hline CAPITAL COSTS & Million \$ & $\%$ of Total & Million \$ & $\%$ of Total \\
\hline Air separation unit & -- & -- & $\$ 10$ & $8 \%$ \\
\hline Feed prep and drying & $\$ 11$ & $11 \%$ & $\$ 12$ & $9 \%$ \\
\hline $\begin{array}{l}\text { Gasification with tar reforming, heat } \\
\text { recovery, scrubbing }\end{array}$ & $\$ 17$ & $16 \%$ & $\$ 41$ & $31 \%$ \\
\hline Syngas cleanup \& compression & $\$ 33$ & $32 \%$ & $\$ 27$ & $20 \%$ \\
\hline Methanol synthesis & $\$ 10$ & $10 \%$ & $\$ 8$ & $6 \%$ \\
\hline MTG, gas fractionation, and HGT & $\$ 24$ & $23 \%$ & $\$ 24$ & $16 \%$ \\
\hline Steam system and power generation & $\$ 7$ & $7 \%$ & $\$ 11$ & $8 \%$ \\
\hline Remainder off-site battery limits (OSBL) & $\$ 2$ & $2 \%$ & $\$ 2$ & $1 \%$ \\
\hline Total Purchased Equipment Cost (TPEC) & $\$ 104$ & $100 \%$ & $\$ 136$ & $100 \%$ \\
\hline Total Installed Cost (TIC), mm\$ & \multicolumn{2}{|c|}{$\$ 258$} & \multicolumn{2}{|c|}{$\$ 335$} \\
\hline Total Indirect Cost, mm\$ & \multicolumn{2}{|c|}{$\$ 132$} & \multicolumn{2}{|c|}{$\$ 272$} \\
\hline Total Project Investment, mm\$ & \multicolumn{2}{|c|}{$\$ 383$} & \multicolumn{2}{|c|}{$\$ 499$} \\
\hline OPERATING COSTS & $\$ /$ gal & $\%$ of total & $\$ /$ gal & $\%$ of total \\
\hline Biomass & 1.07 & $34 \%$ & 1.07 & $29 \%$ \\
\hline Natural Gas & 0.00 & $0 \%$ & 0.00 & $0 \%$ \\
\hline Catalysts \& Chemicals & 0.29 & $9 \%$ & 0.38 & $10 \%$ \\
\hline Waste Disposal & 0.04 & $1 \%$ & 0.05 & $1 \%$ \\
\hline Electricity and other utilities & 0.13 & $4 \%$ & -0.38 & $-10 \%$ \\
\hline LPG credit & -0.30 & $-9 \%$ & 0.00 & $0 \%$ \\
\hline Fixed Costs & 0.50 & $16 \%$ & 0.61 & $17 \%$ \\
\hline Capital Depreciation & 0.47 & $15 \%$ & 0.62 & $17 \%$ \\
\hline Average Income Tax & 0.33 & $10 \%$ & 0.43 & $12 \%$ \\
\hline Average Return on Investment & 0.65 & $20 \%$ & 0.91 & $25 \%$ \\
\hline $\begin{array}{l}\text { Estimated Selling Price at } 10 \% \text { ROI, } \\
\$ / \text { gal gasoline }\end{array}$ & 3.20 & $100 \%$ & 3.68 & $100 \%$ \\
\hline
\end{tabular}


Methanol and MTG synthesis, gas fractionation, and HGT represents about one-third of the total equipment cost. The total capital cost of the directly-heated gasifier based system is about $30 \%$ higher than that of the indirectly-heated gasifier based system. The directly-heated gasifier is pressurized and requires an Air Separation Unit (ASU) whereas the indirectly-heated gasifier is at atmospheric pressure.

For both gasifier cases, approximately $30 \%$ of the production cost is feedstock related. The indirectlyheated gasifier based system has by-products credit of LPG and no export electricity. The directly-heated gasifier has credits from electricity production, and no LPG credit. Therefore, the selling price of gasoline for the indirectly-heated gasifier based system is lower than that of the directly-heated gasifier based system.

As shown in Figure 4-1, the gasoline refiners rack sales prices from January 2007 to December 2008 ranged from a low in December 2008 of $\$ 1.05 /$ gallon to a high of $\$ 3.41 /$ gallon in June 2008 . The June 2008 gasoline price corresponds to the EIA reported peak of over \$130/barrel (bbl) crude oil.

Superimposed on this plot is the estimated selling price for the indirectly-heated gasifier case (horizontal line at $\$ 3.20$ /gallon). The estimated gasoline selling prices for the two gasifier models are at or above the high end of the rack market price as shown in Figure 4-1. Thus, the base case gasification and MTG technology investigated in this study appears to be economically viable only at crude prices above $\$ 100 / \mathrm{bbl}$. To give this another perspective, if taxes are assumed to be an additional $25 \%$ on top of the rack price, then the pump price for the base case indirectly-heated gasifier is over $\$ 4.25 /$ gallon.

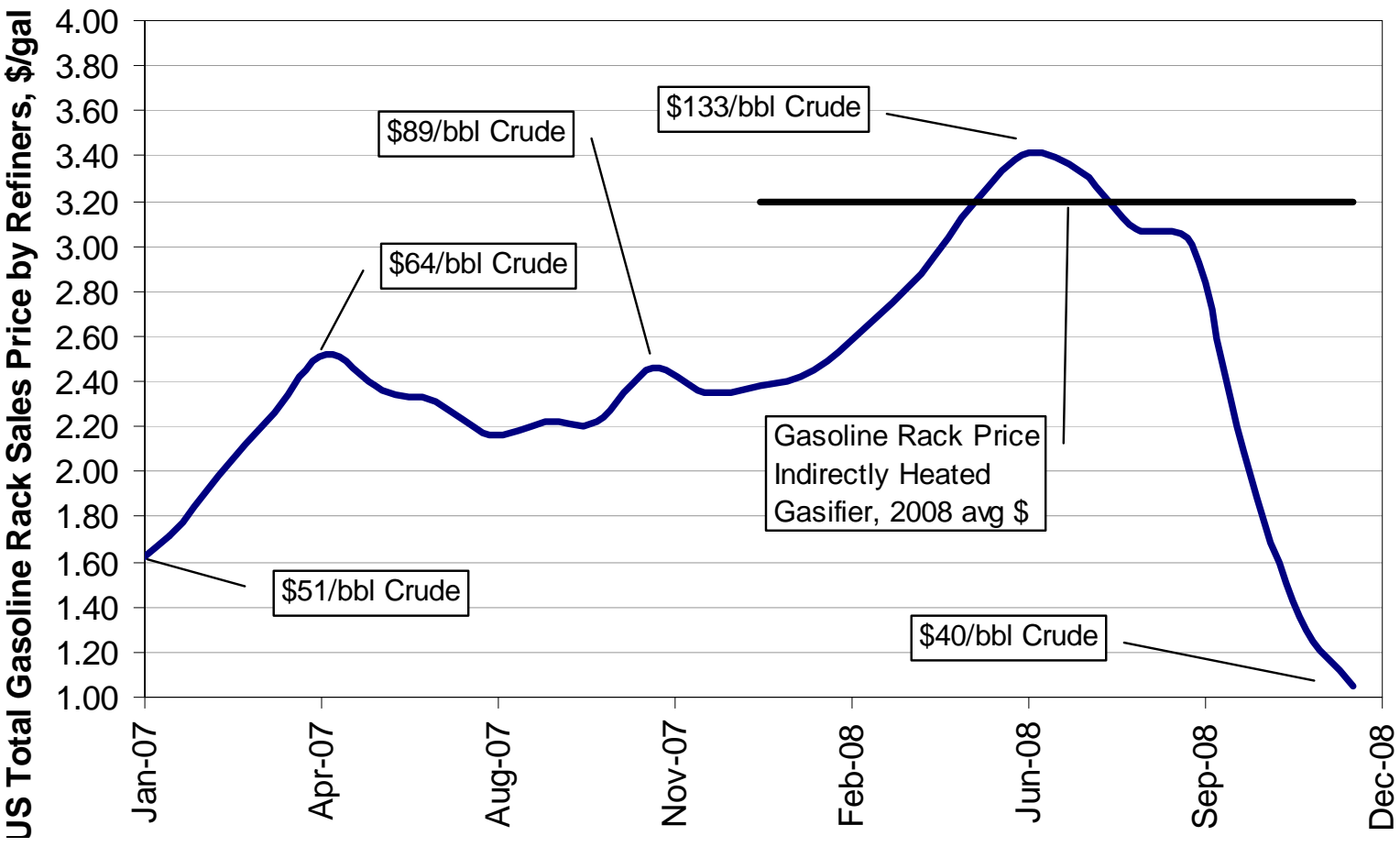

Figure 4-1 US Refiners Gasoline Rack Sales Price and World Average Crude Prices (EIA, 2009) 


\subsection{Sensitivity Analysis}

A sensitivity analysis was conducted to investigate the effects of different cost assumptions. The feedstock cost, total capital cost and yield were varied to determine their economic impact. The feedstock cost was chosen because it represents between $30 \%$ of the total production cost as shown in Table 4-2. The capital cost was chosen because it has potential for cost reductions.

\subsubsection{Feedstock Cost}

Figure 4-2 shows a range of feedstock prices superimposed on the rack gasoline sale price. For each price range, the upper point corresponds to the directly heated gasifier. Very low feedstock costs (below $\$ 20 /$ dry ton) are required to approach the medium gasoline rack price for this time frame. As little or no feedstock is available at this price, this effect alone suggests that producing gasoline with current technology will never be viable unless crude prices are extremely high.

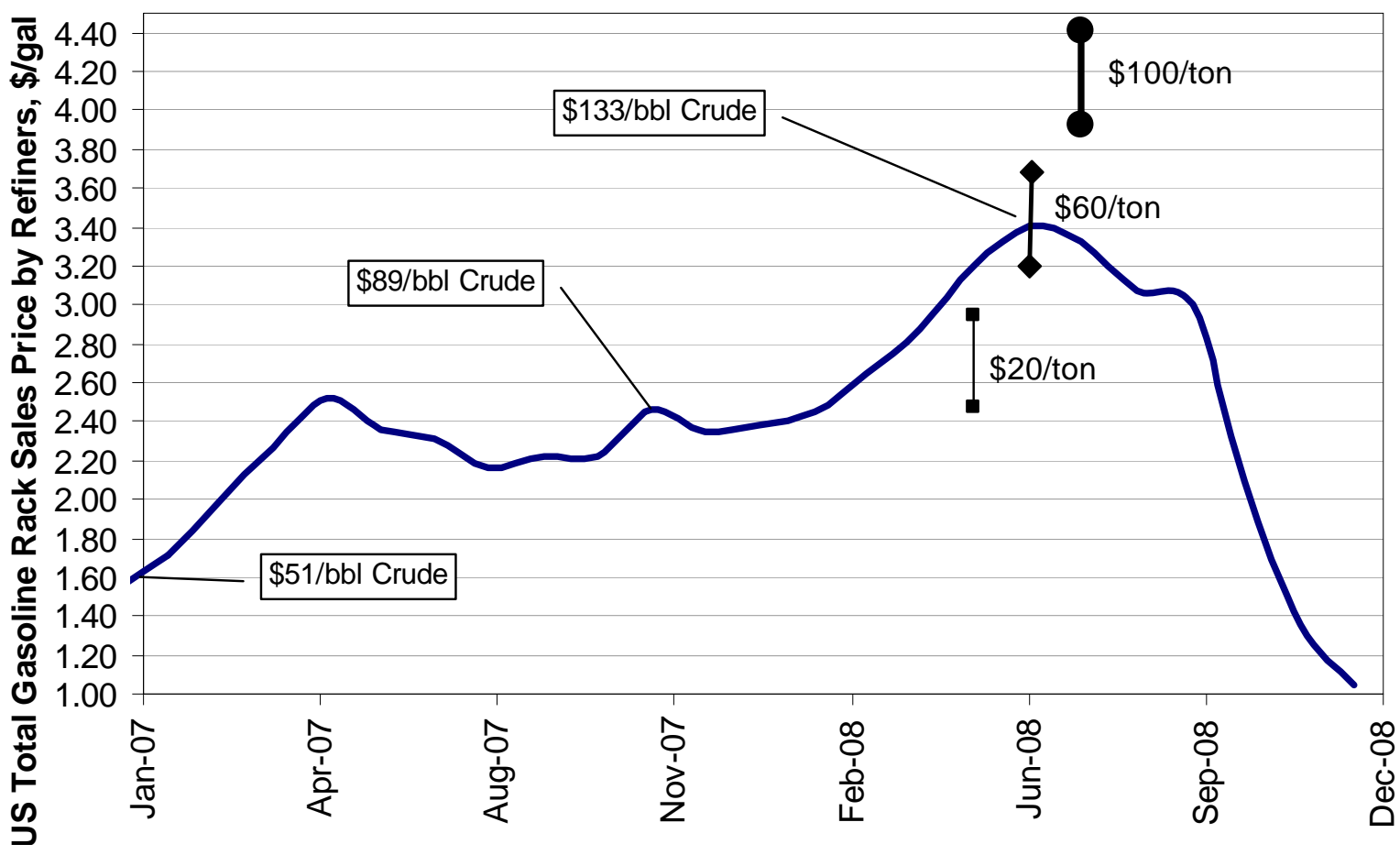

Figure 4-2 Effect of Feedstock Cost on Gasoline Price

\subsubsection{Sensitivity to Capital Cost and Yield}

There is scope for reducing the capital costs for the syngas cleanup and for the entire MTG synthesis process. The base case assumes that the gas cleanup step includes a tar reformer and a separate steam reformer. Additionally, the MTG process uses six fixed bed reactors:

- one for syngas to methanol synthesis,

- one for methanol to dimethyl ether (DME) conversion,

- $\quad$ three for methanol/DME conversion to gasoline and 
- one for hydrogenating the heavy gasoline fraction to remove durene.

Research into improved syngas cleanup is ongoing (Phillips, 2007). Pilot scale tests have shown that a continuous fluidized bed reactor can achieve nearly the same conversion to gasoline as the multi-fixed bed process (Cheng, 1994). MTG reactor design that avoid large recycle streams for temperature control, such as fluid bed MTG or shell and tube reactors, can reduce capital costs in the synthesis area. Additionally, combined synthesis steps, such as once through methanol/DME production could potentially reduce capital costs and product losses. This sensitivity explores the effect of capital cost reductions.

Figure 4-3 shows the producers selling price for three capital costs levels superimposed on the historic rack price data. The $100 \%$ bars are the base case, with the indirectly-heated gasifier the lower point and the directly-heated gasifier the upper. Reducing the capital to $75 \%$ of the base case brings the estimated rack price down by $\$ 0.40 /$ gallon. This corresponds to a crude price just above $\$ 100 / \mathrm{bbl}$. Further reducing the capital cost for the MTG synthesis step to $50 \%$ of the base case reduces the gasoline producers selling price by nearly $\$ 1 /$ gallon, but high crude costs are still needed. It appears that capital costs reduction alone may be insufficient to make this process viable.

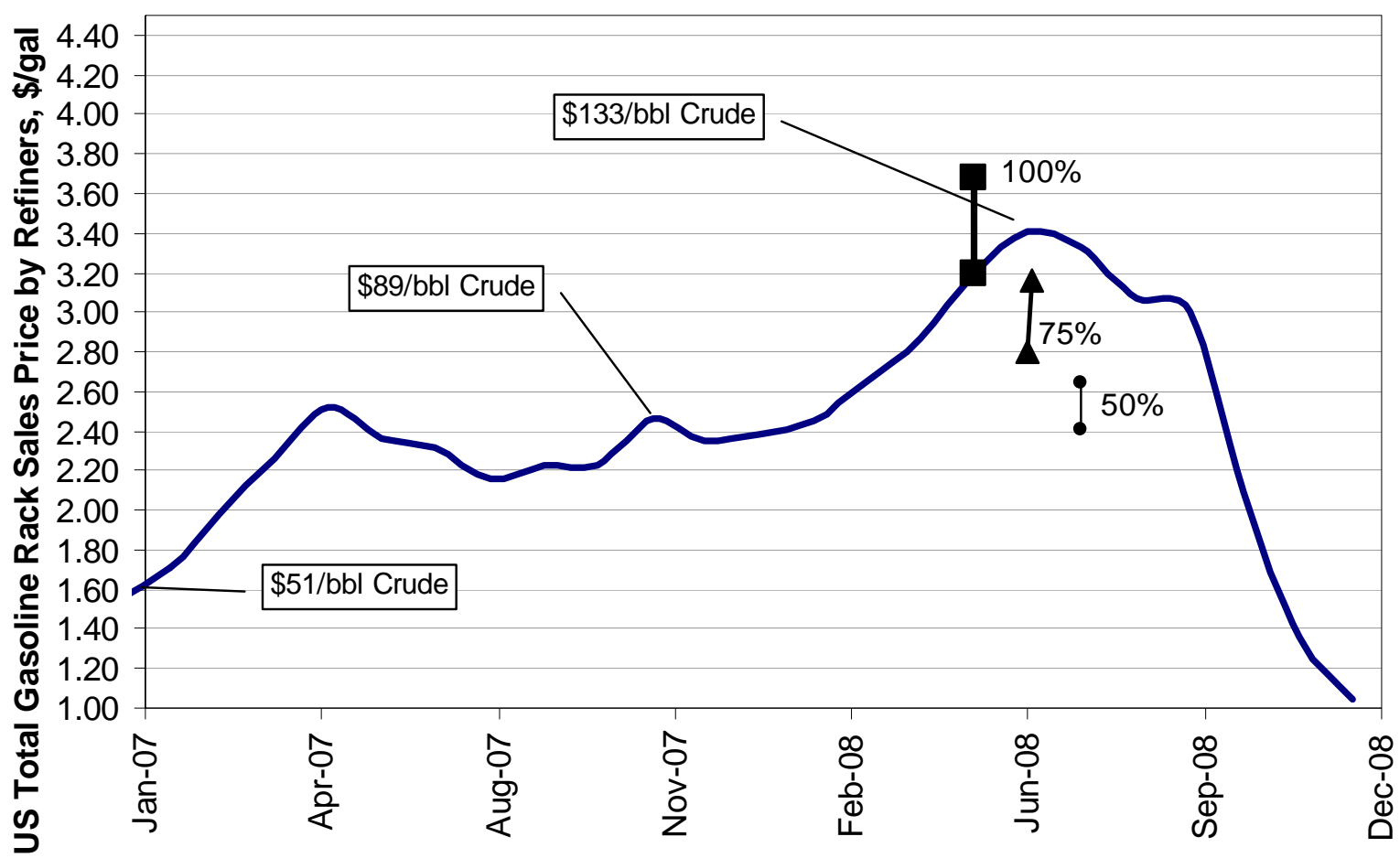

Figure 4-3 Effect of Capital Cost on Gasoline Price

Figure 4-4 shows the effect of reduced capital and improved yield. If all of the potential capital improvements are realized, and yield losses in the synthesis step are minimized, then the methanol-togasoline process estimated rack selling price can be lowered to approximately $\$ 2.00 /$ gallon and thus becomes economically attractive. 


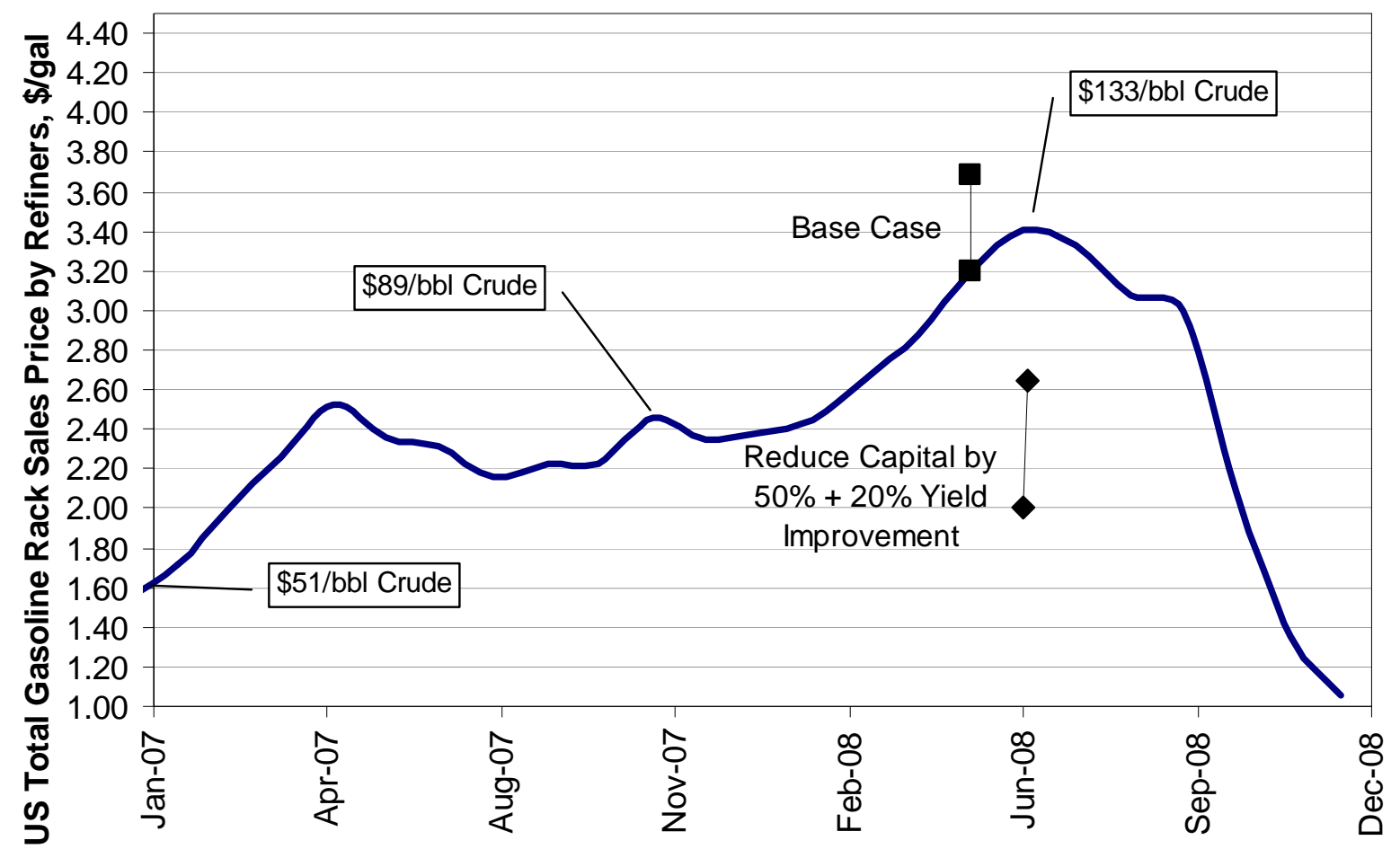

Figure 4-4 Effect of Capital Cost Reduction and Yield Improvement 


\subsection{Conclusions and Recommendations}

A techno-economic analysis (TEA) of a conventional biomass to gasoline process was conducted, based on existing technology. This process produces an infrastructure ready fuel, with a vast available market. The simulation results showed that the estimated gasoline selling prices for the indirectly-and directlyheated gasifier based systems result in rack selling prices higher than the average of the gasoline refiners rack price in the past year. The specific conclusions are:

1. The conventional MTG process is not commercially competitive at this scale unless crude prices are well above $\$ 100 / \mathrm{bbl}$.

2. The main capital costs areas are 1) gas cleanup steps (tar cracking, sulfur and other impurities removal and methane reduction by steam reforming) and 2) the MTG process, including methanol production, gas fractionation and HGT.

3. Sensitivity analysis indicated that:

a. Very low feedstock costs are required to make the fixed-bed MTG process economic.

b. Reducing the capital costs through consolidated gas cleanup and improved synthesis steps may significantly reduce the costs

c. Economic viability will likely require reduced capital and improved yield.

It is recommended that other processing alternatives should be simulated to identify the extent of process improvements. Such options should include:

- Consolidated gas cleanup,

- Alternative MTG reactor designs such as fluid-bed or shell \& tube,

- Consolidated synthesis with once through DME/Methanol reactors. 


\subsection{References}

Aden, A. 2008. Biochemical Production of Ethanol from corn Stover: 2007 State of Technology Model. NREL/TP-510-43205. National Renewable Energy Laboratory, Golden, CO. May 2008.

http://www.nrel.gov/docs/fy08osti/43205.pdf

Aden, A., P. Spath, and A. Atherton. 2005. The Potential of Thermochemical Ethanol Via Mixed Alcohols Production. Milestone Completion Report, FY05-684. National Renewable Energy Laboratory, Golden, CO. October 2005.

Chang, C.D., 1992. The New Zealand Gas-to-Gasoline Plant: An Engineering Tour de force. Catalysis Today, 13(1992):103-111.

Cheng, W., Kung, H. 1994. Methanol Production and Use. Marcel-Dekker. 1994.

DKRW website 2008. http://www.dkrwenergy.com/fw/main/Home-140.html.

Evans, R.J., R.A. Knight, M. Onischak, S.P. Babu, 1998. Development of Biomass Gasification to Produce Substitute Fuels. Institute of Gas Technology for Pacific Northwest Laboratory, PNL-6518, Richland, WA.

EIA, 2008a. Natural Gas Monthly. Energy Information Administration, Office of Oil and Gas, U.S. Department of energy, Washington, DC. http://www.eia.doe.gov/oil_gas/natural_gas/data_publications/natural_gas_monthly/ngm.html

EIA, 2008b. Electric Power Monthly - August 2008. DOE/EIA-0226. Energy Information Administration, Office of Coal, Nuclear, Electric and Alternative Fuels, U.S. Department of Energy, Washington, DC. http://www.eia.doe.gov/cneaf/electricity/epm/epm.pdf

EIA, 2009. Topics for Petroleum Prices. Energy Information Administration, Office of Oil and Gas, U.S. Department of energy, Washington, DC.

http://tonto.eia.doe.gov/dnav/pet/pet_pri top.asp

EISA 2007. "U.S. Energy Independence and Security Act of 2007," Public Law Number 110-140, signed 19 December, 2007; Title II. 2007. frwebgate.access.gpo.gov/cgi-

bin/getdoc.cgi?dbname=110_cong_bills\&docid=f:h6enr.txt.pdf

Fiedler, E., G. Grossmann, D. B. Kersebohm, G. Weiss, and C. Witte. 2000. Methanol. Ullmann's Encyclopedia of Industrial Chemistry, Electronic Release, 7th ed., Wiley-VCH, Weinheim, 2007.

Grace \& Co. 1982a. Preliminary Design and Assessment of a 50,000 BPD Coal-to-Gasoline-to-Methanol Plant. Process Engineering and Mechanical Design Reports Volume II of V. DOE/ET/14759-T1-Vol.214B, Prepared by W.R. Grace \&Co. for the U.S. Department of Energy, August 1982 
Grace \& Co. 1982b. Preliminary Design and Assessment of a 12,500 BPD Coal-to-Gasoline-to-Methanol Plant. Process Engineering and Mechanical Design Reports Volume II of IV. DOE/ET/14759-T3-Vol.214B, Prepared by W.R. Grace \&Co. for the U.S. Department of Energy, August 1982

Grace \& Co. 1982c. Preliminary Design and Assessment of a 12,500 BPD Coal-to-Gasoline-to-Methanol Plant. Capital and Operating Costs Estimates Volume I of IV. DOE/ET/14759-T3-Vol.1-18B, Prepared by W.R. Grace \&Co. for the U.S. Department of Energy, August 1982

Hamelinck, C.N., A.P.C. Faaij, 2001. Future prospects for production of methanol and hydrogen from biomass, NWS-E-2001-49, ISBN 90-73958-84-9, September 2001.

Hamelinck, C.N. and A.P.C. Faaij, 2002. Future Prospects for Production of Methanol and Hydrogen from Biomass. Journal of Power Sources, 111 (1):1-22. 18 September 2002.

Hamelinck, C.N., A.P.C. Faaij, H. den Uil, and H. Boerrigter. 2003. Production of FT Transportation Fuels from Biomass; Technical Options, Process Analysis and Optimization and Development Potential. NWS 90-393-3342-4. Utrecht University. The Netherlands, March 2003.

Kaneko, T., F. Derbyshire, E. Makino, D. Gray, and M. Tamura, 2002. Coal Liquefaction. Ullmann's Encyclopedia of Industrial Chemistry, Electronic Release, 7th ed., Wiley-VCH, Weinheim, 2007.

Ondry, G., 2008. Coal-to-Gasoline process will make U.S. debut. Chemical Engineering, 115(September 2008):16.

Phillips, S., A. Aden, J. Jechura, and D. Dayton, 2007. Thermochemical Ethanol via Indirect Gasification and Mixed Alcohol Synthesis of Lignocellulosic Biomass. NREL/TP-510-41168. April 2007.

Schuster, et al. 1985. Continuous Preparation of Ethanol. U.S. Patent 4,517,391. May 14, 1985.

Spath, P., A. Aden, T. Eggerman, M. Ringer, B. Wallace, J. Jechura, 2005. Biomass Hydrogen Production Detailed Design and Economics Utiltizing the Battelle Columbus Laboratory Indirectly Heated Gasifier. NREL/TP-510-37408. National Renewable Energy Laboratory, Golden, CO.

SRI PEP 2003 Yearbook International. SRI Consulting, United States. Menlo Park, CA. 2003.

SRI PEP 2007 Yearbook International. SRI Consulting, United States. Menlo Park, CA. 2007.

Tabak, S., X. Zhao, A. Brandl, and M. Heinritz-Adrian, 2008. An Alternative Route for Coal to Liquid Fuel - ExxonMobil Methanol-to-gasoline (MTG) Process. First World Coal-to-Liquids Conference, April 3-4, 2008, Paris, France. 
Appendix A. Heat and Material Balances for the Indirectlyheated Gasifier Case 


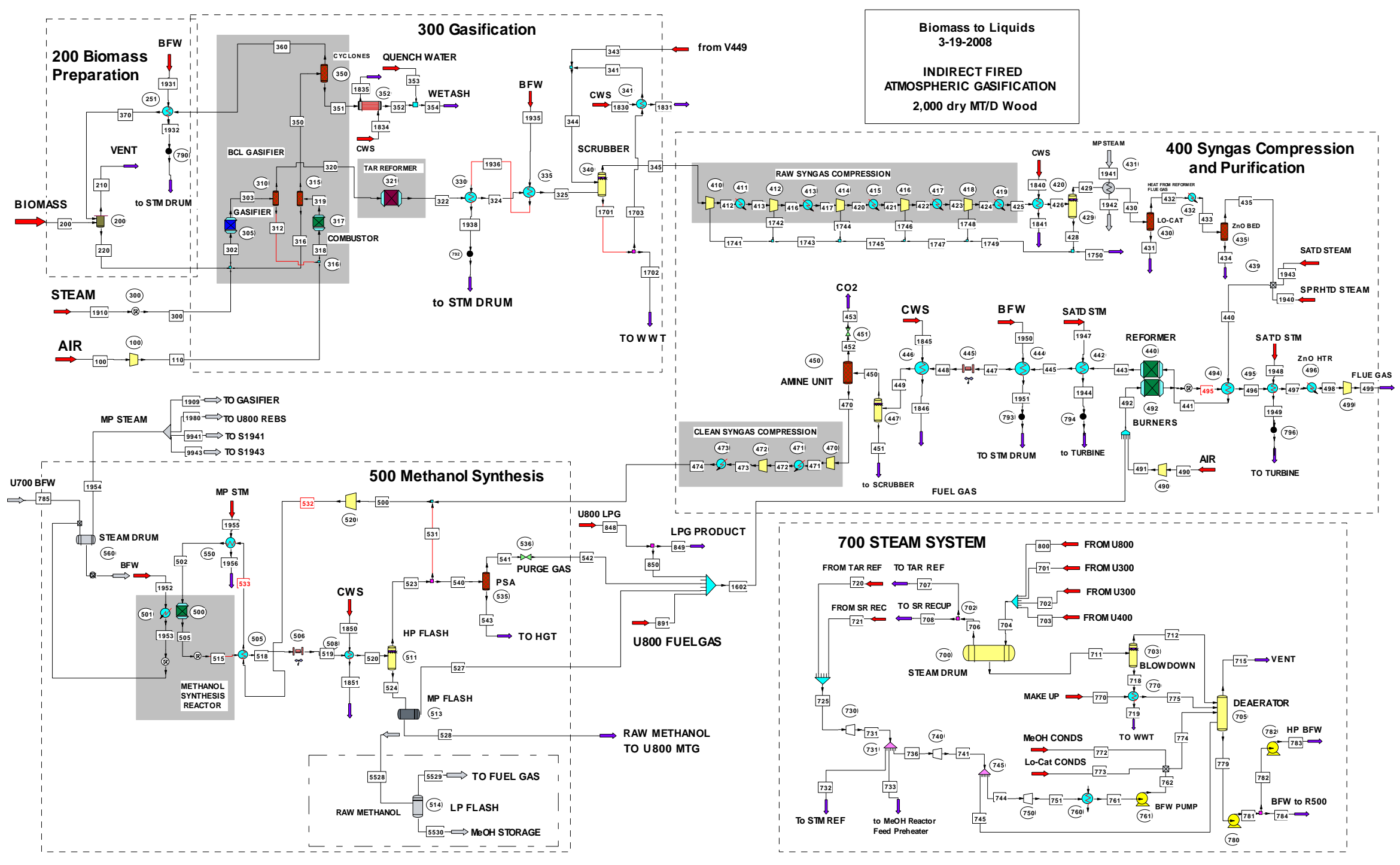

Figure A-1 Process Flow Diagram for the Indirectly-heated Gasifier Case - Feed Prep through Methanol Synthesis 
Table A-1 Steam Results for the Indirectly-heated Gasifier Case - Feed Prep through Methanol Synthesis

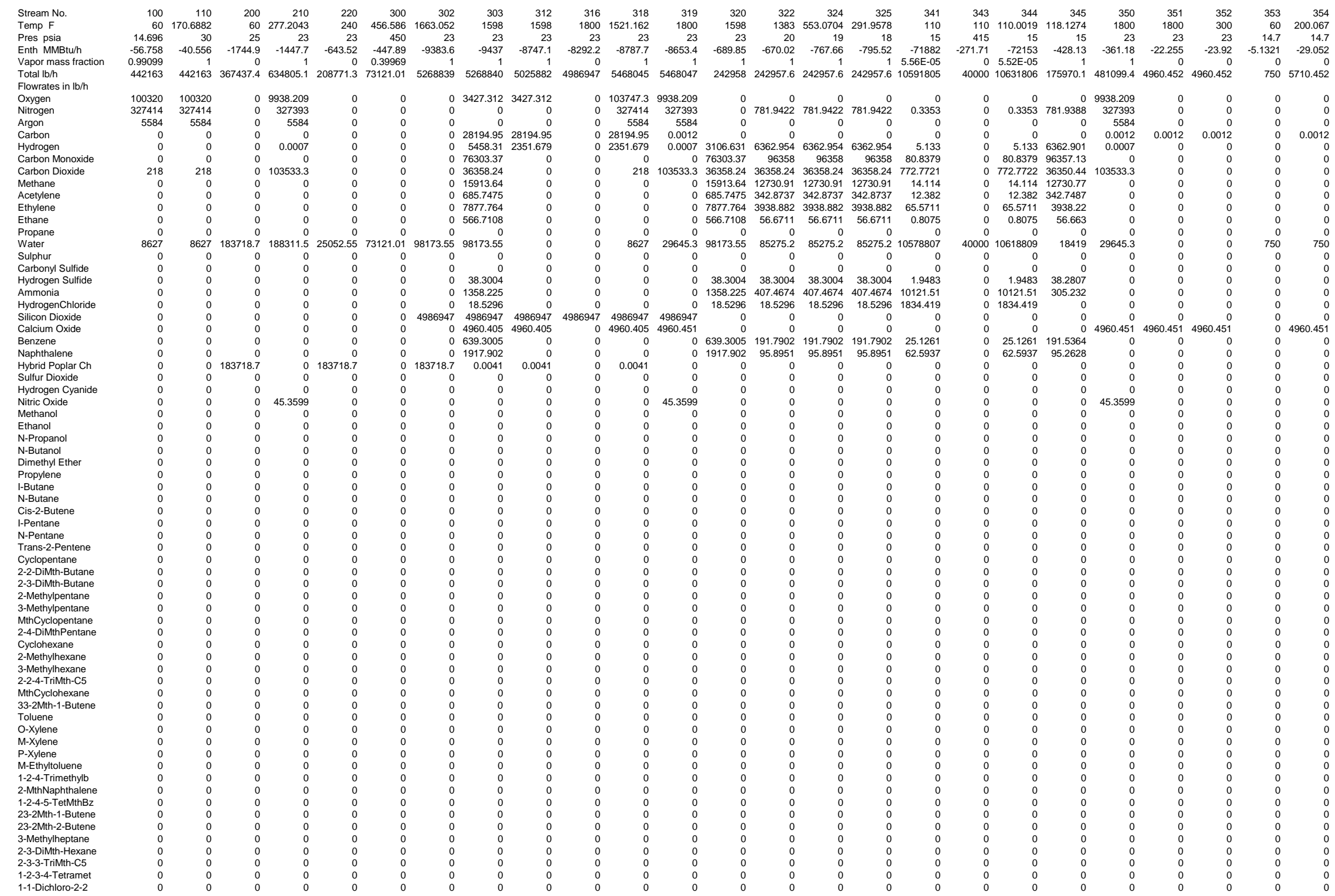




\begin{tabular}{|c|c|c|c|c|c|c|c|c|c|c|c|c|c|c|c|c|c|c|c|c|c|c|c|c|c|}
\hline Stream No. & & 370 & & 413 & & 417 & 420 & 421 & 422 & 423 & & 425 & 426 & 428 & 429 & 430 & 431 & 432 & 433 & 434 & 435 & 440 & 441 & 443 & \\
\hline Temp F & 1800 & 1750 & 252.8497 & 140 & 268.9654 & 140 & 194.8721 & 140 & 207.8941 & 140 & $\begin{array}{ll}0 & 229.1196 \\
\end{array}$ & 140 & 110 & 110 & 110 & 120 & 120 & 120 & 707 & 707 & 707 & $07 \quad 711.727$ & 955 & 1652 & 1353.795 \\
\hline $\begin{array}{l}\text { Pres psia } \\
\text { Pnth }\end{array}$ & 23 & 23 & 28 & $\begin{array}{l}8 \\
6\end{array} 28$ & 54 & 54 & 109.5 & $\begin{array}{r}109.5 \\
1273\end{array}$ & $\begin{array}{r}220 \\
2757\end{array}$ & 220 & $\begin{array}{l}0 \\
0\end{array} \quad 465$ & $\begin{array}{r}465 \\
5\end{array}$ & 465 & $\begin{array}{r}465 \\
\end{array}$ & 465 & 460 & 460 & $\begin{array}{r}460 \\
42508\end{array}$ & $\begin{array}{r}460 \\
8652\end{array}$ & 460 & 460 & $\begin{array}{l}60 \\
65\end{array}$ & 445 & $\begin{array}{r}430 \\
8730\end{array}$ & 427.5 \\
\hline $\begin{array}{l}\text { Enth MMBtsulh } \\
\text { Vapor mass fraction }\end{array}$ & $\begin{aligned}-338.92 \\
1\end{aligned}$ & & $\begin{array}{r}-418.46 \\
1\end{array}$ & 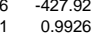 & & $\begin{array}{l}-4366.67 \\
0.94363\end{array}$ & $\begin{aligned}-408.89 \\
1\end{aligned}$ & $\begin{array}{l}-424.73 \\
0.93177\end{array}$ & $\begin{array}{r}-375.7 \\
1\end{array}$ & $\begin{array}{l}-388.67 \\
0.95098\end{array}$ & & $\begin{array}{r}-371.83 \\
0.9606\end{array}$ & $\begin{array}{l}3 \\
\begin{array}{l}3 \\
6\end{array} \\
6\end{array}$ & & & $\begin{array}{r}-3.25 .08 \\
0.9992 \\
-1\end{array}$ & & $\begin{array}{r}-325.58 \\
0.9992 \\
0\end{array}$ & & & & & & $\begin{array}{l}-873.13 \\
1 \\
1\end{array}$ & $\begin{array}{r}-921.85 \\
1\end{array}$ \\
\hline Total lb/h & 476139 & 476139 & 175970.1 & $\begin{array}{ll}1 & 175970.1\end{array}$ & 175970.1 & $\begin{array}{l}175970.1 \\
\end{array}$ & 173546 & 173546 & 167893 & 167893 & $\begin{array}{ll}3 & 165215.7\end{array}$ & $\begin{array}{l}0.90215 .7 \\
1652\end{array}$ & $\begin{array}{lll}0 & 0.95039 \\
7 & 165215.7\end{array}$ & 7204.441 & 158011.2 & 158011.2 & & $\begin{array}{l}158011.2 \\
15892\end{array}$ & $2 \quad 158011.2$ & & 12157671.7 & $\begin{array}{l}.7 \\
.7\end{array} 298516$ & 298516 & 298517 & 298517 \\
\hline $\begin{array}{l}\text { Flowrates in lb/h } \\
\text { Oxygen }\end{array}$ & 9938.209 & 9938.209 & & & & & & & & & & & & & & & & & & & & & & & \\
\hline trogen & & 327393 & 781.9388 & $\begin{array}{ll}8 & 781.9388\end{array}$ & 781.9388 & 781.9388 & 781.9388 & 781.9388 & 781.9388 & 781.9388 & $\begin{array}{ll}8 & 781.9388\end{array}$ & 781.9388 & 8781.9388 & & 781.9387 & 781.9387 & & 781.9387 & 781.9387 & & & $87 \quad 781.9387$ & 781.9387 & 767.1981 & 767.1981 \\
\hline Argon & 5584 & 5584 & & & & & & & & & & & & 0 & & & 0 & & & & 0 & & & & \\
\hline Carbon & 0 & 0 & & & & & & & & & & & & 0 & & & 0 & & 0 & & 0 & 0 & 0 & 0 & \\
\hline Hydrogen & 0.0007 & 0.0007 & 6362.901 & 16362.901 & 6362.901 & 6362.901 & $\begin{array}{r}6362.9 \\
\end{array}$ & $\begin{array}{r}6362.9 \\
\end{array}$ & 6362.898 & 6362.898 & $\begin{array}{ll}8 & 6362.893 \\
1\end{array}$ & 6362.893 & $\begin{array}{l}36362.893 \\
6\end{array}$ & 0.0017 & 6362.891 & 6362.891 & & 6362.891 & $1 \quad 6362.891$ & & $0 \quad 6362.891$ & $91 \quad 6362.891$ & 6362.891 & 12914.3 & 12914.3 \\
\hline Carbon Monoxide & 0 & 0 & 96357.13 & 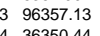 & 96357.13 & 96357.13 & 96357.12 & 96357.12 & $\begin{array}{r}96357.1 \\
63677\end{array}$ & 96357.1 & 196357.06 & 96357.06 & $\begin{array}{ll}696357.06 \\
6\end{array}$ & $\begin{array}{l}0.0114 \\
180200\end{array}$ & 96357.05 & 96357.05 & 0 & 96357.05 & 596357.05 & & $\begin{array}{ll}0 & 96357.05 \\
0 & 3625\end{array}$ & $\begin{array}{ll}05 & 96357.05 \\
20\end{array}$ & 96357.05 & 82509.18 & 82509.18 \\
\hline $\begin{array}{l}\text { Carbon Dioxide } \\
\text { Methane }\end{array}$ & $\begin{array}{r}103533.3 \\
0\end{array}$ & & $\begin{array}{l}36350.44 \\
1270.77\end{array}$ & 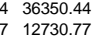 & $\begin{array}{l}36350.44 \\
12730.77\end{array}$ & $\begin{array}{l}36350.44 \\
12730.77\end{array}$ & $\begin{array}{l}36349.75 \\
172730.77\end{array}$ & $\begin{array}{l}36349.75 \\
172730.77\end{array}$ & $\begin{array}{l}36376.77 \\
12730.75\end{array}$ & $\begin{array}{l}36346.77 \\
12730.75\end{array}$ & $\begin{array}{ll}7 & 36344.16 \\
5 & 12730.72\end{array}$ & $\begin{array}{l}36344.16 \\
12730.72\end{array}$ & $\begin{array}{l}636344.16 \\
2127072 \\
1270\end{array}$ & $\begin{array}{r}18.9229 \\
0.162\end{array}$ & $\begin{array}{l}36325.23 \\
12730.71\end{array}$ & $\begin{array}{l}36325.23 \\
1270.71\end{array}$ & $\begin{array}{l}0 \\
0\end{array}$ & $\begin{array}{l}36325.23 \\
17230.71\end{array}$ & $\begin{array}{l}3 \quad 36325.23 \\
1 \\
1 \\
1270.71\end{array}$ & & $\begin{array}{ll}0 & 36325.23 \\
0 & 1273071\end{array}$ & $\begin{array}{l}23 \\
23 \\
71\end{array}$ & $\begin{array}{l}36325.23 \\
1273071\end{array}$ & $\begin{array}{l}92337.79 \\
5568085\end{array}$ & $\begin{array}{l}92337.79 \\
5586085\end{array}$ \\
\hline $\begin{array}{l}\text { ethane } \\
\text { cetylene }\end{array}$ & $\begin{array}{l}0 \\
0\end{array}$ & & $\begin{array}{l}1272.1 / 1 \\
342.7487\end{array}$ & 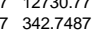 & 342.7487 & $\begin{array}{l}12730 . / 11 \\
342.7487\end{array}$ & $\begin{array}{l}17230 . / 17 \\
342.7475\end{array}$ & $\begin{array}{l}123.30 .17 \\
342.7475\end{array}$ & $\begin{array}{r}1230.15 \\
342.741\end{array}$ & $\begin{array}{r}127 / 70.15 \\
342.741\end{array}$ & $\begin{array}{l}5 \\
1 \\
1\end{array}$ & $\begin{array}{r}12 / 70.72 \\
342.734 \\
4\end{array}$ & $\begin{array}{lll}2 & 127 / 70 . / 2 \\
4 & 342.734\end{array}$ & $\begin{array}{l}.0 .0162 \\
0.0094\end{array}$ & $\begin{array}{l}11730 . / 1 \\
342.7245\end{array}$ & $\begin{array}{l}12 / 70.71 \\
342.7245\end{array}$ & & $\begin{array}{l}12 / 30 . / 1 \\
342.7245\end{array}$ & 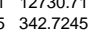 & & $\begin{array}{lll}0 & 112 / 30 . / 1 \\
0 & 342.7245\end{array}$ & $\begin{array}{lll}71 & 12730.11 \\
45 & 3427245\end{array}$ & 12730.71 & 5586.085 & . 5586.085 \\
\hline hylene & & & $\begin{array}{r}342.1487 \\
3938.22\end{array}$ & $\begin{array}{l}2 \\
2 \\
2\end{array}$ & 3938.22 & (3938.22 & 3938.217 & $\begin{array}{l}3938.217 \\
3938\end{array}$ & 3983.201 & 3938.201 & $\begin{array}{ll}1 \\
1 \\
39383.18\end{array}$ & 3998.18 & $\begin{array}{ll}8 \\
8\end{array}$ & 0.0124 & $\begin{array}{l}392.1245 \\
3938.168\end{array}$ & 3938.168 & & 398.168 & $\begin{array}{l}8 \\
3 \\
3938.868\end{array}$ & & $\begin{array}{l}0 \\
0\end{array} 3938.168$ & $\begin{array}{l}68 \\
68 \\
39388.168\end{array}$ & 398.168 & 0.1222 & 0.1222 \\
\hline hane & 0 & & 年66.63 & $3 \quad 56663$ & 56.663 & 56.663 & 56.6629 & 56.6629 & 56.6627 & 56.6627 & $7 \quad 56.6625$ & 56.6625 & $\begin{array}{ll}5 & 56.6625\end{array}$ & 0.001 & 56.6623 & 56.6623 & & 5 & $\begin{array}{ll}3 & 56.6623\end{array}$ & & 66.6623 & 6623 & 6623 & 0.3203 & 0.3203 \\
\hline $\begin{array}{l}\text { Propane } \\
\text { Pron }\end{array}$ & & & $\begin{array}{l}3.000 \\
0\end{array}$ & 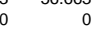 & -50.000 & $\begin{array}{r}0.000 \\
0\end{array}$ & $\begin{array}{r}30.0029 \\
0\end{array}$ & & $\begin{array}{r}00.0021 \\
0\end{array}$ & $\begin{array}{l}0.0021 \\
0\end{array}$ & $\begin{array}{l}0 \\
0\end{array}$ & b & & & & (5.0020 & & $\begin{aligned} 0.0020 \\
0\end{aligned}$ & 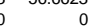 & & .0020 & 0 & & & \\
\hline Water & 29645.3 & 29645.3 & 18419 & 18419 & 18419 & 18419 & 15995.75 & 15995.75 & 10346.32 & 10346.32 & $2 \quad 7672.177$ & 7672.177 & 77672.177 & 7182.581 & 489.5941 & 489.5941 & & 489.5941 & 1489.5941 & & 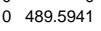 & 1333.6 & 33.6 & 104384 & 104384 \\
\hline ulphur & & 0 & 0 & 0 & 0 & 0 & 0 & & 0 & 0 & 0 & 0 & 0 & 0 & 0 & 0 & 0 & 0 & 0 & 0 & 0 & 0 & 0 & 0 & \\
\hline $\begin{array}{l}\text { Carbonyl Sulfide } \\
\text { Hydrogen Sulfide }\end{array}$ & & & $\begin{array}{r}0 \\
38.2807\end{array}$ & $\begin{array}{lr}0 & 0 \\
7 & 38.2807\end{array}$ & $\begin{array}{r}0 \\
38.2807\end{array}$ & $\begin{array}{r}0 \\
38.2807\end{array}$ & $\begin{array}{r}0 \\
38.2774\end{array}$ & $\begin{array}{r}0 \\
382774\end{array}$ & $\begin{array}{r}0 \\
382615\end{array}$ & $\begin{array}{r}0 \\
38.2615\end{array}$ & $\begin{array}{lr}0 & 0 \\
5 & 38246\end{array}$ & $\begin{array}{r}0 \\
38246\end{array}$ & $\begin{array}{l}0 \\
0 \\
6\end{array}$ & $\begin{array}{r}0 \\
0.0442\end{array}$ & $\begin{aligned} 0 \\
382017\end{aligned}$ & $\begin{array}{r}0 \\
382017\end{array}$ & & $\begin{array}{r}0 \\
38.2017\end{array}$ & $\begin{array}{r}0 \\
0 \\
7 \\
782017\end{array}$ & $\begin{array}{r}0 \\
382017\end{array}$ & & $\begin{array}{l}0 \\
0\end{array}$ & $\begin{array}{l}0 \\
0\end{array}$ & $\begin{array}{l}0 \\
0\end{array}$ & \\
\hline Ammonia & & & 305.232 & $2 \quad 305.232$ & 305.232 & 305.232 & 305.1141 & 305.1141 & $\begin{array}{r}304.5999 \\
3\end{array}$ & $\begin{array}{l}304.5999 \\
3049\end{array}$ & $\begin{array}{l}0 \\
9 \\
9\end{array}$ & $\begin{array}{r}304.1574 \\
+4\end{array}$ & $\begin{array}{l}0 \\
4\end{array}$ & 2.832 & 301.3254 & 301.3254 & & 301.3254 & $\begin{array}{l}4 \quad 301.3254 \\
4\end{array}$ & 301.3254 & & 0 & 0 & 17.9266 & 17.9266 \\
\hline Hydrogenchloride & & & 0 & 年 0 & 0 & & 0 & 0 & 0 & 0 & $\begin{array}{ll}0 & 0\end{array}$ & 0 & $0 \quad 0$ & 0 & 0 & 0 & 0 & 0 & & 0 & 0 & 0 & & & \\
\hline $\begin{array}{l}\text { Silicon Dioxide } \\
\text { Paride }\end{array}$ & & & & 0 & & & & & 0 & 0 & 0 & & 0 & 0 & 0 & 0 & 0 & 0 & 0 & & 0 & 0 & 0 & 0 & \\
\hline $\begin{array}{l}\text { alcium Oxide } \\
\text { enzzene }\end{array}$ & & & $\begin{array}{r}0 \\
191.5364\end{array}$ & $\begin{array}{rr}0 & 0 \\
4 & 191.5364\end{array}$ & $\begin{array}{r}0 \\
191.5364\end{array}$ & $\begin{array}{r}0 \\
191.5364\end{array}$ & $\begin{array}{r}0 \\
191.5341\end{array}$ & $\begin{array}{r}0 \\
191.5341\end{array}$ & $\begin{array}{r}0 \\
191.5221\end{array}$ & $\begin{array}{r}0 \\
191.5221\end{array}$ & $\begin{array}{lr}0 & 0 \\
1 & 191.5079\end{array}$ & $\begin{array}{r}0 \\
191.5079\end{array}$ & $\begin{array}{l}0 \\
0 \\
9\end{array}$ & $\begin{array}{r}0 \\
0.0074\end{array}$ & $\begin{array}{r}0 \\
191.5005\end{array}$ & $\begin{array}{r}0 \\
191.5005\end{array}$ & $\begin{array}{l}0 \\
0\end{array}$ & $\begin{array}{r}0 \\
191.5005\end{array}$ & 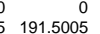 & & 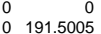 & 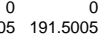 & $\begin{array}{r}0 \\
5 \\
5\end{array}$ & $\begin{array}{l}0 \\
0\end{array}$ & $\begin{array}{l}0 \\
0 \\
0\end{array}$ \\
\hline Naphthalene & & & 95.2628 & $\begin{array}{ll}8 & 95.2628\end{array}$ & 95.2628 & 95.2628 & 95.2614 & 95.2614 & 95.2532 & 95.2532 & $2 \quad 95.243$ & 95.243 & $3 \quad 95.243$ & 0.0022 & 95.2408 & 95.2408 & & 95.2408 & $\begin{array}{ll} & 95.2408\end{array}$ & & $\begin{array}{l}0 \quad 95.2408 \\
0\end{array}$ & $\begin{array}{l}08 \quad 95.2408 \\
08\end{array}$ & $3 \quad 95.2408$ & 0 & \\
\hline ybbrid Poplar Ch & 0 & & & & & & & & 0 & 0 & 0 & & 0 & 0 & 0 & & 0 & 0 & 0 & 0 & 0 & $\begin{array}{lll}0 & 0\end{array}$ & & 0 & 0 \\
\hline Ulfur Dioxide & & 0 & 0 & 0 & 0 & 0 & 0 & 0 & 0 & ${ }_{0}^{0}$ & $0_{0}^{0}$ & 0 & 0 & 0 & 0 & 0 & 0 & 0 & 0 & & 0 & 0 & 0 & 0 & 0 \\
\hline $\begin{array}{l}\text { yorogen Cyanide } \\
\text { itric Oxide }\end{array}$ & 45.3599 & 45.3599 & 0 & 0 & $\begin{array}{l}0 \\
0\end{array}$ & 0 & 0 & $\begin{array}{l}0 \\
0\end{array}$ & 0 & 0 & 0 & & 0 & 0 & 0 & 0 & 0 & 0 & $\begin{array}{l}0 \\
0\end{array}$ & & 0 & $\begin{array}{l}0 \\
0 \\
0\end{array}$ & & 0 & $\begin{array}{l}0 \\
0 \\
0\end{array}$ \\
\hline nethanol & 0 & 0 & 0 & 0 & 0 & 0 & 0 & 0 & 0 & 0 & 0 & & 0 & 0 & 0 & 0 & 0 & 0 & 0 & & 0 & 0 & 0 & 0.056 & 0.056 \\
\hline thanol & 0 & 0 & 0 & 0 & 0 & 0 & 0 & 0 & 0 & 0 & 0 & 0 & 0 & 0 & 0 & 0 & 0 & 0 & 0 & & 0 & 0 & 0 & 0 & 0 \\
\hline $\begin{array}{l}\text { - Propanal } \\
\text {-Butanol }\end{array}$ & $\begin{array}{l}0 \\
0\end{array}$ & $\begin{array}{c}0 \\
0\end{array}$ & & $\begin{array}{l}0 \\
0\end{array}$ & $\begin{array}{l}0 \\
0\end{array}$ & $\begin{array}{l}0 \\
0\end{array}$ & $\begin{array}{l}0 \\
0\end{array}$ & $\begin{array}{l}0 \\
0\end{array}$ & $\begin{array}{l}0 \\
0\end{array}$ & $\begin{array}{l}0 \\
0 \\
0\end{array}$ & $\begin{array}{l}0 \\
0\end{array}$ & & $\begin{array}{l}0 \\
0\end{array}$ & $\begin{array}{l}0 \\
0\end{array}$ & $\begin{array}{l}0 \\
0\end{array}$ & $\begin{array}{l}0 \\
0\end{array}$ & $\begin{array}{l}0 \\
0\end{array}$ & $\begin{array}{l}0 \\
0\end{array}$ & $\begin{array}{l}0 \\
0\end{array}$ & & $\begin{array}{l}0 \\
0 \\
0\end{array}$ & $\begin{array}{l}0 \\
0\end{array}$ & & $\begin{array}{l}0 \\
0\end{array}$ & $\begin{array}{l}0 \\
0\end{array}$ \\
\hline imethyl Ether & 0 & 0 & 0 & 0 & 0 & 0 & 0 & 0 & 0 & 0 & 0 & 0 & 0 & 0 & 0 & 0 & 0 & 0 & 0 & & 0 & 0 & 0 & 0 & 0 \\
\hline opylene & 0 & 0 & 0 & 0 & 0 & 0 & 0 & 0 & 0 & 0 & 0 & 0 & 0 & 0 & 0 & 0 & 0 & 0 & 0 & & 0 & 0 & 0 & 0 & 0 \\
\hline Butane & 0 & 0 & 0 & 0 & 0 & 0 & 0 & 0 & 0 & 0 & 0 & 0 & 0 & 0 & 0 & 0 & 0 & 0 & 0 & & 0 & 0 & & 0 & 0 \\
\hline -Butane & $0_{0}^{0}$ & $\begin{array}{c}0 \\
0 \\
0\end{array}$ & $\begin{array}{l}0 \\
0 \\
0\end{array}$ & 0 & ${ }^{0}$ & $0_{0}^{0}$ & 0 & 0 & 0 & $0_{0}^{0}$ & $0_{0}^{0}$ & 0 & 0 & 0 & 0 & 0 & 0 & 0 & 0 & & 0 & 0 & 0 & 0 & 0 \\
\hline s-2-Butene & 0 & $0_{0}^{0}-2$ & 0 & 0 & ${ }_{0}^{0}$ & 0 & 0 & 0 & 0 & 0 & 0 & 0 & 0 & 0 & 0 & 0 & 0 & 0 & 0 & & 0 & 0 & 0 & 0 & 0 \\
\hline $\begin{array}{l}\text { Pentane } \\
\text { Pentane }\end{array}$ & 0 & 0 & 0 & 0 & 0 & $\begin{array}{l}0 \\
0\end{array}$ & 0 & 0 & 0 & 0 & 0 & 0 & 0 & 0 & 0 & 0 & 0 & 0 & $\begin{array}{l}0 \\
0\end{array}$ & & 0 & 0 & 0 & $\begin{array}{l}0 \\
0\end{array}$ & 0 \\
\hline rans-2-Pentene & 0 & 0 & 0 & 0 & 0 & 0 & 0 & 0 & 0 & 0 & 0 & & 0 & 0 & 0 & 0 & 0 & 0 & 0 & & 0 & & & & \\
\hline Cyclopentane & 0 & 0 & 0 & 0 & 0 & 0 & 0 & 0 & 0 & 0 & 0 & & 0 & 0 & 0 & 0 & 0 & 0 & 0 & & 0 & 0 & 0 & 0 & 0 \\
\hline 2-2-DiMth-Butane & 0 & 0 & 0 & 0 & 0 & 0 & 0 & 0 & 0 & 0 & 0 & & 0 & 0 & 0 & 0 & 0 & 0 & 0 & 0 & 0 & 0 & 0 & 0 & 0 \\
\hline ane & 0 & 0 & 0 & 0 & 0 & & 0 & & 0 & 0 & 0 & 0 & 0 & 0 & 0 & 0 & 0 & 0 & 0 & 0 & 0 & 0 & 0 & 0 & 0 \\
\hline thylpentane & $\begin{array}{l}0 \\
0\end{array}$ & $\begin{array}{l}0 \\
0 \\
0\end{array}$ & $\begin{array}{l}0 \\
0 \\
0\end{array}$ & 0 & 0 & $\begin{array}{l}0 \\
0\end{array}$ & $\begin{array}{l}0 \\
0\end{array}$ & 0 & $\begin{array}{l}0 \\
0\end{array}$ & 0 & $\begin{array}{l}0 \\
0\end{array}$ & & $\begin{array}{l}0 \\
0 \\
0\end{array}$ & $\begin{array}{l}0 \\
0\end{array}$ & $\begin{array}{l}0 \\
0\end{array}$ & $\begin{array}{l}0 \\
0 \\
0\end{array}$ & $\begin{array}{l}0 \\
0\end{array}$ & & $\begin{array}{l}0 \\
0\end{array}$ & & $\begin{array}{l}0 \\
0\end{array}$ & 0 & & $\begin{array}{l}0 \\
0\end{array}$ & $\begin{array}{l}0 \\
0 \\
0\end{array}$ \\
\hline thCyclopentane & 0 & 0 & 0 & 0 & 0 & 0 & 0 & 0 & 0 & 0 & 0 & & 0 & 0 & 0 & 0 & 0 & 0 & 0 & 0 & 0 & 0 & 0 & 0 & 0 \\
\hline & 0 & 0 & 0 & 0 & 0 & 0 & 0 & 0 & 0 & 0 & 0 & 0 & 0 & 0 & 0 & 0 & 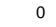 & 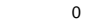 & 0 & 0 & 0 & 0 & 0 & 0 & 0 \\
\hline nexane & 0 & 0 & 0 & 0 & 0 & 0 & 0 & 0 & 0 & 0 & 0 & 0 & 0 & 0 & 0 & 0 & 0 & 列 & 0 & 0 & 0 & 0 & 0 & 0 & 0 \\
\hline $\begin{array}{l}\text { hylhexane } \\
\text { hylhexane }\end{array}$ & $\begin{array}{l}0 \\
0 \\
0\end{array}$ & $\begin{array}{c}0 \\
0 \\
0\end{array}$ & $\begin{array}{l}0 \\
0 \\
0\end{array}$ & $\begin{array}{l}0 \\
0 \\
0\end{array}$ & $\begin{array}{l}0 \\
0\end{array}$ & $\begin{array}{l}0 \\
0\end{array}$ & $\begin{array}{l}0 \\
0\end{array}$ & $\begin{array}{l}0 \\
0 \\
0\end{array}$ & 0 & $\begin{array}{l}0 \\
0 \\
0\end{array}$ & $\begin{array}{l}0 \\
0 \\
0\end{array}$ & $\begin{array}{l}0 \\
0 \\
0\end{array}$ & $\begin{array}{l}0 \\
0 \\
0\end{array}$ & 0 & 0 & 0 & 0 & 0 & 0 & 0 & 0 & 0 & 0 & 0 & $\begin{array}{l}0 \\
0 \\
0\end{array}$ \\
\hline $\begin{array}{l}\text { ylhexane } \\
\text { yrimth-c5 }\end{array}$ & 0 & 0 & 0 & $\begin{array}{l}0 \\
0\end{array}$ & $\begin{array}{l}0 \\
0\end{array}$ & $\begin{array}{l}0 \\
0\end{array}$ & $\begin{array}{l}0 \\
0\end{array}$ & 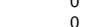 & $\begin{array}{l}0 \\
0\end{array}$ & o & $\begin{array}{l}0 \\
0\end{array}$ & & $\begin{array}{l}0 \\
0 \\
0\end{array}$ & $\begin{array}{l}0 \\
0\end{array}$ & $\begin{array}{l}0 \\
0\end{array}$ & 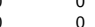 & 0 & 0 & 0 & 0 & 0 & 0 & 0 & 0 & $\begin{array}{l}0 \\
0 \\
0\end{array}$ \\
\hline Cane & 0 & 0 & 0 & 0 & 0 & 0 & 0 & 0 & 0 & 0 & 0 & & 0 & 0 & 0 & & 0 & 0 & 0 & & 0 & 0 & 0 & 0 & 0 \\
\hline 33-2Mth-1-Butene & 0 & 0 & 0 & 0 & 0 & 0 & & & 0 & 0 & 0 & & 0 & 0 & 0 & & 0 & & 0 & & 0 & 0 & & 0 & 0 \\
\hline Tolue & 0 & 0 & 0 & 0 & 0 & 0 & 0 & 0 & 0 & 0 & 0 & & 0 & 0 & 0 & & 0 & 0 & 0 & & 0 & 0 & 0 & 0 & 0 \\
\hline D-Xylene & 0 & 0 & 0 & 0 & 0 & 0 & 0 & 0 & 0 & 0 & 0 & & 0 & 0 & 0 & 0 & 0 & 0 & 0 & 0 & 0 & 0 & 0 & 0 & 0 \\
\hline - & 0 & 0 & 0 & 0 & 0 & 0 & 0 & 0 & 0 & 0 & 0 & & 0 & 0 & 0 & 0 & 0 & 0 & 0 & 0 & 0 & 0 & 0 & 0 & 0 \\
\hline yet & $\begin{array}{l}0 \\
0\end{array}$ & $\begin{array}{l}0 \\
0 \\
0\end{array}$ & $\begin{array}{l}0 \\
0 \\
0\end{array}$ & $\begin{array}{l}0 \\
0\end{array}$ & $\begin{array}{l}0 \\
0\end{array}$ & $\begin{array}{l}0 \\
0\end{array}$ & $\begin{array}{l}0 \\
0\end{array}$ & $\begin{array}{l}0 \\
0 \\
0\end{array}$ & $\begin{array}{l}0 \\
0\end{array}$ & $\begin{array}{l}0 \\
0\end{array}$ & $\begin{array}{l}0 \\
0\end{array}$ & & $\begin{array}{l}0 \\
0\end{array}$ & $\begin{array}{l}0 \\
0\end{array}$ & $\begin{array}{l}0 \\
0\end{array}$ & & 0 & $\begin{array}{l}0 \\
0\end{array}$ & $\begin{array}{l}0 \\
0\end{array}$ & $\begin{array}{l}0 \\
0 \\
0\end{array}$ & $\begin{array}{l}0 \\
0 \\
0\end{array}$ & $\begin{array}{l}0 \\
0 \\
0\end{array}$ & $\begin{array}{l}0 \\
0 \\
0\end{array}$ & $\begin{array}{l}0 \\
0\end{array}$ & $\begin{array}{l}0 \\
0 \\
0\end{array}$ \\
\hline nethylb & 0 & 0 & 0 & 0 & 0 & 0 & 0 & 0 & 0 & 0 & 0 & & 0 & 0 & 0 & 0 & 0 & 0 & 0 & 0 & 0 & 0 & 0 & 0 & 0 \\
\hline & & & & 0 & 0 & & 0 & & 0 & 0 & 0 & & 0 & 0 & 0 & & 0 & & 0 & & 0 & 0 & & 0 & \\
\hline & 0 & 0 & 0 & 0 & 0 & 0 & 0 & 0 & 0 & 0 & 0 & & 0 & 0 & 0 & & 0 & 0 & 0 & & 0 & 0 & 0 & 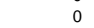 & \\
\hline & 0 & 0 & 0 & 0 & 0 & 0 & 0 & 0 & 0 & 0 & 0 & & 0 & 0 & 0 & & 0 & & 0 & & 0 & & & & 0 \\
\hline 23-2Mth-2-Butene & 0 & 0 & 0 & 0 & 0 & 0 & 0 & 0 & 0 & 0 & 0 & 0 & 0 & 0 & 0 & & 0 & 0 & 0 & 0 & 0 & 0 & 0 & 0 & 0 \\
\hline neptane & 0 & 0 & 0 & 0 & 0 & 0 & 0 & 0 & 0 & 0 & 0 & & 0 & 0 & 0 & & 0 & 0 & 0 & & 0 & 0 & 0 & 0 & 0 \\
\hline 3-DiMth-Hexane & 0 & 0 & 0 & 0 & 0 & 0 & 0 & 0 & 0 & 0 & 0 & & 0 & 0 & 0 & 0 & 0 & 0 & 0 & & 0 & 0 & 0 & 0 & 0 \\
\hline -3-4-Tetramet & $\begin{array}{l}0 \\
0\end{array}$ & $\begin{array}{l}0 \\
0\end{array}$ & $\begin{array}{l}0 \\
0\end{array}$ & $\begin{array}{l}0 \\
0\end{array}$ & $\begin{array}{l}0 \\
0\end{array}$ & 0 & $\begin{array}{l}0 \\
0\end{array}$ & 0 & $\begin{array}{l}0 \\
0\end{array}$ & $\begin{array}{l}0 \\
0\end{array}$ & $\begin{array}{l}0 \\
0\end{array}$ & & $\begin{array}{l}0 \\
0\end{array}$ & $\begin{array}{l}0 \\
0\end{array}$ & 0 & 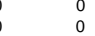 & & $\begin{array}{l}0 \\
0\end{array}$ & $\begin{array}{l}0 \\
0\end{array}$ & & $\begin{array}{l}0 \\
0\end{array}$ & 0 & & & 0 \\
\hline 1-1-Dichloro-2-2 & 0 & 0 & 0 & 0 & 0 & & 0 & 0 & 0 & 0 & 0 & & 0 & 0 & & & & & & & 0 & 0 & & & \\
\hline
\end{tabular}




\begin{tabular}{|c|c|c|c|c|c|c|c|c|c|c|c|c|c|c|c|c|c|c|c|c|c|c|c|c|c|}
\hline Stream No. & 447 & 448 & 449 & 450 & 451 & 452 & 453 & 470 & 471 & 472 & 473 & 474 & 490 & 491 & 492 & 495 & 496 & 497 & 498 & 499 & 500 & 502 & 505 & 515 & 518 \\
\hline Temp F & 300 & 150 & 110 & 110 & 110 & 120 & 59.7881 & 120 & 205.2048 & 150 & 242.3097 & 150 & $\begin{array}{l}490 \\
60\end{array}$ & 124.4883 & 111.1885 & 1800 & 1367.633 & 870.1641 & 316.4648 & 334.2016 & 106.6531 & 446 & 500 & 500 & $\begin{array}{l}190.0061 \\
0.061\end{array}$ \\
\hline Pres psia & 427.5 & 427.5 & 425 & 425 & 425 & 420 & 22 & 420 & 614.6959 & 614.6959 & 914.6959 & 914.6959 & 14.696 & 20 & 20 & 20 & 15 & 15 & 15 & 16 & 820 & 890 & 840 & 840 & 835 \\
\hline th MMBtu/h & -1136.8 & -1203 & -1212.1 & -506.27 & -705.86 & -322.85 & -322.85 & & -177.47 & -181.43 & & & -0.98453 & 2.6915 & -38.497 & -188.33 & -223.66 & -262.14 & -301.94 & -300.71 & -1589.5 & -1408.1 & -1499 & -1499 & 1666.2 \\
\hline $\begin{array}{l}\text { Vapor mass fraction } \\
\text { Total b/h }\end{array}$ & 0.79311 & 0.65634 & 0.65126 & 1 & $\begin{array}{r}0 \\
0\end{array}$ & $\begin{array}{r}0.99343 \\
\end{array}$ & $\begin{array}{r}0.99586 \\
\end{array}$ & 1 & 1 & 1 & 1 & 1 & 1 & 1 & $\frac{1}{1}$ & 1 & 11 & 1 & 1 & 1 & 1 & 1 & $\begin{array}{r}1 \\
0.643 \\
0\end{array}$ & 1 & 0.98888 \\
\hline Flowrates in Ib/h & & & & & & & & & & & & & & & & & & & & & & & & & 846420 \\
\hline $\begin{array}{l}\text { ygen } \\
\text { rogaen }\end{array}$ & $\begin{array}{r}0 \\
7671981\end{array}$ & 0 & $\begin{array}{r}0 \\
7\end{array}$ & $\begin{array}{r}0 \\
7671977\end{array}$ & 0 & 0 & 0 & 1077 & $\begin{array}{r}0 \\
7671077\end{array}$ & 0 & & & 54861.66 & 54861.66 & 54861.66 & 6746.636 & 6745.959 & 6745.959 & 6745.959 & 6745.959 & & & & & \\
\hline $\begin{array}{l}\text { rigogen } \\
\text { rgon }\end{array}$ & & & & & & $\begin{array}{l}0 \\
0\end{array}$ & & & & & & & & & & & $\begin{array}{l}181436.1 \\
0\end{array}$ & $\begin{array}{r}181436.1 \\
0\end{array}$ & $\begin{array}{r}181436.1 \\
0\end{array}$ & $\begin{array}{r}181436.1 \\
0\end{array}$ & & 37731.27 0 & & & \\
\hline rbon & & & & & 0 & 0 & 0 & 0 & & & & & 0 & 0 & & & & & & & 0 & 0 & & & \\
\hline (1) & 12914.3 & 12914.3 & 12914.3 & 12914.27 & 0.0315 & 0 & & 12914.27 & 12914.27 & & 12914.27 & 12914.27 & 0 & & 1042.396 & 0.0007 & 0.0007 & 0.0007 & 0.0007 & 0.0007 & 65847.7 & 65847.7 & 54030.01 & 54030.01 & 54027.54 \\
\hline rbon Monoxide & 82509.18 & 82509.18 & $\begin{array}{l}82509.18 \\
0237\end{array}$ & 82509.09 & 0.0913 & & & 82509.09 & 82509.09 & 82509.09 & 82509.09 & 82509.09 & 0 & 0 & 5629.909 & 0.009 & 0.009 & 0.009 & 0.009 & 0.009 & 354816.5 & 354816.5 & 277987 & 277987 & 277981.1 \\
\hline $\begin{array}{l}\text { ribon Dioxide } \\
\text { thane }\end{array}$ & $\begin{array}{l}92337.79 \\
5586.085\end{array}$ & $\begin{array}{r}92337.79 \\
5566.085\end{array}$ & $\begin{array}{l}92337.79 \\
5586.085\end{array}$ & $\begin{array}{l}91884.29 \\
5586.019\end{array}$ & $\begin{array}{r}453.507 \\
0.0669\end{array}$ & $\begin{array}{r}82695.86 \\
0\end{array}$ & 82695.86 & $\begin{array}{l}9188.431 \\
558019\end{array}$ & $\begin{array}{l}9188.431 \\
5586019\end{array}$ & $\begin{array}{l}9188.431 \\
5586.019\end{array}$ & $\begin{array}{l}9188.431 \\
5586019\end{array}$ & $\begin{array}{l}9188.431 \\
5586.019\end{array}$ & $\begin{array}{l}0 \\
0 \\
0\end{array}$ & $\begin{array}{l}0 \\
0\end{array}$ & $\begin{array}{l}4036.357 \\
5640355\end{array}$ & $\begin{array}{r}40361.23 \\
0.0001\end{array}$ & $\begin{array}{r}40362.66 \\
00001\end{array}$ & $\begin{array}{r}40362.66 \\
0.0001\end{array}$ & 40362.66 & 40362.66 & 113173.3 & $\begin{array}{l}113173.3 \\
2509091\end{array}$ & 107647.8 & 107647.8 & $\begin{array}{l}107619.3 \\
2589825\end{array}$ \\
\hline etylene & & $\begin{array}{l}500.0005 \\
0.0005\end{array}$ & $\begin{array}{l}5000.0005 \\
0.0005\end{array}$ & $\begin{array}{l}500005 \\
0.0005\end{array}$ & & 0 & 0 & $\begin{array}{l}50.0005 \\
0.005\end{array}$ & 0.0005 & $\begin{array}{l}0.0005 \\
0.019\end{array}$ & 0.0005 & 0.0005 & 0 & 0 & 0 & 0.0002 & 0.002 & - & 0.002 & 0.002 & - & $\begin{array}{l}258996.1 \\
0.0131\end{array}$ & 年 & 0.0131 & $\begin{array}{l}258982.5 \\
0.131\end{array}$ \\
\hline $\begin{array}{l}\text { elylene } \\
\text { yylene }\end{array}$ & 0.1222 & 0.1222 & 0.1222 & 0.1222 & 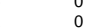 & 0 & 0 & 0.1222 & 0.1222 & 0.1222 & 0.1222 & $\begin{array}{l}0.0005 \\
0.1222\end{array}$ & 0 & 0 & 1012 & 0.0002 & 0.0002 & 0.0002 & 0.0002 & 0.0002 & 46027 & 46027 & 46027 & 46027 & 0.0131 \\
\hline lane & 0.3203 & 0.3203 & 0.3203 & 0.3203 & 0 & & & 0.3203 & 0.3203 & 0.3203 & 0.3203 & 0.3203 & 0 & & 31.9187 & 0.0003 & 0.0003 & & 0.0003 & 0.0003 & 120623 & 12.0623 & 12.0623 & 120623 & $\begin{array}{r}4.0021 \\
120605\end{array}$ \\
\hline ropane & 0 & (2) & 0 & 0 & & 0 & 0 & & & 0 & $\begin{array}{l}0.5200 \\
0\end{array}$ & 0 & 0 & & 923.1663 & 0.0004 & 0.0004 & 0.0004 & 0.0004 & 0.0004 & $\begin{array}{l}12.0020 \\
0\end{array}$ & $\begin{array}{l}12.0005 \\
0\end{array}$ & $\begin{array}{ll}12.0020 \\
0\end{array}$ & $\begin{aligned} 12.0020 \\
0\end{aligned}$ & \\
\hline Nater & 104384 & 104384 & 104384 & 735.557 & 103648.5 & 735.557 & 735.5569 & 0 & 0 & 0 & 0 & 0 & 0 & 0 & 18.7381 & 28245.16 & 28245.33 & 28245.33 & 28245.33 & 28245.33 & 87.6452 & 87.6452 & 2349.449 & 2349.449 & 2348.768 \\
\hline phur & & & & & & 0 & 0 & 0 & 0 & 0 & 0 & 0 & 0 & 0 & 0 & 0 & 0 & & 0 & & & 0 & 0 & 0 & \\
\hline $\begin{array}{l}\text { bronyl Sulfide } \\
\text { drogen Sulfide }\end{array}$ & & & & & $\begin{array}{l}0 \\
0\end{array}$ & $\begin{array}{l}0 \\
0 \\
0\end{array}$ & $\begin{array}{l}0 \\
0 \\
0\end{array}$ & $\begin{array}{l}0 \\
0 \\
0\end{array}$ & $\begin{array}{l}0 \\
0\end{array}$ & $\begin{array}{l}0 \\
0 \\
0\end{array}$ & 0 & $\begin{array}{l}0 \\
0\end{array}$ & $\begin{array}{l}0 \\
0 \\
0\end{array}$ & $\begin{array}{l}0 \\
0\end{array}$ & 0 & 0 & 0 & & 0 & 0 & 0 & 0 & 0 & 0 & \\
\hline $\begin{array}{l}\text { darogens } \\
\text { nmonia }\end{array}$ & 17.9266 & 17.9266 & 17.9266 & 16.453 & 1.4736 & 0 & $\begin{array}{l}0 \\
0\end{array}$ & 16.453 & 16.453 & 16.453 & 16.453 & 16.453 & 0 & 0 & $\begin{array}{l}1.7605 \\
\end{array}$ & 0.0002 & 0.0002 & 0.0002 & 0.0002 & 0.0002 & 100.6533 & 100.6533 & 100.6533 & 100.6533 & $\begin{array}{r}0 \\
100.6477\end{array}$ \\
\hline drogenChloride & 0 & & & 0 & 0 & 0 & 0 & 0 & 0 & 0 & 0 & 0 & 0 & 0 & 0 & 0 & 0 & 0 & 0 & 0 & 0 & 0 & 0 & 0 & \\
\hline Acium & & & 0 & 0 & 0 & 0 & 0 & 0 & 0 & 0 & 0 & 0 & 0 & 0 & 0 & 0 & 0 & 0 & 0 & & & & & & 0 \\
\hline $\begin{array}{l}\text { Calclum } \\
\text { Benzene }\end{array}$ & 0 & & $\begin{array}{l}0 \\
0\end{array}$ & 0 & $\begin{array}{l}0 \\
0\end{array}$ & $\begin{array}{l}0 \\
0 \\
0\end{array}$ & $\begin{array}{l}0 \\
0 \\
0\end{array}$ & $\begin{array}{l}0 \\
0 \\
0\end{array}$ & $\begin{array}{l}0 \\
0\end{array}$ & $\begin{array}{l}0 \\
0\end{array}$ & $\begin{array}{l}0 \\
0\end{array}$ & $\begin{array}{l}0 \\
0\end{array}$ & $\begin{array}{l}0 \\
0\end{array}$ & $\begin{array}{l}0 \\
0\end{array}$ & 0.1397 & 0.0006 & $\begin{array}{r}0 \\
0.0006\end{array}$ & $\begin{array}{r}0 \\
0.0006\end{array}$ & $\begin{array}{r}0 \\
0.0006\end{array}$ & $\begin{array}{r}0 \\
0.0006\end{array}$ & $\begin{array}{l}0 \\
0\end{array}$ & $\begin{array}{l}0 \\
0\end{array}$ & $\begin{array}{l}0 \\
0 \\
0\end{array}$ & $\begin{array}{l}0 \\
0\end{array}$ & $\begin{array}{l}0 \\
0 \\
0\end{array}$ \\
\hline iphthalene & & & & 0 & & 0 & 0 & 0 & 0 & 0 & 0 & 0 & 0 & 0 & 0.1495 & 0.0007 & 0.0007 & 0.0007 & 0.0007 & 0.0007 & 0 & 0 & 0 & 0 & \\
\hline ybrid Poplar Ch & 0 & & 0 & 0 & 0 & 0 & 0 & 0 & 0 & 0 & 0 & 0 & 0 & 0 & 0 & 0 & 0 & & 0 & 0 & 0 & 0 & 0 & 0 & 0 \\
\hline $\begin{array}{l}\text { fur Dioxide } \\
\text { rogogen Cyanid }\end{array}$ & & & 0 & 0 & 0 & 0 & ${ }_{0}^{0}$ & 0 & 0 & 0 & 0 & 0 & 0 & 0 & 0 & & 0 & 0 & 0 & 0 & 0 & 0 & 0 & 0 & \\
\hline Jitric Oxide & 0 & 0 & $\begin{array}{l}0 \\
0\end{array}$ & $\begin{array}{l}0 \\
0\end{array}$ & & $\begin{array}{l}0 \\
0 \\
0\end{array}$ & $\begin{array}{l}0 \\
0\end{array}$ & $\begin{array}{l}0 \\
0\end{array}$ & $\begin{array}{l}0 \\
0\end{array}$ & $\begin{array}{l}0 \\
0\end{array}$ & $\begin{array}{l}0 \\
0\end{array}$ & $\begin{array}{l}0 \\
0\end{array}$ & $\begin{array}{l}0 \\
0\end{array}$ & $\begin{array}{l}0 \\
0\end{array}$ & $\begin{array}{l}0 \\
0\end{array}$ & $\begin{array}{r}0 \\
27.825\end{array}$ & $\begin{array}{r}0 \\
27.8236\end{array}$ & $\begin{array}{r}0 \\
27.8236\end{array}$ & $\begin{array}{r}0 \\
27.8236\end{array}$ & $\begin{array}{r}0 \\
27.8236\end{array}$ & 0 & & & $\begin{array}{l}0 \\
0\end{array}$ & \\
\hline${ }^{2}=0$ & 0.056 & 0.056 & 0.056 & 0.0038 & 0.0521 & 0 & 0 & 0.0038 & 0.0038 & 0.0038 & 0.0038 & 0.0038 & 0 & & 327.7415 & 0.0003 & 0.0003 & 0.0003 & 0.0003 & 0.0003 & 157 & & & & \\
\hline thanol & 0 & 0 & 0 & & 0 & 0 & 0 & 0 & 0 & 0 & 0 & 0 & 0 & 0 & 0 & & & & & 0 & & & & & \\
\hline $\begin{array}{l}\text { Propanol } \\
\text { Butanol }\end{array}$ & $\begin{array}{l}0 \\
0\end{array}$ & 0 & $\begin{array}{l}0 \\
0\end{array}$ & $\begin{array}{l}0 \\
0\end{array}$ & 0 & $\begin{array}{l}0 \\
0 \\
0\end{array}$ & ${ }_{0}^{0}$ & 0 & ${ }_{0}^{0}$ & ${ }_{0}^{0}$ & 0 & 0 & 0 & 0 & 0 & 0 & 0 & 0 & 0 & 0 & 0 & 0 & 0 & 0 & 0 \\
\hline Ether & 0 & 0 & 0 & 0 & 0 & 0 & 0 & 0 & 0 & 0 & 0 & 0 & 0 & 0 & & & & & & & & & & 0 & $\begin{array}{l}0 \\
0 \\
0\end{array}$ \\
\hline povlene & 0 & 0 & 0 & 0 & 0 & 0 & 0 & 0 & 0 & 0 & 0 & 0 & 0 & 0 & 10,657 & 00003 & 0.0003 & 00003 & 0.0003 & 0.0003 & 0 & 0 & 0 & 0 & $\begin{array}{l}0 \\
0 \\
0\end{array}$ \\
\hline utan & 0 & 0 & 0 & 0 & 0 & 0 & 0 & 0 & 0 & 0 & 0 & 0 & 0 & 0 & 1533.925 & 04 & & & & & 0 & & 0 & 0 & 0 \\
\hline & 0 & 0 & 0 & 0 & 0 & 0 & 0 & 0 & 0 & 0 & 0 & 0 & 0 & & 461. & 0.0004 & & & & & 0 & 0 & 0 & 0 & 0 \\
\hline s-2-Butene & 0 & 0 & 0 & 0 & 0 & 0 & 0 & 0 & 0 & 0 & 0 & 0 & 0 & & 259.5576 & 0.0004 & 0.0004 & 04 & & 0.0004 & 0 & 0 & 0 & 0 & 0 \\
\hline nonts & 0 & 0 & 0 & 0 & 0 & 0 & 0 & 0 & 0 & 0 & 0 & 0 & 0 & & 467 & & & & & & 0 & 0 & 0 & 0 & 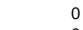 \\
\hline & 0 & 0 & 0 & 0 & 0 & 0 & 0 & 0 & 0 & 0 & 0 & 0 & 0 & 0 & & & & & & & & 0 & & 0 & 0 \\
\hline yclope & 0 & 0 & 0 & 0 & 0 & 0 & 0 & 0 & $\begin{array}{l}0 \\
0\end{array}$ & $\begin{array}{l}0 \\
0\end{array}$ & 0 & 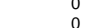 & 0 & $\begin{array}{l}0 \\
0\end{array}$ & $\begin{array}{c}5 / .2 \\
0.2\end{array}$ & & & & & & & 0 & 0 & 0 & 0 \\
\hline & 0 & 0 & 0 & 0 & 0 & 0 & & & 0 & & 0 & & & 0 & & & & & & & & & 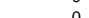 & 0 & 0 \\
\hline & 0 & 0 & 0 & 0 & 0 & 0 & 0 & 0 & 0 & 0 & 0 & 0 & 0 & 0 & 3.0 & & & & & & 0 & 0 & 0 & 0 & 0 \\
\hline & 0 & 0 & 0 & 0 & 0 & 0 & 0 & 0 & 0 & 0 & 0 & 0 & 0 & 0 & 2.5339 & & & & & & & 0 & 0 & 0 & 0 \\
\hline & 0 & 0 & 0 & 0 & 0 & 0 & 0 & 0 & 0 & 0 & 0 & 0 & 0 & 0 & 1.2 & & & & & & 0 & 0 & 0 & 0 & 0 \\
\hline e & 0 & 0 & 0 & 0 & 0 & 0 & 0 & 0 & 0 & 0 & 0 & 0 & 0 & 0 & 0.25 & & 66 & & & 00 & 0 & 0 & 0 & 0 & 0 \\
\hline & 0 & 0 & 0 & 0 & 0 & 0 & 0 & 0 & 0 & a & 0 & & 0 & 0 & & & & & & & & 0 & 0 & 0 & 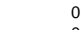 \\
\hline & 0 & 0 & 0 & 0 & 0 & 0 & 0 & 0 & 0 & & 0 & & & 0 & & & & & & & & 0 & 0 & 0 & \\
\hline & 0 & 0 & 0 & 0 & 0 & 0 & 0 & 0 & 0 & 0 & 0 & & & 0 & & & & & & & & & ( & 0 & 0 \\
\hline & 0 & 0 & 0 & 0 & 5 & 0 & & 0 & 0 & & & & & 0 & & & & & & & & & 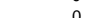 & 0 & 0 \\
\hline & 0 & 0 & 0 & 0 & 0 & 0 & 0 & 0 & 0 & & & & 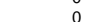 & 0 & 0 & & & & & & & & 0 & 0 & 0 \\
\hline & 0 & $c$ & 0 & 0 & 0 & 0 & 0 & & 0 & & $c$ & & $c$ & 0 & & & & & & & & & 0 & 0 & 0 \\
\hline Toluene & 0 & 0 & 0 & 0 & 0 & 0 & 0 & 0 & 0 & a & 0 & & 0 & 0 & & & & & & & & 0 & 0 & 0 & 0 \\
\hline & & 0 & 0 & 0 & 0 & & 0 & 0 & 0 & & & & & 0 & & & & & & & & & 0 & 0 & 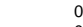 \\
\hline & 0 & 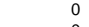 & 0 & 0 & 0 & 0 & 0 & 0 & 0 & 0 & 0 & & 0 & 0 & & & & & & & & 0 & 0 & 0 & 0 \\
\hline & 0 & $\begin{array}{l}0 \\
0\end{array}$ & $\begin{array}{l}0 \\
0\end{array}$ & $\begin{array}{l}0 \\
0\end{array}$ & $\begin{array}{l}0 \\
0\end{array}$ & 0 & $\begin{array}{l}0 \\
0 \\
0\end{array}$ & $\begin{array}{l}0 \\
0 \\
0\end{array}$ & $\begin{array}{l}0 \\
0 \\
0\end{array}$ & $\begin{array}{l}0 \\
0 \\
0\end{array}$ & $\begin{array}{l}0 \\
0\end{array}$ & $\begin{array}{l}0 \\
0 \\
0\end{array}$ & $\begin{array}{l}0 \\
0 \\
0\end{array}$ & $\begin{array}{l}0 \\
0 \\
0\end{array}$ & 0. & & 0.0007 & & 0.0007 & 000 & & 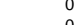 & 0 & 0 & 0 \\
\hline & 0 & 0 & 0 & 0 & 0 & 0 & 0 & 0 & 0 & 0 & 0 & & 0 & 0 & & & & & 0 & & & & & 0 & 0 \\
\hline & 0 & 0 & 0 & 0 & 0 & 0 & 0 & 0 & 0 & 0 & 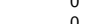 & & 0 & 0 & & 8 & & & 08 & & 0 & 0 & 0 & 0 & $\begin{array}{l}0 \\
0 \\
0\end{array}$ \\
\hline & 0 & 0 & 0 & 0 & 0 & 0 & 0 & 0 & c & & 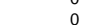 & & 0 & 0 & 0.00 & & & & & & & 0 & 0 & 0 & 0 \\
\hline & 0 & 0 & 0 & 0 & 0 & 0 & 0 & c & 0 & & 0 & & 0 & 0 & 1.0061 & 0.00 & & & & & 0 & 0 & 0 & 0 & 0 \\
\hline & 0 & 0 & 0 & 0 & 0 & 0 & 0 & 0 & 0 & 0 & c & & 0 & 0 & 0. & & & & & & 0 & 0 & 0 & 0 & 0 \\
\hline & 0 & & & & 0 & 0 & 0 & 0 & 0 & & & & 0 & . & & & & & & & U & 0 & 0 & 0 & 0 \\
\hline & 0 & 0 & 0 & 0 & 0 & 0 & 0 & 0 & 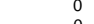 & 0 & 0 & & 0 & 0 & & & & & & & 0 & 0 & 0 & 0 & 0 \\
\hline & 0 & & 0 & 0 & 0 & 0 & $\begin{array}{l}0 \\
0 \\
0\end{array}$ & 0 & 0 & 0 & 0 & & $\begin{array}{l}0 \\
0 \\
0\end{array}$ & 0 & 1.2974 & $\begin{array}{l}0.00066 \\
0.0006\end{array}$ & $\begin{array}{l}0.00006 \\
0.0006\end{array}$ & & $\begin{array}{l}0.0006 \\
0.0006\end{array}$ & $\begin{array}{l}0.0006 \\
0.0006\end{array}$ & & & $\begin{array}{l}0 \\
0\end{array}$ & $\begin{array}{l}0 \\
0 \\
0\end{array}$ & \\
\hline oro-2-2 & 0 & 0 & 0 & 0 & 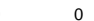 & 0 & 0 & 0 & 0 & & 0 & & & 0 & & & & & & & & & 0 & 0 & \\
\hline
\end{tabular}




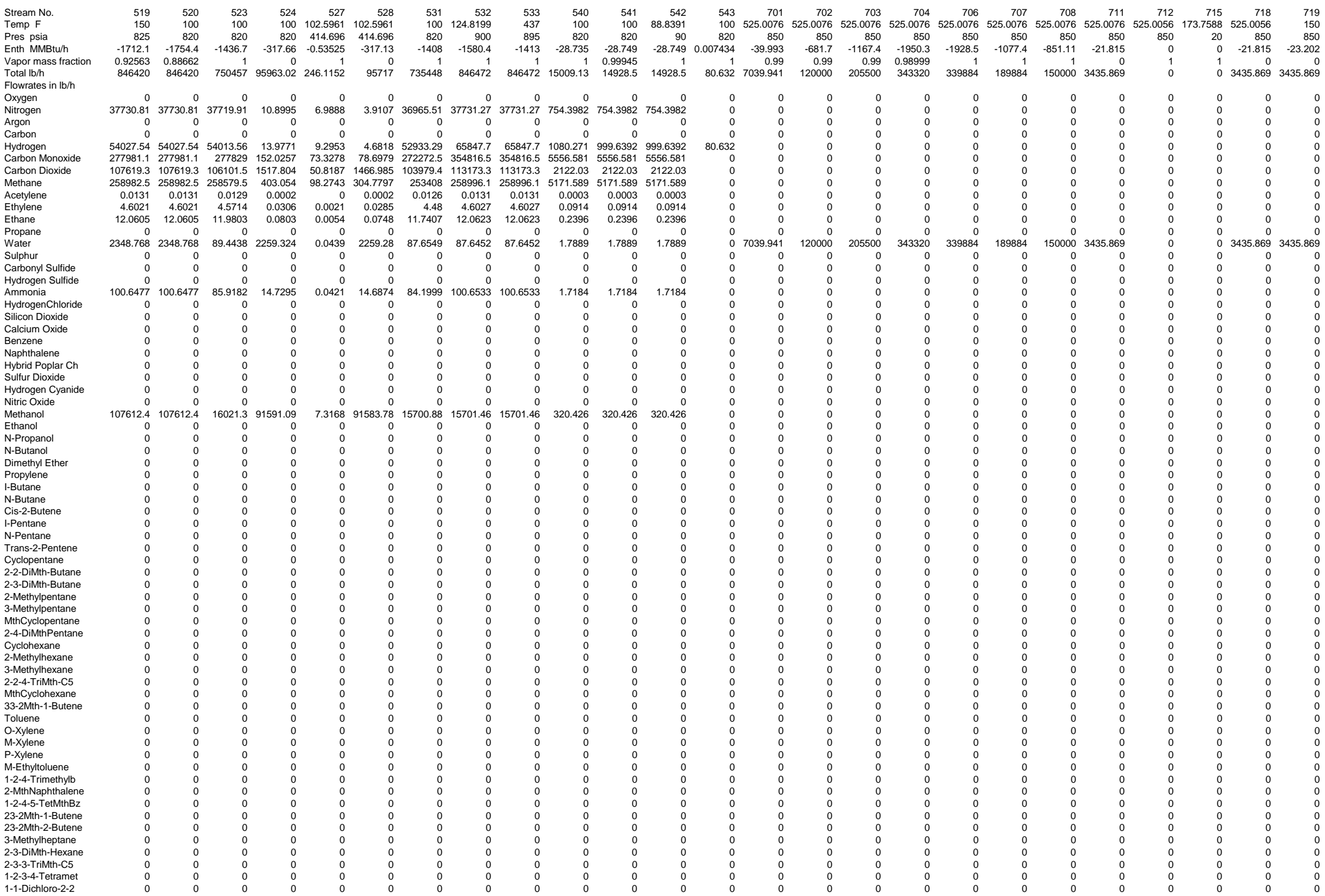




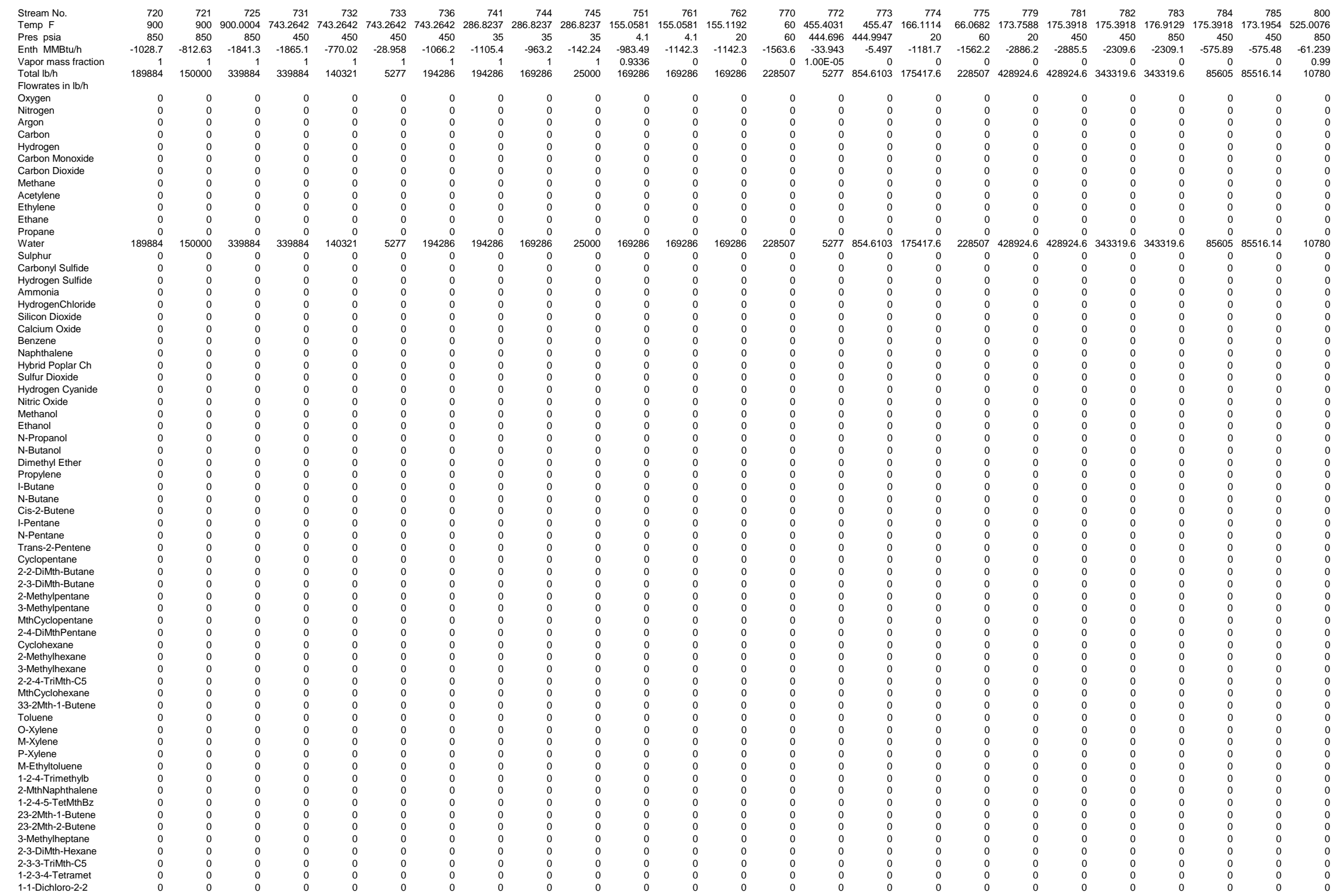




\begin{tabular}{|c|c|c|c|c|c|c|c|c|c|c|c|c|c|c|c|c|c|c|c|c|c|c|c|c|c|}
\hline ream No. & & & 1602 & 1701 & 1702 & 1703 & 1741 & 1742 & 1743 & 1744 & 1745 & 1746 & 1747 & 1748 & 1749 & 1750 & 1830 & 1831 & 1834 & 1835 & 1840 & 1841 & 1845 & 1846 & $\begin{array}{r}1850 \\
90\end{array}$ \\
\hline emp $F$ & 117.8229 & 42.0944 & 43.9445 & 118.1274 & 118.1274 & 118.1274 & 252.8497 & 268.9654 & & 194.8721 & 194.8719 & 207.8941 & 203.9674 & 229.1196 & 210.2323 & 170.2928 & $\begin{array}{r}90 \\
90\end{array}$ & 110 & 90 & 110 & 90 & 110 & 90 & 110 & $\begin{array}{l}90 \\
65\end{array}$ \\
\hline $\begin{array}{l}\text { Pres psia } \\
\text { Enth MMBtu/h }\end{array}$ & $\begin{array}{r}110 \\
-7.8046\end{array}$ & $\begin{array}{r}100 \\
-85056\end{array}$ & $\begin{array}{r}90 \\
-41.189\end{array}$ & $\begin{array}{r}15 \\
-7251\end{array}$ & $\begin{array}{r}15 \\
-72521\end{array}$ & $\begin{array}{r}15 \\
-7196\end{array}$ & $\begin{array}{r}28 \\
0\end{array}$ & 54 & $\begin{array}{l}0 \\
0\end{array}$ & $\begin{array}{r}109.5 \\
-16257\end{array}$ & $\begin{array}{r}109.5 \\
-16257\end{array}$ & $\begin{array}{r}220 \\
-37.833\end{array}$ & $\begin{array}{l}109.5 \\
-5409\end{array}$ & $\begin{array}{r}465 \\
-17565\end{array}$ & $\begin{array}{r}109.5 \\
-71946\end{array}$ & $\begin{array}{r}109.5 \\
-120.81\end{array}$ & $\begin{array}{r}60 \\
-29332 \\
\end{array}$ & $\begin{array}{r}60 \\
-29237\end{array}$ & 60 & $\begin{array}{r}60 \\
-5654 \\
-\end{array}$ & $\begin{array}{r}65 \\
-94644\end{array}$ & $\begin{array}{r}65 \\
-94366\end{array}$ & $\begin{array}{r}65 \\
-3132\end{array}$ & $\begin{array}{r}65 \\
-31041\end{array}$ & $\begin{array}{r}65 \\
-28804\end{array}$ \\
\hline $\begin{array}{l}\text { Enth MMBtuluh } \\
\text { Vapor mass fraction }\end{array}$ & & $\begin{array}{l}-8.9056 \\
0.99473\end{array}$ & $\begin{array}{l}-411.189 \\
0.99856\end{array}$ & & & & & $\begin{array}{l}0 \\
0\end{array}$ & & & & & $\begin{array}{r}-54.09 \\
6.03 E-06\end{array}$ & & $\begin{array}{r}-71.946 \\
1.74 \mathrm{E}-05\end{array}$ & 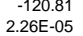 & & & & & & & & & \\
\hline Total lb/h & & 2779.692 & 21273.2 & 10698793 & 106988 & 10591805 & 0 & 0 & & & & & $\begin{array}{l}\text { 8077.098 } \\
8077.098\end{array}$ & 2677.334 & $\begin{array}{l}1.14-0754.43 \\
0.75\end{array}$ & $\begin{array}{l}2.20-05 \\
17958.87\end{array}$ & 4304043 & 4304043 & & 83234.32 & 138920 & 138920 & & 456969.2 & 4227987 \\
\hline $\begin{array}{l}\text { Flowrates in } \mathrm{lb} / \mathrm{h} \\
\text { Oxycen }\end{array}$ & & & & & & & & & & & & & & & & & & & & & & & & & \\
\hline $\begin{array}{l}\text { Nxygen } \\
\text { Nitrogen }\end{array}$ & 0 & 3.8458 & 765.2329 & 0.3386 & 0.0034 & 0.3353 & 0 & 0 & 0 & & 0 & $\begin{array}{l}0 \\
0\end{array}$ & $\begin{array}{l}0 \\
0\end{array}$ & $\begin{array}{l}0 \\
0\end{array}$ & $\begin{array}{r}0 \\
0.0001\end{array}$ & $\begin{array}{r}0 \\
0.0001\end{array}$ & $\begin{array}{l}0 \\
0\end{array}$ & $\begin{array}{l}0 \\
0\end{array}$ & $\begin{array}{l}0 \\
0\end{array}$ & $\begin{array}{l}0 \\
0\end{array}$ & & $\begin{array}{l}0 \\
0\end{array}$ & $\begin{array}{l}0 \\
0\end{array}$ & $\begin{array}{l}0 \\
0\end{array}$ & \\
\hline Argon & 0 & & & & & 0 & 0 & 0 & 0 & 0 & 0 & 0 & 0 & 0 & 0 & 0 & 0 & 0 & 0 & 0 & 0 & 0 & 0 & 0 & \\
\hline Carbon & & & & & & & 0 & 0 & 0 & & o & & 0 & 0 & 0 & & 0 & 0 & 0 & 0 & 0 & 0 & 0 & 0 & \\
\hline Hydrogen & & 33.4608 & 1042.396 & 5.1849 & 0.0518 & 5.133 & 0 & 0 & 0 & 0.0006 & 0.0006 & 0.0035 & 0.0041 & 0.005 & 0.0091 & 0.0108 & 0 & 0 & 0 & 0 & 0 & 0 & 0 & 0 & \\
\hline Carbon Monoxide & & 0 & 5629.909 & 81.6545 & 0.8165 & 80.8379 & 0 & & 0 & 0.0039 & 0.0039 & 0.0229 & 0.0268 & 0.0321 & 0.0588 & 0.0702 & 0 & 0 & 0 & 0 & 0 & 0 & 0 & 0 & 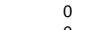 \\
\hline $\begin{array}{l}\text { Carbon Dioxide } \\
\text { Methane }\end{array}$ & & $\begin{array}{l}1863.509 \\
370.4719\end{array}$ & $\begin{array}{l}4036.357 \\
5640.335\end{array}$ & $\begin{array}{l}780.5779 \\
14.2566\end{array}$ & $\begin{array}{l}7.8058 \\
01426\end{array}$ & $\begin{array}{l}772.7721 \\
14114 \\
\end{array}$ & $\begin{array}{l}0 \\
0 \\
0\end{array}$ & $\begin{array}{l}0 \\
0\end{array}$ & $\begin{array}{l}0 \\
0\end{array}$ & $\begin{array}{r}0.681 \\
0.0035\end{array}$ & $\begin{array}{r}0.681 \\
0.0035\end{array}$ & $\begin{array}{l}2.9869 \\
0.0193\end{array}$ & $\begin{array}{l}3.6679 \\
0.0228\end{array}$ & $\begin{array}{l}2.6135 \\
0.038\end{array}$ & $\begin{array}{l}6.2814 \\
0.046\end{array}$ & $\begin{array}{l}25.2043 \\
\end{array}$ & 0 & 0 & 0 & $0_{0}^{0}$ & 0 & 0 & 0 & 0 & $\begin{array}{l}0 \\
0\end{array}$ \\
\hline $\begin{array}{l}\text { Methane } \\
\text { Acetylene }\end{array}$ & & $\begin{array}{rl}370.4719 & 0 \\
0\end{array}$ & $\begin{array}{r}5640.335 \\
0.0003\end{array}$ & $\begin{array}{r}14.2566 \\
12.507\end{array}$ & $\begin{array}{l}0.1426 \\
0.1251\end{array}$ & $\begin{array}{l}14.114 \\
12.382\end{array}$ & $\begin{array}{l}0 \\
0\end{array}$ & $\begin{array}{l}0 \\
0\end{array}$ & $\begin{array}{l}0 \\
0\end{array}$ & $\begin{array}{l}0.0035 \\
0.0013\end{array}$ & $\begin{array}{l}0.0035 \\
0.0013\end{array}$ & $\begin{array}{l}0.0193 \\
0.0064\end{array}$ & $\begin{array}{l}0.0228 \\
0.0077\end{array}$ & 0.0238 & $\begin{array}{l}0.0466 \\
0.0147\end{array}$ & 0.0628 & $\begin{array}{l}0 \\
0\end{array}$ & $\begin{array}{l}0 \\
0\end{array}$ & $\begin{array}{l}0 \\
0\end{array}$ & $\begin{array}{l}0 \\
0\end{array}$ & 0 & $\begin{array}{l}0 \\
0 \\
0\end{array}$ & 0 & $\begin{array}{l}0 \\
0 \\
0\end{array}$ & $\begin{array}{l}0 \\
0 \\
0\end{array}$ \\
\hline $\begin{array}{l}\text { Acctylene } \\
\text { Ethylene }\end{array}$ & & $\begin{array}{r}0 \\
11.8184\end{array}$ & $\begin{array}{l}0.0003 \\
11.912 \\
\end{array}$ & $\begin{array}{r}12.507 \\
66.2334\end{array}$ & $\begin{array}{l}0.1251 \\
0.6623\end{array}$ & $\begin{array}{r}12.2832 \\
65.5711\end{array}$ & $\begin{array}{l}0 \\
0\end{array}$ & 0 & 0 & 0.003 & 0.003 & $\begin{array}{l}0.0064 \\
0.0165\end{array}$ & 0.0195 & 0.0202 & $\begin{array}{l}0.0147 \\
0.0397\end{array}$ & 0.0521 & & 0 & 0 & 0 & 0 & & & & \\
\hline $\begin{array}{l}\text { Enryyene } \\
\text { Ethane }\end{array}$ & & $\begin{array}{l}11.1 .1864 \\
31.6736\end{array}$ & $\begin{array}{l}11.912 \\
31.9187\end{array}$ & $\begin{array}{r}0.2334 \\
0.8156\end{array}$ & $\begin{array}{l}0.0023 \\
0.0082\end{array}$ & $\begin{array}{l}0.5111 \\
0.8075\end{array}$ & 0 & 0 & 0 & & 0.003 & 0.0002 & 0.0002 & 0.0003 & 0.0005 & 0.0006 & 0 & 0 & 0 & 0 & 0 & 0 & 0 & 0 & \\
\hline $\begin{array}{l}\text { Propane } \\
\text { Prone }\end{array}$ & 1437.236 & 297.2563 & 923.1663 & & & & 0 & 0 & 0 & & & & 0.000 & & $\begin{array}{r}0.0000 \\
0\end{array}$ & & & 0 & 0 & 0 & & 0 & 0 & 0 & \\
\hline Water & & 16.9057 & 18.7381 & 10685664 & 106856.6 & 10578807 & 0 & 0 & 0 & 2423.249 & 2423.249 & 5649.425 & 8072.674 & 2674.15 & 10746.82 & 17929.4 & 4304043 & 4304043 & 83234.32 & 83234.32 & 138920 & 138920 & 456969.2 & 456969.2 & \\
\hline ulphur & 0 & 0 & 0 & 0 & 0 & 0 & 0 & 0 & 0 & 0 & 0 & 0 & 0 & 0 & & 0 & & 0 & 0 & 0 & 0 & 0 & 0 & 0 & 0 \\
\hline arbonyl Sulfide & & & & 0 & $\begin{array}{r}0 \\
0.0797\end{array}$ & $\begin{array}{r}0 \\
10483\end{array}$ & 0 & 0 & 0 & $\begin{array}{r}0 \\
0\end{array}$ & 0 & $\begin{array}{r}0 \\
0\end{array}$ & 0 & $\begin{array}{r}0 \\
0\end{array}$ & $\begin{aligned} 0 \\
0\end{aligned}$ & & 0 & 0 & 0 & 0 & & 0 & & 0 & 0 \\
\hline $\begin{array}{l}\text { Hydrogene Sulfide } \\
\text { Ammonia }\end{array}$ & $\begin{array}{l}0 \\
0\end{array}$ & $\begin{array}{l}0 \\
0\end{array}$ & $\begin{array}{r}0 \\
1.7605\end{array}$ & $\begin{array}{r}1.968 \\
10223.75\end{array}$ & $\begin{array}{r}0.0197 \\
102.2375\end{array}$ & $\begin{array}{r}1.9483 \\
10121.51\end{array}$ & $\begin{array}{l}0 \\
0\end{array}$ & $\begin{array}{l}0 \\
0\end{array}$ & $\begin{array}{l}0 \\
0\end{array}$ & $\begin{array}{l}0.0034 \\
0.1179\end{array}$ & $\begin{array}{l}0.0034 \\
0.1179\end{array}$ & $\begin{array}{l}0.0159 \\
0.5142\end{array}$ & $\begin{array}{l}0.0193 \\
0.6321\end{array}$ & $\begin{array}{l}.0 .0155 \\
0.4425\end{array}$ & $\begin{array}{l}0.00488 \\
1.0746\end{array}$ & $\begin{array}{r}0.079 \\
3.9066\end{array}$ & $\begin{array}{l}0 \\
0\end{array}$ & $\begin{array}{l}0 \\
0\end{array}$ & $\begin{array}{l}0 \\
0\end{array}$ & $\begin{array}{l}0 \\
0\end{array}$ & $\begin{array}{l}0 \\
0 \\
0\end{array}$ & $\begin{array}{l}0 \\
0\end{array}$ & $\begin{array}{l}0 \\
0 \\
0\end{array}$ & $\begin{array}{l}0 \\
0\end{array}$ & $\begin{array}{l}0 \\
0\end{array}$ \\
\hline HydrogenChloride & 0 & & & 1852.949 & 18.5295 & 1834.419 & 0 & 0 & 0 & 0 & 0 & 0 & & 0 & 0 & & 0 & 0 & 0 & 0 & 0 & 0 & 0 & 0 & 0 \\
\hline Silicon Dioxide & & & & 0 & 0 & 0 & 0 & 0 & 0 & 0 & 0 & 0 & 0 & 0 & 0 & 0 & 0 & 0 & 0 & 0 & 0 & 0 & 0 & 0 & 0 \\
\hline $\begin{array}{l}\text { Calcium Oxide } \\
\text { Benzene }\end{array}$ & $\begin{array}{r}0 \\
0.0001\end{array}$ & $\begin{array}{r}0 \\
01397\end{array}$ & $\begin{array}{r}0 \\
01397\end{array}$ & $\begin{array}{r}0 \\
253799\end{array}$ & $\begin{array}{r}0 \\
0.2538\end{array}$ & $\begin{aligned} 0 \\
251261\end{aligned}$ & 0 & $\begin{array}{l}0 \\
0\end{array}$ & $\begin{array}{l}0 \\
0\end{array}$ & $\begin{array}{r}0 \\
0.0022\end{array}$ & $\begin{array}{r}0 \\
0.0022\end{array}$ & $\begin{array}{r}0 \\
0.12\end{array}$ & $\begin{array}{r}0 \\
0.143\end{array}$ & $\begin{array}{r}0 \\
0.0141\end{array}$ & $\begin{array}{r}0 \\
0.0284\end{array}$ & $\begin{array}{r}0 \\
0.0358\end{array}$ & $\begin{array}{l}0 \\
0\end{array}$ & 0 & 0 & 0 & 0 & & & & 0 \\
\hline & & $\begin{array}{l}0.1397 \\
0.1495\end{array}$ & $\begin{array}{l}0.11397 \\
0.1495\end{array}$ & $\begin{array}{r}25.3799 \\
63.226\end{array}$ & $\begin{array}{l}0.2538 \\
0.6323\end{array}$ & $\begin{array}{l}25.1261 \\
62.5937\end{array}$ & $\begin{array}{l}0 \\
0\end{array}$ & $\begin{array}{l}0 \\
0\end{array}$ & $\begin{array}{l}0 \\
0\end{array}$ & $\begin{array}{l}0.0022 \\
0.0014\end{array}$ & $\begin{array}{l}0.0022 \\
0.0014\end{array}$ & $\begin{array}{l}0.012 \\
0.0082\end{array}$ & $\begin{array}{l}0.00143 \\
0.0096\end{array}$ & $\begin{array}{l}0.0141 \\
0.0102\end{array}$ & $\begin{array}{l}0.0284 \\
0.0198\end{array}$ & $\begin{array}{r}0.0358 \\
0.022\end{array}$ & $\begin{array}{l}0 \\
0\end{array}$ & $\begin{array}{l}0 \\
0\end{array}$ & $\begin{array}{l}0 \\
0\end{array}$ & $\begin{array}{l}0 \\
0\end{array}$ & $\begin{array}{l}0 \\
0 \\
0\end{array}$ & $\begin{array}{l}0 \\
0\end{array}$ & & $\begin{array}{l}0 \\
0\end{array}$ & $\begin{array}{l}0 \\
0\end{array}$ \\
\hline Hybrid Poplar Ch & 0 & & & & & & 0 & 0 & 0 & $\begin{array}{r}0.0014 \\
0\end{array}$ & $\begin{array}{r}0.0014 \\
0\end{array}$ & $\begin{array}{r}0.0082 \\
0\end{array}$ & $\begin{array}{r}0.0096 \\
0\end{array}$ & $\begin{array}{l}0.0102 \\
0\end{array}$ & & $\begin{array}{r}0.022 \\
0\end{array}$ & 0 & 0 & 0 & 0 & 0 & 0 & & 0 & \\
\hline Sulfur Dioxide & 0 & 0 & & 0 & 0 & 0 & 0 & 0 & 0 & 0 & 0 & 0 & 0 & 0 & 0 & 0 & 0 & 0 & 0 & 0 & 0 & 0 & & & \\
\hline Hydrogen Cyanide & 0 & 0 & 0 & 0 & 0 & 0 & 0 & 0 & 0 & 0 & 0 & 0 & 0 & 0 & 0 & 0 & 0 & 0 & 0 & 0 & 0 & 0 & 0 & 0 & \\
\hline Nitric Oxide & & & 0 & 0 & 0 & 0 & 0 & 0 & 0 & 0 & 0 & 0 & 0 & 0 & 0 & 0 & 0 & 0 & 0 & 0 & 0 & 0 & & 0 & \\
\hline lethanol & 0 & & $\begin{array}{l}327.7415 \\
0\end{array}$ & $\begin{array}{l}0 \\
0\end{array}$ & $\begin{array}{l}0 \\
0 \\
0\end{array}$ & 0 & 0 & 0 & 0 & 0 & 0 & 0 & 0 & 0 & 0 & 0 & 0 & 0 & 0 & 0 & 0 & & & & \\
\hline $\begin{array}{l}\text { hanol } \\
\text { Propanol }\end{array}$ & & & & 0 & $\begin{array}{l}0 \\
0 \\
0\end{array}$ & $\begin{array}{l}0 \\
0\end{array}$ & $\begin{array}{l}0 \\
0\end{array}$ & $\begin{array}{l}0 \\
0\end{array}$ & $\begin{array}{l}0 \\
0\end{array}$ & $\begin{array}{l}0 \\
0 \\
0\end{array}$ & $\begin{array}{l}0 \\
0 \\
0\end{array}$ & $\begin{array}{l}0 \\
0 \\
0\end{array}$ & $\begin{array}{l}0 \\
0\end{array}$ & 0 & 0 & $\begin{array}{l}0 \\
0 \\
0\end{array}$ & $\begin{array}{l}0 \\
0\end{array}$ & $\begin{array}{l}0 \\
0\end{array}$ & $\begin{array}{l}0 \\
0\end{array}$ & $\begin{array}{l}0 \\
0\end{array}$ & 0 & 0 & & $\begin{array}{l}0 \\
0 \\
0\end{array}$ & \\
\hline N-Butanol & & & & 0 & 0 & 0 & 0 & 0 & 0 & 0 & 0 & 0 & $\begin{array}{l}0 \\
0\end{array}$ & 0 & 0 & $\begin{array}{l}0 \\
0 \\
0\end{array}$ & 0 & 0 & $\begin{array}{l}0 \\
0\end{array}$ & $\begin{array}{l}0 \\
0\end{array}$ & $\begin{array}{l}0 \\
0 \\
0\end{array}$ & $\begin{array}{l}0 \\
0 \\
0\end{array}$ & & $\begin{array}{l}0 \\
0\end{array}$ & \\
\hline Dimethyl Ether & 0 & & 0 & 0 & 0 & 0 & 0 & 0 & 0 & 0 & 0 & 0 & 0 & 0 & 0 & 0 & 0 & 0 & 0 & 0 & 0 & 0 & & 0 & \\
\hline pylene & 10.4122 & 6.1225 & 10.657 & 0 & 0 & 0 & 0 & 0 & 0 & 0 & 0 & 0 & 0 & 0 & 0 & 0 & 0 & 0 & 0 & 0 & 0 & 0 & & 0 & 0 \\
\hline uttane & 3411.84 & 48.0827 & 1533.925 & 0 & 0 & 0 & 0 & 0 & 0 & 0 & 0 & 0 & 0 & 0 & 0 & 0 & 0 & 0 & 0 & 0 & 0 & 0 & & & 0 \\
\hline & 1013.892 & 20.4313 & 461.9769 & 0 & 0 & 0 & 0 & 0 & 0 & 0 & 0 & 0 & 0 & 0 & 0 & 0 & & 0 & 0 & 0 & 0 & 0 & 0 & 0 & \\
\hline Cis-2-Butene & 564.7487 & 13.6119 & 259.5576 & 0 & 0 & 0 & 0 & 0 & 0 & 0 & 0 & 0 & 0 & 0 & 0 & 0 & 0 & 0 & 0 & 0 & 0 & 0 & & 0 & 0 \\
\hline N-Pent & $\begin{array}{r}993.9574 \\
65.4574\end{array}$ & $\begin{array}{r}34.1474 \\
57402\end{array}$ & $\begin{array}{r}467.0116 \\
342486\end{array}$ & $\begin{array}{l}0 \\
0\end{array}$ & 0 & 0 & 0 & 0 & 0 & $\begin{array}{l}0 \\
0 \\
0\end{array}$ & 0 & 0 & 0 & 0 & $\begin{array}{l}0 \\
0\end{array}$ & 0 & $\begin{array}{l}0 \\
0\end{array}$ & 0 & 0 & $\begin{array}{l}0 \\
0 \\
0\end{array}$ & ${ }_{0}^{0}$ & 0 & 0 & 0 & $\begin{array}{l}0 \\
0\end{array}$ \\
\hline ntene & $\begin{array}{r}03.4074 \\
116.4683\end{array}$ & $\begin{array}{l}5.1 .42 \\
6.6397\end{array}$ & $\begin{array}{l}34.2485 \\
57.3611\end{array}$ & 0 & 0 & 0 & & 0 & 0 & 0 & 0 & 0 & 0 & 0 & 0 & 然 & 0 & 0 & 0 & 0 & 0 & 0 & & & \\
\hline Cyclo & 0.1416 & 0.396 & 0.4577 & 0 & 0 & 0 & 0 & 0 & 0 & 0 & 0 & 0 & 0 & 0 & 0 & 0 & 0 & 0 & 0 & $\begin{array}{l}0 \\
0 \\
0\end{array}$ & $\begin{array}{l}0 \\
0\end{array}$ & $\begin{array}{l}0 \\
0\end{array}$ & & & \\
\hline tane & 3 & 3.4898 & 3.6 & 0 & 0 & 0 & 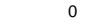 & 0 & 0 & 0 & 0 & 0 & 0 & 0 & 0 & 0 & 0 & 0 & 0 & 0 & 0 & 0 & & & \\
\hline & 0.0114 & 1.828 & 1.833 & 0 & 0 & 0 & & 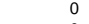 & 0 & 0 & 0 & 0 & 0 & 0 & 0 & 0 & 0 & 0 & 0 & 0 & 0 & 0 & & 0 & 0 \\
\hline & 0.0055 & 2.5315 & 2.5339 & 0 & 0 & 0 & 0 & 0 & 0 & 0 & 0 & 0 & 0 & 0 & 0 & 0 & 0 & 0 & 0 & 0 & 0 & 0 & & 0 & 0 \\
\hline & 0.0016 & & & 0 & 0 & 0 & 0 & 0 & 0 & 0 & 0 & 0 & 0 & 0 & 0 & 0 & 0 & 0 & 0 & 0 & 0 & 0 & & 0 & 0 \\
\hline MthCyclopen & 0 & 0.2 & 0.2 & $\begin{array}{l}0 \\
0\end{array}$ & $\begin{array}{l}0 \\
0\end{array}$ & $\begin{array}{l}0 \\
0\end{array}$ & $\begin{array}{l}0 \\
0 \\
0\end{array}$ & 0 & 0 & 0 & 0 & 0 & 0 & 0 & 0 & 0 & 0 & 0 & 0 & 0 & 0 & 0 & & 0 & 0 \\
\hline & & & & $\begin{array}{l}0 \\
0\end{array}$ & 0 & 0 & & 0 & 0 & $\begin{array}{l}0 \\
0 \\
0\end{array}$ & $0_{0}^{0}$ & 0 & $\begin{array}{l}0 \\
0\end{array}$ & 0 & 0 & 0 & $\begin{array}{l}0 \\
0\end{array}$ & 0 & 0 & $\begin{array}{l}0 \\
0 \\
0\end{array}$ & 0 & 0 & & 0 & $\begin{array}{l}0 \\
0 \\
0\end{array}$ \\
\hline & 0 & 0.0 & & 0 & 0 & 0 & & 0 & 0 & 0 & 0 & 0 & 0 & $\begin{array}{l}0 \\
0\end{array}$ & $\begin{array}{l}0 \\
0\end{array}$ & $\begin{array}{l}0 \\
0 \\
0\end{array}$ & $\begin{array}{l}0 \\
0\end{array}$ & 0 & $\begin{array}{l}0 \\
0\end{array}$ & $\begin{array}{l}0 \\
0 \\
0\end{array}$ & $\begin{array}{l}0 \\
0 \\
0\end{array}$ & 0 & & & $\begin{array}{l}0 \\
0 \\
0\end{array}$ \\
\hline 3-Metn & 0 & 0.0591 & 0.0591 & 0 & 0 & 0 & 0 & 0 & 0 & 0 & 0 & 0 & 0 & 0 & 0 & 0 & 0 & 0 & 0 & 0 & o & & & 0 & 0 \\
\hline & 0 & & & 0 & 0 & & & & & & 0 & & & 0 & 0 & 0 & & 0 & 0 & 0 & 0 & 0 & & & 0 \\
\hline WthCycl & & 0.0322 & 0.0 & 0 & 0 & 0 & & 0 & 0 & 0 & 0 & 0 & 0 & 0 & 0 & 0 & 0 & 0 & 0 & 0 & 0 & 0 & 0 & 0 & c \\
\hline & 6.4445 & 2.8 & & 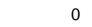 & 0 & 0 & & 0 & 0 & 0 & 0 & 0 & 0 & 0 & 0 & 0 & 0 & 0 & 0 & 0 & 0 & 0 & & & 0 \\
\hline Tol & 0 & 0.0 & 0.0 & 0 & 0 & 0 & & 0 & 0 & 0 & 0 & 0 & 0 & 0 & 0 & 0 & 0 & 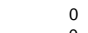 & 0 & 0 & 0 & 0 & 0 & 0 & 0 \\
\hline & 0 & 0.0 & & 0 & 0 & 0 & & 0 & 0 & 0 & 0 & 0 & 0 & 0 & 0 & 0 & 0 & 0 & 0 & 0 & 0 & 0 & & 0 & \\
\hline & 0 & & & 0 & 0 & 0 & & 0 & 0 & 0 & 0 & 0 & 0 & 0 & 0 & 0 & 0 & 0 & 0 & 0 & 0 & 0 & 0 & 0 & 0 \\
\hline 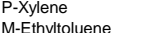 & $\begin{array}{l}0 \\
0 \\
0\end{array}$ & & $\begin{array}{l}0.0 \\
0.0\end{array}$ & $\begin{array}{l}0 \\
0\end{array}$ & $\begin{array}{l}0 \\
0\end{array}$ & $\begin{array}{l}0 \\
0\end{array}$ & & $\begin{array}{l}0 \\
0 \\
0\end{array}$ & $\begin{array}{l}0 \\
0 \\
0\end{array}$ & $\begin{array}{l}0 \\
0 \\
0\end{array}$ & $\begin{array}{l}0 \\
0 \\
0\end{array}$ & $\begin{array}{l}0 \\
0\end{array}$ & $\begin{array}{l}0 \\
0\end{array}$ & $\begin{array}{l}0 \\
0\end{array}$ & $\begin{array}{l}0 \\
0\end{array}$ & $\begin{array}{l}0 \\
0 \\
0\end{array}$ & $\begin{array}{l}0 \\
0\end{array}$ & $\begin{array}{l}0 \\
0\end{array}$ & $\begin{array}{l}0 \\
0\end{array}$ & $\begin{array}{l}0 \\
0 \\
0\end{array}$ & 0 & 0 & & & 0 \\
\hline & 0 & 0.0004 & 0.0 & 0 & 0 & 0 & 0 & 0 & 0 & 0 & 0 & 0 & 0 & 0 & 0 & 0 & 0 & 0 & 0 & 0 & 0 & 0 & 0 & 0 & 0 \\
\hline e & 0 & 0.0071 & 0.0071 & 0 & 0 & 0 & 0 & 0 & 0 & 0 & 0 & 0 & 0 & 0 & 0 & 0 & 0 & 0 & 0 & 0 & 0 & 0 & 0 & 0 & 0 \\
\hline & 0 & 00 & 10 & 0 & 0 & 0 & & 0 & 0 & ${ }_{0}^{0}$ & $0_{0}^{0}$ & 0 & 0 & 0 & 0 & 0 & 0 & 0 & 0 & 0 & 0 & 0 & 0 & 0 & \\
\hline & $\begin{array}{l}0.0213 \\
0\end{array}$ & $\begin{array}{l}0.9968 \\
0.2532\end{array}$ & $\begin{array}{l}1.0 \\
0.2\end{array}$ & $\begin{array}{l}0 \\
0\end{array}$ & $\begin{array}{l}0 \\
0\end{array}$ & $\begin{array}{l}0 \\
0\end{array}$ & 0 & 0 & 0 & $\begin{array}{l}0 \\
0\end{array}$ & 0 & $\begin{array}{l}0 \\
0\end{array}$ & 0 & 0 & 0 & 然 & 0 & 0 & 0 & 0 & $\begin{array}{l}0 \\
0\end{array}$ & 0 & $\begin{array}{l}0 \\
0\end{array}$ & $\begin{array}{l}0 \\
0\end{array}$ & \\
\hline & 0 & & & 0 & 0 & 0 & 0 & 0 & 0 & 0 & 0 & 0 & 0 & 0 & 0 & & & 0 & 0 & 0 & & & & & \\
\hline & 0 & 0.0063 & 0.0 & 0 & 0 & 0 & 0 & 0 & 0 & 0 & 0 & 0 & 0 & 0 & 0 & 0 & 0 & 0 & 0 & 0 & 0 & 0 & 0 & 0 & \\
\hline & 0 & 0.008 & 0. & 0 & 0 & 0 & 0 & 0 & 0 & 0 & 0 & 0 & 0 & 0 & 0 & 0 & 0 & 0 & 0 & 0 & 0 & 0 & & 0 & \\
\hline 1-2-3-3-4tetramet & 0 & 1.2974 & 1.2974 & $\begin{array}{l}0 \\
0\end{array}$ & $\begin{array}{l}0 \\
0\end{array}$ & $\begin{array}{l}0 \\
0\end{array}$ & $\begin{array}{l}0 \\
0\end{array}$ & $\begin{array}{l}0 \\
0\end{array}$ & $\begin{array}{l}0 \\
0\end{array}$ & 0 & $\begin{array}{l}0 \\
0\end{array}$ & $\begin{array}{l}0 \\
0 \\
0\end{array}$ & $\begin{array}{l}0 \\
0\end{array}$ & $\begin{array}{l}0 \\
0\end{array}$ & $\begin{array}{l}0 \\
0\end{array}$ & 0 & 0 & 0 & 0 & 0 & 0 & 0 & 0 & 0 & \\
\hline
\end{tabular}




\begin{tabular}{|c|c|c|c|c|c|c|c|c|c|c|c|c|c|c|c|c|c|c|c|c|c|c|c|c|}
\hline Stream No. & & & 1910 & 1931 & 1932 & 1935 & 1936 & & & 1941 & & & & & & & 1950 & & & & & & & \\
\hline Temp F & 100 & 456.586 & 456.586 & 176 & 525.0076 & 176 & & 525.0076 & 743.0002 & 456.5881 & 455.4713 & 456.586 & 900 & 525.0096 & 525.0096 & 900 & 176 & 525.0076 & 456 & 456.586 & 456.586 & 743.0002 & 456.586 & 456.586 \\
\hline Pres psia & & & & 850 & & 850 & 850 & 850 & & & 445 & 450 & 850 & 850 & & 850 & 850 & 850 & 450 & 450 & 450 & 450 & 450 & 450 \\
\hline Enth MMBtu/h & -28762 & -414.33 & -447.89 & -47.356 & -39.993 & -807.2 & -779.34 & -681.7 & -770.04 & -4.8423 & -5.4961 & -3.2036 & -1028.7 & -1077.4 & -851.11 & -812.63 & -1382.3 & -1167.4 & -772.5 & -681.52 & -484.4 & -28.964 & -33.942 & -62.93 \\
\hline $\begin{array}{l}\text { Vapor mass fraction } \\
\text { Total lb/h }\end{array}$ & $\begin{array}{r}0 \\
4227987\end{array}$ & $\begin{array}{r}1 \\
73121\end{array}$ & $\begin{array}{r}0.39969 \\
73121.01\end{array}$ & $\begin{array}{r}0 \\
7039941\end{array}$ & $\begin{array}{r}0.99 \\
7039.941\end{array}$ & $\begin{array}{r}0 \\
120000\end{array}$ & $\begin{array}{r}0 \\
120000\end{array}$ & $\begin{array}{r}0.99 \\
120000\end{array}$ & $\begin{array}{r}1 \\
140321\end{array}$ & $\begin{array}{r}1 \\
854.5667\end{array}$ & $\begin{array}{r}0.001 \\
854.5667\end{array}$ & 0.39969 & $\begin{array}{r}1 \\
189884\end{array}$ & $\begin{array}{r}1 \\
189884\end{array}$ & $\begin{array}{r}1 \\
150000\end{array}$ & $\begin{array}{r}1 \\
150000\end{array}$ & $\begin{array}{r}0 \\
205500\end{array}$ & $\begin{array}{r}0.99 \\
205500\end{array}$ & $\begin{array}{r}0 \\
1201098\end{array}$ & $\begin{array}{r}0.98976 \\
1201098\end{array}$ & $\begin{array}{r}1 \\
8548684\end{array}$ & $\begin{array}{r}1 \\
5277.925\end{array}$ & $\begin{array}{l}1.00 \mathrm{E}-06 \\
5277.925\end{array}$ & $\begin{array}{r}1 \\
11106\end{array}$ \\
\hline $\begin{array}{l}\text { Tlowrates in } \mathrm{lb} / \mathrm{h} \\
\text { Flown }\end{array}$ & & & & & & & & & & & & & & & & & & & & & & & & \\
\hline Oxygen & 0 & 0 & 0 & 0 & 0 & 0 & 0 & 0 & 0 & 0 & 0 & 0 & 0 & 0 & 0 & 0 & 0 & 0 & 0 & 0 & 0 & 0 & 0 & 0 \\
\hline Nitrogen & 0 & 0 & 0 & & & 0 & & & 0 & 0 & 0 & & 0 & 0 & & & 0 & & & & & 0 & & 0 \\
\hline Argon & 0 & 0 & 0 & 0 & 0 & 0 & 0 & 0 & 0 & 0 & 0 & 0 & 0 & 0 & 0 & 0 & 0 & 0 & 0 & 0 & 0 & 0 & 0 & 0 \\
\hline Carbon & 0 & 0 & 0 & 0 & 0 & 0 & 0 & 0 & 0 & 0 & 0 & 0 & 0 & 0 & 0 & 0 & 0 & 0 & 0 & 0 & 0 & 0 & 0 & 0 \\
\hline Hydrogen & 0 & 0 & 0 & 0 & 0 & 0 & 0 & 0 & 0 & 0 & 0 & 0 & 0 & 0 & 0 & 0 & 0 & 0 & 0 & 0 & 0 & 0 & & 0 \\
\hline $\begin{array}{l}\text { Carbon Monoxide } \\
\text { Carbon Dioxide }\end{array}$ & 0 & $\begin{array}{l}0 \\
0\end{array}$ & 0 & 0 & 0 & 0 & 0 & 0 & 0 & $\begin{array}{l}0 \\
0\end{array}$ & $\begin{array}{l}0 \\
0\end{array}$ & 0 & 0 & 0 & $0_{0}^{0}$ & 0 & 0 & $0_{0}^{0}$ & 0 & 0 & 0 & 0 & 0 & 0 \\
\hline $\begin{array}{l}\text { Carbon Dioxide } \\
\text { Methane }\end{array}$ & $\begin{array}{l}0 \\
0 \\
0\end{array}$ & 0 & $\begin{array}{l}0 \\
0 \\
0\end{array}$ & $\begin{array}{l}0 \\
0 \\
0\end{array}$ & $\begin{array}{l}0 \\
0\end{array}$ & $\begin{array}{l}0 \\
0\end{array}$ & $\begin{array}{l}0 \\
0 \\
0\end{array}$ & $\begin{array}{l}0 \\
0 \\
0\end{array}$ & $\begin{array}{l}0 \\
0\end{array}$ & 0 & 0 & 0 & $\begin{array}{l}0 \\
0 \\
0\end{array}$ & 0 & 0 & 0 & 0 & 0 & 0 & 0 & 0 & 0 & 0 & 0 \\
\hline $\begin{array}{l}\text { Metnane } \\
\text { Acetylene }\end{array}$ & $\begin{array}{l}0 \\
0\end{array}$ & $\begin{array}{l}0 \\
0\end{array}$ & $\begin{array}{l}0 \\
0\end{array}$ & $\begin{array}{l}0 \\
0 \\
0\end{array}$ & $\begin{array}{l}0 \\
0\end{array}$ & $\begin{array}{l}0 \\
0\end{array}$ & $\begin{array}{l}0 \\
0\end{array}$ & $\begin{array}{l}0 \\
0\end{array}$ & 0 & $\begin{array}{l}0 \\
0\end{array}$ & $\begin{array}{l}0 \\
0\end{array}$ & 0 & $\begin{array}{l}0 \\
0\end{array}$ & 0 & 0 & 0 & 0 & $\begin{array}{l}0 \\
0\end{array}$ & 然 & 0 & $\begin{array}{l}0 \\
0\end{array}$ & 0 & 0 & $\begin{array}{l}0 \\
0\end{array}$ \\
\hline $\begin{array}{l}\text { Acelylene } \\
\text { Ethylene }\end{array}$ & 0 & 0 & 0 & 0 & 0 & 0 & 0 & 0 & 0 & 0 & 0 & 0 & 0 & 0 & 0 & 0 & 0 & 0 & 0 & 0 & 0 & 0 & 0 & 0 \\
\hline Ethane & 0 & 0 & 0 & 0 & 0 & 0 & 0 & 0 & 0 & 0 & 0 & 0 & 0 & 0 & 0 & 0 & 0 & 0 & 0 & 0 & 0 & 0 & & 0 \\
\hline $\begin{array}{l}\text { Propane } \\
\text { Whan }\end{array}$ & 0 & 0 & & & 0 & 0 & & & & & & & & & & & & & & 0 & 0 & 0 & 0 & 0 \\
\hline Water & 4227987 & 73121 & 73121.01 & & 7039.941 & 120000 & 120000 & 120000 & 140321 & 854.5667 & 854.5667 & 523 & 189884 & 189884 & 150000 & 150000 & 205500 & 205500 & 120109.8 & 120109.8 & $85486.84 \mathrm{H}$ & & 5277.925 & 11106 \\
\hline Sulphur & 0 & 0 & 0 & 0 & 0 & 0 & 0 & 0 & 0 & 0 & 0 & 0 & 0 & 0 & 0 & 0 & 0 & 0 & 0 & 0 & 0 & 0 & 0 & 0 \\
\hline Carbonyl Sulfide & 0 & 0 & 0 & 0 & 0 & 0 & 0 & 0 & 0 & 0 & 0 & 0 & 0 & 0 & 0 & 0 & 0 & 0 & 0 & 0 & 0 & 0 & 0 & 0 \\
\hline Hydrogen Sulfide & 0 & 0 & 0 & 0 & 0 & 0 & 0 & 0 & 0 & 0 & 0 & 0 & 0 & 0 & 0 & 0 & 0 & 0 & 0 & 0 & 0 & 0 & 0 & 0 \\
\hline Ammonia & 0 & 0 & 0 & 0 & 0 & 0 & 0 & 0 & 0 & 0 & 0 & 0 & 0 & 0 & 0 & 0 & 0 & 0 & 0 & 0 & 0 & 0 & 0 & 0 \\
\hline $\begin{array}{l}\text { HydrogenChloride } \\
\text { Silicon ioxide }\end{array}$ & 0 & 0 & 0 & 0 & 0 & 0 & 0 & 0 & 0 & 0 & 0 & 0 & 0 & 0 & 0 & 0 & 0 & 0 & 0 & 0 & 0 & 0 & 0 & 0 \\
\hline $\begin{array}{l}\text { Silicon Dioxide } \\
\text { Calcium Oxide }\end{array}$ & $\begin{array}{l}0 \\
0\end{array}$ & $\begin{array}{l}0 \\
0\end{array}$ & $\begin{array}{l}0 \\
0 \\
0\end{array}$ & $\begin{array}{l}0 \\
0 \\
0\end{array}$ & 0 & $\begin{array}{l}0 \\
0\end{array}$ & $\begin{array}{l}0 \\
0 \\
0\end{array}$ & $\begin{array}{l}0 \\
0\end{array}$ & $\begin{array}{l}0 \\
0\end{array}$ & $\begin{array}{l}0 \\
0\end{array}$ & $\begin{array}{l}0 \\
0 \\
0\end{array}$ & $\begin{array}{l}0 \\
0 \\
0\end{array}$ & 0 & 0 & 0 & 0 & 0 & 0 & 0 & 0 & 0 & 0 & 0 & 0 \\
\hline $\begin{array}{l}\text { Calcume Oxide } \\
\text { Benzene }\end{array}$ & $\begin{array}{l}0 \\
0\end{array}$ & $\begin{array}{l}0 \\
0\end{array}$ & $\begin{array}{l}0 \\
0\end{array}$ & $\begin{array}{l}0 \\
0\end{array}$ & $\begin{array}{l}0 \\
0\end{array}$ & $\begin{array}{l}0 \\
0\end{array}$ & $\begin{array}{l}0 \\
0\end{array}$ & $\begin{array}{l}0 \\
0\end{array}$ & $\begin{array}{l}0 \\
0\end{array}$ & $\begin{array}{l}0 \\
0\end{array}$ & $\begin{array}{l}0 \\
0\end{array}$ & $\begin{array}{l}0 \\
0\end{array}$ & $\begin{array}{l}0 \\
0\end{array}$ & $\begin{array}{l}0 \\
0\end{array}$ & $\begin{array}{l}0 \\
0\end{array}$ & 0 & $\begin{array}{l}0 \\
0\end{array}$ & $\begin{array}{l}0 \\
0\end{array}$ & $\begin{array}{l}0 \\
0\end{array}$ & $\begin{array}{l}0 \\
0\end{array}$ & $\begin{array}{l}0 \\
0\end{array}$ & $\begin{array}{l}0 \\
0\end{array}$ & $\begin{array}{l}0 \\
0\end{array}$ & $\begin{array}{l}0 \\
0\end{array}$ \\
\hline Naphthalene & 0 & 0 & 0 & 0 & 0 & 0 & 0 & 0 & 0 & 0 & 0 & 0 & 0 & 0 & 0 & 0 & 0 & 0 & 0 & 0 & 0 & 0 & 0 & 0 \\
\hline Hybrid Poplar Ch & 0 & 0 & 0 & 0 & 0 & 0 & 0 & 0 & 0 & 0 & 0 & 0 & 0 & 0 & 0 & 0 & 0 & 0 & 0 & 0 & 0 & 0 & 0 & 0 \\
\hline $\begin{array}{l}\text { Sulfur Dioxide } \\
\text { Hydroneno }\end{array}$ & 0 & 0 & 0 & 0 & 0 & 0 & 0 & 0 & 0 & 0 & 0 & 0 & 0 & 0 & 0 & 0 & 0 & 0 & 0 & 0 & 0 & 0 & 0 & 0 \\
\hline $\begin{array}{l}\text { Hydrogen Cyanide } \\
\text { Niticic Oxide }\end{array}$ & $\begin{array}{l}0 \\
0\end{array}$ & 0 & $\begin{array}{l}0 \\
0\end{array}$ & 0 & 0 & 0 & 0 & 0 & 0 & 0 & 0 & 0 & 0 & 0 & 0 & 0 & 0 & 0 & 0 & 0 & 0 & 0 & 0 & 0 \\
\hline $\begin{array}{l}\text { Nitric Oxide } \\
\text { Methanol }\end{array}$ & $\begin{array}{l}0 \\
0 \\
0\end{array}$ & $\begin{array}{l}0 \\
0\end{array}$ & $\begin{array}{l}0 \\
0 \\
0\end{array}$ & $\begin{array}{l}0 \\
0 \\
0\end{array}$ & $\begin{array}{l}0 \\
0\end{array}$ & $\begin{array}{l}0 \\
0\end{array}$ & $\begin{array}{l}0 \\
0 \\
0\end{array}$ & $\begin{array}{l}0 \\
0\end{array}$ & $\begin{array}{l}0 \\
0\end{array}$ & $\begin{array}{l}0 \\
0 \\
0\end{array}$ & $\begin{array}{l}0 \\
0\end{array}$ & $\begin{array}{l}0 \\
0 \\
0\end{array}$ & $\begin{array}{l}0 \\
0\end{array}$ & $\begin{array}{l}0 \\
0 \\
0\end{array}$ & $\begin{array}{l}0 \\
0\end{array}$ & $\begin{array}{l}0 \\
0\end{array}$ & $\begin{array}{l}0 \\
0\end{array}$ & $\begin{array}{l}0 \\
0 \\
0\end{array}$ & $\begin{array}{l}0 \\
0\end{array}$ & $\begin{array}{l}0 \\
0\end{array}$ & $\begin{array}{l}0 \\
0\end{array}$ & $\begin{array}{l}0 \\
0\end{array}$ & $\begin{array}{l}0 \\
0 \\
0\end{array}$ & 0 \\
\hline $\begin{array}{l}\text { Mentranol } \\
\text { Ethanol }\end{array}$ & 0 & 0 & 0 & 0 & 0 & 0 & 0 & 0 & 0 & 0 & 0 & 0 & 0 & 0 & 0 & 0 & 0 & 0 & 0 & 0 & 0 & 0 & 0 & 0 \\
\hline N-Propanol & 0 & 0 & 0 & 0 & 0 & 0 & 0 & 0 & 0 & 0 & 0 & 0 & 0 & 0 & 0 & 0 & 0 & 0 & 0 & 0 & 0 & 0 & 0 & 0 \\
\hline N-Butanol & 0 & 0 & 0 & 0 & 0 & 0 & 0 & 0 & 0 & 0 & 0 & 0 & 0 & 0 & 0 & 0 & 0 & 0 & 0 & 0 & 0 & 0 & & 0 \\
\hline Dimethyl Ether & 0 & 0 & 0 & 0 & 0 & 0 & & 0 & 0 & 0 & 0 & 0 & 0 & 0 & 0 & 0 & 0 & 0 & 0 & 0 & 0 & 0 & 0 & 0 \\
\hline Propylene & 0 & 0 & 0 & 0 & 0 & 0 & 0 & 0 & 0 & 0 & 0 & 0 & 0 & 0 & 0 & 0 & 0 & 0 & 0 & 0 & 0 & 0 & 0 & 0 \\
\hline I-Butane & 0 & 0 & 0 & 0 & 0 & 0 & 0 & 0 & 0 & 0 & 0 & 0 & 0 & 0 & 0 & 0 & 0 & 0 & 0 & 0 & 0 & 0 & 0 & 0 \\
\hline N-Butane & 0 & 0 & 0 & 0 & 0 & 0 & 0 & 0 & 0 & 0 & 0 & 0 & 0 & 0 & 0 & 0 & 0 & 0 & 0 & 0 & 0 & 0 & 0 & 0 \\
\hline Cis-2-Butene & 0 & 0 & 0 & 0 & 0 & 0 & 0 & 0 & 0 & 0 & 0 & 0 & 0 & 0 & 0 & 0 & 0 & 0 & 0 & 0 & 0 & 0 & 0 & 0 \\
\hline I-Pentane & 0 & 0 & 0 & 0 & 0 & 0 & 0 & 0 & 0 & 0 & 0 & 0 & 0 & 0 & 0 & 0 & 0 & 0 & 0 & 0 & 0 & 0 & 0 & 0 \\
\hline N-Pentane & 0 & 0 & 0 & 0 & 0 & 0 & 0 & 0 & 0 & 0 & 0 & 0 & 0 & 0 & 0 & 0 & 0 & 0 & 0 & 0 & 0 & 0 & 0 & 0 \\
\hline $\begin{array}{l}\text { Trans-2-Pentene } \\
\text { Cyclopentane }\end{array}$ & $\begin{array}{l}0 \\
0 \\
0\end{array}$ & $\begin{array}{l}0 \\
0\end{array}$ & $\begin{array}{l}0 \\
0 \\
0\end{array}$ & $\begin{array}{l}0 \\
0 \\
0\end{array}$ & $\begin{array}{l}0 \\
0\end{array}$ & $\begin{array}{l}0 \\
0\end{array}$ & $\begin{array}{l}0 \\
0 \\
0\end{array}$ & $\begin{array}{l}0 \\
0\end{array}$ & $\begin{array}{l}0 \\
0\end{array}$ & $\begin{array}{l}0 \\
0 \\
0\end{array}$ & $\begin{array}{l}0 \\
0 \\
0\end{array}$ & $\begin{array}{l}0 \\
0 \\
0\end{array}$ & $\begin{array}{l}0 \\
0\end{array}$ & $\begin{array}{l}0 \\
0 \\
0\end{array}$ & $\begin{array}{l}0 \\
0 \\
0\end{array}$ & $\begin{array}{l}0 \\
0\end{array}$ & 0 & 0 & 0 & 0 & $\begin{array}{l}0 \\
0\end{array}$ & 0 & 0 & 0 \\
\hline $\begin{array}{l}\text { Cyclopentane } \\
\text { 2-2-DiMth-Butane }\end{array}$ & 0 & 0 & 0 & 0 & 0 & 0 & 0 & 0 & 0 & 0 & $\begin{array}{l}0 \\
0\end{array}$ & $\begin{array}{l}0 \\
0 \\
0\end{array}$ & $\begin{array}{l}0 \\
0\end{array}$ & $\begin{array}{l}0 \\
0 \\
0\end{array}$ & $\begin{array}{l}0 \\
0 \\
0\end{array}$ & $\begin{array}{l}0 \\
0\end{array}$ & $\begin{array}{l}0 \\
0\end{array}$ & $\begin{array}{l}0 \\
0 \\
0\end{array}$ & $\begin{array}{l}0 \\
0\end{array}$ & $\begin{array}{l}0 \\
0\end{array}$ & $\begin{array}{l}0 \\
0 \\
0\end{array}$ & $\begin{array}{l}0 \\
0 \\
0\end{array}$ & $\begin{array}{l}0 \\
0 \\
0\end{array}$ & $\begin{array}{l}0 \\
0\end{array}$ \\
\hline 2-3-DiMth-Butane & 0 & 0 & 0 & 0 & 0 & 0 & 0 & 0 & 0 & 0 & 0 & 0 & 0 & 0 & 0 & 0 & 0 & 0 & 0 & 0 & 0 & 0 & 0 & 0 \\
\hline 2-Methylpentane & 0 & 0 & 0 & 0 & 0 & 0 & 0 & 0 & 0 & 0 & 0 & 0 & 0 & 0 & 0 & 0 & 0 & 0 & 0 & 0 & 0 & 0 & 0 & 0 \\
\hline & 0 & 0 & 0 & 0 & 0 & 0 & 0 & 0 & 0 & 0 & 0 & 0 & 0 & 0 & 0 & 0 & 0 & 0 & 0 & 0 & 0 & 0 & 0 & 0 \\
\hline MthCyclopentane & $\begin{array}{l}0 \\
0\end{array}$ & 0 & $\begin{array}{l}0 \\
0\end{array}$ & 0 & 0 & 0 & 0 & 0 & 0 & 0 & 0 & 0 & 0 & 0 & 0 & 0 & 0 & 0 & 0 & 0 & 0 & 0 & 0 & 0 \\
\hline $\begin{array}{l}\text { 2-4-2inMthPentane } \\
\text { Cyclohexane }\end{array}$ & $\begin{array}{l}0 \\
0 \\
0\end{array}$ & $\begin{array}{l}0 \\
0\end{array}$ & $\begin{array}{l}0 \\
0\end{array}$ & $\begin{array}{l}0 \\
0 \\
0\end{array}$ & $\begin{array}{l}0 \\
0\end{array}$ & $\begin{array}{l}0 \\
0\end{array}$ & $\begin{array}{l}0 \\
0\end{array}$ & $\begin{array}{l}0 \\
0\end{array}$ & $\begin{array}{l}0 \\
0\end{array}$ & $\begin{array}{l}0 \\
0 \\
0\end{array}$ & $\begin{array}{l}0 \\
0 \\
0\end{array}$ & $\begin{array}{l}0 \\
0 \\
0\end{array}$ & $\begin{array}{l}0 \\
0\end{array}$ & $\begin{array}{l}0 \\
0 \\
0\end{array}$ & $\begin{array}{l}0 \\
0 \\
0\end{array}$ & $\begin{array}{l}0 \\
0\end{array}$ & $\begin{array}{l}0 \\
0\end{array}$ & $\begin{array}{l}0 \\
0 \\
0\end{array}$ & $\begin{array}{l}0 \\
0\end{array}$ & $\begin{array}{l}0 \\
0\end{array}$ & $\begin{array}{l}0 \\
0\end{array}$ & $\begin{array}{l}0 \\
0\end{array}$ & $\begin{array}{l}0 \\
0 \\
0\end{array}$ & 0 \\
\hline $\begin{array}{l}\text { 2-Methylhexane } \\
\text { 2-toxate }\end{array}$ & 0 & 0 & 0 & 0 & 0 & 0 & 0 & 0 & 0 & 0 & 0 & 0 & 0 & 0 & 0 & 0 & 0 & 0 & 0 & 0 & 0 & 0 & 0 & 0 \\
\hline 3-vivenymexane & 0 & 0 & 0 & 0 & 0 & 0 & 0 & 0 & 0 & 0 & 0 & 0 & 0 & 0 & 0 & 0 & 0 & 0 & 0 & 0 & 0 & 0 & 0 & 0 \\
\hline & 0 & 0 & 0 & 0 & 0 & 0 & 0 & 0 & 0 & 0 & 0 & 0 & 0 & 0 & 0 & 0 & 0 & & 0 & 0 & & 0 & 0 & 0 \\
\hline MthCyclohexane & 0 & 0 & 0 & 0 & 0 & 0 & 0 & 0 & 0 & 0 & 0 & 0 & 0 & 0 & 0 & 0 & 0 & 0 & 0 & 0 & 0 & 0 & 0 & 0 \\
\hline 33-2Mth-1-Butene & 0 & 0 & 0 & 0 & 0 & 0 & 0 & 0 & 0 & 0 & 0 & 0 & 0 & 0 & 0 & 0 & 0 & 0 & 0 & 0 & 0 & 0 & 0 & 0 \\
\hline Toluene & 0 & 0 & 0 & 0 & 0 & 0 & & 0 & 0 & 0 & 0 & 0 & 0 & 0 & & 0 & 0 & 0 & 0 & 0 & 0 & 0 & 0 & 0 \\
\hline $0-x y$ & 0 & 0 & 0 & 0 & 0 & 0 & 0 & 0 & 0 & 0 & 0 & 0 & 0 & 0 & 0 & 0 & 0 & 0 & 0 & 0 & 0 & 0 & 0 & 0 \\
\hline M-Xyle & 0 & 0 & 0 & 0 & 0 & 0 & 0 & 0 & 0 & 0 & 0 & 0 & 0 & 0 & 0 & 0 & 0 & 0 & 0 & 0 & 0 & 0 & 0 & 0 \\
\hline e & 0 & 0 & 0 & 0 & 0 & 0 & 0 & 0 & 0 & 0 & 0 & 0 & 0 & 0 & 0 & 0 & 0 & 0 & 0 & 0 & 0 & 0 & 0 & 0 \\
\hline Iluene & 0 & 0 & 0 & 0 & 0 & 0 & 0 & 0 & 0 & 0 & 0 & 0 & 0 & 0 & 0 & 0 & 0 & 0 & 0 & 0 & 0 & 0 & 0 & 0 \\
\hline $\begin{array}{l}\text { 1-2-2-4Trimethylb } \\
\text { 2-MthNaphthalen }\end{array}$ & $\begin{array}{l}0 \\
0 \\
0\end{array}$ & $\begin{array}{l}0 \\
0\end{array}$ & $\begin{array}{l}0 \\
0 \\
0\end{array}$ & $\begin{array}{l}0 \\
0\end{array}$ & $\begin{array}{l}0 \\
0\end{array}$ & $\begin{array}{l}0 \\
0 \\
0\end{array}$ & $\begin{array}{l}0 \\
0 \\
0\end{array}$ & $\begin{array}{l}0 \\
0 \\
0\end{array}$ & $\begin{array}{l}0 \\
0 \\
0\end{array}$ & $\begin{array}{l}0 \\
0 \\
0\end{array}$ & $\begin{array}{l}0 \\
0 \\
0\end{array}$ & $\begin{array}{l}0 \\
0 \\
0\end{array}$ & $\begin{array}{l}0 \\
0\end{array}$ & $\begin{array}{l}0 \\
0 \\
0\end{array}$ & $\begin{array}{l}0 \\
0 \\
0\end{array}$ & $\begin{array}{l}0 \\
0 \\
0\end{array}$ & $\begin{array}{l}0 \\
0\end{array}$ & $\begin{array}{l}0 \\
0 \\
0\end{array}$ & $\begin{array}{l}0 \\
0\end{array}$ & 0 & $\begin{array}{l}0 \\
0 \\
0\end{array}$ & $\begin{array}{l}0 \\
0 \\
0\end{array}$ & $\begin{array}{l}0 \\
0 \\
0\end{array}$ & 0 \\
\hline $\begin{array}{l}\text { vapthalene } \\
\text { 5-TetMthBz }\end{array}$ & $\begin{array}{l}0 \\
0\end{array}$ & 0 & 0 & o & 0 & 0 & $\begin{array}{l}0 \\
0\end{array}$ & $\begin{array}{l}0 \\
0\end{array}$ & o & $\begin{array}{l}0 \\
0\end{array}$ & $\begin{array}{l}0 \\
0\end{array}$ & 0 & $\begin{array}{l}0 \\
0\end{array}$ & 0 & $\begin{array}{l}0 \\
0\end{array}$ & 0 & 0 & $\begin{array}{l}0 \\
0\end{array}$ & 0 & 0 & $\begin{array}{l}0 \\
0\end{array}$ & & o & 0 \\
\hline & 0 & 0 & 0 & 0 & 0 & 0 & 0 & 0 & 0 & 0 & 0 & 0 & 0 & 0 & 0 & 0 & 0 & 0 & 0 & 0 & 0 & 0 & 0 & 0 \\
\hline & 0 & 0 & 0 & 0 & 0 & 0 & 0 & 0 & 0 & 0 & 0 & 0 & 0 & 0 & 0 & 0 & 0 & 0 & & 0 & 0 & & & \\
\hline $3-\mathrm{N}$ & 0 & 0 & 0 & 0 & 0 & 0 & 0 & 0 & 0 & 0 & 0 & 0 & 0 & 0 & 0 & 0 & 0 & 0 & 0 & 0 & 0 & 0 & 0 & 0 \\
\hline texane & 0 & 0 & 0 & 0 & 0 & 0 & 0 & 0 & 0 & 0 & 0 & 0 & 0 & 0 & 0 & 0 & 0 & 0 & 0 & 0 & 0 & 0 & 0 & 0 \\
\hline nth-C5 & 0 & 0 & 0 & 0 & 0 & 0 & 0 & 0 & 0 & 0 & 0 & 0 & 0 & 0 & 0 & 0 & 0 & 0 & 0 & 0 & 0 & 0 & 0 & 0 \\
\hline 1-2-3-4-Tetramet & 0 & 0 & 0 & 0 & 0 & 0 & 0 & 0 & 0 & 0 & 0 & 0 & 0 & 0 & 0 & 8 & 0 & 0 & 0 & 0 & 0 & 0 & 0 & 0 \\
\hline & & & & & & & & & & & & & & & & & & & & & & & & \\
\hline
\end{tabular}




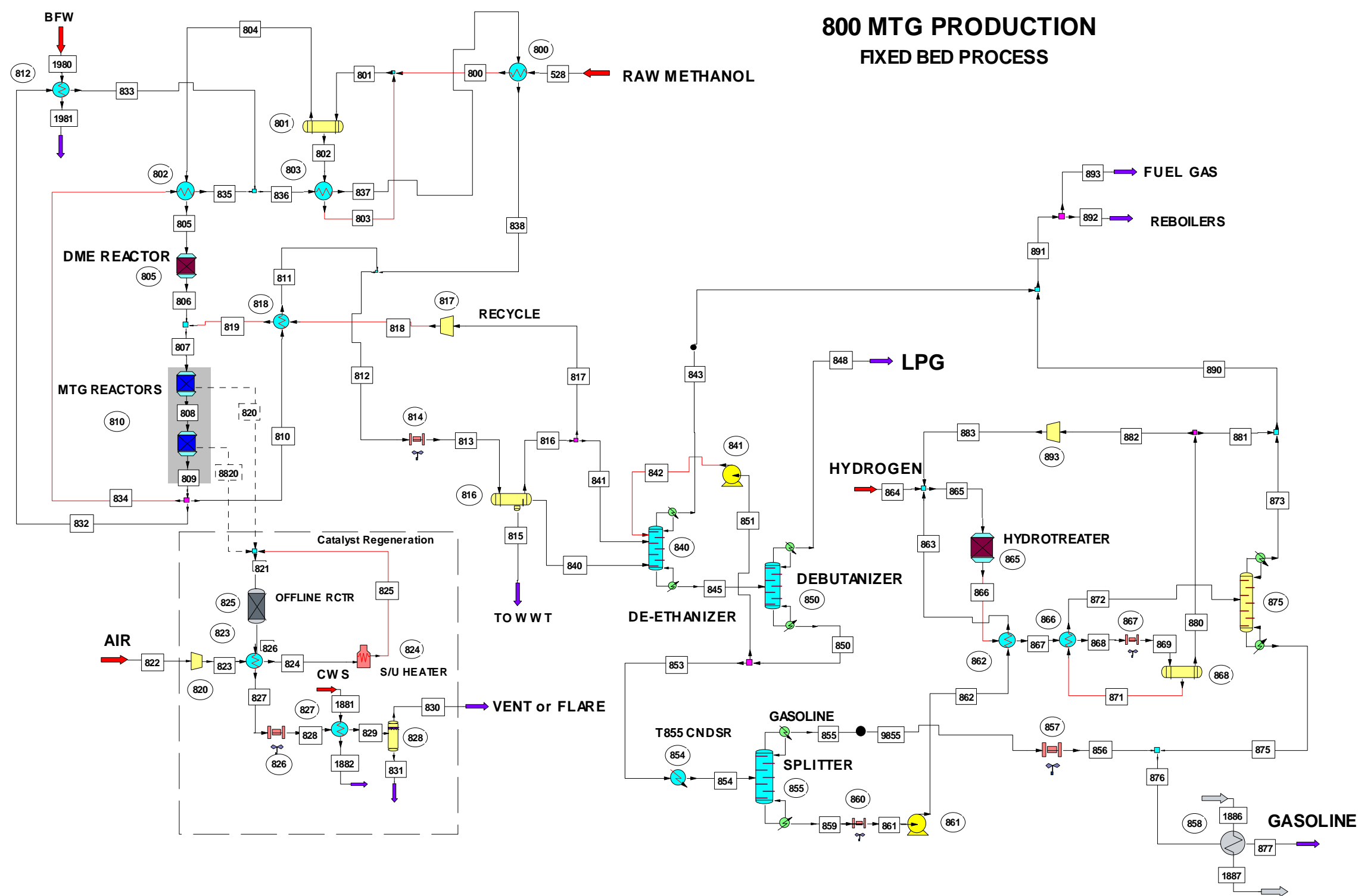

Figure A-2 Process Flow Diagram for the Indirectly-heated Gasifier Case - Gasoline Production 
Table A-2 Steam Results for the Indirectly-heated Gasifier Case - Gasoline Production

\begin{tabular}{|c|c|c|c|c|c|c|c|c|c|c|c|c|c|c|c|c|c|c|c|c|c|c|c|c|c|}
\hline Stream No. & 528 & 800 & 801 & 802 & 803 & 804 & 805 & 806 & 807 & 808 & 809 & 810 & 811 & 812 & 813 & 815 & 816 & 17 & 18 & 19 & 820 & 321 & 822 & 823 & 824 \\
\hline Temp $F$ & 102.597 & 300 & 353.238 & 353.238 & 357.819 & 353.238 & 600 & 756.866 & 616.374 & 622.54 & 734.055 & 734.055 & 212.227 & 236.964 & 110 & 110 & & & & & 622.54 & & 70 & 102.76 & 572 \\
\hline Pres psia & 414.696 & 414.696 & 400 & & & 400 & 400 & & 385 & 330 & 300 & 300 & 300 & 300 & 300 & & & 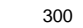 & & 4 & 330 & & 15 & 17 & 16 \\
\hline $\begin{array}{l}\text { Enth MMBtu/h } \\
\text { Vapor mass fraction }\end{array}$ & $\begin{array}{r}-313.98 \\
0\end{array}$ & $\begin{array}{r}-299.35 \\
0\end{array}$ & -596.64 & -332.59 & $\begin{array}{l}-297.29 \\
0\end{array}$ & 264.05 & 253.32 & 253.32 & -2187.1 & -2187.1 & -2187.1 & -1312.3 & $\begin{array}{r}-1454.4 \\
\end{array}$ & $\begin{array}{r}-2401.2 \\
0\end{array}$ & -2484.5 & -360.66 & -2087.9 & -2084.4 & -2074.3 & -1933 & 0.00272 & 1.4456 & 983 & 954 & 1.4001 \\
\hline Total lb/h & 95674.4 & $\begin{array}{l}0.00520 \\
956744\end{array}$ & $\begin{array}{l}0.405921 \\
205921\end{array}$ & 110247 & 110247 & 956742 & 56742 & 956742 & $\begin{array}{r}1 \\
874486\end{array}$ & $\begin{array}{r}1 \\
874466\end{array}$ & 874231 & $\begin{array}{r}1 \\
524538\end{array}$ & 0.96187 & 0.98046 & $\begin{array}{r}0.892 \\
887204\end{array}$ & & 19830 & & & & & & & & \\
\hline wrates in $\mid b / h$ & & & & & & & & & & & & & & 874246 & 874246 & 55.3 & 9830 & 8514 & 78514 & & 56 & & & & \\
\hline than & 337.9 & 37.9 & 1024 & 109387 & 38 & 7.4 & 374 & $3.7 .7-x-3 x-1$ & 63.75 & & & & & 0.0001 & 0.00 & & 0.0001 & 0.0001 & 0.0001 & 0.0002 & 0 & 0 & 0 & 0 & 0 \\
\hline ater & 2256.1 & 2256.1 & 2990.35 & 734.015 & 734.015 & 56.34 & 56.34 & 25441 & 27372.9 & 32486 & 55111.8 & 33067.1 & 33067.1 & 55111.9 & 55111.9 & 53155.3 & 1935.21 & 1931.95 & 1931.95 & 1931.95 & 0 & 0 & & & \\
\hline arbon & & & & & & & & & & & & & & & & & & & & & 19.1156 & 55 & & & \\
\hline drogen & 4.6855 & 4.6855 & 4.8142 & 0.1288 & 0.1288 & 4.6855 & 4.6855 & 4.6855 & 3491.22 & 3491.83 & 3495.45 & 2097.27 & 2097.27 & 3495.37 & 3495.37 & 0 & 3493.9 & 3488 & 3488 & 3487.26 & & & & & \\
\hline rbon Dioxide & 1466.74 & 1466.74 & 1583.81 & 117.075 & 117.075 & 1466.74 & 1466.74 & 1466.74 & 413241 & 413289 & 413972 & 248383 & 248383 & 413996 & 413996 & 0 & 411974 & 411279 & 411279 & 411523 & 0 & 0 & & & \\
\hline than & 4.972 & 304.972 & 3.874 & 8.9015 & 8.9015 & 304.972 & 304.972 & 304.972 & 152155 & 152193 & 152558 & 91534.6 & 91534.6 & 152550 & 152550 & 0 & 152260 & 152003 & 152003 & 151928 & 0 & 0 & & & \\
\hline vyle & 2286 & 286 & .0306 & 0.0021 & 0.0021 & 0.0286 & 0.0286 & 0.0286 & 2097.63 & 2099.54 & 2117.74 & 1270.65 & 1270.65 & 2117.61 & 2117.61 & 0 & 2103.85 & 2100.3 & 2100.3 & 2098.95 & 0 & 0 & & & \\
\hline Ett & 0.0749 & 0.0749 & 0.08 & 0.0051 & 0.0051 & 0.0749 & 0.0749 & 0.0749 & 4195.51 & 4200.28 & 4245.82 & 2547.49 & 2547.49 & 4245.6 & 424 & 0 & 4206.86 & 4199.77 & & 4197.61 & 0 & 0 & & & \\
\hline & & & & & & & & & 748.213 & 750.129 & 768.33 & 460.998 & 460.998 & 768.311 & 768.311 & 0 & & 748.583 & 748.583 & 748.397 & 0 & 0 & & & \\
\hline paa & 0 & 0 & 0 & 0 & 0 & 0 & 0 & 0 & 62752.3 & 62933.8 & 64664 & 38798.4 & 38798.4 & 64662.6 & 64662.6 & 0 & 62885.7 & 62779.6 & 62779.6 & 62765.9 & 0 & 0 & & 0 & \\
\hline $\begin{array}{l}\text { I-Butane } \\
\text { N-Butan }\end{array}$ & $\begin{array}{l}0 \\
0 \\
0\end{array}$ & 0 & 0 & 0 & 0 & 0 & 0 & 0 & 55061.6 & 55396.1 & $\begin{array}{l}58583.3 \\
15263.3\end{array}$ & $\begin{array}{r}35150 \\
0115857\end{array}$ & 35150 & 58582.7 & 58582.7 & 0 & 55165.2 & 55072.1 & 55072.1 & 55066.6 & & & & 0 & \\
\hline Cis-2-2-E & 0 & 0 & 0 & 0 & 0 & 0 & 0 & 0 & $\begin{array}{r}14056.9 \\
7594.5\end{array}$ & $\begin{array}{l}14111.5 \\
7666.17\end{array}$ & $\begin{array}{l}\begin{array}{l}15264.3 \\
8349.12\end{array} \\
8\end{array}$ & $\begin{array}{l}9158.57 \\
50094\end{array}$ & $\begin{array}{l}9158.57 \\
50094\end{array}$ & $\begin{array}{ll}15264.2 \\
834907\end{array}$ & $\begin{array}{ll}15264.2 \\
83407\end{array}$ & $\begin{array}{l}0 \\
0\end{array}$ & $\begin{array}{l}14082.3 \\
760804\end{array}$ & $\begin{array}{l}14058.5 \\
75952\end{array}$ & $\begin{array}{l}14058.5 \\
7595.21\end{array}$ & $\begin{array}{l}14057.6 \\
7598.81\end{array}$ & & & & & \\
\hline nta & 0 & 0 & 0 & 0 & 0 & 0 & 0 & & 21527.8 & 21910.1 & 255526 & 15331.6 & 15331.6 & 255526 & 25552.6 & 0 & 21563.6 & 21527.2 & 21527.2 & 1594 & & & & & \\
\hline enta & 0 & 0 & 0 & 0 & 0 & 0 & 0 & & 4678.84 & 4783.95 & 5785.64 & 3471.39 & 3471.39 & 5785.65 & 5785.65 & 0 & 4686.53 & 4678.62 & 4678.62 & 4678 & & & & & \\
\hline ntene & 0 & 0 & 0 & 0 & 0 & 0 & 0 & 0 & 5 & 5293.98 & 6386.74 & 3832.04 & 3832.04 & 6386.74 & 6386.74 & 0 & 5178.21 & 5169.47 & 5169.47 & 5169.54 & & & & & \\
\hline Cyclopentane & 0 & 0 & 0 & 0 & 0 & 0 & 0 & 0 & 554.7 & 573.815 & 755.951 & 453.571 & 453.571 & 755.952 & 755.952 & 0 & 555.613 & 554.675 & 554.675 & 554.686 & & & & & \\
\hline 2-2-DiM & 0 & 0 & 0 & 0 & 0 & 0 & 0 & & 5056.27 & 5223.49 & 6817.12 & 4090.27 & 4090.27 & 6817.13 & 6817.13 & 0 & 5064.5 & 5055.96 & 5055.96 & 5056.1 & & & & & \\
\hline & 0 & 0 & 0 & 0 & 0 & 0 & 0 & 0 & 3870 & 4037.22 & 5630.85 & 3378.51 & 3378.51 & 5630.86 & 5630.86 & 0 & 3876.32 & 3869.78 & 3869.78 & 3869.89 & & & & & \\
\hline & 0 & 0 & 0 & 0 & 0 & 0 & 0 & & 3622.86 & 3790.08 & 5383.71 & 3230.23 & 3230.23 & 5383.72 & & 0 & & & & 362 & & & & & \\
\hline & 0 & 0 & 0 & 0 & 0 & 0 & 0 & & 3302.68 & 3469.9 & 5063.53 & 3038.12 & 3038.12 & 5063.54 & & & & & & 3 & & & & & \\
\hline & 0 & 0 & 0 & 0 & 0 & 0 & 0 & & 922.963 & 1018.52 & 1564.93 & & 938.958 & 1564.93 & & 0 & & & & & & & & & \\
\hline & 0 & 0 & 0 & 0 & 0 & 0 & 0 & 0 & & 554.642 & 913.401 & 548.04 & 548.04 & 913.401 & & 0 & & & & & & & & & \\
\hline C) & 0 & 0 & 0 & 0 & 0 & 0 & 0 & 0 & $\begin{array}{l}820.354 \\
445.954\end{array}$ & $\begin{array}{l}892.024 \\
\end{array}$ & $\begin{array}{l}1574.97 \\
85807\end{array}$ & $\begin{array}{l}944.985 \\
5148\end{array}$ & $\begin{array}{l}944.985 \\
514\end{array}$ & $\begin{array}{l}1574.98 \\
85800\end{array}$ & & & & $\begin{array}{l}820.323 \\
445.94\end{array}$ & & & & & & $\begin{array}{l}0 \\
0\end{array}$ & \\
\hline Cy & 0 & 0 & 0 & 0 & $\begin{array}{l}0 \\
0\end{array}$ & $\begin{array}{l}0 \\
0\end{array}$ & $\begin{array}{l}0 \\
0\end{array}$ & & $\begin{array}{l}445.954 \\
596.651\end{array}$ & $\begin{array}{l}493.734 \\
6\end{array}$ & $\begin{array}{l}858.006 \\
1351\end{array}$ & $\begin{array}{l}514.803 \\
8\end{array}$ & $\begin{array}{r}514.803 \\
8\end{array}$ & $\begin{array}{l}858.006 \\
1351.2\end{array}$ & $\begin{array}{l}858.006 \\
\end{array}$ & & 699 & $\begin{array}{l}445.945 \\
5\end{array}$ & $\begin{array}{l}945 \\
632\end{array}$ & & & & & & \\
\hline & 0 & 0 & 0 & 0 & 0 & 0 & 0 & 0 & 563.006 & 634.676 & 1317.63 & $\begin{array}{l}710.1100 \\
7990.576\end{array}$ & $\begin{array}{l}\quad 10.100 \\
7990.576\end{array}$ & & & 0 & & $\begin{array}{r}596 \\
56\end{array}$ & $\begin{array}{r}596.6622 \\
562.99\end{array}$ & & & & & & \\
\hline & 0 & 0 & 0 & 0 & 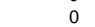 & 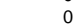 & 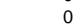 & & 453.807 & 525.477 & 1208.43 & 725.057 & 725.057 & 1208.43 & & 0 & 454.56 & 453.793 & 453.793 & 453.804 & & & & & \\
\hline & 0 & 0 & 0 & 0 & 0 & 0 & 0 & & 377.087 & 472.646 & 1019.05 & 611.433 & 611.433 & 1019.05 & 1019.05 & & 377.717 & 377.08 & 377.08 & & & & & & \\
\hline 33 & 0 & 0 & 0 & 0 & 0 & 0 & 0 & 0 & 2895.59 & 2981.59 & 3710.08 & 2226.05 & 2226.05 & 3710.08 & 3710.08 & & 2900.34 & 2895.45 & 2895.45 & 289 & & & & & \\
\hline & 0 & 0 & 0 & 0 & 0 & 0 & 0 & & & & & 2228.48 & 2228.48 & 3714.14 & & 0 & 1218 & & & & & & & & \\
\hline rimth-C & 0 & 0 & 0 & 0 & 0 & 0 & 0 & 0 & 18 & 23 & 687.664 & 412.598 & 412.598 & 687.664 & & & 184.851 & & 184.539 & & & & & & \\
\hline & 0 & 0 & 0 & 0 & 0 & 0 & 0 & 0 & & 21 & 671.302 & & 402.781 & 671.302 & & 0 & 168.462 & & & & & & & & \\
\hline & 0 & 0 & 0 & 0 & 0 & 0 & 0 & 0 & & & 650 & & & 650 & & 0 & & & & & & & & & \\
\hline & 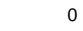 & 0 & 0 & 0 & 0 & 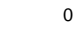 & 0 & & & & & & & & & & & & & & & & & & \\
\hline & 0 & 0 & 0 & 0 & 0 & 0 & 0 & 0 & & & & & & & & & & & & & & & & & \\
\hline & 0 & 0 & 0 & 0 & 0 & 0 & 0 & 0 & & & & & & 268 & & & & & & & & 0 & & & \\
\hline & & 0 & & 0 & 0 & 0 & 0 & 0 & & & & & 646 & & & & & & & & & & & & \\
\hline M-Ethylt & 0 & 0 & 0 & $\begin{array}{l}0 \\
0\end{array}$ & 0 & 0 & 0 & $\begin{array}{l}0 \\
0\end{array}$ & $\begin{array}{l}37.6582 \\
926652\end{array}$ & $\begin{array}{r}88.0496 \\
\end{array}$ & $\begin{array}{r}525.603 \\
155903\end{array}$ & $\begin{array}{r}315.362 \\
935444\end{array}$ & $\begin{array}{r}315.362 \\
935444\end{array}$ & $\begin{array}{r}525.602 \\
1559\end{array}$ & & 0 & & & & & & & & & \\
\hline & 0 & 0 & 0 & 0 & 0 & 0 & $\begin{array}{l}0 \\
0\end{array}$ & $\begin{array}{r}0 \\
59289.1\end{array}$ & $\begin{array}{l}926.667 \\
59289.1\end{array}$ & $\begin{array}{l}1012.67 \\
592891\end{array}$ & $\begin{array}{r}1559.08 \\
\end{array}$ & 46 & 935.446 & $\begin{array}{l}1559.08 \\
\end{array}$ & 15 & & & 92 & & & & & & & \\
\hline & 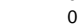 & 0 & 0 & 0 & 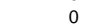 & 0 & 0 & 0 & 73.8279 & $\begin{array}{l}592898.1 \\
214.922\end{array}$ & $\begin{array}{r}0.0013 \\
1440.01\end{array}$ & $\begin{array}{r}0.00008 \\
864.007\end{array}$ & $\begin{array}{r}0.00008 \\
864.007\end{array}$ & $\begin{array}{r}0.0011 \\
1440.01\end{array}$ & $\begin{array}{l}0.6 \\
144\end{array}$ & 0 & $\begin{array}{r}0.0011 \\
739492\end{array}$ & $\begin{array}{r}0.0 \\
73.8\end{array}$ & $\begin{array}{r}0.0 \\
73.8\end{array}$ & & & & & & \\
\hline $1-$ & 0 & 0 & 0 & 0 & 0 & 0 & 0 & 0 & 6.9 & 52.2 & 446 & 267 & 267 & 446 & 44 & 0 & 6.5 & 6.9 & & & & & & ( & \\
\hline & 0 & 0 & 0 & 0 & 0 & 0 & 0 & & 4.137 & 28.595 & 264.862 & 158.917 & 158.917 & 264.862 & 264.862 & 0 & 4.1438 & 4.1 & 4.1368 & 4.1 & 0 & 0 & & 0 & \\
\hline halene & 0 & 0 & 0 & 0 & 0 & 0 & 0 & 0 & 0.1988 & 4.2308 & 39.2114 & 23.5268 & 23.5268 & 39.2113 & 39.2113 & 0 & 0.1991 & 0.1988 & 0.1988 & 0.1988 & 0 & 0 & & & \\
\hline & & & 0 & & 0 & 0 & 0 & & & & & & & & & 0 & & & & & 0 & & & & \\
\hline & 3.9133 & 3.9133 & 4.0058 & 0.0925 & 0.0925 & 3.9133 & 3.9133 & 3.9133 & 2505.6 & 2505.6 & 2505.6 & 1503.36 & 1503.36 & 2505.69 & 2505.69 & 0 & 2504.2 & 2499.98 & 2499.98 & 2500.86 & 0 & 42 & 12 & 42 & 42 \\
\hline 2-3-4-Tetramet & 0 & 0 & 0 & 0 & 0 & 0 & 0 & 0 & 0 & 0 & 0 & 0 & 0 & 0 & 0 & 0 & 0 & 0 & 0 & 0 & 0 & 0 & 0 & 0 & \\
\hline & & & & & & & & & & & & & & & & & & & & & & & & & \\
\hline
\end{tabular}




\begin{tabular}{|c|c|c|c|c|c|c|}
\hline Stream No. & & & & 828 & 829 & 830 \\
\hline & 572 & 1705.94 & 1303.86 & 150 & 110 & 110 \\
\hline Pres psia & & & & 5 & & \\
\hline $\begin{array}{l}\text { Enth MMBtu/h } \\
\text { ntion }\end{array}$ & 1.4001 & 1.4456 & 0.11501 & -3.3604 & -3.4568 & -3.4568 \\
\hline Vapor mass fraction & & & & & & \\
\hline Total lb/h & & 11796.2 & 11796.2 & 11796.2 & 11796.2 & \\
\hline Flowrates in $\mathrm{lb} / \mathrm{h}$ & & & & & & \\
\hline Methanol & 0 & 0 & 0 & 0 & 0 & \\
\hline Water & 0 & & & & & \\
\hline Carbon & 0 & 0 & 0 & 0 & 0 & 0 \\
\hline $\begin{array}{l}\text { Hydrogen } \\
\text { Paroxide }\end{array}$ & $0_{0}^{0}$ & $\begin{array}{r}0 \\
937495\end{array}$ & $\begin{array}{r}0 \\
937495\end{array}$ & $\begin{array}{r}0 \\
02705\end{array}$ & 0 & 0 \\
\hline $\begin{array}{l}\text { Carbon Dioxide } \\
\text { Methane }\end{array}$ & $\begin{array}{l}0 \\
0 \\
0\end{array}$ & 937.495 & 937.495 & 937.495 & 937.495 & 937.495 \\
\hline $\begin{array}{l}\text { Methane } \\
\text { Ethylene }\end{array}$ & $\begin{array}{l}0 \\
0\end{array}$ & $\begin{array}{l}0 \\
0\end{array}$ & $\begin{array}{l}0 \\
0 \\
0\end{array}$ & $\begin{array}{l}0 \\
0 \\
0\end{array}$ & & \\
\hline Ethane & 0 & 0 & 0 & 0 & 0 & 0 \\
\hline Propylene & 0 & 0 & 0 & 0 & 0 & \\
\hline Propane & 0 & 0 & 0 & 0 & 0 & 0 \\
\hline I-Butane & 0 & 0 & 0 & 0 & 0 & 0 \\
\hline N-Butane & 0 & 0 & 0 & 0 & 0 & 0 \\
\hline Cis-2-Butene & 0 & 0 & 0 & 0 & 0 & 0 \\
\hline I-Pentane & 0 & 0 & 0 & 0 & 0 & 0 \\
\hline N-Pentane & 0 & 0 & 0 & 0 & 0 & 0 \\
\hline Trans-2-Pentene & 0 & 0 & 0 & 0 & 0 & 0 \\
\hline Cyclopentane & 0 & 0 & 0 & 0 & 0 & 0 \\
\hline 2-2-DiMth-Butane & 0 & 0 & 0 & 0 & 0 & 0 \\
\hline $\begin{array}{l}\text {-2-3-DiMth-Butane } \\
\text { 2-Methylpentane }\end{array}$ & $\begin{array}{l}0 \\
0\end{array}$ & $\begin{array}{l}0 \\
0\end{array}$ & $\begin{array}{l}0 \\
0\end{array}$ & $\begin{array}{l}0 \\
0\end{array}$ & $\begin{array}{l}0 \\
0\end{array}$ & 0 \\
\hline 3-Methylpentane & 0 & 0 & 0 & & & 0 \\
\hline MthCyclopentane & 0 & 0 & 0 & 0 & 0 & 0 \\
\hline Benzene & 0 & 0 & 0 & 0 & 0 & 0 \\
\hline 2-4-DiMthPentane & 0 & 0 & 0 & 0 & 0 & 0 \\
\hline Cyclohexane & 0 & 0 & 0 & 0 & 0 & 0 \\
\hline 2-Methylhexane & 0 & 0 & 0 & 0 & 0 & 0 \\
\hline 3-Methylhexane & 0 & 0 & 0 & 0 & 0 & 0 \\
\hline 2-2-4-TriMth-C5 & 0 & 0 & 0 & 0 & 0 & 0 \\
\hline MthCyclohexane & 0 & 0 & 0 & 0 & 0 & o \\
\hline 33-2Mth-1-Butene & 0 & 0 & 0 & 0 & 0 & o \\
\hline $\begin{array}{l}\text { Toluene } \\
2-3-3-T \text { TiMth-C5 }\end{array}$ & 0 & 0 & 0 & 0 & 0 & 0 \\
\hline $\begin{array}{l}\text {-3-3-3-Tilith-C5 } \\
\text { 2-3-DiMth-Hexane }\end{array}$ & $\begin{array}{l}0 \\
0 \\
0\end{array}$ & $\begin{array}{l}0 \\
0\end{array}$ & 0 & 0 & 0 & 0 \\
\hline $\begin{array}{l}\text {-3-3-DiMth-Hexane } \\
\text { 3-Methylheptane }\end{array}$ & $\begin{array}{l}0 \\
0 \\
0\end{array}$ & $\begin{array}{l}0 \\
0\end{array}$ & $\begin{array}{l}0 \\
0\end{array}$ & $\begin{array}{l}0 \\
0 \\
0\end{array}$ & $\begin{array}{l}0 \\
0 \\
0\end{array}$ & 0 \\
\hline 23-2Mth-1-Butene & 0 & 0 & 0 & 0 & 0 & 0 \\
\hline P-Xylene & 0 & 0 & 0 & 0 & 0 & 0 \\
\hline M-Xylene & 0 & 0 & 0 & 0 & 0 & 0 \\
\hline O-Xylene & 0 & 0 & 0 & 0 & 0 & 0 \\
\hline M-Ethyltoluene & 0 & 0 & 0 & 0 & 0 & 0 \\
\hline 23-2Mth-2-Butene & 0 & 0 & 0 & 0 & 0 & 0 \\
\hline Dimethyl Ether & 0 & 0 & 0 & 0 & 0 & 0 \\
\hline 1-2-4-Trimethylb & 0 & 0 & 0 & 0 & 0 & 0 \\
\hline 1-2-4-5-TetMthBz & 0 & 0 & 0 & 0 & 0 & 0 \\
\hline Naphthalene & 0 & 0 & 0 & 0 & 0 & 0 \\
\hline 2-MthNaphthalene & $\begin{array}{r}0 \\
069702\end{array}$ & 0 & 0 & 0 & 0 & \\
\hline $\begin{array}{l}\text { Oxygen } \\
\text { Nitrogen }\end{array}$ & $\begin{array}{l}2687.92 \\
20852.02\end{array}$ & 2006.28 & 2006.28 & 2006.28 & 2006.28 & 2006.28 \\
\hline $\begin{array}{l}\text { Nitrogen } \\
\text { 1-2-3-4-Tetramet }\end{array}$ & 8852.42 & 8852.42 & 8852.42 & 8852.42 & 8852.42 & 8852.42 \\
\hline 1-1-Dichloro-2-2 & 0 & 0 & 0 & 0 & 0 & 0 \\
\hline
\end{tabular}

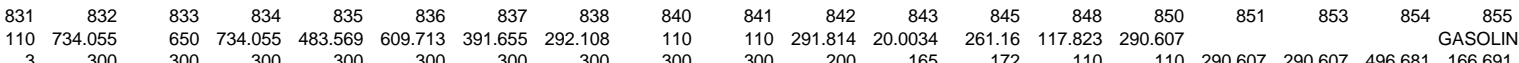

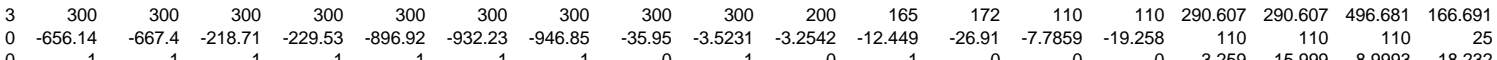

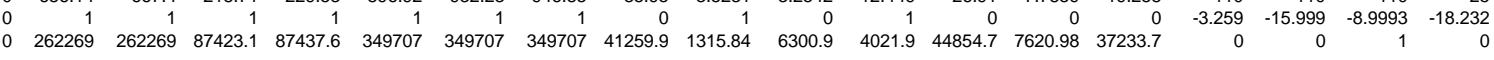

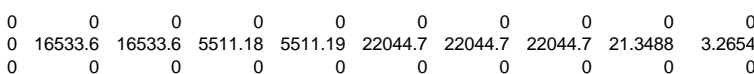

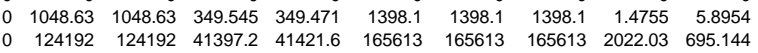

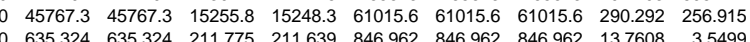
$\begin{array}{llllllllll}0 & 0.5375\end{array}$ $\begin{array}{llllllllll}0 & 230.499 & 230.499 & 76.833 & 76.814 & 307.313 & 307.313 & 307.313 & 18.4632 & 1.2653 \\ 0 & 19399.2 & 193992 & 6466.4 & 6464.98 & 25864.2 & 258642 & 258642 & 1776.85 & 106.11\end{array}$ $\begin{array}{rrrrr}0 & 0 & 0 & 0 & 0 \\ 0 & 24.6142 & 0 & 0 & 0 \\ 0 & 0 & 0 & 0 & 0 \\ 0 & 7.3709 & 0 & 0 & 0 \\ 0 & 2717.17 & 0 & 0 & 0 \\ 0 & 547.207 & 0 & 0 & 0 \\ 0 & 17.3108 & 0 & 0 & 0 \\ 0 & 45.8336 & 0 & 0 & 0 \\ 0 & 9.3163 & 10.4121 & 10.4122 & 0\end{array}$

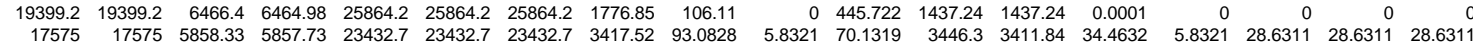

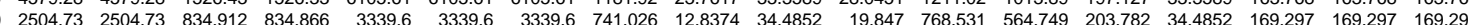
$\begin{array}{lllllllllllllllllll}0 & 7665.79 & 7665.79 & 2555.26 & 2555.27 & 10221.1 & 10221.1 & 10221.1 & 3989.05 & 36.3853 & 608.064 & 46.3767 & 4587.17 & 993.957 & 3593.21 & 608.064 & 2985.15 & 2985.15 & 2985.15\end{array}$ $\begin{array}{lllllllllllllllllll}0 & 1735.69 & 1735.69 & 578.564 & 578.572 & 2314.26 & 2314.26 & 2314.26 & 1099.12 & 7.9078 & 210.467 & 8.3276 & 1309.16 & 65.4574 & 1243.7 & 210.467 & 1033.24 & 1033.24 & 1033.24\end{array}$

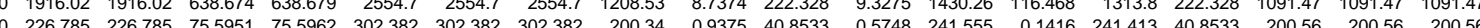

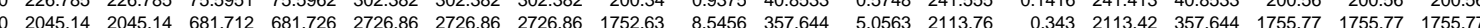

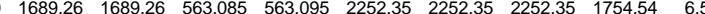

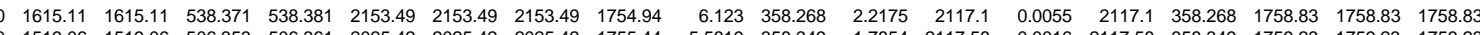

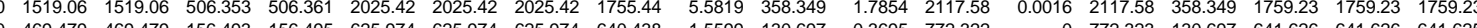

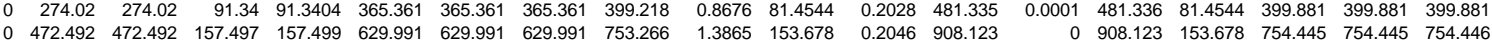

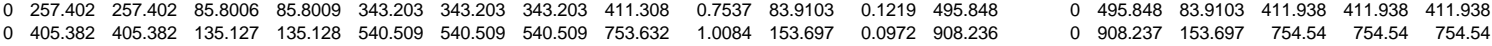
$\begin{array}{lllllllllll}0 & \end{array}$ $\begin{array}{lllllllllllll}0 & 395.288 & 395.288 & 131.763 & 131.763 & 527.051 & 527.051 & 527.051 & 753.686 & 0.9516 & 153.699 & 0.0855 & 908.247 \\ 0 & 362528 & 362528 & 120843 & 120843 & 483731 & 48.371 & 483.371 & 753868 & 0.767 & 153705 & 0.0519 & 908284\end{array}$ $\begin{array}{lllllllllllll}0 & 305.716 & 305.716 & 101.905 & 101.905 & 407.622 & 407.622 & 407.622 & 641.337 & 0.6373 & 130.758 & 0.0466 & 772.682\end{array}$ $\begin{array}{lllllllllllll}0 & 1113.02 & 1113.02 & 371.008 & 371.013 & 1484.04 & 1484.04 & 1484.04 & 809.74 & 4.8939 & 163.783 & 4.1292 & 974.283 \\ 0 & 111424 & 111424 & 371.414 & 371.412 & 1485.65 & 1485.65 & 1485.65 & 2496.14 & 2.0552 & 508.843 & 0.1362 & 3006.89\end{array}$ $\begin{array}{lllllllllllll}0 & 1114.24 & 1114.24 & 371.414 & 371.412 & 1485.65 & 1485.65 & 1485.65 & 2496.14 & 2.0552 & 508.843 & 0.1362 & 3006.89 \\ 0 & 206.299 & 206.299 & 68.7664 & 68.7662 & 275065 & 275065 & 275065 & 502813 & 03119 & 102.482 & 0.016 & 605.592\end{array}$

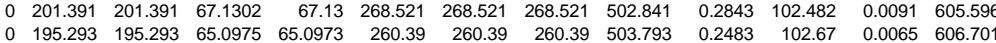
$\begin{array}{lllllllllrlrl}0 & 813.105 & 813.105 & 271.035 & 271.039 & 1084.14 & 1084.14 & 1084.14 & 811.38 & 3.2042 & 165.629 & 1.4445 & 978.766 \\ 0 & 368.094 & 368.094 & 122.698 & 122.697 & 490.792 & 490.792 & 490.792 & 1037.34 & 0.32 & 211.365 & 0.0061 & 1249.01\end{array}$

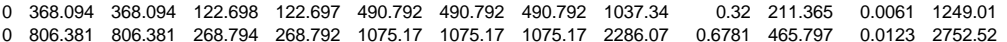

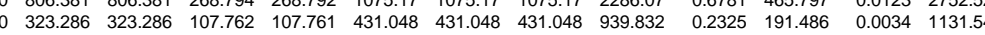
$\begin{array}{lllllllllllll}0 & 157.681 & 157.681 & 52.5603 & 52.5598 & 210.241 & 210.241 & 210.241 & 487.882 & 0.0636 & 99.3922 & 0.0004 & 587.335\end{array}$ $\begin{array}{lllllllllllll}0 & 467.723 & 467.723 & 155.908 & 155.91 & 623.632 & 623.632 & 623.632 & 630.882 & 1.5662 & 128.752 & 0.3667 & 760.83\end{array}$

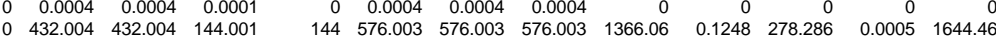

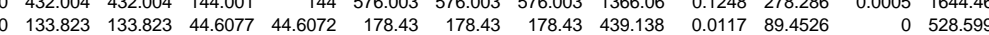
$\begin{array}{rrrrrrrrrrrrr}0 & 79.4586 & 79.4586 & 26.4862 & 26.4859 & 105.945 & 105.945 & 105.945 & 260.718 & 0.007 & 53.1084 & 0 & 313.832 \\ 0 & 11.7634 & 11.7634 & 3.9211 & 3.9211 & 15.6845 & 15.6845 & 15.6845 & 39.0122 & 0.0003 & 7.9467 & 0 & 46.959\end{array}$ $\begin{array}{rrrrrrrrrr}0 & 0 & 0 & 0 & 0 & 0 & 0 & 0 & 0 & 0 \\ 0 & 751.68 & 751.68 & 250.56 & 250.649 & 1002.33 & 1002.33 & 1002.33 & 1.4843 & 4.2255 \\ 0 & 0 & 0 & 0 & 0 & 0 & 0 & 0 & 0 & 0 \\ 0 & 0 & 0 & 0 & 0 & 0 & 0 & 0 & 0 & 0\end{array}$ $\begin{array}{llllll}9 & 908.247 & 153.699 & 754.549 & 754.549 & 754.549 \\ 0 & 908.284 & 153.705 & 754.579 & 754.579 & 754579\end{array}$ $0 \quad 772.682 \quad 130.758 \quad 641.924 \quad 641.924 \quad 641.924$

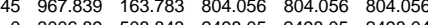

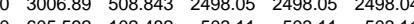
O $605.596 \quad 102.482503 .113 \quad 503.113 \quad 503.113$ $0 \quad 606.702 \quad 102.67 \quad 504.032 \quad 504.032 \quad 504.032$

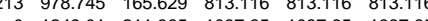

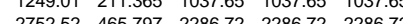
$1131.54 \quad 191.486 \quad 940.056$
0 $\begin{array}{llllll}0 & 587.335 & 99.3922 & 487.943 & 487.943 & 487.941 \\ 0 & 760.831 & 128.752 & 632.079 & 632.079 & 632.078\end{array}$ $\begin{array}{rrrrrr}0 & 0 & 0 & 0 & 0 & 0 \\ 0 & 1644.46 & 278.286 & 1366.18 & 1366.18 & 1366.17\end{array}$ $\begin{array}{llllll}0 & 313.832 & 53.1084 & 260.723 & 260.723 & 0.0005 \\ 0 & 46.959 & 7.9467 & 39.0123 & 39.0123 & 0\end{array}$ 1-1-Dichloro-2-2

\begin{tabular}{lllllll}
.92 & 2086.28 & 2006.28 & 2006.28 & 2006.28 & 2006.28 \\
\hline & 8852.42 & 8852.42 & 8852.42 & 8852.42 & 8852.42
\end{tabular}

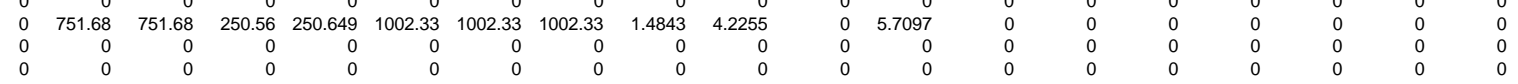




\begin{tabular}{|c|c|c|c|c|c|c|c|c|c|c|c|c|c|c|c|c|c|c|c|c|c|c|c|c|c|}
\hline $\begin{array}{l}\text { Stream No. } \\
\text { Stream Name }\end{array}$ & 856 & 859 & 861 & 862 & 863 & $\begin{array}{c}864 \\
\text { HYDROG }\end{array}$ & EN 865 & 866 & 867 & 868 & 869 & 871 & $\begin{array}{l}872 \\
200\end{array}$ & $\begin{array}{r}873 \\
231.548\end{array}$ & $\begin{array}{r}875 \\
550.77\end{array}$ & $\begin{array}{r}876 \\
159.571\end{array}$ & $\begin{array}{l}877 \\
110\end{array}$ & $\begin{array}{l}880 \\
150\end{array}$ & $\begin{array}{l}881 \\
150\end{array}$ & $\begin{array}{l}882 \\
150\end{array}$ & $\begin{array}{r}883 \\
177.321\end{array}$ & $\begin{array}{r}890 \\
149.652\end{array}$ & $\begin{array}{r}891 \\
41.6806\end{array}$ & $\begin{array}{r}892 \\
41.6806\end{array}$ & $\begin{array}{r}893 \\
41.6806\end{array}$ \\
\hline Temp F & 149 & 443.08 & 175 & 179.876 & 400 & & & 539.743 & 454.699 & 439.931 & 150 & 150 & & & & & & & $\begin{array}{l}150 \\
450\end{array}$ & & & & & & $\begin{array}{r}41.6800 \\
100\end{array}$ \\
\hline Pres psia & 25 & 25 & 20 & & 470 & 650 & & & & & 450 & 450 & -0.2533 & -0.0049 & -0.09021 & -18.611 & -19.412 & -0.00711 & -0.00356 & -0.00356 & 0.00031 & -0.00845 & -12.457 & & -8.6575 \\
\hline Enth MMBtu/h & -18.52 & 0813 & -0.01431 & -0.01276 & 0.06451 & -0.00165 & 0.06317 & 0.06317 & -0.0141 & -0.03146 & -0.27778 & -0.27066 & & & & & & & & & & & 0.9944 & 0.9944 & 0.9944 \\
\hline Vapor mass fraction & & & 0 & & & & 0.16551 & & 0.76498 & 0.67917 & 0.12062 & & 727.507 & 6.1455 & 721.362 & 30937.3 & 30937.3 & 99.7881 & 49.894 & 49.894 & 49.894 & 56.0396 & 4077.94 & 1243.85 & 2834.09 \\
\hline Methanol & 0 & 0 & 0 & 0 & 0 & 0 & 0 & 0 & 0 & 0 & 0 & 0 & 0 & & & 0 & 0 & 0 & 0 & 0 & 0 & 0 & & 0 & \\
\hline Water & & 0 & 0 & & 0 & 0 & 0 & & & & & & & & & & & & & & & & 6142 & 7.5078 & \\
\hline Carbon & & 0 & 0 & 0 & 0 & 0 & 0 & 0 & 0 & 0 & 0 & 0 & 0 & 0 & 0 & & 0 & 0 & 0 & 0 & 0 & 0 & & & $\begin{array}{l}1004 \\
0\end{array}$ \\
\hline arog & & 0 & 0 & 0 & 0 & .474 & 101.522 & 276 & 281 & 281 & 281 & 1859 & 1859 & 1855 & 0.0004 & 0.0004 & 0004 & 82.0951 & 0476 & 41.0476 & 11.0476 & 41.2331 & 18.604 & 51 & 37788 \\
\hline ide & & 0 & 0 & 0 & 0 & 0 & & & & & & & & & & & & & & & & & 2717.17 & $\begin{array}{l}148.789 \\
8289\end{array}$ & $\begin{array}{l}1888.38 \\
\end{array}$ \\
\hline thar & & & 0 & 0 & 0 & 0 & 0 & 0 & 0 & & & & & & & & & & & & & 0 & & 166909 & $\begin{array}{l}1000.000 \\
380299\end{array}$ \\
\hline thylene & 0 & 0 & 0 & 0 & 0 & 0 & 0 & 0 & 0 & 0 & 0 & 0 & 0 & 0 & 0 & 0 & 0 & 0 & 0 & 0 & 0 & 0 & 17.3108 & 5.2801 & 12.0307 \\
\hline nane & 0 & 0 & 0 & 0 & 0 & 0 & 0.4104 & 0.859 & 0.8589 & 0.8589 & 0.8589 & 0.0381 & 0.0381 & 0.0377 & 0.0004 & 0.0004 & 0.0004 & 0.8208 & 0.4104 & 0.4104 & 0.4104 & 0.4481 & 46.2817 & 14.1168 & 32.1649 \\
\hline opylene & 0 & 0 & 0 & 0 & 0 & 0 & & & & & & & & & & & & & & & & & 9.3163 & 2.8416 & 6.4746 \\
\hline opane & & 0 & 0 & 0 & 0 & 0 & 2.2553 & 5.1335 & 5.1332 & 5.1332 & 5.1332 & 0.6226 & 0.6224 & 0.6051 & 0.0174 & 0.0174 & 0.0174 & 4.5106 & 2.2553 & 2.2553 & 2.2553 & 2.8603 & 448.582 & 136.826 & 311.756 \\
\hline utane & 6311 & 0 & 0 & 0 & 0 & 0 & 1.7326 & 4.4728 & 4.4727 & 4.4727 & 4.4727 & 1.0075 & 1.0073 & 0.9524 & 0.0549 & 28.686 & 28.686 & 3.4652 & 1.7326 & 1.7326 & 1.7326 & 2.685 & 72.8169 & 22.2105 & 50.6063 \\
\hline Butane & .768 & 0 & 0 & 0 & 0 & 0 & 1.02 & 2.8453 & 2.8453 & 2.8453 & 2.8453 & 0.8054 & 0.8052 & 0.7511 & 0.0541 & 163.822 & 163.822 & 2.0399 & 1.02 & 1.02 & 1.02 & 1.7711 & 29.8161 & 9.0945 & 20.7216 \\
\hline ene & 297 & 0 & 0 & 0 & 0 & 0 & & & & & & & & & & 169.297 & 169.297 & & 0 & 0 & 0 & & 19.847 & 6.0537 & 13.7933 \\
\hline enta & 3.15 & 0 & 0 & 0 & 0 & 0 & 1.2952 & 4.7499 & 4.7499 & 4.7499 & 4.7499 & 2.1595 & 2.1593 & 1.8835 & 0.2758 & 2985.42 & 2985.42 & 2.5905 & 1.2952 & 1.2952 & 1.2952 & 3.1788 & 49.5555 & 15.1154 & 34.4401 \\
\hline Pent & 3.24 & 0 & 0 & 0 & 0 & 0 & & & & & & & & & & 1033.24 & 1033.24 & 0 & & 0 & 0 & & 8.3276 & 2.5401 & 5.7875 \\
\hline ans- 2 & & 0 & 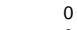 & 0 & 0 & 0 & 0.1038 & 0.4549 & 0.4549 & 0.4549 & 0.4549 & 0.2474 & 0.2474 & 0.2053 & 0.0421 & & 1091.51 & 0.2075 & 0.1038 & 0.1038 & 0.1038 & 0.3091 & & & \\
\hline & & 0 & 0 & 0 & 0 & 0 & & & & & & & & & & & & & & & & 0 & & 0.1753 & 0.3995 \\
\hline & 5577 & 0 & 0 & 0 & 0 & 0 & 0 & 0 & 0 & 0 & & 0 & 0 & 0 & 0 & 1755.77 & 1755.77 & 0 & 0 & 0 & 0 & 0 & 5.0563 & 1.5423 & .5141 \\
\hline & & 0 & 0 & 0 & n & 0 & & & & 0 & & & 0 & 0 & & & 1758.41 & 0 & 0 & 0 & 0 & 0 & 2.6484 & 0.8078 & .8406 \\
\hline $\begin{array}{l}\text { Methylpentane } \\
\text { Methylpontanee }\end{array}$ & 8.83 & 0 & 0 & 0 & 0 & 0 & 0.3563 & 2.1972 & 2.1972 & 2.1972 & 2.1972 & 1.4847 & 1.4847 & 1.0943 & 0.3904 & 1759.22 & 1759.22 & 0.7125 & 0.3563 & 0.3563 & 0.3563 & 1.4506 & & 1188 & 5492 \\
\hline $\begin{array}{l}\text { Methylpentane } \\
\text { hCyclopentane }\end{array}$ & $\begin{array}{l}59.23 \\
625\end{array}$ & $\begin{array}{l}0 \\
0 \\
0\end{array}$ & 0 & 0 & ${ }_{0}^{0}$ & $\begin{array}{l}0 \\
0\end{array}$ & 0 & 0 & 0 & & & & & & 0 & 1759.23 & 1759.23 & & 0 & & 0 & 0 & $\begin{array}{l}1.7854 \\
0.395\end{array}$ & 446 & \\
\hline ксус & $\begin{array}{l}.625 \\
881\end{array}$ & & 0 & $\begin{array}{l}0 \\
0 \\
0\end{array}$ & $\begin{array}{l}0 \\
0 \\
0\end{array}$ & $\begin{array}{l}0 \\
0\end{array}$ & $\begin{array}{l}0 \\
0\end{array}$ & $\begin{array}{l}0 \\
0\end{array}$ & 0 & & & & & & & $\begin{array}{l}641.625 \\
399.881\end{array}$ & $\begin{array}{l}641.626 \\
39988 ?\end{array}$ & & & & & & 0.3695 & 0.1127 & 0.2568 \\
\hline nta & 6 & 0 & 0 & 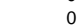 & 0 & 0 & 0 & 0 & 0 & 0 & 0 & 0 & 0 & 0 & 0 & $\begin{array}{l}399.881 \\
754.446\end{array}$ & $\begin{array}{l}395 . \\
754\end{array}$ & & & & & 0 & $\begin{array}{l}0.2028 \\
0.2046\end{array}$ & $\begin{array}{l}0.019 \\
0.0624\end{array}$ & $\begin{array}{l}0.141 \\
0.1422\end{array}$ \\
\hline & 38 & 0 & 0 & 0 & 0 & 0 & 0 & 0 & 0 & 0 & 0 & 0 & 0 & 0 & 0 & 411.938 & 411.938 & & & & & & 0.1219 & & 0.0847 \\
\hline & & 政 & 0 & 0 & 0 & 0 & 0 & 0 & 0 & 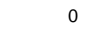 & 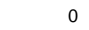 & 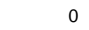 & 0 & 0 & 0 & 754.54 & 754 & & & & & & & & 76 \\
\hline & 19 & 0 & c & 0 & 0 & 0 & 0 & 0 & 0 & & & 0 & 0 & 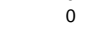 & 0 & 754.549 & 754. & & & & & & & & \\
\hline & 9 & & & & 0 & 0 & 0 & 0 & 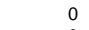 & & & & 0 & , & 0 & 754 & & & & & & & & & 61 \\
\hline & & c & & & & ( & 0 & 0 & 0 & & & & 0 & 0 & 0 & & & & & & & 0 & & & \\
\hline & & & & & & c & 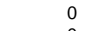 & 0 & & & & & 0 & , & 0 & & & & & & & & & & \\
\hline & & ( & & 0 & 0 & 0 & 0 & 0 & 0 & 0 & & & 0 & 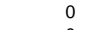 & 0 & & & & & & & 0 & & & 47 \\
\hline & & 0 & c & 0 & 0 & 0 & 0 & 0 & 0 & & & & 0 & 0 & 0 & & & & & & & 0 & & & \\
\hline & & 0 & & & 0 & 0 & 0 & 0 & & & & & & 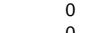 & 0 & & & & & & & 0 & & 28 & 63 \\
\hline & 2 & 0 & & 0 & 0 & 0 & & & & & & & & & 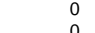 & & & & & & & 0 & & & \\
\hline utene & 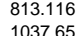 & $\begin{array}{l}0 \\
0 \\
0\end{array}$ & 0 & $\begin{array}{l}0 \\
0\end{array}$ & $\begin{array}{l}0 \\
0 \\
0\end{array}$ & 0 & $\begin{array}{l}0 \\
0 \\
0\end{array}$ & 0 & 0 & 0 & 0 & 0 & 0 & 0 & 0 & $\begin{array}{l}813.116 \\
1037\end{array}$ & 81 & & & 0 & & 0 & & 406 & 0039 \\
\hline Iyle & $\begin{array}{l}5 \\
2 \\
2\end{array}$ & $\begin{array}{l}0 \\
0 \\
0\end{array}$ & $\begin{array}{l}0 \\
0\end{array}$ & $\begin{array}{l}0 \\
0 \\
0\end{array}$ & $\begin{array}{l}0 \\
0 \\
0\end{array}$ & $\begin{array}{l}0 \\
0\end{array}$ & $\begin{array}{l}0 \\
0\end{array}$ & $\begin{array}{l}0 \\
0\end{array}$ & 0 & 0 & 0 & 0 & 0 & $\begin{array}{l}0 \\
0\end{array}$ & $\begin{array}{l}0 \\
0\end{array}$ & 103 & & & & 0 & & 0 & & 19 & 0042 \\
\hline & & & & & & 0 & & & & & & & & 0 & & 94 & & & & & & 0 & & 01 & $\begin{array}{l}83 \\
23 \\
\end{array}$ \\
\hline & .941 & 0.0001 & 0.0001 & 0.0001 & 0.0001 & 0 & 0.0001 & 0.0001 & 0.0001 & 0.0001 & 0.0001 & 0.0001 & 0.0001 & 0 & 0.0001 & 487 & 487 & & & & & 0 & & 0.0001 & 0.0003 \\
\hline & .078 & & & & & 0 & & & & & & & 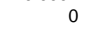 & 0 & 0 & 632 & 632 & & & & & & 67 & 0.1118 & 0.2548 \\
\hline & & & & & & 0 & & & & & & & & 0 & & & & & & & & & & & \\
\hline & & & & & & 0 & & 0.0067 & 0.0067 & 0.0067 & 0.0067 & 0.0066 & 0.0066 & 0 & 66 & & & 0.0001 & & & & 0 & 06 & 0.0002 & .0004 \\
\hline & & & & & & 0 & & & & & & & & & & & & & & & & & & & \\
\hline & 0.0005 & & & & & 0 & 260.93 & 260.93 & 260.93 & 260.93 & 260.93 & 260.516 & 260.516 & 0.0088 & 260.507 & & & 0.4147 & & & & & & & 502 \\
\hline halene & & 39.0123 & 39.0123 & 39.0123 & 39.0123 & 0 & 39.0225 & 39.0225 & 39.0225 & 39.0225 & 39.0225 & 39.0021 & 39.0021 & 0.0001 & 39.002 & 39.002 & 39.002 & 0.0204 & 0.0102 & 0.0102 & 0.0102 & 0.0103 & 0.0103 & 0.0031 & 0.0072 \\
\hline & 0 & 0 & 0 & 0 & 0 & 0 & 0 & & 0 & 0 & 0 & & 0 & 0 & 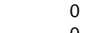 & & & & 0 & 0 & & 0 & & & \\
\hline & 0 & 0 & 0 & 0 & 0 & 0 & & & & & & & & & & & & & & & & & 5.7097 & 1.7416 & 3.9682 \\
\hline $\begin{array}{l}\text {-2-3-4-Tetramet } \\
\text {--Dichloro-2-2 }\end{array}$ & $\begin{array}{l}0 \\
0\end{array}$ & $\begin{array}{l}0 \\
0 \\
0\end{array}$ & 0 & 0 & $\begin{array}{l}0 \\
0 \\
0\end{array}$ & $\begin{array}{l}0 \\
0\end{array}$ & 555 & $\begin{array}{r}.343 \\
0\end{array}$ & 424.343 & $\begin{array}{r}424.343 \\
0\end{array}$ & $\begin{array}{r}424.343 \\
0\end{array}$ & $\begin{array}{r}1.432 \\
0\end{array}$ & $\begin{aligned} 32 \\
0\end{aligned}$ & 0 & .011 & 0 & $\begin{array}{r}1.011 \\
0\end{array}$ & $\begin{array}{r}09 \\
0\end{array}$ & $\begin{aligned} 1.4555 \\
0\end{aligned}$ & $\begin{array}{l}55 \\
0 \\
0\end{array}$ & 0 & $\begin{aligned} 1.8769 \\
0\end{aligned}$ & 1.8769 & 0.5725 & 1.3044 \\
\hline
\end{tabular}




\begin{tabular}{|c|c|c|c|c|c|c|c|c|c|c|c|c|c|c|c|c|}
\hline Stream No. & 1740 & 1741 & 1840 & 1841 & 1881 & 1882 & 1886 & 1887 & 1980 & 1981 & 8820 & 9840 & 9841 & 9842 & 9843 & 9855 \\
\hline Temp F & 2 & 20 & 90 & 110 & 90 & 110 & 90 & 110 & 176 & 525.213 & 734.055 & 165.272 & 164 & 20 & 20.0034 & 166.691 \\
\hline Pres psia & 16 & 16 & 90 & 90 & 90 & 88 & 90 & 85 & 850 & 850 & 300 & 165 & 165 & 165 & 165 & 25 \\
\hline Enth MMBtu/h & -462.32 & -461.39 & -1.9533 & -1.9475 & -32.845 & -32.749 & -273.36 & -272.55 & -72.431 & -61.177 & 0.04276 & -16.089 & -16.095 & -17.02 & -12.449 & -18.232 \\
\hline Vapor mass fraction & 0 & 0 & 0 & 0 & 0 & 0 & 0 & 0 & 0 & 0.99 & 0 & 1 & 0.9977 & 0.51813 & 1 & \\
\hline $\begin{array}{l}\text { Total lb/h } \\
\text { Flowrates in lb/h }\end{array}$ & 211758 & 211758 & 287.032 & 287.032 & 4826.65 & 4826.65 & 40169.8 & 40169.8 & 10779.9 & 10779.9 & 236.741 & 7633.77 & 7633.77 & 7633.77 & 4021.9 & 30215.9 \\
\hline Methanol & 0 & 0 & 0 & 0 & 0 & 0 & 0 & 0 & 0 & 0 & 0 & 0 & 0 & 0 & 0 & 0 \\
\hline Water & 0 & 0 & 287.032 & 287.032 & 4826.65 & 4826.65 & 40169.8 & 40169.8 & 10779.9 & 10779.9 & 0 & 35.7899 & 35.7899 & 35.7899 & 24.6142 & 0 \\
\hline Carbon & 0 & 0 & 0 & 0 & 0 & 0 & 0 & 0 & 0 & 0 & 236.741 & 0 & 0 & 0 & 0 & 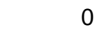 \\
\hline Hydrogen & 0 & 0 & 0 & 0 & 0 & 0 & 0 & 0 & 0 & 0 & 0 & 7.1799 & 7.1799 & 7.1799 & 7.3709 & 0 \\
\hline Carbon Dioxide & 0 & 0 & 0 & 0 & 0 & 0 & 0 & 0 & 0 & 0 & 0 & 3056.45 & 3056.45 & 3056.45 & 2717.17 & \\
\hline Methane & 0 & 0 & 0 & 0 & 0 & 0 & 0 & 0 & 0 & 0 & 0 & 555.132 & 555.132 & 555.132 & 547.207 & 0 \\
\hline Ethylene & 0 & 0 & 0 & 0 & 0 & 0 & 0 & 0 & 0 & 0 & 0 & 20.1408 & 20.1408 & 20.1408 & 17.3108 & \\
\hline Ethane & 0 & 0 & 0 & 0 & 0 & 0 & 0 & 0 & 0 & 0 & 0 & 59.0568 & 59.0568 & 59.0568 & 45.8336 & \\
\hline Propylene & 0 & 0 & 0 & 0 & 0 & 0 & 0 & 0 & 0 & 0 & 0 & 18.4619 & 18.4619 & 18.4619 & 9.3163 & \\
\hline Propane & 0 & 0 & 0 & 0 & 0 & 0 & 0 & 0 & 0 & 0 & 0 & 999.676 & 999.676 & 999.676 & 445.722 & \\
\hline I-Butane & 0 & 0 & 0 & 0 & 0 & 0 & 0 & 0 & 0 & 0 & 0 & 316.497 & 316.497 & 316.497 & 70.1319 & 28.6311 \\
\hline N-Butane & 0 & 0 & 0 & 0 & 0 & 0 & 0 & 0 & 0 & 0 & 0 & 181.221 & 181.221 & 181.221 & 28.0451 & 163.768 \\
\hline Cis-2-Butene & 0 & 0 & 0 & 0 & 0 & 0 & 0 & 0 & 0 & 0 & 0 & 148.699 & 148.699 & 148.699 & 19.847 & 169.297 \\
\hline I-Pentane & 0 & 0 & 0 & 0 & 0 & 0 & 0 & 0 & 0 & 0 & 0 & 769.807 & 769.807 & 769.807 & 46.3767 & 2985.15 \\
\hline N-Pentane & 0 & 0 & 0 & 0 & 0 & 0 & 0 & 0 & 0 & 0 & 0 & 189.163 & 189.163 & 189.163 & 8.3276 & 1033.24 \\
\hline Trans-2-Pentene & 0 & 0 & 0 & 0 & 0 & 0 & 0 & 0 & 0 & 0 & 0 & 202.562 & 202.562 & 202.562 & 9.3275 & 1091.47 \\
\hline Cyclopentane & 0 & 0 & 0 & 0 & 0 & 0 & 0 & 0 & 0 & 0 & 0 & 20.8642 & 20.8642 & 20.8642 & 0.5748 & 200.56 \\
\hline 2-2-DiMth-Butane & 0 & 0 & 0 & 0 & 0 & 0 & 0 & 0 & 0 & 0 & 0 & 187.616 & 187.616 & 187.616 & 5.0563 & 1755.77 \\
\hline 2-3-DiMth-Butane & 0 & 0 & 0 & 0 & 0 & 0 & 0 & 0 & 0 & 0 & 0 & 137.544 & 137.544 & 137.544 & 2.6484 & 1758.41 \\
\hline 2-Methylpentane & 0 & 0 & 0 & 0 & 0 & 0 & 0 & 0 & 0 & 0 & 0 & 129.333 & 129.333 & 129.333 & 2.2175 & 1758.83 \\
\hline 3-Methylpentane & 0 & 0 & 0 & 0 & 0 & 0 & 0 & 0 & 0 & 0 & 0 & 116.626 & 116.626 & 116.626 & 1.7854 & 1759.23 \\
\hline MthCyclopentane & 0 & 0 & 0 & 0 & 0 & 0 & 0 & 0 & 0 & 0 & 0 & 32.0084 & 32.0084 & 32.0084 & 0.3695 & 641.625 \\
\hline Benzene & 0 & 0 & 0 & 0 & 0 & 0 & 0 & 0 & 0 & 0 & 0 & 18.4904 & 18.4904 & 18.4904 & 0.2028 & 399.881 \\
\hline 2-4-DiMthPentane & 0 & 0 & 0 & 0 & 0 & 0 & 0 & 0 & 0 & 0 & 0 & 28.0406 & 28.0406 & 28.0406 & 0.2046 & 754.446 \\
\hline Cyclohexane & 0 & 0 & 0 & 0 & 0 & 0 & 0 & 0 & 0 & 0 & 0 & 15.3705 & 15.3705 & 15.3705 & 0.1219 & 411.938 \\
\hline 2-Methylhexane & 0 & 0 & 0 & 0 & 0 & 0 & 0 & 0 & 0 & 0 & 0 & 20.6567 & 20.6567 & 20.6567 & 0.0972 & 754.54 \\
\hline 3-Methylhexane & 0 & 0 & 0 & 0 & 0 & 0 & 0 & 0 & 0 & 0 & 0 & 19.5156 & 19.5156 & 19.5156 & 0.0855 & 754.549 \\
\hline 2-2-4-TriMth-C5 & 0 & 0 & 0 & 0 & 0 & 0 & 0 & 0 & 0 & 0 & 0 & 15.4364 & 15.4364 & 15.4364 & 0.0519 & 754.579 \\
\hline MthCyclohexane & 0 & 0 & 0 & 0 & 0 & 0 & 0 & 0 & 0 & 0 & 0 & 12.9635 & 12.9635 & 12.9635 & 0.0466 & 641.924 \\
\hline 33-2Mth-1-Butene & 0 & 0 & 0 & 0 & 0 & 0 & 0 & 0 & 0 & 0 & 0 & 115.995 & 115.995 & 115.995 & 4.1292 & 804.056 \\
\hline Toluene & 0 & 0 & 0 & 0 & 0 & 0 & 0 & 0 & 0 & 0 & 0 & 44.1962 & 44.1962 & 44.1962 & 0.1362 & 2498.04 \\
\hline 2-3-3-TriMth-C5 & 0 & 0 & 0 & 0 & 0 & 0 & 0 & 0 & 0 & 0 & 0 & 6.4637 & 6.4637 & 6.4637 & 0.0116 & 503.11 \\
\hline 2-3-DiMth-Hexane & 0 & 0 & 0 & 0 & 0 & 0 & 0 & 0 & 0 & 0 & 0 & 6.0861 & 6.0861 & 6.0861 & 0.0091 & 503.113 \\
\hline 3-Methylheptane & 0 & 0 & 0 & 0 & 0 & 0 & 0 & 0 & 0 & 0 & 0 & 5.4256 & 5.4256 & 5.4256 & 0.0065 & 504.032 \\
\hline 23-2Mth-1-Butene & 0 & 0 & 0 & 0 & . & 0 & 0 & 0 & 0 & 0 & 0 & 69.2526 & 69.2526 & 69.2526 & 1.4445 & 813.116 \\
\hline P-Xylene & 0 & 0 & 0 & 0 & 0 & 0 & 0 & 0 & 0 & 0 & 0 & 7.4536 & 7.4536 & 7.4536 & 0.0061 & 1037.65 \\
\hline M-Xylene & 0 & 0 & 0 & 0 & 0 & 0 & 0 & 0 & 0 & 0 & 0 & 15.9148 & 15.9148 & 15.9148 & 0.0123 & 2286.72 \\
\hline O-Xylene & 0 & 0 & 0 & 0 & 0 & 0 & 0 & 0 & 0 & 0 & 0 & 5.5591 & 5.5591 & 5.5591 & 0.0034 & 940.056 \\
\hline M-Ethyltoluene & 0 & 0 & 0 & 0 & 0 & 0 & 0 & 0 & 0 & 0 & 0 & 1.6837 & 1.6837 & 1.6837 & 0.0004 & 487.941 \\
\hline 23-2Mth-2-Butene & 0 & 0 & 0 & 0 & 0 & 0 & 0 & 0 & 0 & 0 & 0 & 31.861 & 31.861 & 31.861 & 0.3667 & 632.078 \\
\hline Dimethyl Ether & 0 & 0 & 0 & 0 & 0 & 0 & 0 & 0 & 0 & 0 & 0 & 0 & 0 & 0 & 0 & \\
\hline 1-2-4-Trimethylb & 0 & 0 & 0 & 0 & 0 & 0 & 0 & 0 & 0 & 0 & 0 & 3.3439 & 3.3439 & 3.3439 & 0.0005 & 1366.17 \\
\hline 1-2-4-5-TetMthBz & 0 & 0 & 0 & 0 & 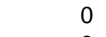 & 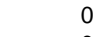 & 0 & 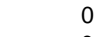 & 0 & 0 & 0 & 0.3652 & 0.3652 & 0.3652 & 0 & 21.9705 \\
\hline Naphthalene & 0 & 0 & 0 & 0 & 0 & 0 & 0 & 0 & 0 & 0 & 0 & 0.223 & 0.223 & 0.223 & 0 & 0.0005 \\
\hline 2-MthNaphthalene & 0 & 0 & 0 & 0 & 0 & 0 & 0 & 0 & 0 & 0 & 0 & 0.012 & 0.012 & 0.012 & 0 & 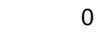 \\
\hline Oxygen & 0 & 0 & 0 & 0 & 0 & 0 & 0 & 0 & 0 & 0 & 0 & 0 & 0 & 0 & 0 & 0 \\
\hline Nitrogen & 0 & 0 & 0 & 0 & 0 & 0 & 0 & 0 & 0 & 0 & 0 & 5.6387 & 5.6387 & 5.6387 & 5.7097 & \\
\hline 1-2-3-4-Tetramet & 0 & 0 & 0 & 0 & 0 & 0 & 0 & 0 & 0 & 0 & 0 & 0 & 0 & 0 & 0 & \\
\hline 1-1-Dichloro-2-2 & 211758 & 211758 & 0 & 0 & 0 & 0 & 0 & 0 & 0 & 0 & 0 & 0 & 0 & 0 & 0 & \\
\hline
\end{tabular}


Appendix B. Heat and Material Balances for the Directlyheated Gasifier Case 

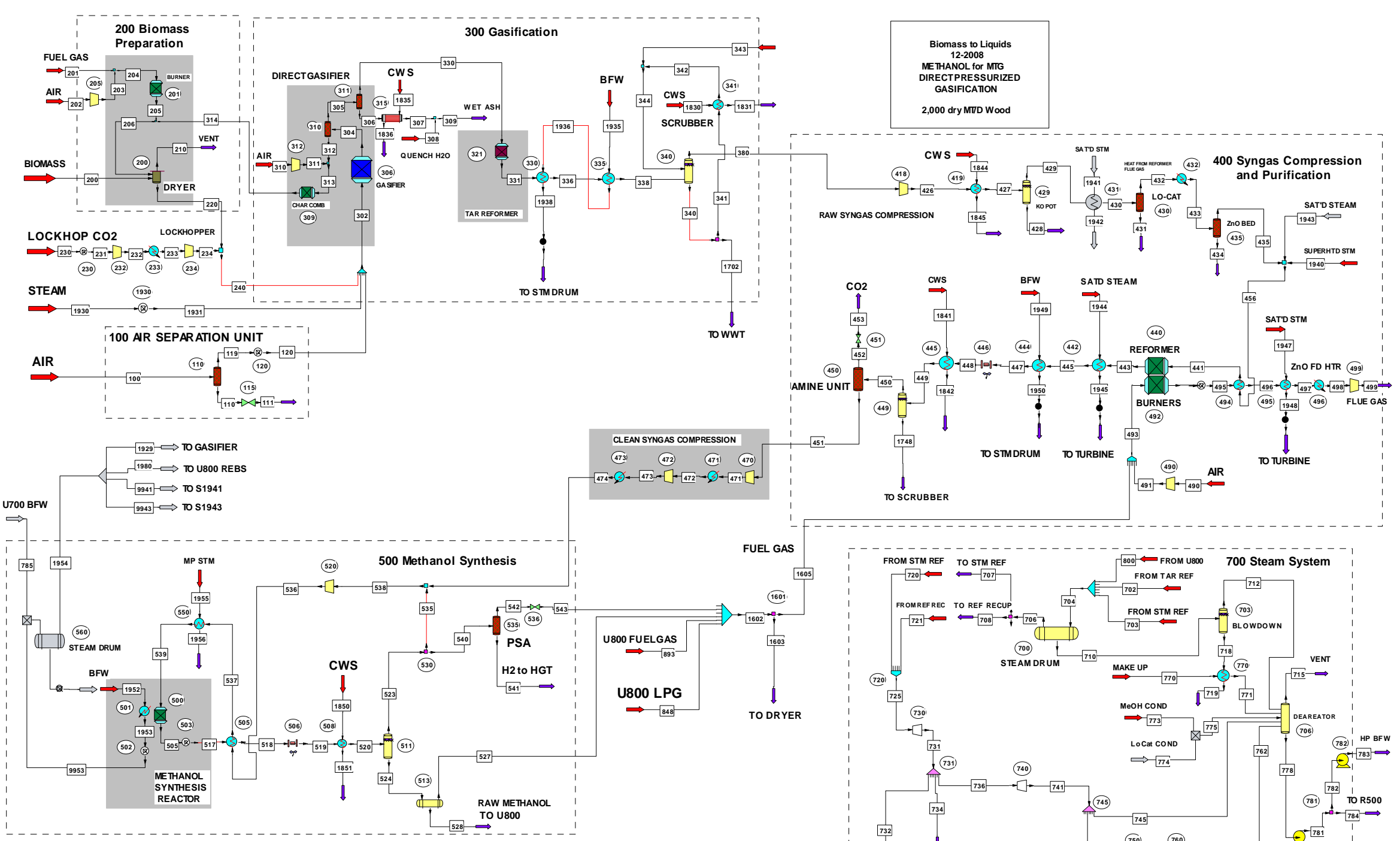
Table B-1 Steam Results for the Directly-heated Gasifier Case - Feed Prep through Methanol Synthesis 


\begin{tabular}{|c|c|c|c|c|c|c|c|c|c|c|c|c|c|c|c|c|c|c|c|c|c|c|c|c|}
\hline Stream No. & 100 & 110 & 111 & 119 & 120 & 200 & 201 & 202 & 203 & 204 & 205 & 206 & 210 & 220 & 230 & 231 & 232 & 233 & 234 & 240 & 246 & 302 & 304 & 305 \\
\hline Temp F & 60 & 60 & 43.232 & 60 & 60 & 60 & 58.9967 & 60 & 76.9876 & 73.5884 & 3131.99 & 3518.63 & 241.951 & 240 & 70 & 70 & 329.524 & 140 & 360.069 & 239.616 & 287 & 357.687 & 1600 & 1600 \\
\hline Pres psia & 14.696 & 350 & 25 & 350 & 350 & 25 & & 14.696 & & & & & & 16 & 22 & 22 & 100 & 100 & 330 & 330 & 35 & 330 & 330 & 330 \\
\hline Enth MMBtu/h & -26.112 & -28.339 & -28.339 & -0.27723 & -0.27723 & -1744.9 & -12.57 & -0.26333 & -0.00461 & -12.575 & -12.576 & -2.9397 & -1104.3 & -643.52 & -23.166 & -23.166 & -22.829 & -23.089 & -22.801 & -666.32 & -2340.3 & -903.19 & -857.61 & $\begin{array}{l}-865.95 \\
0.98293\end{array}$ \\
\hline Vapor mass fraction & 0.99099 & 0.97525 & 0.9781 & & & 0 & & & & & & & 1 & & & & 1 & & & 0.19591 & & 0.64635 & 0.98301 & $\begin{array}{r}0.98293 \\
225388\end{array}$ \\
\hline $\begin{array}{l}\text { Total l lb/h } \\
\text { Flowrates in Ib/h }\end{array}$ & 203421 & 157071 & 157071 & 46350.4 & 46350.4 & 367437 & 6413.24 & 63000 & 63000 & 69413.3 & 69413.3 & 192327 & 350993 & 208771 & 6023.08 & 6023.08 & 6023.08 & 6023.08 & 6023.08 & 214794 & 411318 & 302899 & 302899 & 295388 \\
\hline Oxygen & 46153.1 & 39.6902 & 39.6902 & 46113.4 & 46113.4 & 0 & 0 & 14673.7 & 14673.7 & 14673.7 & 3728.45 & 7271.14 & 7271.14 & 0 & 0 & 0 & 0 & 0 & 0 & 0 & 0 & 46113.4 & 307.108 & \\
\hline Nitrogen & 150630 & 150547 & 150547 & 82.8463 & 82.8463 & 0 & 107.807 & 48326.4 & 48326.4 & 48434.2 & 48310.2 & 136506 & 136506 & 0 & 0 & 0 & 0 & 0 & 0 & 0 & 0 & 82.8463 & 120.38 & 82.8463 \\
\hline Argon & 2568.97 & 2414.83 & 2414.83 & 154.138 & 154.138 & 0 & 19.5242 & & & 19.5242 & 19.5242 & 19.5242 & 19.5242 & 0 & 0 & 0 & 0 & 0 & 0 & 0 & 0 & 154.138 & 154.138 & 154.138 \\
\hline Carbon & & & & & & 0 & & 0 & 0 & & & 0.0002 & 0.0002 & 0 & 0 & 0 & 0 & 0 & 0 & 0 & 0 & & 6254.95 & \\
\hline Hydrogen & 0 & 0 & 0 & 0 & 0 & 0 & 438.492 & 0 & 0 & 438.492 & 0.9094 & 11.4084 & 11.4084 & 0 & 0 & 0 & 0 & 0 & 0 & 0 & 0 & 0 & 4562.56 & 3655.06 \\
\hline Carbon Monoxide & 0 & 0 & 0 & 0 & 0 & 0 & 2741.24 & 0 & 0 & 2741.24 & 37.9279 & 1021.48 & 1021.48 & 0 & 0 & 0 & 0 & 0 & 0 & 0 & 0 & 0 & 49736.8 & 49736.8 \\
\hline Carbon Dioxide & 100.293 & 100.293 & 100.293 & 0 & 0 & 0 & 1462.82 & 0 & 0 & 1462.82 & 10338.6 & 31712.5 & 31712.5 & 0 & 6023.08 & 6023.08 & 6023.08 & 6023.08 & 6023.08 & 6023.08 & 0 & 6023.08 & 135752 & 135752 \\
\hline Methane & & & & 0 & 0 & 0 & 465.165 & 0 & 0 & 465.165 & & & & 0 & & 0 & 0 & 0 & 0 & 0 & 0 & 0 & 21336.9 & 21336.9 \\
\hline Acetylene & 0 & 0 & 0 & 0 & 0 & 0 & & 0 & 0 & & 0 & 0 & 0 & 0 & 0 & 0 & 0 & 0 & 0 & 0 & 0 & 0 & & \\
\hline Ethylene & 0 & 0 & 0 & 0 & 0 & 0 & 1.7048 & 0 & 0 & 1.7048 & 0.0001 & 0.0001 & 0.0001 & 0 & 0 & 0 & 0 & 0 & 0 & 0 & 0 & 0 & 1041.31 & 1041.31 \\
\hline Ethane & 0 & 0 & 0 & 0 & 0 & 0 & 4.3219 & 0 & 0 & 4.3219 & 0.0001 & 0.0001 & 0.0001 & 0 & 0 & 0 & 0 & 0 & 0 & 0 & 0 & 0 & 1948.76 & 1948.76 \\
\hline Propane & 0 & 0 & 0 & 0 & 0 & 0 & 224.917 & 0 & 0 & 224.917 & 0.0001 & 0.0001 & 0.0001 & 0 & 0 & 0 & 0 & 0 & 0 & 0 & 0 & 0 & & \\
\hline Water & 3968.93 & 3968.93 & 3968.93 & 0 & 0 & 183719 & 2.6508 & 0 & 0 & 2.6508 & 6712.12 & 14728.7 & 173395 & 25052.5 & 0 & 0 & 0 & 0 & 0 & 25052.5 & 411318 & 66806.3 & 62887.6 & 62887.6 \\
\hline Sulphur & & & 0 & 0 & 0 & & & 0 & 0 & & & 0.0002 & 0.0002 & 0 & 0 & 0 & 0 & 0 & 0 & 0 & 0 & 0 & 3.0449 & \\
\hline Carbonyl Sulfide & 0 & 0 & 0 & 0 & 0 & 0 & 0 & 0 & 0 & 0 & 0 & 0 & 0 & 0 & 0 & 0 & 0 & 0 & 0 & 0 & 0 & 0 & 0 & \\
\hline Hydrogen Sulfide & 0 & 0 & 0 & 0 & 0 & 0 & 0 & 0 & 0 & 0 & 0 & 0 & 0 & 0 & 0 & 0 & 0 & 0 & 0 & 0 & 0 & 0 & 35.064 & 35.064 \\
\hline Ammonia & 0 & 0 & 0 & 0 & 0 & 0 & 0 & 0 & 0 & 0 & 0 & 0 & 0 & 0 & 0 & 0 & 0 & 0 & 0 & 0 & 0 & 0 & 1312.59 & 1312.59 \\
\hline HydrogenChloride & 0 & 0 & 0 & 0 & 0 & 0 & 0 & 0 & 0 & 0 & 0 & 0 & 0 & 0 & 0 & 0 & 0 & 0 & 0 & 0 & 0 & 0 & 18.5296 & 18.5296 \\
\hline Silicon Dioxide & 0 & 0 & 0 & 0 & 0 & 0 & 0 & 0 & 0 & 0 & 0 & 0 & 0 & 0 & 0 & 0 & 0 & 0 & 0 & 0 & 0 & 0 & & \\
\hline Calcium Oxide & 0 & 0 & 0 & 0 & 0 & 0 & 0 & 0 & 0 & 0 & 0 & 0 & 0 & 0 & 0 & 0 & 0 & 0 & 0 & 0 & 0 & 0 & 4960.38 & 4960.38 \\
\hline Benzene & 0 & 0 & 0 & 0 & 0 & 0 & 0.0172 & 0 & 0 & 0.0172 & 0.0002 & 0.0002 & 0.0002 & 0 & 0 & 0 & 0 & 0 & 0 & 0 & 0 & 0 & 8488.38 & 8488.38 \\
\hline Naphthalene & 0 & 0 & 0 & 0 & 0 & 0 & 0.0182 & 0 & 0 & 0.0182 & 0.0002 & 0.0002 & 0.0002 & 0 & 0 & 0 & 0 & 0 & 0 & 0 & 0 & 0 & 3977.61 & 3977.61 \\
\hline Hybrid Poplar Ch & 0 & 0 & 0 & 0 & 0 & 183719 & 0 & 0 & 0 & & 0 & & & 183719 & 0 & 0 & 0 & 0 & 0 & 183719 & 0 & 183719 & 0 & \\
\hline Sulfur Dioxide & 0 & 0 & 0 & 0 & 0 & 0 & 0 & 0 & 0 & 0 & 0 & 6.0849 & 6.0849 & 0 & 0 & 0 & 0 & 0 & 0 & 0 & 0 & 0 & 0 & 0 \\
\hline Hydrogen Cyanide & 0 & 0 & 0 & 0 & 0 & 0 & 0 & 0 & 0 & 0 & 0 & & & 0 & 0 & 0 & 0 & 0 & 0 & 0 & 0 & 0 & 0 & 0 \\
\hline Nitric Oxide & 0 & 0 & 0 & 0 & 0 & 0 & 0 & 0 & 0 & & 265.563 & 1050.51 & 1050.51 & 0 & 0 & 0 & 0 & 0 & 0 & 0 & 0 & 0 & 0 & 0 \\
\hline Methanol & 0 & 0 & 0 & 0 & 0 & 0 & 129.046 & 0 & 0 & 129.046 & 0.0001 & 0.0001 & 0.0001 & 0 & 0 & 0 & 0 & 0 & 0 & 0 & 0 & 0 & 0 & 0 \\
\hline Ethanol & 0 & 0 & 0 & 0 & 0 & 0 & & 0 & 0 & & & & & 0 & 0 & 0 & 0 & 0 & 0 & 0 & 0 & 0 & 0 & 0 \\
\hline Propylene & 0 & 0 & 0 & 0 & 0 & 0 & 2.1402 & 0 & 0 & 2.1402 & 0.0001 & 0.0001 & 0.0001 & 0 & 0 & 0 & 0 & 0 & 0 & 0 & 0 & 0 & 0 & 0 \\
\hline I-Butane & 0 & 0 & 0 & 0 & 0 & 0 & 446.04 & 0 & 0 & 446.04 & 0.0001 & 0.0001 & 0.0001 & 0 & 0 & 0 & 0 & 0 & 0 & 0 & 0 & 0 & 0 & \\
\hline N-Butane & 0 & 0 & 0 & 0 & 0 & 0 & 133.11 & 0 & 0 & 133.11 & 0.0001 & 0.0001 & 0.0001 & 0 & 0 & 0 & 0 & 0 & 0 & 0 & 0 & 0 & 0 & \\
\hline Cis-2-Butene & 0 & 0 & 0 & 0 & 0 & 0 & 74.3971 & 0 & 0 & 74.3971 & 0.0001 & 0.0001 & 0.0001 & 0 & 0 & 0 & 0 & 0 & 0 & 0 & 0 & 0 & 0 & \\
\hline I-Pentane & 0 & 0 & 0 & 0 & 0 & 0 & 132.08 & 0 & 0 & 132.08 & 0.0001 & 0.0001 & 0.0001 & 0 & 0 & 0 & 0 & 0 & 0 & 0 & 0 & 0 & 0 & \\
\hline N-Pentar & 0 & 0 & 0 & 0 & 0 & 0 & 9.1253 & 0 & 0 & 9.1253 & 0.0001 & 0.0001 & 0.0001 & 0 & 0 & 0 & 0 & 0 & 0 & 0 & 0 & 0 & 0 & \\
\hline Trans-2 & 0 & 0 & 0 & 0 & 0 & 0 & 15.7974 & 0 & 0 & 15.7974 & 0.0001 & 0.0001 & 0.0001 & 0 & 0 & 0 & 0 & 0 & 0 & 0 & 0 & 0 & 0 & \\
\hline Cyclopenta & 0 & 0 & 0 & 0 & 0 & 0 & 0.0671 & 0 & 0 & 0.0671 & 0.0001 & 0.0001 & 0.0001 & 0 & 0 & 0 & 0 & 0 & 0 & 0 & 0 & 0 & 0 & \\
\hline 2-2-DiM & 0 & 0 & 0 & 0 & 0 & 0 & 0.4728 & 0 & 0 & 0.4728 & 0.0001 & 0.0001 & 0.0001 & 0 & 0 & 0 & 0 & 0 & 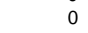 & 0 & 0 & 0 & 0 & \\
\hline 2-3-DiN & 0 & 0 & 0 & 0 & 0 & 0 & 0.226 & 0 & 0 & 0.226 & 0.0001 & 0.0001 & 0.0001 & 0 & 0 & 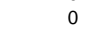 & 0 & 0 & 0 & 0 & 0 & 0 & 0 & \\
\hline 2-Metl & 0 & 0 & 0 & 0 & 0 & 0 & 0.3121 & 0 & 0 & 0.3121 & 0.0001 & 0.0001 & 0.0001 & 0 & 0 & 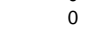 & 0 & 0 & 0 & 0 & 0 & 0 & 0 & \\
\hline 3-Met & 0 & 0 & 0 & 0 & 0 & 0 & 0.1516 & 0 & 0 & 0.1516 & 0.0001 & 0.0001 & 0.0001 & 0 & 0 & 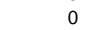 & 0 & 0 & 0 & 0 & 0 & 0 & 0 & \\
\hline MthC & 0 & 0 & 0 & 0 & 0 & 0 & 0.0314 & 0 & 0 & 0.0314 & 0.0001 & 0.0001 & 0.0001 & 0 & 0 & 0 & 0 & 0 & 0 & 0 & 0 & 0 & 0 & \\
\hline$-4-D$ & 0 & 0 & 0 & 0 & 0 & 0 & 0.01 & 0 & 0 & 0.0173 & 0.0002 & & & 0 & 0 & 0 & 0 & 0 & 0 & 0 & 0 & 0 & 0 & \\
\hline Cyclo & 0 & 0 & 0 & 0 & 0 & 0 & 0.01 & 0 & 0 & 0.0104 & 0.0001 & 0. & 01 & 0 & 0 & 0 & 0 & 0 & 0 & 0 & 0 & 0 & 0 & 0 \\
\hline 2-Me & 0 & 0 & 0 & 0 & 0 & 0 & 0.0 & 0 & 0 & 0.0082 & 0.0002 & & 0 & 0 & 0 & 0 & 0 & . & 0 & 0 & 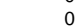 & 0 & 0 & \\
\hline 3-Met & 0 & 0 & 0 & 0 & 0 & 0 & 0.00 & 0 & 0 & 0.0072 & 0.0002 & 0. & 0 & 0 & 0 & 0 & 0 & 0 & 0 & 0 & 0 & 0 & 0 & 0 \\
\hline & 0 & 0 & 0 & 0 & 0 & 0 & 0.00 & 0 & 0 & 0.0044 & 0.0002 & & & 0 & 0 & 0 & 0 & 0 & 0 & 0 & 0 & & 0 & \\
\hline MthC & 0 & 0 & 0 & 0 & 0 & 0 & 0.004 & 0 & 0 & 0.004 & 0.0002 & 0. & 2 & 0 & 0 & 0 & 0 & 0 & 0 & 0 & 0 & 0 & 0 & 0 \\
\hline $33-2$ & 0 & 0 & 0 & 0 & 0 & 0 & 1.1781 & 0 & 0 & $\begin{array}{l}1.004 \\
1.1781\end{array}$ & 0.0001 & 0.0001 & 0.0001 & 0 & 0 & 0 & 0 & 0 & 0 & 0 & 0 & 0 & 0 & 0 \\
\hline Dimethyl Ether & 0 & 0 & 0 & 0 & 0 & 0 & 0 & 0 & 0 & 0 & & & 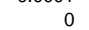 & 0 & 0 & 0 & 0 & 0 & 0 & 0 & 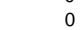 & 0 & 0 & 0 \\
\hline Toluen & 0 & 0 & 0 & 0 & 0 & 0 & 0.0116 & 0 & 0 & 0.0116 & 0.0002 & 0.0002 & 0.0002 & 0 & 0 & 0 & 0 & 0 & 0 & 0 & 0 & 0 & 0 & 0 \\
\hline & 0 & 0 & 0 & 0 & 0 & 0 & 0.0003 & 0 & 0 & 0.0003 & 0.0002 & 0.0002 & 0.0002 & 0 & 0 & 0 & 0 & 0 & 0 & 0 & 0 & 0 & 0 & 0 \\
\hline M-Xyle & 0 & 0 & 0 & 0 & 0 & 0 & 0.001 & 0 & 0 & 0.001 & 0.0002 & 0.0002 & 0.0002 & 0 & 0 & 0 & 0 & 0 & 0 & 0 & 0 & 0 & 0 & 0 \\
\hline$P-x y l$ & 0 & 0 & 0 & 0 & 0 & 0 & 0.0005 & 0 & 0 & 0.0005 & 0.0002 & 0.0002 & 0.0002 & 0 & 0 & 0 & 0 & 0 & 0 & 0 & 0 & 0 & 0 & 0 \\
\hline M-Etl & 0 & 0 & 0 & 0 & 0 & 0 & & 0 & 0 & & 0 & & 0 & 0 & 0 & 0 & 0 & 0 & 0 & 0 & 0 & 0 & 0 & 0 \\
\hline $1-2-4$ & 0 & 0 & 0 & 0 & 0 & 0 & & 0 & 0 & & & & & 0 & 0 & 0 & 0 & 0 & 0 & 0 & 0 & 0 & 0 & 0 \\
\hline & 0 & 0 & 0 & 0 & 0 & 0 & 0.0009 & 0 & 0 & 0.0009 & 0.0002 & 0.0002 & 0.0002 & 0 & 0 & 0 & 0 & 0 & 0 & 0 & 0 & 0 & 0 & 0 \\
\hline $1-2-2$ & 0 & 0 & 0 & 0 & 0 & 0 & & 0 & 0 & & & & & 0 & 0 & 0 & 0 & 0 & 0 & 0 & 0 & 0 & 0 & 0 \\
\hline $23-2$ & 0 & 0 & 0 & 0 & 0 & 0 & 0.125 & 0 & 0 & 0.125 & 0.0002 & 0.0 & 0.0002 & 0 & 0 & 0 & 0 & 0 & 0 & 0 & 0 & 0 & 0 & 0 \\
\hline & 0 & . & 0 & 0 & 0 & 0 & 0.0 & 0 & 0 & 0.0 & & & 0.0001 & 0 & 0 & 0 & 0 & 0 & 0 & 0 & 0 & 0 & 0 & \\
\hline & 0 & 0 & 0 & 0 & 0 & 0 & & 0 & 0 & & & & & 0 & 0 & 0 & 0 & 0 & 0 & 0 & 0 & 0 & 0 & \\
\hline $2-3-D$ & 0 & 0 & 0 & 0 & 0 & 0 & 0.0 & 0 & 0 & 0.0 & & 2 & 0.0002 & 0 & 0 & 0 & 0 & 0 & 0 & 0 & 0 & 0 & 0 & \\
\hline 2-3-3-TriMth-C5 & 0 & 0 & 0 & 0 & 0 & 0 & 0.001 & 0 & 0 & 0.001 & 0.0002 & 0.0002 & 0.0002 & 0 & 0 & 0 & 0 & 0 & 0 & 0 & 0 & 0 & 0 & \\
\hline 1-2-3-4-Tetramet & 0 & 0 & 0 & 0 & 0 & 0 & 0.1585 & 0 & 0 & 0.1585 & 0.0001 & 0.0001 & 0.0001 & 0 & 0 & 0 & 0 & 0 & 0 & 0 & 0 & 0 & 0 & \\
\hline
\end{tabular}




\begin{tabular}{|c|c|c|c|c|c|c|c|c|c|c|c|c|c|c|c|c|c|c|c|c|c|c|c|c|}
\hline Stream No. & 431 & 432 & 433 & 434 & 435 & 441 & 443 & 445 & 447 & 448 & 449 & 450 & 451 & 452 & 453 & 456 & 471 & 472 & 473 & 474 & 490 & 491 & 493 & 495 \\
\hline Temp F & 120 & 120 & 707 & 707 & 707 & 950 & 1652 & 1389.21 & 300.001 & 150 & 110 & 110 & 120 & 120 & 60.0217 & 655.031 & 203.335 & 150 & 249.847 & 150 & 60 & 124.488 & 111.107 & 1800 \\
\hline Pres psia & 445 & 445 & 445 & 440 & 440 & 437 & 422 & 421 & 421 & 421 & 418.5 & 418.5 & 413.5 & 413.5 & & & 600 & 600 & 915 & 915 & 14.696 & & 20 & 20 \\
\hline Enth MMBtu/h & 0 & -697.36 & -639.53 & -0.06147 & -639.47 & -1683.5 & -1436.9 & -1498 & -1837.5 & -1928.7 & -1942 & -775.24 & -232.64 & -542.85 & -542.85 & -1744.8 & -225.61 & -230.12 & -221.59 & -230.11 & -1.507 & 4.1197 & -81.03 & -328.82 \\
\hline Vapor mass fraction & 0 & 0.99918 & 1 & & 1 & 1 & 1 & & 0.73847 & 0.62026 & 0.61558 & & & 0.99549 & 0.99792 & & 1 & 1 & & & & & & \\
\hline \multirow{2}{*}{\multicolumn{25}{|c|}{ 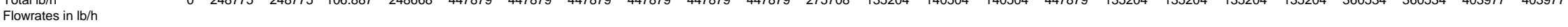 }} \\
\hline & & & & & & & & & & & & & & & & & & & & & & & & \\
\hline xygen & 0 & & & 0 & & 0 & & & 0 & 0 & & 0 & 0 & 0 & 0 & 0 & 0 & 0 & 0 & 0 & 83974 & 83974 & 83974 & 10570.9 \\
\hline Nitrogen & 0 & 838.459 & 838.459 & 0 & 838.459 & 838.459 & 838.459 & 838.459 & 838.459 & 838.459 & 838.459 & 838.459 & 838.459 & 0 & 0 & 838.459 & 838.459 & 838.459 & 838.459 & 838.459 & 276560 & 276560 & 277291 & 277270 \\
\hline Argon & 0 & 154.109 & 154.109 & 0 & 154.109 & 154.109 & 154.109 & 154.109 & 154.109 & 154.109 & 154.109 & 154.103 & 154.103 & 0 & 0 & 154.109 & 154.103 & 154.103 & 154.103 & 154.103 & & & 132.257 & 132.257 \\
\hline Carbon & 0 & & & 0 & & & & & & & & & & 0 & 0 & & & & & & 0 & 0 & & \\
\hline Hydrogen & 0 & 8375.24 & 8375.24 & 0 & 8375.24 & 8375.24 & 15275.8 & 15275.8 & 15275.8 & 15275.8 & 15275.8 & 15275.7 & 15275.7 & 0 & 0 & 8375.24 & 15275.7 & 15275.7 & 15275.7 & 15275.7 & 0 & 0 & 2970.43 & 0.0011 \\
\hline Carbon Monoxide & 0 & 82524.4 & 82524.4 & 0 & 82524.4 & 82524.4 & 100041 & 100041 & 100041 & 100041 & 100041 & 100041 & 100041 & 0 & 0 & 82524.4 & 100041 & 100041 & 100041 & 100041 & 0 & 0 & 18568.4 & 0.0157 \\
\hline Carbon Dioxide & 0 & 135338 & 135338 & 0 & 135338 & 135338 & 156029 & 156029 & 156029 & 156029 & 156029 & 155051 & 15505.1 & 139546 & 139546 & 135338 & 15505.1 & 15505.1 & 15505.1 & 15505.1 & 0 & 0 & 9908.94 & 70436 \\
\hline Methane & & 17066.5 & 17066.5 & 0 & 17066.5 & 17066.5 & 3389.91 & 3389.91 & 3389.91 & 3389.91 & 3389.91 & 3389.86 & 3389.86 & & & 17066.5 & 3389.86 & 3389.86 & 3389.86 & 3389.86 & 0 & 0 & 3151.01 & 0.0002 \\
\hline Acetylene & 0 & & & 0 & & & & & & & & & & 0 & 0 & & & & & & 0 & 0 & & \\
\hline Ethylene & & 519.453 & 519.453 & 0 & 519.453 & 519.453 & 0.0456 & 0.0456 & 0.0456 & 0.0456 & 0.0456 & 0.0456 & 0.0456 & 0 & 0 & 519.453 & 0.0456 & 0.0456 & 0.0456 & 0.0456 & 0 & 0 & 11.5483 & 0.0004 \\
\hline Ethane & 0 & 194.488 & 194.488 & 0 & 194.488 & 194.488 & 0.0997 & 0.0997 & 0.0997 & 0.0997 & 0.0997 & 0.0997 & 0.0997 & 0 & 0 & 194.488 & 0.0997 & 0.0997 & 0.0997 & 0.0997 & 0 & 0 & 29.2772 & 0.0004 \\
\hline Propane & 0 & & & 0 & & & & & & & & & 0 & 0 & 0 & & 0 & 0 & 0 & 0 & 0 & 0 & 1523.62 & 0.0006 \\
\hline Water & 0 & 1145.16 & 1145.16 & 0 & 1145.16 & 200356 & 172152 & 172152 & 172152 & 172152 & 172152 & 957.713 & 0 & 957.713 & 957.713 & 200356 & 0 & 0 & 0 & 0 & 0 & 0 & 17.9567 & 45524 \\
\hline Sulphur & 0 & & 0 & 0 & & & & & & & 0 & 0 & 0 & 0 & 0 & 0 & 0 & 0 & 0 & 0 & 0 & 0 & 0 & \\
\hline Carbonyl Sulfide & 0 & & 0 & 0 & 0 & 0 & 0 & 0 & 0 & 0 & 0 & 0 & 0 & 0 & 0 & 0 & 0 & 0 & 0 & 0 & 0 & 0 & 0 & \\
\hline Hydrogen Sulfide & 0 & 34.8084 & 34.8084 & 34.8084 & 0 & 0 & 0 & 0 & 0 & 0 & 0 & 0 & 0 & 0 & 0 & 0 & 0 & 0 & 0 & 0 & 0 & 0 & 0 & \\
\hline Ammonia & 0 & 72.0787 & 72.0787 & 72.0787 & 0 & 0 & 0 & 0 & 0 & 0 & 0 & 0 & 0 & 0 & 0 & 0 & 0 & 0 & 0 & 0 & 0 & 0 & 0 & \\
\hline HydrogenChloride & 0 & & & 0 & 0 & 0 & 0 & 0 & 0 & 0 & 0 & 0 & 0 & 0 & 0 & 0 & 0 & 0 & 0 & 0 & 0 & 0 & 0 & \\
\hline Silicon Dioxide & 0 & 0 & 0 & 0 & 0 & 0 & 0 & 0 & 0 & 0 & 0 & 0 & 0 & 0 & 0 & 0 & 0 & 0 & 0 & 0 & 0 & 0 & 0 & \\
\hline Calcium Oxide & 0 & & 0 & 0 & 0 & 0 & 0 & 0 & 0 & 0 & 0 & 0 & 0 & 0 & 0 & 0 & 0 & 0 & 0 & 0 & 0 & 0 & 0 & \\
\hline Benzene & 0 & 2467.8 & 2467.8 & 0 & 2467.8 & 2467.8 & 0 & 0 & 0 & 0 & 0 & 0 & 0 & 0 & 0 & 2467.8 & 0 & 0 & 0 & 0 & 0 & 0 & 0.1168 & 0.0009 \\
\hline Naphthalene & 0 & 44.1134 & 44.1134 & 0 & 44.1134 & 44.1134 & 0 & 0 & 0 & 0 & 0 & 0 & 0 & 0 & 0 & 44.1134 & 0 & 0 & 0 & 0 & 0 & 0 & 0.1234 & 0.0012 \\
\hline Hybrid Poplar Ch & 0 & & 0 & 0 & 0 & 0 & 0 & 0 & 0 & 0 & 0 & 0 & 0 & 0 & 0 & 0 & 0 & 0 & 0 & 0 & 0 & 0 & & \\
\hline Sulfur Dioxide & 0 & 0 & 0 & 0 & 0 & 0 & 0 & 0 & 0 & 0 & 0 & 0 & 0 & 0 & 0 & 0 & 0 & 0 & 0 & 0 & 0 & 0 & 0 & \\
\hline Hydrogen Cyanide & 0 & 0 & 0 & 0 & 0 & 0 & 0 & 0 & 0 & 0 & 0 & 0 & 0 & 0 & 0 & 0 & 0 & 0 & 0 & 0 & 0 & 0 & 0 & \\
\hline Nitric Oxide & 0 & 0 & 0 & 0 & 0 & 0 & 0 & 0 & 0 & 0 & 0 & 0 & 0 & 0 & 0 & 0 & 0 & 0 & 0 & 0 & 0 & 0 & 0 & 43.0564 \\
\hline Methanol & 0 & 0 & 0 & 0 & 0 & 0 & 0.047 & 0.047 & 0.047 & 0.047 & 0.047 & 0.0026 & 0.0026 & 0 & 0 & 0 & 0.0026 & 0.0026 & 0.0026 & 0.0026 & 0 & 0 & 874.154 & 0.0005 \\
\hline Ethanol & 0 & 0 & 0 & 0 & 0 & 0 & & & 0 & 0 & 0 & & 0 & 0 & 0 & 0 & & 0 & & & 0 & 0 & & \\
\hline Propylene & 0 & 0 & 0 & 0 & 0 & 0 & 0 & 0 & 0 & 0 & 0 & 0 & 0 & 0 & 0 & 0 & 0 & 0 & 0 & 0 & 0 & 0 & 14.4982 & 0.0006 \\
\hline I-Butane & 0 & 0 & 0 & 0 & 0 & 0 & 0 & 0 & 0 & 0 & 0 & 0 & 0 & 0 & 0 & 0 & 0 & 0 & 0 & 0 & 0 & 0 & 3021.54 & 0.0007 \\
\hline N-Butane & 0 & 0 & 0 & 0 & 0 & 0 & 0 & 0 & 0 & 0 & 0 & 0 & 0 & 0 & 0 & 0 & 0 & 0 & 0 & 0 & 0 & 0 & 901.71 & 0.0007 \\
\hline Cis-2-Butene & 0 & 0 & 0 & 0 & 0 & 0 & 0 & 0 & 0 & 0 & 0 & 0 & 0 & 0 & 0 & 0 & 0 & 0 & 0 & 0 & 0 & 0 & 503.977 & 0.0007 \\
\hline I-Pentane & 0 & 0 & 0 & 0 & 0 & 0 & 0 & 0 & 0 & 0 & 0 & 0 & 0 & 0 & 0 & 0 & 0 & 0 & 0 & 0 & 0 & 0 & 894.732 & 0.0008 \\
\hline N-Pentane & 0 & 0 & 0 & 0 & 0 & 0 & 0 & 0 & 0 & 0 & 0 & 0 & 0 & 0 & 0 & 0 & 0 & 0 & 0 & 0 & 0 & 0 & 61.8165 & 0.0008 \\
\hline Trans-2-Pentene & 0 & 0 & 0 & 0 & 0 & 0 & 0 & 0 & 0 & 0 & 0 & 0 & 0 & 0 & 0 & 0 & 0 & 0 & 0 & 0 & 0 & & 107.014 & 0.0008 \\
\hline Cyclopentane & 0 & 0 & 0 & 0 & 0 & 0 & 0 & 0 & 0 & 0 & 0 & 0 & 0 & 0 & 0 & 0 & 0 & 0 & 0 & 0 & 0 & 0 & 0.4545 & 0.0008 \\
\hline 2-2-DiM & 0 & 0 & 0 & 0 & 0 & 0 & 0 & 0 & 0 & 0 & 0 & 0 & 0 & 0 & 0 & 0 & 0 & 0 & 0 & 0 & 0 & 0 & 3.2025 & 0.0009 \\
\hline 2-3-DiMth-Butane & 0 & 0 & 0 & 0 & 0 & 0 & 0 & 0 & 0 & 0 & 0 & 0 & 0 & 0 & 0 & 0 & 0 & 0 & 0 & 0 & 0 & 0 & 1.5313 & 0.0009 \\
\hline 2-Meth & 0 & 0 & 0 & 0 & 0 & 0 & 0 & 0 & 0 & 0 & 0 & 0 & 0 & 0 & 0 & 0 & 0 & 0 & 0 & 0 & 0 & 0 & 2.1139 & 0.0009 \\
\hline 3-Methy & 0 & 0 & 0 & 0 & 0 & 0 & 0 & 0 & 0 & 0 & 0 & 0 & 0 & 0 & 0 & 0 & 0 & 0 & 0 & 0 & 0 & 0 & 1.0266 & 0.0009 \\
\hline MthCycl & 0 & 0 & 0 & 0 & 0 & 0 & 0 & 0 & 0 & 0 & 0 & 0 & 0 & 0 & 0 & 0 & 0 & 0 & 0 & 0 & 0 & 0 & 0.2127 & 0.0009 \\
\hline 2-4-DiMthPentane & 0 & 0 & 0 & 0 & 0 & 0 & 0 & 0 & 0 & 0 & 0 & 0 & 0 & 0 & 0 & 0 & 0 & 0 & 0 & 0 & 0 & 0 & 0.1173 & 0.001 \\
\hline Cyclohe & 0 & 0 & 0 & 0 & 0 & 0 & 0 & 0 & 0 & 0 & 0 & 0 & 0 & 0 & 0 & 0 & 0 & 0 & 0 & 0 & 0 & 0 & 0.0701 & 0.0009 \\
\hline 2-Methyl & 0 & 0 & 0 & 0 & 0 & 0 & 0 & 0 & 0 & 0 & 0 & 0 & 0 & 0 & 0 & 0 & 0 & 0 & 0 & 0 & 0 & 0 & 0.0557 & 0.001 \\
\hline 3-Methylhex & 0 & 0 & 0 & 0 & 0 & 0 & 0 & 0 & 0 & 0 & 0 & 0 & 0 & 0 & 0 & 0 & 0 & 0 & 0 & 0 & 0 & 0 & 0.049 & 0.001 \\
\hline 2-2-4-T & 0 & 0 & 0 & 0 & 0 & 0 & 0 & 0 & 0 & 0 & 0 & 0 & 0 & 0 & 0 & 0 & 0 & 0 & 0 & 0 & 0 & 0 & 0.0297 & 0.001 \\
\hline MthCycl & 0 & 0 & 0 & 0 & 0 & 0 & 0 & 0 & 0 & 0 & 0 & 0 & 0 & 0 & 0 & 0 & 0 & 0 & 0 & 0 & 0 & 0 & 0.0268 & 0.001 \\
\hline 33-2Mth-1-Butene & 0 & 0 & 0 & 0 & 0 & 0 & 0 & 0 & 0 & 0 & 0 & 0 & 0 & 0 & 0 & 0 & 0 & 0 & 0 & 0 & 0 & 0 & 7.9805 & 0.0009 \\
\hline Dimethyl E & 0 & 0 & 0 & 0 & 0 & 0 & 0 & 0 & 0 & 0 & 0 & 0 & 0 & 0 & 0 & 0 & 0 & 0 & 0 & 0 & 0 & 0 & 0 & \\
\hline Toluene & 0 & 0 & 0 & 0 & 0 & 0 & 0 & 0 & 0 & 0 & 0 & 0 & 0 & 0 & 0 & 0 & 0 & 0 & 0 & 0 & 0 & 0 & 0.0783 & 0.001 \\
\hline o-xyle & 0 & 0 & 0 & 0 & 0 & 0 & 0 & 0 & 0 & 0 & 0 & 0 & 0 & 0 & 0 & 0 & 0 & 0 & 0 & 0 & 0 & 0 & 0.0019 & 0.0011 \\
\hline M-Xyle & 0 & 0 & 0 & 0 & 0 & 0 & 0 & 0 & 0 & 0 & 0 & 0 & 0 & 0 & 0 & 0 & 0 & 0 & 0 & 0 & 0 & 0 & 0.007 & 0.0011 \\
\hline P-) & 0 & 0 & 0 & 0 & 0 & 0 & 0 & 0 & 0 & 0 & 0 & 0 & 0 & 0 & 0 & 0 & 0 & 0 & 0 & 0 & 0 & 0 & 0.0035 & 0.0011 \\
\hline M-Eth & 0 & 0 & 0 & 0 & 0 & 0 & 0 & 0 & 0 & 0 & 0 & 0 & 0 & 0 & 0 & 0 & 0 & : & 0 & 0 & 0 & 0 & 0.0002 & \\
\hline $1-2-4$ & 0 & 0 & 0 & 0 & 0 & 0 & 0 & 0 & 0 & 0 & 0 & 0 & 0 & 0 & 0 & 0 & 0 & 0 & 0 & 0 & 0 & 0 & 0.0003 & 0 \\
\hline $2-M$ & 0 & 0 & 0 & 0 & 0 & 0 & 0 & 0 & 0 & 0 & 0 & 0 & 0 & 0 & 0 & 0 & 0 & ～ & 0 & 0 & 0 & 0 & 0.0059 & 0.0012 \\
\hline $1-2$ & 0 & 0 & 0 & 0 & 0 & 0 & 0 & 0 & 0 & 0 & 0 & 0 & 0 & 0 & 0 & 0 & 0 & e & 0 & 0 & 0 & 0 & 0 & \\
\hline $23-2$ & 0 & 0 & 0 & 0 & 0 & 0 & 0 & 0 & 0 & 0 & 0 & 0 & 0 & 0 & 0 & 0 & 0 & 0 & 0 & 0 & 0 & 0 & 0.8467 & 0.0009 \\
\hline $23-2$ & 0 & 0 & 0 & 0 & 0 & 0 & 0 & 0 & 0 & 0 & 0 & 0 & 0 & 0 & 0 & 0 & 0 & 0 & 0 & 0 & 0 & 0 & 0.2105 & 0.0009 \\
\hline 3-Meth & 0 & 0 & 0 & 0 & 0 & 0 & 0 & 0 & 0 & 0 & 0 & 0 & 0 & 0 & 0 & 0 & 0 & 0 & 0 & 0 & 0 & 0 & 0.0037 & 0.001 \\
\hline $2-3-1 \mathrm{~L}$ & 0 & 0 & 0 & 0 & 0 & 0 & 0 & 0 & 0 & 0 & 0 & 0 & 0 & 0 & 0 & 0 & 0 & 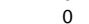 & 0 & 0 & 0 & 0 & 0.0052 & 0.001 \\
\hline 2-3-3-TriMth-C5 & 0 & 0 & 0 & 0 & 0 & 0 & 0 & 0 & 0 & 0 & 0 & 0 & 0 & 0 & 0 & 0 & 0 & 0 & 0 & 0 & 0 & 0 & 0.0066 & 0.001 \\
\hline 1-2-3-4-Tetramet & 0 & 0 & 0 & 0 & 0 & 0 & 0 & 0 & 0 & 0 & 0 & 0 & 0 & 0 & 0 & 0 & 0 & 0 & 0 & 0 & 0 & 0 & 1.0734 & 0.001 \\
\hline
\end{tabular}


Stream No.
Temp F

Pemp F
Ensia
Enth MMBtu/h

Vapor mass fraction

Total lb/h

Flowrates in Ib/

Nitrogen

Argon
Carbon

Carbon
Hydrogen

Carbon Monoxide
Carbon Dioxide

Methane

Acetylene

Ethylene
Ethane

Ethane
Propane

Water
Sulphur

Carbonyl Sulfide
Hydrogen Sulfide

Ammonia

HydrogenChloride

Sllicon Dioxide
Calcium Oxide

Benzene

Naphthalene

Hybrid Poplar Ch

Sulfur Dioxide
Hydrogen Cyanide

Nitric Oxide

Methanol

Ethanol
Propylene

I-Butane

N-Butane

I-Pentane

N-Pentane
Trans-2-Pentene

2-2-DiMth-Butan

2-3-DiMth-Butane

MthCyclopentane

Cyclohexane

2-Methylhexane

3-Methylhexane

thCyclohexan

33-2Mth-1-Butene

Toluen

O-xylene

M-Xylene

P-xylene

M-Ethyltoluene

2-MthNaphthalene

2-4-5-TetMthBz

23-2Mth-1-Butene

23-2Mth-2-Butene

2-3-DiMth-Hexane

2-3-3-TriMth-C5

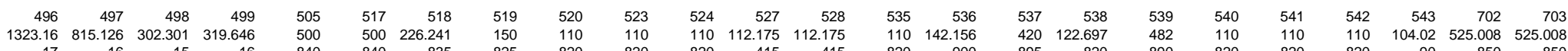

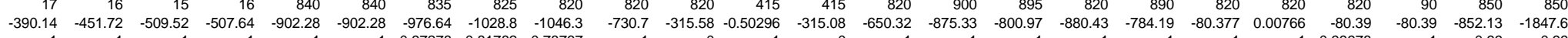

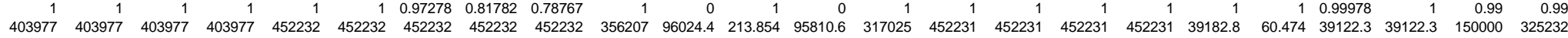

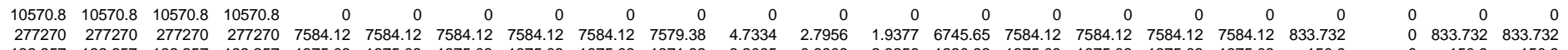
$\begin{array}{lllllllllllllllllll}132.257 & 132.257 & 132.257 & 132.257 & 1375.03 & 1375.03 & 1375.03 & 1375.03 & 1375.03 & 1371.82 & 3.2065 & 0.8808 & 2.3258 & 1220.92 & 1375.03 & 1375.03 & 1375.03 & 1375.03 & 150.9\end{array}$ $\begin{array}{rrrrrrrrrrrrrrrrrrrrrr}0.0011 & 0.0011 & 0.0011 & 0.0011 & 31158.2 & 31158.2 & 31158.2 & 31158.2 & 31158.2 & 31139.8 & 18.3349 & 11.0668 & 7.2681 & 27714.4 & 42990.1 & 42990.1 & 42990.1 & 42990.1 & 342538\end{array}$

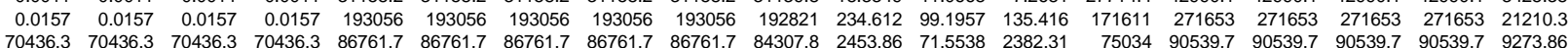
$\begin{array}{lllllllllllllllllllll}0.0002 & 0.0002 & 0.0002 & 0.0002 & 29998.1 & 29998.1 & 29998.1 & 29998.1 & 29998.1 & 29896.8 & 101.324 & 20.9277 & 80.3959 & 26608.1 & 29998.1 & 29998.1 & 29998.1 & 29998.1 & 3288.65\end{array}$ $\begin{array}{rrrrrrrrrrrrrrrrrrr}0.0004 & 0.0004 & 0.0004 & 0.0004 & 0.372 & 0.372 & 0.372 & 0.372 & 0.372 & 0.3668 & 0.0052 & 0.0003 & 0.0049 & 0.3264 & 0.372 & 0.372 & 0.372 & 0.372 & 0.0403 \\ 0.0004 & 0.0004 & 0.0004 & 0.0004 & 0.8136 & 0.8136 & 0.8136 & 0.8136 & 0.8136 & 0.8021 & 0.0115 & 0.0007 & 0.0109 & 0.7139 & 0.8136 & 0.8136 & 0.8136 & 0.8136 & 0.0882 \\ 0\end{array}$ $\begin{array}{rrrrrrrrrrrrrrrrrrrr}0.0006 & 0.0006 & 0.0006 & 0.0006 & 0 & 0 & 0 & 0 & 0 & 0 & 0 & 0 & 0 & 0 & 0 & 0 & 0 & 0 & 0 \\ 45524 & 45524 & 45524 & 45524 & 1579.02 & 1579.02 & 1579.02 & 1579.02 & 1579.02 & 36.5621 & 1542.46 & 0.032 & 1542.42 & 32.5402 & 32.5402 & 32.5402 & 32.5402 & 32.5402 & 4.0218\end{array}$ $0 \quad 150.9 \quad 150.9$ $0.474 \quad 3364.913364 .91$ 0

$\begin{array}{llll}0.0009 & 0.0009 & 0.0009 & 0.0009\end{array}$ $0.0012 \quad 0.0012 \quad 0.0012 \quad 0.0012$

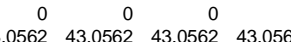

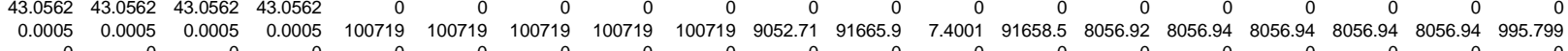
$\begin{array}{rrrr}0.0006 & 0.0006 & 0.0006 & 0.0006\end{array}$ $\begin{array}{lllll}0.0007 & 0.0007 & 0.0007 & 0.0007\end{array}$ $\begin{array}{llll}0.0007 & 0.0007 & 0.0007 & 0.0007 \\ 0.0007 & 0.0007 & 0.0007 & 0.0007 \\ 0.000 & 0.008 & 0.008 & 0.0008\end{array}$ $\begin{array}{llll}0.0008 & 0.0008 & 0.0008 & 0.0008\end{array}$ $\begin{array}{llll}0.0008 & 0.0008 & 0.0008 & 0.0008 \\ 0.0008 & 0.0008 & 0.0008 & 0.0008 \\ 0.0008 & 0.008 & 0.008 & 0.0008\end{array}$ $\begin{array}{llll}0.0008 & 0.0008 & 0.0008 & 0.0008\end{array}$ $\begin{array}{lllll}0.0009 & 0.0009 & 0.0009 & 0.0009\end{array}$ $\begin{array}{llll}0.0009 & 0.0009 & 0.0009 & 0.0009\end{array}$ $\begin{array}{lll}0.0009 & 0.0009 & 0.0009 \\ 0 & 0.0009 & 0.0009\end{array}$ $\begin{array}{llll}0.0009 & 0.0009 & 0.0009 & 0.0009 \\ 0.0009 & 0.0009 & 0.0009 & 0.0009\end{array}$ $\begin{array}{llll}0.0009 & 0.0009 & 0.0009 & 0.0009\end{array}$ $\begin{array}{lllll}0.001 & 0.001 & 0.001 & 0.001\end{array}$ $\begin{array}{llll}0.001 & 0.001 & 0.001 & 0.001\end{array}$ $\begin{array}{llll}0.001 & 0.001 & 0.001 & 0.001 \\ 0.001 & 0.001 & 0.001 & 0.001\end{array}$ $\begin{array}{rrrr}0.001 & 0.001 & 0.001 & 0.001 \\ 0.0009 & 0.0009 & 0.0009 & 0.0009\end{array}$ $\begin{array}{rrrrr}0.001 & 0.001 & 0.001 & 0.001 \\ 0.0011 & 0.0011 & 0.0011 & 0.0011\end{array}$ $\begin{array}{lllll}0.0011 & 0.0011 & 0.0011 & 0.001\end{array}$ $\begin{array}{lllll}0.0011 & 0.0011 & 0.0011 & 0.001 \\ & 0 & 0 & & 0\end{array}$ $\begin{array}{rrrrr}0.0012 & 0.0012 & 0.0012 & 0.0012\end{array}$ $\begin{array}{llll}0.0009 & 0.0009 & 0.0009 & 0.0009\end{array}$ $\begin{array}{rrrr}0.0009 & 0.0009 & 0.0009 & 0.0009\end{array}$ $\begin{array}{llll}0.001 & 0.001 & 0.001 & 0.001 \\ 0.001 & 0.001 & 0.001 & 0.001\end{array}$ $\begin{array}{llll}0.001 & 0.001 & 0.001 & 0.001 \\ 0.001 & 0.001 & 0.001 & 0.001 \\ 0.001 & 0.001 & 0.001 & 0.001\end{array}$

\begin{tabular}{|c|c|c|c|c|c|c|c|c|c|c|c|c|c|c|}
\hline 9.02 & 1579.02 & & & & & & & & & & & & & \\
\hline & 0 & 0 & 0 & & 0 & & 0 & 0 & 0 & 0 & & 0 & 0 & $\begin{array}{l}4.0210 \\
0\end{array}$ \\
\hline 0 & 0 & 0 & 0 & 0 & 0 & 0 & 0 & 0 & 0 & 0 & 0 & 0 & 0 & 0 \\
\hline 0 & 0 & 0 & 0 & 0 & 0 & 0 & 0 & 0 & 0 & 0 & 0 & 0 & 0 & \\
\hline 0 & 0 & 0 & 0 & 0 & 0 & 0 & 0 & 0 & 0 & 0 & 0 & 0 & 0 & 0 \\
\hline 0 & 0 & 0 & 0 & 0 & 0 & 0 & 0 & 0 & 0 & 0 & 0 & 0 & 0 & \\
\hline 0 & 0 & 0 & 0 & 0 & 0 & 0 & 0 & 0 & 0 & 0 & 0 & 0 & 0 & \\
\hline 0 & 0 & 0 & 0 & 0 & 0 & 0 & 0 & 0 & 0 & 0 & 0 & 0 & 0 & 0 \\
\hline 0 & 0 & 0 & 0 & 0 & 0 & 0 & 0 & 0 & 0 & 0 & 0 & 0 & 0 & 0 \\
\hline 0 & 0 & 0 & 0 & 0 & 0 & 0 & 0 & 0 & 0 & 0 & 0 & 0 & 0 & \\
\hline 0 & 0 & 0 & 0 & 0 & 0 & 0 & 0 & 0 & 0 & 0 & 0 & 0 & 0 & \\
\hline 0 & 0 & 0 & 0 & 0 & 0 & 0 & 0 & 0 & 0 & 0 & 0 & 0 & 0 & \\
\hline 0 & 0 & 0 & 0 & 0 & 0 & 0 & 0 & 0 & 0 & 0 & 0 & 0 & 0 & \\
\hline 0 & 0 & 0 & 0 & 0 & 0 & 0 & 0 & 0 & 0 & 0 & 0 & 0 & 0 & \\
\hline 100719 & 100719 & 100719 & 100719 & 100719 & 9052.71 & 91665.9 & 7.4001 & 91658.5 & 8056.92 & 8056.94 & 8056.94 & 8056.94 & 8056.94 & 995.799 \\
\hline & & & 0 & 0 & 0 & 0 & 0 & 0 & 0 & 0 & 0 & & & \\
\hline 0 & 0 & 0 & 0 & 0 & 0 & 0 & 0 & 0 & 0 & 0 & 0 & 0 & 0 & \\
\hline 0 & 0 & 0 & 0 & 0 & 0 & 0 & 0 & 0 & 0 & 0 & 0 & 0 & 0 & \\
\hline 0 & 0 & 0 & 0 & 0 & 0 & 0 & 0 & 0 & 0 & 0 & 0 & 0 & 0 & \\
\hline 0 & 0 & 0 & 0 & 0 & 0 & 0 & 0 & 0 & 0 & 0 & 0 & 0 & 0 & \\
\hline 0 & 0 & 0 & 0 & 0 & 0 & 0 & 0 & 0 & 0 & 0 & 0 & 0 & 0 & \\
\hline 0 & 0 & 0 & 0 & 0 & 0 & 0 & 0 & 0 & 0 & 0 & 0 & 0 & 0 & \\
\hline 0 & 0 & 0 & 0 & 0 & 0 & 0 & 0 & 0 & 0 & 0 & 0 & 0 & 0 & \\
\hline 0 & 0 & 0 & 0 & 0 & 0 & 0 & 0 & 0 & 0 & 0 & 0 & 0 & 0 & \\
\hline 0 & 0 & 0 & 0 & 0 & 0 & 0 & 0 & 0 & 0 & 0 & 0 & 0 & 0 & \\
\hline 0 & 0 & 0 & 0 & 0 & 0 & 0 & 0 & 0 & 0 & 0 & 0 & 0 & 0 & \\
\hline 0 & 0 & 0 & 0 & 0 & 0 & 0 & 0 & 0 & 0 & 0 & 0 & 0 & 0 & \\
\hline 0 & 0 & 0 & 0 & 0 & 0 & 0 & 0 & 0 & 0 & 0 & 0 & 0 & 0 & \\
\hline 0 & 0 & 0 & 0 & 0 & 0 & 0 & 0 & 0 & 0 & 0 & 0 & 0 & 0 & \\
\hline 0 & 0 & 0 & 0 & 0 & 0 & 0 & 0 & 0 & 0 & 0 & 0 & 0 & 0 & \\
\hline 0 & 0 & 0 & 0 & 0 & 0 & 0 & 0 & 0 & 0 & 0 & 0 & 0 & 0 & \\
\hline 0 & 0 & 0 & 0 & 0 & 0 & 0 & 0 & 0 & 0 & 0 & 0 & 0 & 0 & \\
\hline 0 & 0 & 0 & 0 & 0 & 0 & 0 & 0 & 0 & 0 & 0 & 0 & 0 & 0 & \\
\hline 0 & 0 & 0 & 0 & 0 & 0 & 0 & 0 & 0 & 0 & 0 & 0 & 0 & 0 & \\
\hline 0 & 0 & 0 & 0 & 0 & 0 & 0 & 0 & 0 & 0 & 0 & 0 & 0 & 0 & \\
\hline 0 & 0 & 0 & 0 & 0 & 0 & 0 & 0 & 0 & 0 & 0 & 0 & 0 & 0 & \\
\hline 0 & 0 & 0 & 0 & 0 & 0 & 0 & 0 & 0 & 0 & 0 & 0 & 0 & 0 & \\
\hline 0 & 0 & 0 & 0 & 0 & 0 & 0 & 0 & 0 & 0 & 0 & 0 & 0 & 0 & \\
\hline 0 & 0 & 0 & 0 & 0 & 0 & 0 & 0 & 0 & 0 & 0 & 0 & 0 & 0 & \\
\hline 0 & 0 & 0 & 0 & 0 & 0 & 0 & 0 & 0 & 0 & 0 & 0 & 0 & 0 & \\
\hline 0 & 0 & 0 & 0 & 0 & 0 & 0 & 0 & 0 & 0 & 0 & 0 & 0 & 0 & \\
\hline 0 & 0 & 0 & 0 & 0 & 0 & 0 & 0 & 0 & 0 & 0 & 0 & 0 & 0 & \\
\hline 0 & 0 & 0 & 0 & 0 & 0 & 0 & 0 & 0 & 0 & 0 & 0 & 0 & 0 & \\
\hline 0 & 0 & 0 & 0 & 0 & 0 & 0 & 0 & 0 & 0 & 0 & 0 & 0 & 0 & \\
\hline 0 & 0 & 0 & 0 & 0 & 0 & 0 & 0 & 0 & 0 & 0 & 0 & 0 & 0 & \\
\hline 0 & 0 & 0 & 0 & 0 & 0 & 0 & 0 & 0 & 0 & 0 & 0 & 0 & 0 & \\
\hline 0 & 0 & 0 & 0 & 0 & 0 & 0 & 0 & 0 & 0 & 0 & 0 & 0 & 0 & \\
\hline c & 0 & 0 & 0 & 0 & 0 & 0 & 0 & 0 & 0 & 0 & 0 & 0 & 0 & \\
\hline & 0 & 0 & 0 & 0 & 0 & 0 & 0 & 0 & 0 & 0 & 0 & 0 & 0 & \\
\hline & 0 & 0 & 0 & 0 & 0 & U & 0 & 0 & 0 & 0 & 0 & 0 & 0 & \\
\hline 0 & 0 & 0 & 0 & 0 & 0 & 0 & 0 & 0 & 0 & 0 & 0 & 0 & 0 & \\
\hline
\end{tabular}

$\begin{array}{lrrr}0 & 9273.86 & 9273.86 \\ 0 & 3288.65 & 32886\end{array}$ $\begin{array}{lll}0 & 0.0882 & 0.0882 \\ 0 & 0.028 & 0\end{array}$ $\begin{array}{rrrrr}0 & 0 & 0 & 0 & 0 \\ 0 & 4.0218 & 4.0218 & 150000 & 325232\end{array}$ $\begin{array}{lll}0 & 0 & 0\end{array}$ 


\begin{tabular}{|c|c|c|c|c|c|c|c|c|c|c|c|c|c|c|c|c|c|c|c|c|c|c|c|c|}
\hline Stream No. & & 706 & 707 & 708 & 710 & 712 & 715 & 718 & 719 & 720 & 721 & 725 & 731 & & 734 & 736 & 741 & 745 & 751 & 761 & 762 & 770 & 771 & 773 \\
\hline Temp F & 525.008 & 525.008 & 525.008 & 525.008 & 525.008 & 525.006 & 175.579 & 525.006 & 200 & 900 & 900 & 899.968 & 743.513 & 743.513 & 743.513 & 743.513 & 287 & 287 & 116.755 & 116.755 & 116.882 & 60 & 72.9587 & 456 \\
\hline Pres psia & 850 & 850 & 850 & 850 & 850 & 850 & 35 & 850 & 850 & 850 & 849 & 849 & 450 & 450 & 450 & 450 & 35 & 35 & 15 & 1.5 & & 60 & 60 & 450 \\
\hline Enth MMBtu/h & -2742 & -2711.3 & -1349.5 & -1361.8 & -30.665 & 0 & 0 & -30.665 & -32.371 & -1288.5 & -1300.2 & -2588.7 & -2622.1 & -99.201 & -106.7 & -2416.2 & -2505.3 & -165 & -2408.9 & -2791.2 & -2791.2 & -901.18 & -899.47 & -116.27 \\
\hline Vapor mass fraction & 0.98999 & & & & 0 & 1 & 1 & & 0 & 1 & & & 1 & & 1 & & & 1 & 0.90484 & $1.00 \mathrm{E}-06$ & & & & \\
\hline Total lb/h & 482669 & 477839 & 237839 & 240000 & 4829.71 & 0 & 0 & 4829.71 & 4829.71 & 237840 & 240000 & 477840 & 477840 & 18078 & 19444 & 440318 & 440318 & 29000 & 411318 & 411318 & 411318 & 131697 & 131697 & 18078 \\
\hline Flowrates in Ib/h & & & & & & & & & & & & & & & & & & & & & & & & \\
\hline Oxygen & 0 & 0 & 0 & 0 & 0 & 0 & 0 & 0 & 0 & 0 & 0 & 0 & 0 & 0 & 0 & 0 & 0 & 0 & 0 & 0 & 0 & 0 & 0 & \\
\hline Nitrogen & 0 & 0 & 0 & 0 & 0 & 0 & 0 & 0 & 0 & 0 & 0 & 0 & 0 & 0 & 0 & 0 & 0 & 0 & 0 & 0 & 0 & 0 & 0 & 0 \\
\hline Argon & 0 & 0 & 0 & 0 & 0 & 0 & 0 & 0 & 0 & 0 & 0 & 0 & 0 & 0 & 0 & 0 & 0 & 0 & 0 & 0 & 0 & 0 & 0 & 0 \\
\hline Carbon & 0 & 0 & 0 & 0 & 0 & 0 & 0 & 0 & 0 & 0 & 0 & 0 & 0 & 0 & 0 & 0 & 0 & 0 & 0 & 0 & 0 & 0 & 0 & 0 \\
\hline Hydrogen & 0 & 0 & 0 & 0 & 0 & 0 & 0 & 0 & 0 & 0 & 0 & 0 & 0 & 0 & 0 & 0 & 0 & 0 & 0 & 0 & 0 & 0 & 0 & \\
\hline Carbon Monoxide & 0 & 0 & 0 & 0 & 0 & 0 & 0 & 0 & 0 & 0 & 0 & 0 & 0 & 0 & 0 & 0 & 0 & 0 & 0 & 0 & 0 & 0 & 0 & \\
\hline Carbon Dioxide & 0 & 0 & 0 & 0 & 0 & 0 & 0 & 0 & 0 & 0 & 0 & 0 & 0 & 0 & 0 & 0 & 0 & 0 & 0 & 0 & 0 & 0 & 0 & \\
\hline Methane & 0 & 0 & 0 & 0 & 0 & 0 & 0 & 0 & 0 & 0 & 0 & 0 & 0 & 0 & 0 & 0 & 0 & 0 & 0 & 0 & 0 & 0 & 0 & \\
\hline Acetylene & 0 & 0 & 0 & 0 & 0 & 0 & 0 & 0 & 0 & 0 & 0 & 0 & 0 & 0 & 0 & 0 & 0 & 0 & 0 & 0 & 0 & 0 & 0 & \\
\hline Ethylene & 0 & 0 & 0 & 0 & 0 & 0 & 0 & 0 & 0 & 0 & 0 & 0 & 0 & 0 & 0 & 0 & 0 & 0 & 0 & 0 & 0 & 0 & 0 & \\
\hline Ethane & 0 & 0 & 0 & 0 & 0 & 0 & 0 & 0 & 0 & 0 & 0 & 0 & 0 & 0 & 0 & 0 & 0 & 0 & 0 & 0 & 0 & 0 & 0 & \\
\hline Propane & 0 & 0 & 0 & 0 & 0 & 0 & 0 & 0 & 0 & 0 & 0 & 0 & 0 & 0 & 0 & 0 & 0 & 0 & 0 & 0 & 0 & 0 & 0 & 0 \\
\hline Water & 482669 & 477839 & 237839 & 240000 & 4829.71 & 0 & 0 & 4829.71 & 4829.71 & 237840 & 240000 & 477840 & 477840 & 18078 & 19444 & 440318 & 440318 & 29000 & 411318 & 411318 & 411318 & 131697 & 131697 & 18078 \\
\hline Sulphur & & & 0 & 0 & 0 & 0 & 0 & 0 & 0 & & & 0 & 0 & 0 & 0 & & 0 & 0 & & & 0 & 0 & 0 & \\
\hline Carbonyl Sulfide & 0 & 0 & 0 & 0 & 0 & 0 & 0 & 0 & 0 & 0 & 0 & 0 & 0 & 0 & 0 & 0 & 0 & 0 & 0 & 0 & 0 & 0 & 0 & 0 \\
\hline Hydrogen Sulfide & 0 & 0 & 0 & 0 & 0 & 0 & 0 & 0 & 0 & 0 & 0 & 0 & 0 & 0 & 0 & 0 & 0 & 0 & 0 & 0 & 0 & 0 & 0 & \\
\hline Ammonia & 0 & 0 & 0 & 0 & 0 & 0 & 0 & 0 & 0 & 0 & 0 & 0 & 0 & 0 & 0 & 0 & 0 & 0 & 0 & 0 & 0 & 0 & 0 & \\
\hline HydrogenChloride & 0 & 0 & 0 & 0 & 0 & 0 & 0 & 0 & 0 & 0 & 0 & 0 & 0 & 0 & 0 & 0 & 0 & 0 & 0 & 0 & 0 & 0 & 0 & \\
\hline Silicon Dioxide & 0 & 0 & 0 & 0 & 0 & 0 & 0 & 0 & 0 & 0 & 0 & 0 & 0 & 0 & 0 & 0 & 0 & 0 & 0 & 0 & 0 & 0 & 0 & \\
\hline Calcium Oxide & 0 & 0 & 0 & 0 & 0 & 0 & 0 & 0 & 0 & 0 & 0 & 0 & 0 & 0 & 0 & 0 & 0 & 0 & 0 & 0 & 0 & 0 & 0 & \\
\hline Benzene & 0 & 0 & 0 & 0 & 0 & 0 & 0 & 0 & 0 & 0 & 0 & 0 & 0 & 0 & 0 & 0 & 0 & 0 & 0 & 0 & 0 & 0 & 0 & \\
\hline Naphthalene & 0 & 0 & 0 & 0 & 0 & 0 & 0 & 0 & 0 & 0 & 0 & 0 & 0 & 0 & 0 & 0 & 0 & 0 & 0 & 0 & 0 & 0 & 0 & \\
\hline Hybrid Poplar Ch & 0 & 0 & 0 & 0 & 0 & 0 & 0 & 0 & 0 & 0 & 0 & 0 & 0 & 0 & 0 & 0 & 0 & 0 & 0 & 0 & 0 & 0 & 0 & \\
\hline Sulfur Dioxide & 0 & 0 & 0 & 0 & 0 & 0 & 0 & 0 & 0 & 0 & 0 & 0 & 0 & 0 & 0 & 0 & 0 & 0 & 0 & 0 & 0 & 0 & 0 & \\
\hline Hydrogen Cyanide & 0 & 0 & 0 & 0 & 0 & 0 & 0 & 0 & 0 & 0 & 0 & 0 & 0 & 0 & 0 & 0 & 0 & 0 & 0 & 0 & 0 & 0 & 0 & \\
\hline Nitric Oxide & 0 & 0 & 0 & 0 & 0 & 0 & 0 & 0 & 0 & 0 & 0 & 0 & 0 & 0 & 0 & 0 & 0 & 0 & 0 & 0 & 0 & 0 & 0 & \\
\hline Methanol & 0 & 0 & 0 & 0 & 0 & 0 & 0 & 0 & 0 & 0 & 0 & 0 & 0 & 0 & 0 & 0 & 0 & 0 & 0 & 0 & 0 & 0 & 0 & \\
\hline Ethanol & 0 & 0 & 0 & 0 & 0 & 0 & 0 & 0 & 0 & 0 & 0 & 0 & 0 & 0 & 0 & 0 & 0 & 0 & 0 & 0 & 0 & 0 & 0 & \\
\hline Propylene & 0 & 0 & 0 & 0 & 0 & 0 & 0 & 0 & 0 & 0 & 0 & 0 & 0 & 0 & 0 & 0 & 0 & 0 & 0 & 0 & 0 & 0 & 0 & \\
\hline I-Butane & 0 & 0 & 0 & 0 & 0 & 0 & 0 & 0 & 0 & 0 & 0 & 0 & 0 & 0 & 0 & 0 & 0 & 0 & 0 & 0 & 0 & 0 & 0 & \\
\hline N-Butane & 0 & 0 & 0 & 0 & 0 & 0 & 0 & 0 & 0 & 0 & 0 & 0 & 0 & 0 & 0 & 0 & 0 & 0 & 0 & 0 & 0 & 0 & 0 & \\
\hline Cis-2-Butene & 0 & 0 & 0 & 0 & 0 & 0 & 0 & 0 & 0 & 0 & 0 & 0 & 0 & 0 & 0 & 0 & 0 & 0 & 0 & 0 & 0 & 0 & 0 & \\
\hline I-Pentan & 0 & 0 & 0 & 0 & 0 & 0 & 0 & 0 & 0 & 0 & 0 & 0 & 0 & 0 & 0 & 0 & 0 & 0 & 0 & 0 & 0 & 0 & 0 & \\
\hline N-Pentane & 0 & 0 & 0 & 0 & 0 & 0 & 0 & 0 & 0 & 0 & 0 & 0 & 0 & 0 & 0 & 0 & 0 & 0 & 0 & 0 & 0 & 0 & 0 & \\
\hline Trans-2-Pentene & 0 & 0 & 0 & 0 & 0 & 0 & 0 & 0 & 0 & 0 & 0 & 0 & 0 & 0 & 0 & 0 & 0 & 0 & 0 & 0 & 0 & 0 & 0 & \\
\hline Cyclopentane & 0 & 0 & 0 & 0 & 0 & 0 & 0 & 0 & 0 & 0 & 0 & 0 & 0 & 0 & 0 & 0 & 0 & 0 & 0 & 0 & 0 & 0 & 0 & 0 \\
\hline 2-2-DiMth-Butane & 0 & 0 & 0 & 0 & 0 & 0 & 0 & 0 & 0 & 0 & 0 & 0 & 0 & 0 & 0 & 0 & 0 & 0 & 0 & 0 & 0 & 0 & 0 & \\
\hline 2-3-DiMth-Butane & 0 & 0 & 0 & 0 & 0 & 0 & 0 & 0 & 0 & 0 & 0 & 0 & 0 & 0 & 0 & 0 & 0 & 0 & 0 & 0 & 0 & 0 & 0 & 0 \\
\hline 2-Methylpentane & 0 & 0 & 0 & 0 & 0 & 0 & 0 & 0 & 0 & 0 & 0 & 0 & 0 & 0 & 0 & 0 & 0 & 0 & 0 & 0 & 0 & 0 & 0 & 0 \\
\hline 3-Methylpentane & 0 & 0 & 0 & 0 & 0 & 0 & 0 & 0 & 0 & 0 & 0 & 0 & 0 & 0 & 0 & 0 & 0 & 0 & 0 & 0 & 0 & 0 & 0 & 0 \\
\hline MthCyclopentane & 0 & 0 & 0 & 0 & 0 & 0 & 0 & 0 & 0 & 0 & 0 & 0 & 0 & 0 & 0 & 0 & 0 & 0 & 0 & 0 & 0 & 0 & 0 & 0 \\
\hline 2-4-DiMthPentane & 0 & 0 & 0 & 0 & 0 & 0 & 0 & 0 & 0 & 0 & 0 & 0 & 0 & 0 & 0 & 0 & 0 & 0 & 0 & 0 & 0 & 0 & 0 & 0 \\
\hline Cyclohexane & 0 & 0 & 0 & 0 & 0 & 0 & 0 & 0 & 0 & 0 & 0 & 0 & 0 & 0 & 0 & 0 & 0 & 0 & 0 & 0 & 0 & 0 & 0 & 0 \\
\hline 2-Methylhexane & 0 & 0 & 0 & 0 & 0 & 0 & 0 & 0 & 0 & 0 & 0 & 0 & 0 & 0 & 0 & 0 & 0 & 0 & 0 & 0 & 0 & 0 & 0 & 0 \\
\hline 3-Methylhexane & 0 & 0 & 0 & 0 & 0 & 0 & 0 & 0 & 0 & 0 & 0 & 0 & 0 & 0 & 0 & 0 & 0 & 0 & 0 & 0 & 0 & 0 & 0 & 0 \\
\hline 2-2-4-TriMth-C5 & 0 & 0 & 0 & 0 & 0 & 0 & 0 & 0 & 0 & 0 & 0 & 0 & 0 & 0 & 0 & 0 & 0 & 0 & 0 & 0 & 0 & 0 & 0 & 0 \\
\hline lexane & 0 & 0 & 0 & 0 & 0 & 0 & 0 & 0 & 0 & 0 & 0 & 0 & 0 & 0 & 0 & 0 & 0 & 0 & 0 & 0 & 0 & 0 & 0 & 0 \\
\hline Butene & 0 & 0 & 0 & 0 & 0 & 0 & 0 & 0 & 0 & 0 & 0 & 0 & 0 & 0 & 0 & 0 & 0 & 0 & 0 & 0 & 0 & 0 & 0 & 0 \\
\hline Dimethyl Ether & 0 & 0 & 0 & 0 & 0 & 0 & 0 & 0 & 0 & 0 & 0 & 0 & 0 & 0 & 0 & 0 & 0 & 0 & 0 & 0 & 0 & 0 & 0 & 0 \\
\hline Toluene & 0 & 0 & 0 & 0 & 0 & 0 & 0 & 0 & 0 & 0 & 0 & 0 & 0 & 0 & 0 & 0 & 0 & 0 & 0 & 0 & 0 & 0 & 0 & 0 \\
\hline O-Xylene & 0 & 0 & 0 & 0 & 0 & 0 & 0 & 0 & 0 & 0 & 0 & 0 & 0 & 0 & 0 & 0 & 0 & 0 & 0 & 0 & 0 & 0 & 0 & 0 \\
\hline$M-X y$ & 0 & 0 & 0 & 0 & 0 & 0 & 0 & 0 & 0 & 0 & 0 & 0 & 0 & 0 & 0 & 0 & 0 & 0 & 0 & 0 & 0 & 0 & 0 & 0 \\
\hline & 0 & 0 & 0 & 0 & 0 & 0 & 0 & 0 & 0 & 0 & 0 & 0 & 0 & 0 & 0 & 0 & 0 & 0 & 0 & 0 & 0 & 0 & 0 & 0 \\
\hline Iuene & 0 & 0 & 0 & 0 & 0 & 0 & 0 & 0 & 0 & 0 & 0 & 0 & 0 & 0 & 0 & 0 & 0 & 0 & 0 & 0 & 0 & 0 & 0 & 0 \\
\hline & 0 & 0 & 0 & 0 & 0 & 0 & 0 & 0 & 0 & 0 & 0 & 0 & 0 & 0 & 0 & 0 & 0 & 0 & 0 & 0 & 0 & 0 & 0 & 0 \\
\hline 2-Mt & 0 & 0 & 0 & 0 & 0 & 0 & 0 & 0 & 0 & 0 & 0 & 0 & 0 & 0 & 0 & 0 & 0 & 0 & 0 & 0 & 0 & 0 & 0 & 0 \\
\hline & 0 & 0 & 0 & 0 & 0 & 0 & 0 & 0 & 0 & 0 & 0 & 0 & 0 & 0 & 0 & 0 & 0 & 0 & 0 & 0 & 0 & 0 & 0 & 0 \\
\hline 23- & 0 & 0 & 0 & 0 & 0 & 0 & 0 & 0 & 0 & 0 & 0 & 0 & 0 & 0 & 0 & 0 & 0 & 0 & 0 & 0 & 0 & 0 & 0 & 0 \\
\hline $23-$ & 0 & 0 & 0 & 0 & 0 & 0 & 0 & 0 & 0 & 0 & 0 & 0 & 0 & 0 & 0 & 0 & 0 & 0 & 0 & 0 & 0 & 0 & 0 & 0 \\
\hline $3-N$ & 0 & 0 & 0 & 0 & 0 & 0 & 0 & 0 & 0 & 0 & 0 & 0 & 0 & 0 & 0 & 0 & 0 & 0 & 0 & 0 & 0 & 0 & 0 & 0 \\
\hline 2-3-DiMth-Hexane & 0 & 0 & 0 & 0 & 0 & 0 & 0 & 0 & 0 & 0 & 0 & 0 & 0 & 0 & 0 & 0 & 0 & 0 & 0 & 0 & 0 & 0 & 0 & 0 \\
\hline 2-3-3-TriMth-C5 & 0 & 0 & 0 & 0 & 0 & 0 & 0 & 0 & 0 & 0 & 0 & 0 & 0 & 0 & 0 & 0 & 0 & 0 & 0 & 0 & 0 & 0 & 0 & \\
\hline 1-2-3-4-Tetramet & 0 & 0 & 0 & 0 & 0 & 0 & 0 & 0 & 0 & 0 & 0 & 0 & 0 & 0 & 0 & 0 & 0 & 0 & 0 & 0 & 0 & 0 & 0 & \\
\hline
\end{tabular}




\begin{tabular}{|c|c|c|c|c|c|c|c|c|c|c|c|c|c|c|c|c|c|c|c|c|c|c|c|c|}
\hline Stream No. & 1850 & 1851 & 1929 & 1930 & 1931 & 1935 & 1936 & 1938 & 1940 & 1941 & 1942 & 1943 & 1944 & 1945 & 1947 & 1948 & 1949 & 1950 & 1952 & 1953 & 1954 & 1955 & 1956 & 1980 \\
\hline Temp F & 90 & 110 & 456.586 & 456.588 & 456.588 & 181 & 400 & 525.008 & 715 & 456.586 & 456.586 & 456.588 & 525.01 & 900 & 525.01 & 900 & 178 & 525.008 & 456.584 & 456.586 & 456.586 & 715 & 456.586 & 456.586 \\
\hline Pres psia & 65 & 60 & 450 & & 450 & 850 & 850 & 850 & 450 & 450 & 450 & 450 & 850 & 850 & 850 & 849 & 850 & 850 & 450 & 450 & 450 & 450 & 450 & 450 \\
\hline Enth MMBtu/h & -5954.5 & -5937 & -236.59 & -236.59 & -236.59 & -1008.3 & -974.17 & -852.13 & -789.81 & -23.333 & -24.494 & -315.55 & -1349.5 & -1288.5 & -1361.8 & -1300.2 & -2187.1 & -1847.6 & -1003.3 & -885.23 & -630.21 & -99.484 & -116.26 & -56.499 \\
\hline Vapor mass fraction & 0 & & & & & & 0 & 0.99 & & 0.39969 & 0.001 & & & & & & & 0.99 & & 0.98994 & & & $1.00 \mathrm{E}-06$ & \\
\hline $\begin{array}{l}\text { Total lb/h } \\
\text { Flowrates in lb/h }\end{array}$ & 874013 & 874013 & 41754 & 41753.8 & 41753.8 & 150000 & 150000 & 150000 & 143523 & 3809.25 & 3809.25 & 55688 & 237840 & 237840 & 240000 & 240000 & 325232 & 325232 & 156014 & 156014 & 111220 & 18078.2 & 18078.2 & 9971 \\
\hline $\begin{array}{l}\text { Flowrates in lb/h } \\
\text { Oxygen }\end{array}$ & 0 & 0 & 0 & 0 & 0 & 0 & 0 & 0 & 0 & 0 & 0 & 0 & 0 & 0 & 0 & 0 & 0 & 0 & 0 & 0 & 0 & 0 & 0 & 0 \\
\hline Nitrogen & 0 & 0 & 0 & 0 & 0 & 0 & 0 & 0 & 0 & 0 & 0 & 0 & 0 & 0 & 0 & 0 & 0 & 0 & 0 & 0 & 0 & 0 & 0 & 0 \\
\hline Argon & 0 & 0 & 0 & 0 & 0 & 0 & 0 & 0 & 0 & 0 & 0 & 0 & 0 & 0 & 0 & 0 & 0 & 0 & 0 & 0 & 0 & 0 & 0 & \\
\hline Carbon & 0 & 0 & 0 & 0 & 0 & 0 & 0 & 0 & 0 & 0 & 0 & 0 & 0 & 0 & 0 & 0 & 0 & 0 & 0 & 0 & 0 & 0 & 0 & \\
\hline Hydrogen & 0 & 0 & 0 & 0 & 0 & 0 & 0 & 0 & 0 & 0 & 0 & 0 & 0 & 0 & 0 & 0 & 0 & 0 & 0 & 0 & 0 & 0 & 0 & \\
\hline Carbon Monoxide & 0 & 0 & 0 & 0 & 0 & 0 & 0 & 0 & 0 & 0 & 0 & 0 & 0 & 0 & 0 & 0 & 0 & 0 & 0 & 0 & 0 & 0 & 0 & \\
\hline Carbon Dioxide & 0 & 0 & 0 & 0 & 0 & 0 & 0 & 0 & 0 & 0 & 0 & 0 & 0 & 0 & 0 & 0 & 0 & 0 & 0 & 0 & 0 & 0 & 0 & \\
\hline Methane & 0 & 0 & 0 & 0 & 0 & 0 & 0 & 0 & 0 & 0 & 0 & 0 & 0 & 0 & 0 & 0 & 0 & 0 & 0 & 0 & 0 & 0 & 0 & 0 \\
\hline Acetylene & 0 & 0 & 0 & 0 & 0 & 0 & 0 & 0 & 0 & 0 & 0 & 0 & 0 & 0 & 0 & 0 & 0 & 0 & 0 & 0 & 0 & 0 & 0 & \\
\hline Ethylene & 0 & 0 & 0 & 0 & 0 & 0 & 0 & 0 & 0 & 0 & 0 & 0 & 0 & 0 & 0 & 0 & 0 & 0 & 0 & 0 & 0 & 0 & 0 & \\
\hline Ethane & 0 & 0 & 0 & 0 & 0 & 0 & 0 & 0 & 0 & 0 & 0 & 0 & 0 & 0 & 0 & 0 & 0 & 0 & 0 & 0 & 0 & 0 & 0 & 0 \\
\hline Propane & & & 0 & & & 0 & 0 & 0 & & 0 & 0 & 0 & 0 & 0 & 0 & 0 & 0 & 0 & 0 & 0 & 0 & 0 & 0 & 0 \\
\hline Water & 874013 & 874013 & 41754 & 41753.8 & 41753.8 & 150000 & 150000 & 150000 & 143523 & 3809.25 & 3809.25 & 55688 & 237840 & 237840 & 240000 & 240000 & 325232 & 325232 & 156014 & 156014 & 111220 & 18078.2 & 18078.2 & 9971 \\
\hline Sulphur & & & 0 & 0 & 0 & 0 & & 0 & 0 & 0 & 0 & 0 & 0 & 0 & 0 & 0 & 0 & 0 & 0 & & 0 & 0 & 0 & 0 \\
\hline Carbonyl Sulfide & 0 & 0 & 0 & 0 & 0 & 0 & 0 & 0 & 0 & 0 & 0 & 0 & 0 & 0 & 0 & 0 & 0 & 0 & 0 & 0 & 0 & 0 & 0 & 0 \\
\hline Hydrogen Sulfide & 0 & 0 & 0 & 0 & 0 & 0 & 0 & 0 & 0 & 0 & 0 & 0 & 0 & 0 & 0 & 0 & 0 & 0 & 0 & 0 & 0 & 0 & 0 & 0 \\
\hline Ammonia & 0 & 0 & 0 & 0 & 0 & 0 & 0 & 0 & 0 & 0 & 0 & 0 & 0 & 0 & 0 & 0 & 0 & 0 & 0 & 0 & 0 & 0 & 0 & 0 \\
\hline HydrogenChloride & 0 & 0 & 0 & 0 & 0 & 0 & 0 & 0 & 0 & 0 & 0 & 0 & 0 & 0 & 0 & 0 & 0 & 0 & 0 & 0 & 0 & 0 & 0 & 0 \\
\hline Silicon Dioxide & 0 & 0 & 0 & 0 & 0 & 0 & 0 & 0 & 0 & 0 & 0 & 0 & 0 & 0 & 0 & 0 & 0 & 0 & 0 & 0 & 0 & 0 & 0 & 0 \\
\hline Calcium Oxide & 0 & 0 & 0 & 0 & 0 & 0 & 0 & 0 & 0 & 0 & 0 & 0 & 0 & 0 & 0 & 0 & 0 & 0 & 0 & 0 & 0 & 0 & 0 & 0 \\
\hline Benzene & 0 & 0 & 0 & 0 & 0 & 0 & 0 & 0 & 0 & 0 & 0 & 0 & 0 & 0 & 0 & 0 & 0 & 0 & 0 & 0 & 0 & 0 & 0 & 0 \\
\hline Naphthalene & 0 & 0 & 0 & 0 & 0 & 0 & 0 & 0 & 0 & 0 & 0 & 0 & 0 & 0 & 0 & 0 & 0 & 0 & 0 & 0 & 0 & 0 & 0 & 0 \\
\hline Hybrid Poplar Ch & 0 & 0 & 0 & 0 & 0 & 0 & 0 & 0 & 0 & 0 & 0 & 0 & 0 & 0 & 0 & 0 & 0 & 0 & 0 & 0 & 0 & 0 & 0 & 0 \\
\hline Sulfur Dioxide & 0 & 0 & 0 & 0 & 0 & 0 & 0 & 0 & 0 & 0 & 0 & 0 & 0 & 0 & 0 & 0 & 0 & 0 & 0 & 0 & 0 & 0 & 0 & 0 \\
\hline Hydrogen Cyanide & 0 & 0 & 0 & 0 & 0 & 0 & 0 & 0 & 0 & 0 & 0 & 0 & 0 & 0 & 0 & 0 & 0 & 0 & 0 & 0 & 0 & 0 & 0 & 0 \\
\hline Nitric Oxide & 0 & 0 & 0 & 0 & 0 & 0 & 0 & 0 & 0 & 0 & 0 & 0 & 0 & 0 & 0 & 0 & 0 & 0 & 0 & 0 & 0 & 0 & 0 & 0 \\
\hline Methanol & 0 & 0 & 0 & 0 & 0 & 0 & 0 & 0 & 0 & 0 & 0 & 0 & 0 & 0 & 0 & 0 & 0 & 0 & 0 & 0 & 0 & 0 & 0 & 0 \\
\hline Ethanol & 0 & 0 & 0 & 0 & 0 & 0 & 0 & 0 & 0 & 0 & 0 & 0 & 0 & 0 & 0 & 0 & 0 & 0 & 0 & 0 & 0 & 0 & 0 & 0 \\
\hline Propylene & 0 & 0 & 0 & 0 & 0 & 0 & 0 & 0 & 0 & 0 & 0 & 0 & 0 & 0 & 0 & 0 & 0 & 0 & 0 & 0 & 0 & 0 & 0 & 0 \\
\hline I-Butane & 0 & 0 & 0 & 0 & 0 & 0 & 0 & 0 & 0 & 0 & 0 & 0 & 0 & 0 & 0 & 0 & 0 & 0 & 0 & 0 & 0 & 0 & 0 & 0 \\
\hline N-Butane & 0 & 0 & 0 & 0 & 0 & 0 & 0 & 0 & 0 & 0 & 0 & 0 & 0 & 0 & 0 & 0 & 0 & 0 & 0 & 0 & 0 & 0 & 0 & 0 \\
\hline Cis-2-Butene & 0 & 0 & 0 & 0 & 0 & 0 & 0 & 0 & 0 & 0 & 0 & 0 & 0 & 0 & 0 & 0 & 0 & 0 & 0 & 0 & 0 & 0 & 0 & 0 \\
\hline I-Pentane & 0 & 0 & 0 & 0 & 0 & 0 & 0 & 0 & 0 & 0 & 0 & 0 & 0 & 0 & 0 & 0 & 0 & 0 & 0 & 0 & 0 & 0 & 0 & 0 \\
\hline N-Pentane & 0 & 0 & 0 & 0 & 0 & 0 & 0 & 0 & 0 & 0 & 0 & 0 & 0 & 0 & 0 & 0 & 0 & 0 & 0 & 0 & 0 & 0 & 0 & 0 \\
\hline Trans-2-Pentene & 0 & 0 & 0 & 0 & 0 & 0 & 0 & 0 & 0 & 0 & 0 & 0 & 0 & 0 & 0 & 0 & 0 & 0 & 0 & 0 & 0 & 0 & 0 & 0 \\
\hline Cyclopentane & 0 & 0 & 0 & 0 & 0 & 0 & 0 & 0 & 0 & 0 & 0 & 0 & 0 & 0 & 0 & 0 & 0 & 0 & 0 & 0 & 0 & 0 & 0 & 0 \\
\hline 2-2-DiMth-Butane & 0 & 0 & 0 & 0 & 0 & 0 & 0 & 0 & 0 & 0 & 0 & 0 & 0 & 0 & 0 & 0 & 0 & 0 & 0 & 0 & 0 & 0 & 0 & 0 \\
\hline 2-3-DiMth-Butane & 0 & 0 & 0 & 0 & 0 & 0 & 0 & 0 & 0 & 0 & 0 & 0 & 0 & 0 & 0 & 0 & 0 & 0 & 0 & 0 & 0 & 0 & 0 & 0 \\
\hline 2-Methylpentane & 0 & 0 & 0 & 0 & 0 & 0 & 0 & 0 & 0 & 0 & 0 & 0 & 0 & 0 & 0 & 0 & 0 & 0 & 0 & 0 & 0 & 0 & 0 & 0 \\
\hline 3-Methylpentane & 0 & 0 & 0 & 0 & 0 & 0 & 0 & 0 & 0 & 0 & 0 & 0 & 0 & 0 & 0 & 0 & 0 & 0 & 0 & 0 & 0 & 0 & 0 & 0 \\
\hline MthCyclopentane & 0 & 0 & 0 & 0 & 0 & 0 & 0 & 0 & 0 & 0 & 0 & 0 & 0 & 0 & 0 & 0 & 0 & 0 & 0 & 0 & 0 & 0 & 0 & 0 \\
\hline 2-4-DiMthPentane & 0 & 0 & 0 & 0 & 0 & 0 & 0 & 0 & 0 & 0 & 0 & 0 & 0 & 0 & 0 & 0 & 0 & 0 & 0 & 0 & 0 & 0 & 0 & 0 \\
\hline Cyclohexane & 0 & 0 & 0 & 0 & 0 & 0 & 0 & 0 & 0 & 0 & 0 & 0 & 0 & 0 & 0 & 0 & 0 & 0 & 0 & 0 & 0 & 0 & 0 & 0 \\
\hline 2-Methylhexane & 0 & 0 & 0 & 0 & 0 & 0 & 0 & 0 & 0 & 0 & 0 & 0 & 0 & 0 & 0 & 0 & 0 & 0 & 0 & 0 & 0 & 0 & 0 & 0 \\
\hline 3-Methylhexane & 0 & 0 & 0 & 0 & 0 & 0 & 0 & 0 & 0 & 0 & 0 & 0 & 0 & 0 & 0 & 0 & 0 & 0 & 0 & 0 & 0 & 0 & 0 & 0 \\
\hline 2-2-2-4riiMth-C & 0 & 0 & 0 & 0 & 0 & 0 & 0 & 0 & 0 & 0 & 0 & 0 & 0 & 0 & 0 & 0 & 0 & 0 & 0 & 0 & 0 & 0 & 0 & 0 \\
\hline MthCyclohexan & 0 & 0 & 0 & 0 & 0 & 0 & 0 & 0 & 0 & 0 & 0 & 0 & 0 & 0 & 0 & 0 & 0 & 0 & 0 & 0 & 0 & 0 & 0 & 0 \\
\hline 33-2Mth-1-Butene & 0 & 0 & 0 & 0 & 0 & 0 & 0 & 0 & 0 & 0 & 0 & 0 & 0 & 0 & 0 & 0 & 0 & 0 & 0 & 0 & 0 & 0 & 0 & 0 \\
\hline Dimethyl Ether & 0 & 0 & 0 & 0 & 0 & 0 & 0 & 0 & 0 & 0 & 0 & 0 & 0 & 0 & 0 & 0 & 0 & 0 & 0 & 0 & 0 & 0 & 0 & 0 \\
\hline Toluen & 0 & 0 & 0 & 0 & 0 & 0 & 0 & 0 & 0 & 0 & 0 & 0 & 0 & 0 & 0 & 0 & 0 & 0 & 0 & 0 & 0 & 0 & 0 & 0 \\
\hline o-xyle & 0 & 0 & 0 & 0 & 0 & 0 & 0 & 0 & 0 & 0 & 0 & 0 & 0 & 0 & 0 & 0 & 0 & 0 & 0 & 0 & 0 & 0 & 0 & 0 \\
\hline M-Xyle & 0 & 0 & 0 & 0 & 0 & 0 & 0 & 0 & 0 & 0 & 0 & 0 & 0 & 0 & 0 & 0 & 0 & 0 & 0 & 0 & 0 & 0 & 0 & 0 \\
\hline P-xylene & 0 & 0 & 0 & 0 & 0 & 0 & 0 & 0 & 0 & 0 & 0 & 0 & 0 & 0 & 0 & 0 & 0 & 0 & 0 & 0 & 0 & 0 & 0 & 0 \\
\hline $\mathrm{M}-\mathrm{Et}$ & 0 & 0 & 0 & 0 & 0 & 0 & 0 & 0 & 0 & 0 & 0 & 0 & 0 & 0 & 0 & 0 & 0 & 0 & 0 & 0 & 0 & 0 & 0 & 0 \\
\hline $1-2-4$ & 0 & 0 & 0 & 0 & 0 & 0 & 0 & 0 & 0 & 0 & 0 & 0 & 0 & 0 & 0 & 0 & 0 & 0 & 0 & 0 & 0 & 0 & 0 & 0 \\
\hline 2-Mth & 0 & 0 & 0 & 0 & 0 & 0 & 0 & 0 & 0 & 0 & 0 & 0 & 0 & 0 & 0 & 0 & 0 & 0 & 0 & 0 & 0 & 0 & 0 & 0 \\
\hline $1-2-4$ & 0 & 0 & 0 & 0 & 0 & 0 & 0 & 0 & 0 & 0 & 0 & 0 & 0 & 0 & 0 & 0 & 0 & 0 & 0 & 0 & 0 & 0 & 0 & 0 \\
\hline $23-21$ & 0 & 0 & 0 & 0 & 0 & 0 & 0 & 0 & 0 & 0 & 0 & 0 & 0 & 0 & 0 & 0 & 0 & 0 & 0 & 0 & 0 & 0 & 0 & 0 \\
\hline 2-Butene & 0 & 0 & 0 & 0 & 0 & 0 & 0 & 0 & 0 & 0 & 0 & 0 & 0 & 0 & 0 & 0 & 0 & 0 & 0 & 0 & 0 & 0 & 0 & 0 \\
\hline 3-Methylheptane & 0 & 0 & 0 & 0 & 0 & 0 & 0 & 0 & 0 & 0 & 0 & 0 & 0 & 0 & 0 & 0 & 0 & 0 & 0 & 0 & 0 & 0 & 0 & 0 \\
\hline 2-3-D & 0 & 0 & 0 & 0 & 0 & 0 & 0 & 0 & 0 & 0 & 0 & 0 & 0 & 0 & 0 & 0 & 0 & 0 & 0 & 0 & 0 & 0 & 0 & 0 \\
\hline 2-3-3-Trimth-C5 & 0 & 0 & 0 & 0 & 0 & 0 & 0 & 0 & 0 & 0 & 0 & 0 & 0 & 0 & 0 & 0 & 0 & 0 & 0 & 0 & 0 & 0 & 0 & \\
\hline 1-2-3-4-Tetramet & 0 & 0 & 0 & 0 & 0 & 0 & 0 & 0 & 0 & 0 & 0 & 0 & 0 & 0 & 0 & 0 & 0 & 0 & 0 & 0 & 0 & 0 & 0 & \\
\hline
\end{tabular}




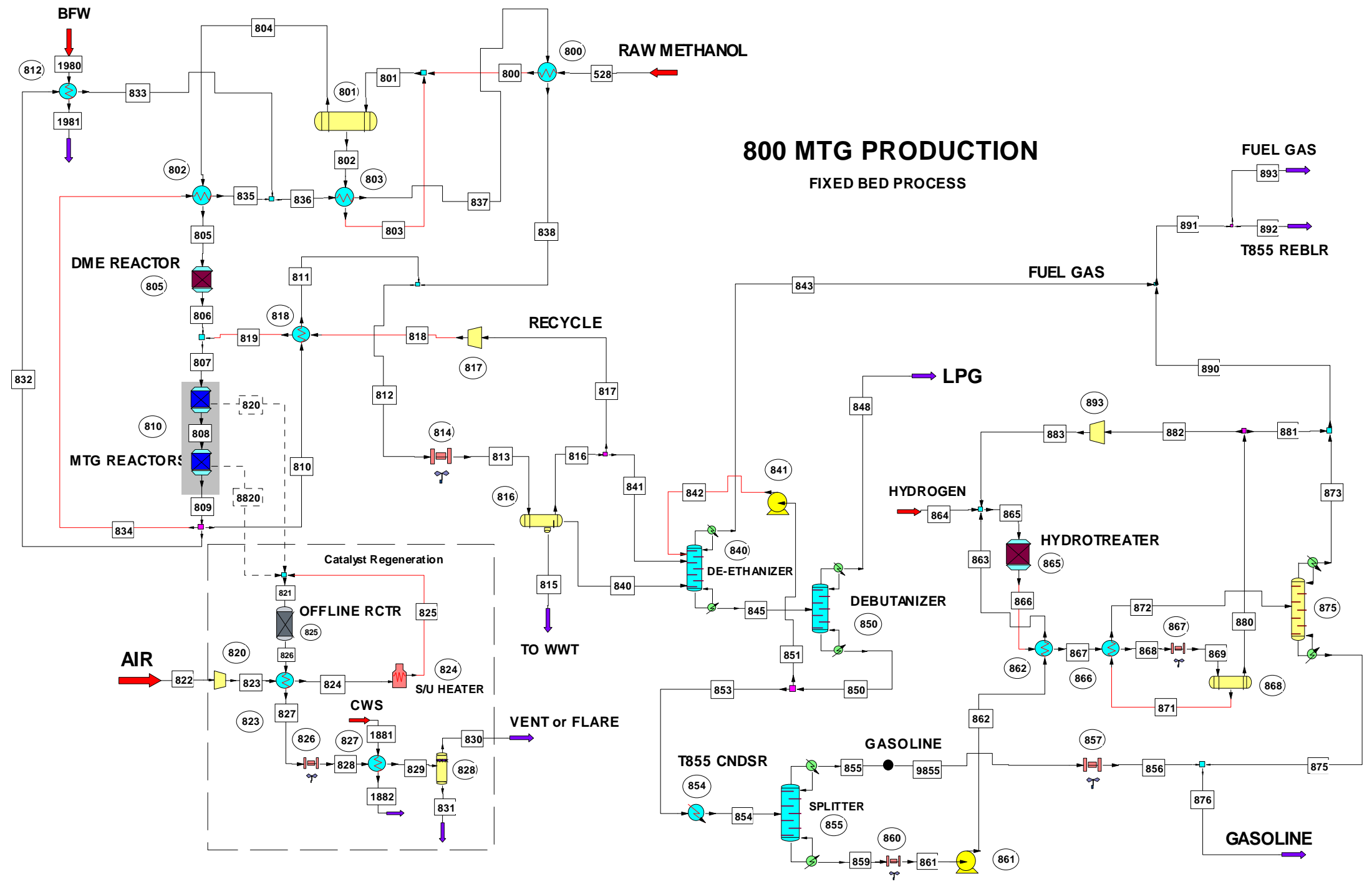

Figure B-2 Process Flows Diagram for the Directly-heated Gasifier Case - Gasoline Production 
Table B-2 Steam Results for the Directly-heated Gasifier Case - Gasoline Production

\begin{tabular}{|c|c|c|c|c|c|c|c|c|c|c|c|c|c|c|c|c|c|c|c|c|c|c|c|c|c|c|c|}
\hline Stream No. & 528 & 800 & 801 & 802 & 803 & 804 & 805 & 806 & 807 & 808 & 809 & 810 & 811 & 812 & 813 & 815 & 816 & 817 & 818 & 819 & 820 & 821 & 822 & 823 & & 824 & 825 \\
\hline $\begin{array}{l}\text { Temp F } \\
\text { Pres psia }\end{array}$ & $\begin{array}{r}112.175 \\
415\end{array}$ & $\begin{array}{l}200 \\
415\end{array}$ & $\begin{array}{r}354.081 \\
400\end{array}$ & $\begin{array}{r}354.081 \\
400\end{array}$ & $\begin{array}{l}358.097 \\
400\end{array}$ & 354.081 & 600 & $\begin{aligned} 757.725 \\
285\end{aligned}$ & 612.856 & 616.188 & $\begin{array}{r}707.248 \\
30\end{array}$ & 707.248 & $\begin{array}{r}198.771 \\
30\end{array}$ & 227.667 & 110 & 110 & 110 & 110 & & 600 & 5.188 & 574.528 & 70 & 102.76 & & 572 & 572 \\
\hline Enth MMBtu/h & $\begin{array}{r}415 \\
-311.75\end{array}$ & $\begin{array}{r}415 \\
-30581 \\
\end{array}$ & $\begin{array}{r}400 \\
-66744\end{array}$ & $\begin{array}{r}400 \\
-404.38\end{array}$ & $\begin{array}{r}400 \\
-36163\end{array}$ & $\begin{array}{r}400 \\
-263.06\end{array}$ & $\begin{array}{r}400 \\
55242\end{array}$ & $\begin{array}{r}385 \\
-25242\end{array}$ & $\begin{array}{r}385 \\
-28843\end{array}$ & $\begin{array}{r}330 \\
-28843\end{array}$ & $\begin{array}{r}300 \\
-28843\end{array}$ & $\begin{array}{r}300 \\
-18748\end{array}$ & $\begin{array}{r}300 \\
-2053.9\end{array}$ & $\begin{array}{r}300 \\
-31305\end{array}$ & 3200 & $\begin{array}{r}300 \\
33589\end{array}$ & $\begin{array}{r}300 \\
-2877\end{array}$ & $\begin{array}{r}300 \\
-2836\end{array}$ & 400 & $\begin{array}{r}400 \\
-26318\end{array}$ & $\begin{array}{r}330 \\
00268\end{array}$ & $\begin{array}{l}17 \\
224\end{array}$ & & $\begin{array}{r}17 \\
010334\end{array}$ & & $\begin{array}{r}17 \\
20792\end{array}$ & $\begin{array}{r}17 \\
20792\end{array}$ \\
\hline lapor mass fraction & 0 & & 0.41586 & & 0.98993 & & & & & $\begin{array}{l}2884.3 \\
1\end{array}$ & & & 0.96916 & 0.98663 & $\begin{array}{l}-3220.6 \\
0.91566\end{array}$ & & 827.1 & & 2810.8 & 2631.8 & 00268 & & & 334 & & 192 & \\
\hline tal lb/h & 95673.2 & & 230060 & & 134387 & & & & & & 114371 & & 724341 & 1114373 & 1114373 & 52452.3 & 35 & 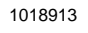 & 13 & 39 & 19.1199 & 17405 & .1 & & & 9.1 & \\
\hline $\begin{array}{l}\text { Wrates } \\
\text { thanol }\end{array}$ & 58.8 & 58.8 & 25193 & 5334 & 3534 & 658.4 & 58.4 & 65.84 & 165.8 & 0.006 & & & & 0.0002 & 1002 & & 000 & & & & 0 & & & & & & \\
\hline ater & 2.41 & 2.41 & 58.17 & 615.756 & .756 & 2.41 & 2.41 & & & 32291.1 & & & & 54922.2 & 54922.2 & & & & & & 0 & & & & & & 0 \\
\hline bon & & & 0 & $\begin{array}{r}0.15 .150 \\
0\end{array}$ & 0.100 & $\begin{aligned} 2.41 \\
0\end{aligned}$ & 0 & 0 & & Y.1.1 & 2.1 & $\begin{array}{r}9.4 \\
0\end{array}$ & $\begin{array}{r}9.4 \\
0\end{array}$ & (3492C.2 & $\begin{array}{r}34522.2 \\
0\end{array}$ & & & & $\begin{aligned} 4.01 \\
0\end{aligned}$ & & .1199 & & 0 & & $\begin{array}{l}0 \\
0\end{array}$ & 0 & \\
\hline rogen & 7.268 & 7.268 & 7.5149 & 0.2469 & 0.2469 & 7.268 & 7.268 & 7.268 & 5248.07 & 5248.68 & 5252.3 & 3414 & 3414 & 5252.19 & 5252.19 & 0 & 5250.41 & 5242.84 & 5242.84 & 5241.73 & & 0 & & & & 0 & 0 \\
\hline ide & & & 2616.52 & 234.162 & 234.162 & 382.36 & 32.36 & 2382.36 & 586437 & 586485 & 587168 & 381659 & 381659 & 587170 & 587170 & 0 & 584874 & 584030 & 584030 & 584054 & 0 & 0 & & & & & \\
\hline than & 3967 & 0.3967 & 83.2943 & 2.8977 & 2.8977 & 80.3966 & 80.3966 & 80.3966 & 163231 & 163269 & 163634 & 106362 & 106362 & 163634 & 163634 & 0 & 163385 & 163149 & 163149 & 163147 & 0 & 0 & & & & 0 & 0 \\
\hline iylen & 049 & 0.0049 & 0.0054 & 0.0004 & 0.0004 & 0.0049 & 0.0049 & 0.0049 & 3052.56 & 3054.47 & 3072.68 & 1997.24 & 1997.24 & 3072.69 & 3072.69 & 0 & 3056.71 & 3052.31 & 3052.31 & 3052.38 & 0 & 0 & 0 & & & 0 & 0 \\
\hline ane & 0109 & 0.0109 & 0.0118 & 0.0009 & 0.0009 & 0.0109 & 0.0109 & 0.0109 & 5790.97 & 5795.74 & 5841.29 & 3796.84 & 3796.84 & 5841.31 & 5841.31 & 0 & 5798.68 & 5790.32 & 5790.32 & 5790.55 & 0 & 0 & 0 & & & & \\
\hline poylene & & & & & & & & & 957.524 & 959.44 & 977.646 & 635.47 & 635.47 & 977.649 & 977.649 & 0 & 958.81 & 957.427 & 957.427 & 957.462 & 0 & 0 & 0 & & & 0 & \\
\hline ора & 0 & 0 & & & 0 & 0 & 0 & 0 & 79998 & 80179.5 & 81910.1 & 53241.6 & 53241.6 & 81910.4 & 81910.4 & 0 & & 79990 & 79990 & 8 & 0 & 0 & 0 & & & 0 & \\
\hline $\begin{array}{l}\text { utar } \\
\text { suta }\end{array}$ & $\begin{array}{l}0 \\
0\end{array}$ & $\begin{array}{l}0 \\
0\end{array}$ & $\begin{array}{l}0 \\
0\end{array}$ & $\begin{array}{l}0 \\
0\end{array}$ & $\begin{array}{l}0 \\
0\end{array}$ & $\begin{array}{l}0 \\
0 \\
0\end{array}$ & $\begin{array}{l}0 \\
0 \\
0\end{array}$ & $\begin{array}{l}0 \\
0 \\
0\end{array}$ & $\begin{array}{r}69344.5 \\
1767\end{array}$ & $\begin{array}{r}69679 \\
177097\end{array}$ & $\begin{array}{r}72867 \\
188837\end{array}$ & $\begin{array}{l}47363.5 \\
12274.4\end{array}$ & $\begin{array}{l}473363.5 \\
12274.4\end{array}$ & $\begin{array}{l}72867.1 \\
188383\end{array}$ & $\begin{array}{l}72867.1 \\
188838\end{array}$ & $\begin{array}{l}0 \\
0 \\
0\end{array}$ & $\begin{array}{l}69439.4 \\
17700.4\end{array}$ & $\begin{array}{l}69339.2 \\
176749\end{array}$ & 69339.2 & $\begin{array}{r}69341 \\
17752\end{array}$ & 0 & 0 & 0 & & 0 & 0 & 0 \\
\hline -2-Butene & 0 & 0 & 0 & 0 & 0 & 0 & $\begin{array}{l}0 \\
0 \\
0\end{array}$ & & 9542.76 & 9614.45 & $\begin{array}{l}18883.1 \\
10297.6\end{array}$ & $\begin{array}{l}12224.4 .4 \\
6693.41\end{array}$ & $\begin{array}{l}12224.44 \\
6693.41\end{array}$ & $\begin{array}{l}18828.8 \\
10297.6\end{array}$ & $\begin{array}{l}18883.8 \\
10297.6\end{array}$ & $\begin{array}{l}0 \\
0\end{array}$ & $\begin{array}{l}17700.4 \\
9550.01\end{array}$ & $\begin{array}{l}1 / 644.9 \\
9542.23\end{array}$ & $\begin{array}{l}17644.9 \\
9542.23\end{array}$ & $\begin{array}{l}1675.3 \\
9542.41\end{array}$ & 年 & 0 & & & & & 然 \\
\hline ent & 0 & 0 & 0 & 0 & 0 & 0 & 0 & & 27042.6 & 27425 & 31068.4 & 20194.4 & 20194.4 & 31068.4 & 31068.4 & & $\begin{array}{l}270001 \\
27081\end{array}$ & 27042 & 27042 & & 0 & 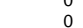 & & & & & \\
\hline en & 0 & 0 & 0 & 0 & 0 & 0 & 0 & & 5879.33 & 5984.46 & 198638 & 4541.15 & 4541.15 & 6986.49 & 698 & & & 587927 & 587927 & 587929 & 0 & & & & & & \\
\hline & & 0 & & & 0 & 0 & 0 & & 6494.62 & 6618.97 & 711.98 & 5012.78 & $\begin{array}{l}5012.78 \\
\end{array}$ & 7711.98 & & & & 6494.55 & 6494.55 & & 0 & & & & & & \\
\hline & 0 & 0 & 0 & 0 & 0 & 0 & 0 & & 97.347 & 716.467 & 98.645 & 84.119 & & 89 & & & & 697.355 & 55 & & 0 & & & & & & \\
\hline & 0 & 0 & & & & 0 & 0 & & 6354.32 & \$521.58 & 115.57 & 5275.12 & & 57 & & & & 6354.4 & 4.4 & & 0 & & & & & & \\
\hline & 0 & 0 & 0 & 0 & 0 & 0 & 0 & & 4866.02 & 5033.28 & 6627.27 & 4307.73 & 4307.73 & 27 & & & & 4866.14 & 4866.14 & & 0 & & & & & & \\
\hline & 0 & 0 & & & 0 & 0 & 0 & & 4556.68 & 4723.93 & 317.93 & 4106.65 & 4106.65 & .93 & & & & 4556.8 & 6.8 & 45 & & & & & & & \\
\hline & 0 & 0 & 0 & 0 & 0 & 0 & 0 & 0 & 4153.92 & 4321.18 & 5915.17 & 3844.86 & 3844.86 & 5915.17 & 591 & & 4160.05 & 4154.05 & 4154.05 & & 0 & & & & & & \\
\hline ine & 0 & 0 & 0 & 0 & 0 & 0 & 0 & & 1161.4 & 1256.99 & 1803.52 & 1172.29 & 1172.29 & & & 0 & 1163.12 & 1161.45 & 1161.45 & 116 & 0 & ) & & & & & \\
\hline Benze & 0 & 0 & 0 & 0 & 0 & 0 & 0 & & & 687 & & & & & & & & & & & 0 & 0 & & & & 0 & \\
\hline & 0 & 0 & 0 & 0 & 0 & 0 & 0 & & & & & & & & & & & & & & 0 & & & & & & \\
\hline ne & 0 & $\begin{array}{l}0 \\
0\end{array}$ & 0 & 0 & $\begin{array}{l}0 \\
0 \\
0\end{array}$ & 0 & 0 & & & & & & & & & & & & & & 0 & 0 & & & & & \\
\hline $\begin{array}{l}\text { ne } \\
\text { ne }\end{array}$ & $\begin{array}{l}0 \\
0\end{array}$ & $\begin{array}{l}0 \\
0\end{array}$ & $\begin{array}{l}0 \\
0\end{array}$ & $\begin{array}{l}0 \\
0\end{array}$ & $\begin{array}{l}0 \\
0 \\
0\end{array}$ & $\begin{array}{l}0 \\
0 \\
0\end{array}$ & $\begin{array}{l}0 \\
0 \\
0\end{array}$ & 0 & $\begin{array}{l}751.509 \\
709.079\end{array}$ & $\begin{array}{l}823.195 \\
780763\end{array}$ & $\begin{array}{r}1506.3 \\
1463.87\end{array}$ & $\begin{array}{l}979.096 \\
951.515\end{array}$ & $\begin{array}{l}979.0966 \\
9951515\end{array}$ & $\begin{array}{r}1506.3 \\
\end{array}$ & $\begin{array}{l}1506.3 \\
14638\end{array}$ & 0 & & 751.543 & 751.543 & & 0 & 0 & & & & 0 & 0 \\
\hline & 0 & 0 & & & 0 & 0 & 0 & & 571.545 & $\begin{array}{l}880.163 \\
643.231\end{array}$ & 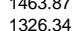 & $\begin{array}{l}951.515 \\
862.119\end{array}$ & $\begin{array}{l}951.515 \\
862119\end{array}$ & $\begin{array}{l}1463.87 \\
1326.34\end{array}$ & & & $\begin{array}{l}110.133 \\
572395\end{array}$ & $\begin{array}{r}09.109 \\
571.57\end{array}$ & $\begin{array}{r}709.109 \\
571.57\end{array}$ & 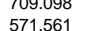 & 0 & & & & & & 0 \\
\hline & 0 & 0 & 0 & 0 & 0 & 0 & 0 & & & 570.593 & 1117.13 & 726.132 & & & & & 475 & & & & 0 & & & & & & 0 \\
\hline & 0 & 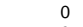 & & 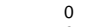 & 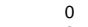 & 0 & 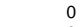 & & & & & 2893 & & & & & & & & & 0 & & & & & & \\
\hline & 0 & 0 & 0 & & 0 & 0 & 0 & & & & & & & & & & & & & & 0 & & & & & & \\
\hline & 0 & 0 & & 0 & 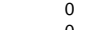 & 0 & r & & & & & & & & & & & & & & 0 & 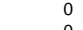 & 0 & & & 0 & \\
\hline & $\begin{array}{l}0 \\
0 \\
0\end{array}$ & 0 & & & 0 & $\begin{array}{l}0 \\
0 \\
0\end{array}$ & & & & & & & & & & & & & & & 0 & & & & & & 0 \\
\hline & 0 & $\begin{array}{l}0 \\
0\end{array}$ & $\begin{array}{l}0 \\
0\end{array}$ & 0 & $\begin{array}{l}0 \\
0\end{array}$ & 0 & 0 & 0 & $\begin{array}{l}185.306 \\
2382.68\end{array}$ & $\begin{array}{r}234.013 \\
2468.7\end{array}$ & $\begin{array}{l}689.458 \\
3197.36\end{array}$ & $\begin{array}{l}4288.147 \\
2078.28\end{array}$ & & $\begin{array}{l}68 \\
31\end{array}$ & & & & $\begin{array}{l}185.313 \\
23827\end{array}$ & 18 & & $\begin{array}{l}0 \\
0 \\
0\end{array}$ & o & & & & & $\begin{array}{l}0 \\
0 \\
0\end{array}$ \\
\hline & 0 & 0 & 0 & & 0 & 0 & 0 & & & & & & & & & & & & & & 0 & & & & & & \\
\hline & 0 & & 0 & & 0 & 0 & 0 & & & & & & & & & & & & & & 0 & & & & & & \\
\hline & 0 & & 0 & & 0 & 0 & 0 & & & & & & & & & & & & & & 0 & & & & & & \\
\hline & 0 & & & & 0 & 0 & 0 & 0 & & & 535. & 348 & & & & & & & & & 0 & 0 & & & 0 & & \\
\hline & 0 & 0 & & & $\begin{array}{l}0 \\
0\end{array}$ & $\begin{array}{l}0 \\
0 \\
0\end{array}$ & $\begin{array}{l}0 \\
0 \\
0\end{array}$ & $\begin{array}{r}0 \\
593026\end{array}$ & 1165 & $\begin{array}{l}1251.85 \\
509020\end{array}$ & 179 & 116 & & & & & & & & & 0 & & & & 0 & & \\
\hline & $\begin{array}{l}0 \\
0 \\
0\end{array}$ & 0 & & & $\begin{array}{l}0 \\
0\end{array}$ & $\begin{array}{l}0 \\
0 \\
0\end{array}$ & $\begin{array}{l}0 \\
0\end{array}$ & $\begin{array}{r}59302.6 \\
0\end{array}$ & 593 & $\begin{array}{r}59302.6 \\
234524\end{array}$ & $\begin{array}{r}0.0017 \\
14589\end{array}$ & $\begin{array}{r}0.0011 \\
048.931\end{array}$ & & & 0. & $\begin{array}{l}0 \\
0 \\
0\end{array}$ & & & & & 0 & 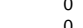 & & & & & 0 \\
\hline & 0 & 0 & 0 & 0 & 0 & 0 & 0 & & & & & $\begin{array}{l}948 . \\
291 .\end{array}$ & & & & & & 93.3 & 93. & & $\begin{array}{l}0 \\
0\end{array}$ & & & & & & \\
\hline - & 0 & 0 & & & 0 & 0 & 0 & & 5.2371 & 29.7007 & 266.022 & 172 & 172 & 266.022 & & 0 & & & & & & & & & & & \\
\hline uthNaphthalene & & 0 & & 0 & D & 0 & 0 & & 0.2521 & 4.2851 & 39.2736 & 25.5278 & 25.5278 & 39.2736 & 39.2736 & 0 & 0.2524 & 0.2521 & 0.2521 & 0.2521 & 0 & & & & & & \\
\hline ygen & & & & & & 0 & & & & & & & & & & 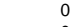 & & & & & 0 & & & & & & \\
\hline litrogen & & 0 & 1.9944 & 0 & 0.0567 & 0 & 0 & 0 & 1325.98 & 1325.98 & 1325.98 & $\begin{aligned} 861.886 \\
0\end{aligned}$ & $\begin{array}{c}861.886 \\
0\end{array}$ & 1326 & 1326 & & 0 & 0 & & 1323.72 & & & & & & & \\
\hline
\end{tabular}




\begin{tabular}{|c|c|c|c|c|c|c|c|c|c|c|c|c|c|c|c|c|c|c|c|c|c|c|c|c|c|c|}
\hline Stream No. & 826 & 827 & 828 & 829 & 830 & 831 & 832 & 833 & 834 & 835 & 836 & 837 & 838 & 840 & 841 & 842 & 843 & 845 & 848 & 850 & 851 & 853 & 854 & 855 & 856 & 859 \\
\hline Temp F & 1350.35 & 930.346 & 150 & 110 & 110 & 110 & 707.248 & 650 & 707.248 & 04.187 & 609.411 & 361.052 & 323.418 & 110 & 110 & 291.848 & 19.9982 & 262.052 & 8.613 & 290.641 & 290.641 & 290.641 & 96.713 & 66.717 & 149 & \\
\hline es psia & & & & & 7 & & 300 & 300 & 300 & & & 300 & 300 & 300 & 300 & & & 172 & & & & & & & & \\
\hline nth MMBtu/h & & 0.14654 & -3.2682 & -3.436 & -3.436 & 0 & -721.08 & -728.85 & -288.43 & -299.08 & -1027.9 & -1070.7 & -1076.6 & 36.992 & & 3.2539 & -14.076 & 26.834 & & & & & & & & \\
\hline $\begin{array}{l}\text { apor mass fraction } \\
\text { otal } \mathrm{lb} / \mathrm{h}\end{array}$ & $\begin{array}{r}1 \\
17005\end{array}$ & $\begin{array}{r}1 \\
005\end{array}$ & & $\begin{array}{l}1 \\
405\end{array}$ & $\begin{array}{l}1 \\
05\end{array}$ & $\begin{array}{l}0 \\
0 \\
0\end{array}$ & 279593 & $\begin{array}{r}1 \\
279593\end{array}$ & 111437 & 111440 & 300032 & $\frac{1}{30}$ & $\frac{1}{30003}$ & 0 & & 0 & 11 & 0 & & & & & & & & \\
\hline owrates in $1 \mathrm{~b} / \mathrm{h}$ & & & & & & & & & & & & & & & & & & & & & 26 & & 30933 & & & \\
\hline lethanol & 0 & 0 & 0 & 0 & 0 & 0 & 0.0001 & 0.0001 & & & 0.0001 & 0.0001 & 0.0001 & & & 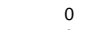 & 0 & 0 & 0 & 0 & 0 & 0 & 0 & 0 & 0 & \\
\hline ater & 0 & 0 & 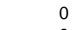 & 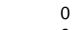 & 0 & & 13730.5 & 13730.5 & 5492.21 & 492.28 & 19222.8 & 19222.8 & 19222.8 & 21.8414 & 3.5301 & 0 & 25.3715 & 0 & 0 & 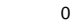 & 0 & 0 & 0 & & & \\
\hline bon & & & & 0 & 0 & 0 & & & & & & & & & & 0 & & 0 & 0 & 0 & & & & & & \\
\hline jioxide & & & & & & 0 & 1313.08 & 1313.08 & $\begin{array}{r}525.23 \\
587168\end{array}$ & $\begin{array}{l}525.117 \\
587174\end{array}$ & 1838.19 & $\begin{array}{l}1838.19 \\
00511\end{array}$ & 1838.19 & 1.7774 & $\begin{array}{r}7.5712 \\
8\end{array}$ & 0 & 9.3485 & 0 & 0 & 0 & 0 & 0 & 0 & 0 & 0 & \\
\hline $\begin{array}{l}\text { thon } \\
\text { thane }\end{array}$ & & $\begin{array}{l}.71 \\
0\end{array}$ & & $\begin{aligned} 71 \\
0\end{aligned}$ & $\begin{array}{r}71 \\
0\end{array}$ & & $\begin{array}{l}146792 \\
40084\end{array}$ & $\begin{array}{r}146792 \\
40084\end{array}$ & 58716.8 & 8719.4 & $\begin{array}{r}205511 \\
572716\end{array}$ & $\begin{array}{r}205511 \\
572716\end{array}$ & $\begin{array}{r}205511 \\
5772716\end{array}$ & $\begin{array}{l}2296.59 \\
248961\end{array}$ & $\begin{array}{l}843.397 \\
235603\end{array}$ & & $\begin{array}{l}3139.98 \\
484565\end{array}$ & $\begin{array}{l}0 \\
0 \\
0\end{array}$ & & & & $\begin{array}{l}0 \\
0 \\
0\end{array}$ & $\begin{array}{l}0 \\
0\end{array}$ & & & \\
\hline ne & 0 & 0 & 0 & 0 & 0 & 0 & $\begin{array}{r}40908.4 \\
768.17\end{array}$ & $\begin{array}{r}40908.4 \\
768.17\end{array}$ & $\begin{array}{l}16303.4 \\
307.268\end{array}$ & $\begin{array}{l}16303.11 \\
307.274\end{array}$ & $\begin{array}{l}1075.44 \\
1074.6\end{array}$ & $\begin{array}{l}5 / 211.6 \\
1075.44\end{array}$ & $\begin{array}{l}5 / 211.6 \\
1075.44\end{array}$ & $\begin{array}{l}248.961 \\
15.9708\end{array}$ & $\begin{array}{r}235.003 \\
4.4078\end{array}$ & 0 & 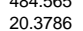 & 0 & & 0 & & 0 & 0 & & & \\
\hline lane & 0 & 0 & 0 & 0 & 0 & & 1460.32 & 1460.32 & 584.129 & 584.149 & 2044.47 & 2044.47 & 2044.47 & 42.6248 & 8.3618 & & 50.9866 & 0 & 0 & 0 & 0 & 0 & 0 & & 0 & \\
\hline pylene & 0 & 0 & 0 & 0 & 0 & 0 & 244.412 & 244.412 & 97.7646 & 97.7678 & 342.179 & 342.179 & 342.179 & 18.8394 & 1.3826 & & 11.4069 & 8151 & 3151 & & 0 & 0 & 0 & & 0 & \\
\hline par & 0 & 0 & 0 & 0 & 0 & 0 & 20477.5 & 20477.5 & 8191.01 & 8191.27 & 28668.8 & 28668.8 & & 1804.9 & 115.513 & & 536.619 & 1383.79 & 1383.8 & 0001 & & & & & & \\
\hline utar & 0 & 0 & $\begin{array}{l}0 \\
0 \\
0\end{array}$ & 0 & 0 & $\begin{array}{l}0 \\
0\end{array}$ & $\begin{array}{l}18216.7 \\
47203\end{array}$ & $\begin{array}{l}18216.7 \\
4720.7\end{array}$ & $\begin{array}{l}7286.69 \\
188037\end{array}$ & $\begin{array}{l}7286.87 \\
188941\end{array}$ & $\begin{array}{l}25503.6 \\
66020\end{array}$ & $\begin{array}{l}25503.6 \\
\end{array}$ & 25503.6 & 3427.72 & 100.133 & 5.8439 & $\begin{array}{l}86.8526 \\
82.851\end{array}$ & $\begin{array}{l}3446.84 \\
\end{array}$ & 3412.31 & $\begin{array}{r}34.5315 \\
0.96251\end{array}$ & 39 & 876 & 876 & 376 & 876 & \\
\hline-2. & & $\begin{array}{l}0 \\
0 \\
0\end{array}$ & & $\begin{array}{l}0 \\
0\end{array}$ & $\begin{array}{l}0 \\
0 \\
0\end{array}$ & $\begin{array}{l}0 \\
0 \\
0\end{array}$ & $\begin{array}{l}4720.93 \\
25739\end{array}$ & $\begin{array}{l}4720.93 \\
25739\end{array}$ & $\begin{array}{l}1888.37 \\
10276\end{array}$ & $\begin{array}{l}1888.41 \\
102977\end{array}$ & 6609.34 & 6609.34 & 6609.34 & $\begin{array}{l}1183.33 \\
70154\end{array}$ & $\begin{array}{l}25.5243 \\
\end{array}$ & 33.2291 & 28.8512 & $\begin{array}{l}1213.24 \\
7705790\end{array}$ & $\begin{array}{l}1016.89 \\
556,09\end{array}$ & 196.351 & 33.2291 & 163.122 & 163.122 & 163.122 & 63.122 & \\
\hline ent & 0 & $\begin{array}{l}0 \\
0\end{array}$ & $\begin{array}{l}0 \\
0 \\
0\end{array}$ & 0 & $\begin{array}{l}0 \\
0 \\
0\end{array}$ & $\begin{array}{l}0 \\
0 \\
0\end{array}$ & $\begin{array}{l}2574.39 \\
7767.09\end{array}$ & $\begin{array}{l}2577.39 \\
7767.09\end{array}$ & $\begin{array}{l}1029.76 \\
3106.84\end{array}$ & $\begin{array}{l}1029.977 \\
3106.87\end{array}$ & $\begin{array}{r}3600.416 \\
10874\end{array}$ & $\begin{array}{r}3600.416 \\
10874\end{array}$ & $\begin{array}{r}3600.16 \\
10874\end{array}$ & $\begin{array}{l}\begin{array}{l}741.564 \\
3987.39\end{array} \\
3\end{array}$ & $\begin{array}{l}133.7799 \\
39.0512\end{array}$ & $\begin{array}{r}34.344 \\
607.618\end{array}$ & $\begin{array}{l}20.10233 \\
46.3592\end{array}$ & $\begin{array}{l}7699.578 \\
4587.69\end{array}$ & $\begin{array}{l}5666.698 \\
997.274\end{array}$ & $\begin{array}{r}202.88 \\
3590.42\end{array}$ & $\begin{array}{r}34.334 \\
607618\end{array}$ & $\begin{array}{r}688.546 \\
29828\end{array}$ & $\begin{array}{r}168.546 \\
28828\end{array}$ & $\begin{array}{r}168.546 \\
29828\end{array}$ & $\begin{array}{l}6.98546 \\
20828\end{array}$ & \\
\hline en & 0 & 0 & 0 & 0 & 0 & 0 & 1746.6 & 1746.6 & 698.638 & 698.643 & 2445.24 & $\begin{array}{r}2445.24 \\
2445\end{array}$ & 2445.24 & 1098.62 & 8.4902 & 210.493 & $\begin{array}{r}\text { ob.552 } \\
8.3227\end{array}$ & 1309.28 & 65.4751 & 1243.81 & 210.493 & $\begin{array}{r}2020.0 \\
1033.32\end{array}$ & $\begin{array}{r}2020.0 \\
1033.32\end{array}$ & $\begin{array}{r}2902.0 \\
1033.31\end{array}$ & 1033.31 & \\
\hline ene & 0 & 0 & 0 & 0 & 0 & & 1927.99 & 1927.99 & 771.198 & 771.203 & 2699.2 & 2699.2 & 2699.2 & 1208.05 & 9.3788 & 222.351 & 9.3275 & 1430.45 & 116.572 & 1313.88 & 222.351 & 1091.53 & 1091.53 & 1091.53 & 1091.53 & \\
\hline & 0 & 0 & 0 & 0 & 0 & & 224 & 224.661 & 89.8645 & 89 & 314.526 & 314 & 314.526 & 20 & & & 0.5774 & .57 & & 28 & 75 & & & & 57 & \\
\hline & 0 & 0 & 0 & 0 & 0 & 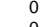 & 2028.89 & 2028.89 & 811.557 & 811.558 & 2840.45 & 2840.45 & 2840.45 & 1751.99 & 9.1764 & & 5.0396 & 13.8 & 434 & & 357.666 & & & & 1755.79 & \\
\hline & 0 & 0 & 0 & 0 & $\begin{array}{l}0 \\
0\end{array}$ & $\begin{array}{l}0 \\
0\end{array}$ & $\begin{array}{l}1656.82 \\
157948\end{array}$ & $\begin{array}{l}1656.82 \\
157948\end{array}$ & 662.727 & 66 & $\begin{array}{l}2319.54 \\
201107\end{array}$ & $\begin{array}{l}2319.54 \\
201107\end{array}$ & $\begin{array}{l}2319.54 \\
2210.17\end{array}$ & $\begin{array}{r}1754.1 \\
1751.5\end{array}$ & & 358.214 & 2.6404 & 16.7 & 0.0115 & & 14 & & $\begin{array}{l}1758.48 \\
175090\end{array}$ & & & \\
\hline e & $\begin{array}{l}0 \\
0 \\
0\end{array}$ & $\begin{array}{l}0 \\
0\end{array}$ & $\begin{array}{l}0 \\
0 \\
0\end{array}$ & $\begin{array}{l}0 \\
0\end{array}$ & $\begin{array}{l}0 \\
0 \\
0\end{array}$ & $\begin{array}{l}0 \\
0 \\
0\end{array}$ & $\begin{array}{l}1579.48 \\
1477979\end{array}$ & $\begin{array}{l}1579.48 \\
1477979\end{array}$ & $\begin{array}{l}\begin{array}{l}631.793 \\
591.1917\end{array} \\
5\end{array}$ & $\begin{array}{l}631.792 \\
591516\end{array}$ & 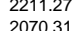 & $\begin{array}{l}2211.27 \\
2070.31\end{array}$ & $\begin{array}{l}2211.27 \\
20170.31\end{array}$ & $\begin{array}{l}1754.55 \\
175512\end{array}$ & $\begin{array}{l}6.5805 \\
5.9989\end{array}$ & $\begin{array}{r}358.303 \\
358.39\end{array}$ & $\begin{array}{l}2.2089 \\
1.7783\end{array}$ & $\begin{array}{l}2117.22 \\
21177.73\end{array}$ & $\begin{array}{l}0.0056 \\
0.0016\end{array}$ & $\begin{array}{l}2117.22 \\
2117.73\end{array}$ & $\begin{array}{r}358.303 \\
35833\end{array}$ & $\begin{array}{l}1758.91 \\
175924\end{array}$ & $\begin{array}{l}1758.91 \\
175924\end{array}$ & $\begin{array}{l}1758.91 \\
1759.34\end{array}$ & $\begin{array}{l}1758.91 \\
17524\end{array}$ & \\
\hline $\begin{array}{l}\text { tallat } \\
\text { ntane }\end{array}$ & 0 & 0 & 0 & 0 & 0 & & 450.88 & 450.88 & $\begin{array}{l}591.1517 \\
180.352\end{array}$ & $\begin{array}{l}591.516 \\
180.352\end{array}$ & $\begin{array}{l}2010.31 \\
631.232\end{array}$ & $\begin{array}{l}2010.311 \\
631.232\end{array}$ & $\begin{array}{l}2010.31 \\
631.232\end{array}$ & $\begin{array}{l}1 / 55.122 \\
640.394\end{array}$ & & $\begin{array}{r}388.39 \\
130.719\end{array}$ & $\begin{array}{l}1.1783 \\
0.3698\end{array}$ & $\begin{array}{l}2111.13 \\
772.421\end{array}$ & & $\begin{array}{l}2111.43 \\
772.421\end{array}$ & $\begin{array}{r}338.39 \\
130.719\end{array}$ & & $\begin{array}{l}1 / 59.934 \\
641.702\end{array}$ & & $\begin{array}{l}7199.34 \\
41.702\end{array}$ & \\
\hline Benzer & 0 & 0 & 0 & 0 & 0 & & 261.642 & $\begin{array}{l}461.600 \\
261.642\end{array}$ & 104.657 & $\begin{array}{l}104.657 \\
10.05\end{array}$ & 366.299 & 366.299 & 366.299 & $\begin{array}{l}399.217 \\
390.54\end{array}$ & 0.9335 & 81.4718 & 0.2037 & 481.418 & 0.0001 & 481.419 & 81.4718 & 399.947 & 399.947 & 399.946 & 46 & \\
\hline 2-4-Di & 0 & 0 & 0 & 0 & 0 & & & & 178 & & & & & 75 & & & 0.203 & & 0 & & 06 & & & & & \\
\hline & & 0 & 0 & 0 & 0 & 0 & & & & & & & & & & & & & 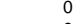 & & & & & & & \\
\hline & 0 & $\begin{array}{l}0 \\
0\end{array}$ & $\begin{array}{l}0 \\
0 \\
0\end{array}$ & 0 & 0 & 0 & & 376.576 & $\begin{array}{r}150.63 \\
\end{array}$ & & & $\begin{array}{l}527.206 \\
5120255\end{array}$ & & & & & 0.0963 & & 0 & & & & & & & \\
\hline & & $\begin{array}{l}0 \\
0 \\
0\end{array}$ & $\begin{array}{l}0 \\
0 \\
0\end{array}$ & $\begin{array}{l}0 \\
0\end{array}$ & $\begin{array}{l}0 \\
0 \\
0\end{array}$ & 0 & $\begin{array}{r}365.968 \\
331584\end{array}$ & $\begin{array}{r}365.968 \\
331584\end{array}$ & $\begin{array}{l}146.387 \\
{ }_{132} 2.34\end{array}$ & $\begin{array}{l}{ }_{146.387} \\
{ }_{132} 2.674\end{array}$ & $\begin{array}{l}512.355 \\
464.219\end{array}$ & $\begin{array}{l}512.355 \\
464.219\end{array}$ & 512.355 & $\begin{array}{l}753 \\
7753\end{array}$ & 1.024 & & 0.0848 & & 0 & & $\begin{array}{l}153.733 \\
15374\end{array}$ & & $\begin{array}{l}754.677 \\
75.47717\end{array}$ & 677 & 6.677 & \\
\hline & 0 & 0 & $\begin{array}{l}0 \\
0\end{array}$ & $\begin{array}{l}0 \\
0 \\
0\end{array}$ & 0 & & $\begin{array}{l}\begin{array}{l}3311.584 \\
279.282\end{array} \\
2\end{array}$ & 331.584 & $\begin{array}{l}\begin{array}{l}332.1644 \\
111713\end{array}\end{array}$ & $\begin{array}{l}\begin{array}{l}132.664 \\
111.713\end{array} \\
1\end{array}$ & $\begin{array}{l}\begin{array}{r}464.2199 \\
390.994\end{array} \\
3.96\end{array}$ & $\begin{array}{l}\begin{array}{r}664.219 \\
390.994\end{array} \\
3.96\end{array}$ & $\begin{array}{l}\begin{array}{r}664.219 \\
390.994\end{array} \\
3994\end{array}$ & $\begin{array}{r}53.943 \\
641.41\end{array}$ & $\begin{array}{l}0.8254 \\
0.686\end{array}$ & $\begin{array}{r}153 . / 41 \\
130.79\end{array}$ & $\begin{array}{l}0.0513 \\
0.0464\end{array}$ & 906 & 0 & $\begin{array}{l}58 \\
84\end{array}$ & $\begin{array}{r}153 . / 41 \\
130.79\end{array}$ & & & & 17 & \\
\hline ne & 0 & 0 & 0 & 0 & 0 & & 111 & 11 & 445.085 & 44 & 15 & 1557.8 & 7.8 & 809 & & & 4.1095 & 974 & 6.4406 & & & & & & & \\
\hline Tolue & 0 & 0 & 0 & 0 & 0 & & & & & & 141 & 1411.31 & 141 & & & & & & 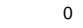 & & & & & & & \\
\hline & & & & 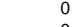 & 0 & & & & & & & & & & & & & & & & & & & & & \\
\hline & & 0 & 0 & 0 & 0 & & & 178 & & & 250.333 & $25 \mathrm{C}$ & & & & & & & 0 & & & & & & & \\
\hline & & $\begin{array}{l}0 \\
0 \\
0\end{array}$ & $\begin{array}{l}0 \\
0 \\
0\end{array}$ & $\begin{array}{l}0 \\
0\end{array}$ & 0 & c & $\begin{array}{l}172.364 \\
799339\end{array}$ & 172. & 68. & 68.5 & 241.311 & 241 & & & 76 & & 0.0064 & $\begin{array}{l}606 \\
098\end{array}$ & & 36 & & & & & & \\
\hline A-buene & & & 然 & $\begin{array}{l}0 \\
0 \\
0\end{array}$ & & & & $\begin{array}{r}99.3939 \\
31926\end{array}$ & & & $\begin{array}{l}1119 \\
446 .\end{array}$ & & & & & $\begin{array}{l}165.646 \\
211.423\end{array}$ & 1.438 & $9 / 8$ & $\begin{array}{r}0.02144 \\
0\end{array}$ & & $\begin{array}{l}165.646 \\
211.423\end{array}$ & & & & & \\
\hline & 0 & 0 & 0 & 0 & 0 & & & & & & & & & & & & & & & & & & & & & \\
\hline & 0 & 0 & 0 & 0 & 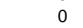 & & & & & & & & & & & & & & & & & & & & & \\
\hline & & 0 & 0 & 0 & & & & & & & & & & & & & & & & & & & & & & \\
\hline & & 0 & 0 & & & 0 & & & & & & & & & & & & & & & & & & & & \\
\hline Dimetl & & & 0 & 0 & 0 & 0 & 0.0 & 0.6 & & & & & & & & & & & & & & & & & & \\
\hline & 0 & & & 0 & $\begin{array}{l}0 \\
0\end{array}$ & & & & & & & & & & & & & & & & & & & & & \\
\hline & 0 & & 0 & & 0 & & & & & & & & & & & & & & & & & & & & & \\
\hline & 0 & & & 0 & 0 & & . 0.5 & 6.5054 & & & & & & & & 53.1 & & & & & & & & 5 & 05 & \\
\hline alene & & & & & & & 9.8184 & 9.8184 & 3.9274 & & 13.7458 & 58 & & 39.6 & 4 & 49 & & 46. & & 06 & 49 & 39 & 39 & & 0 & 0216 \\
\hline & & & & & & 0 & & & & & & & & & & 0 & & b & & & 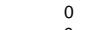 & & ) & & ) & \\
\hline & & & & & & & & & & & & & & & 1.9112 & & & & & & & & & & 0 & \\
\hline amet & & & & & & & & & & & & & & & & & & & & & & & & & & \\
\hline
\end{tabular}




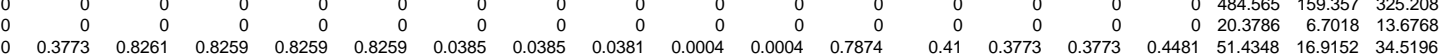




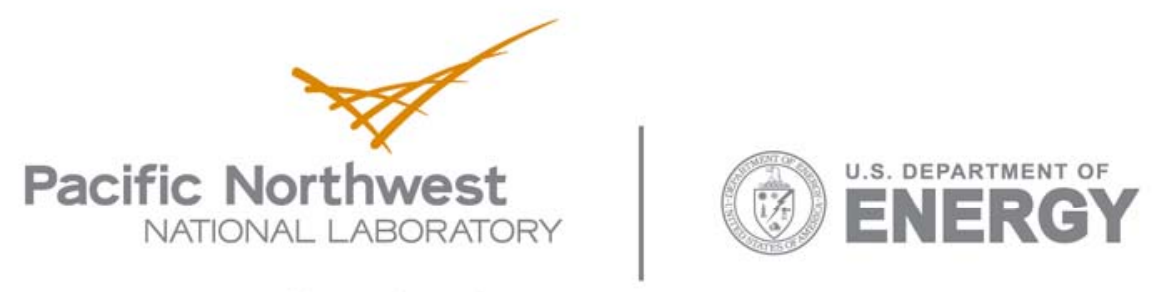

902 Battelle Boulevard

P.O. Box 999

Richland, WA 99352

1-888-375-PNNL (7665)

www.pnl.gov 